\title{
WHY DO PEOPLE PREPARE FOR NATURAL HAZARDS? AN APPLICATION OF THE THEORY OF PLANNED BEHAVIOUR TO HOUSEHOLD PREPARATION
}

\author{
BY \\ LAUREN JENNIFER VINNELL
}

\begin{abstract}
A thesis
submitted to the Victoria University of Wellington

in fulfilment of the requirements for the degree of

Doctor of Philosophy
\end{abstract}

Victoria University of Wellington

2020 


\section{Contents}

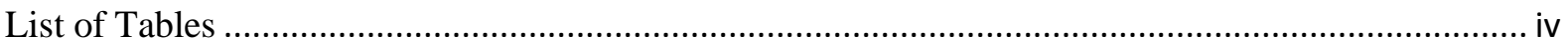

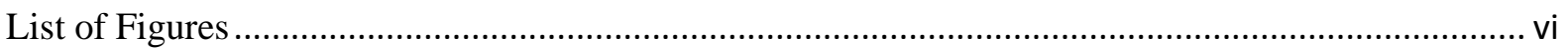

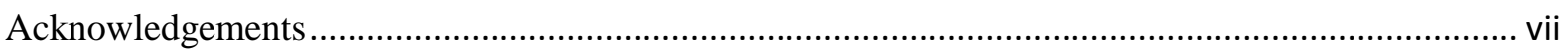

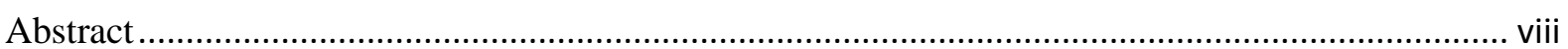

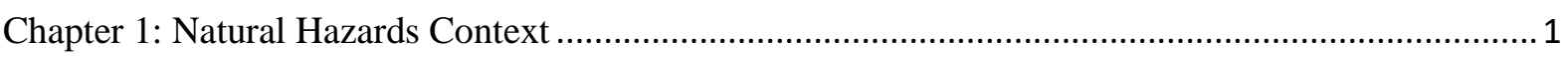

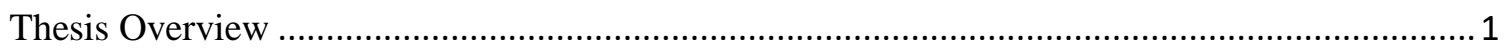

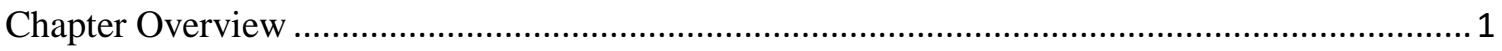

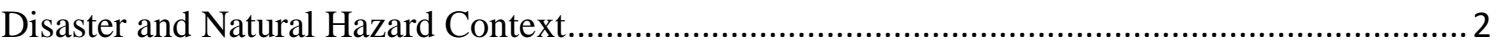

Interventions in the Natural Hazard Context .......................................................................... 10

Domain-Specific Definitions of Key Terms ........................................................................... 18

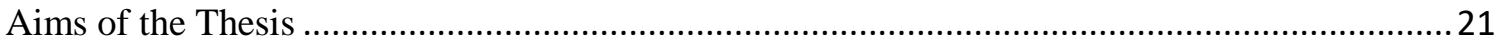

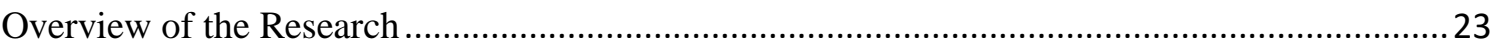

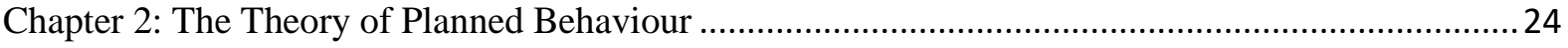

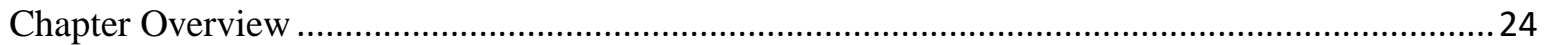

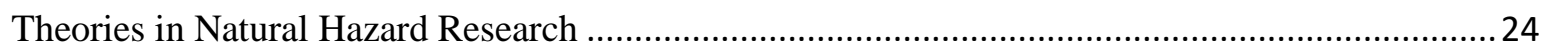

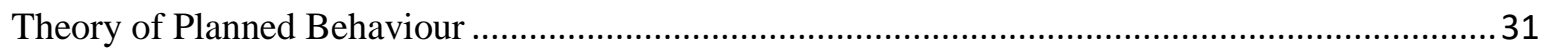

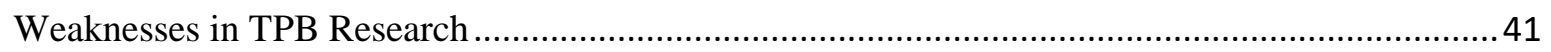

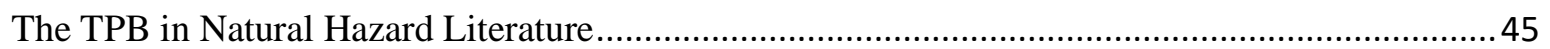

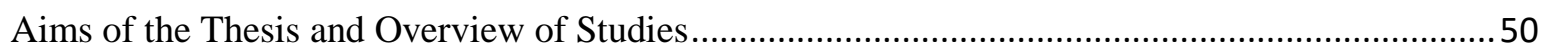

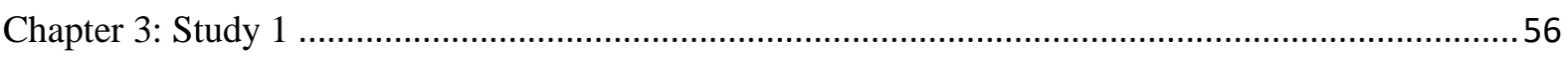

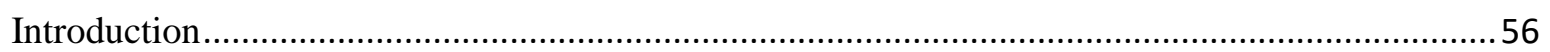

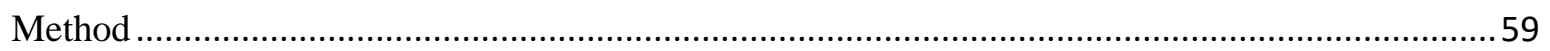

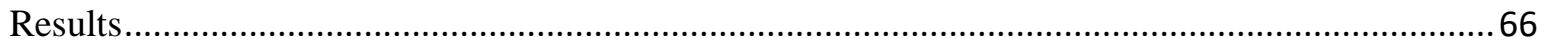

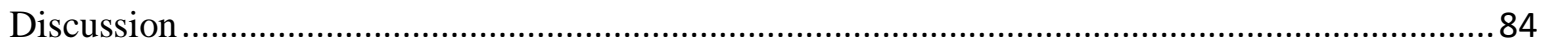

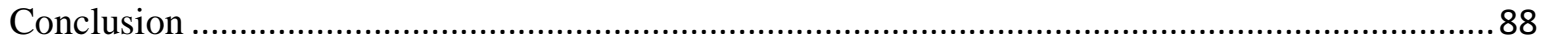

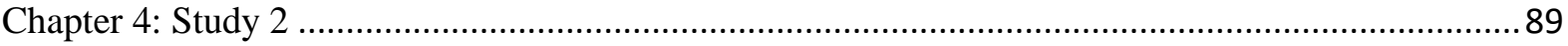

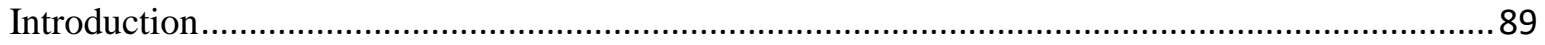

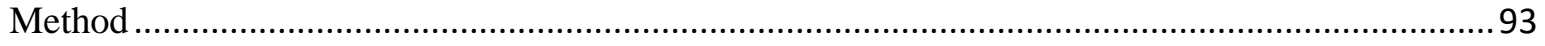

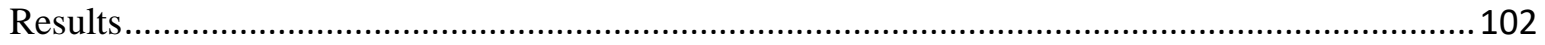

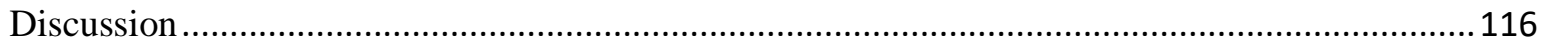

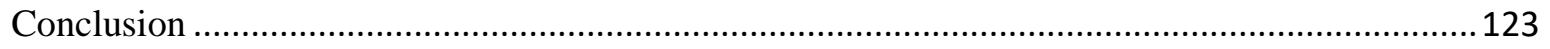

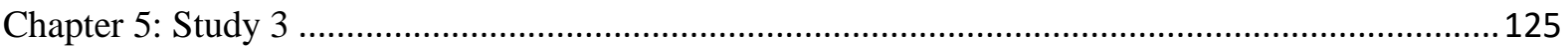

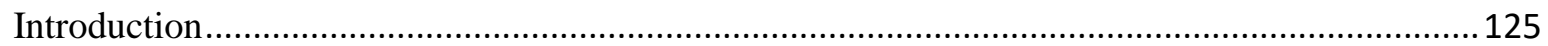




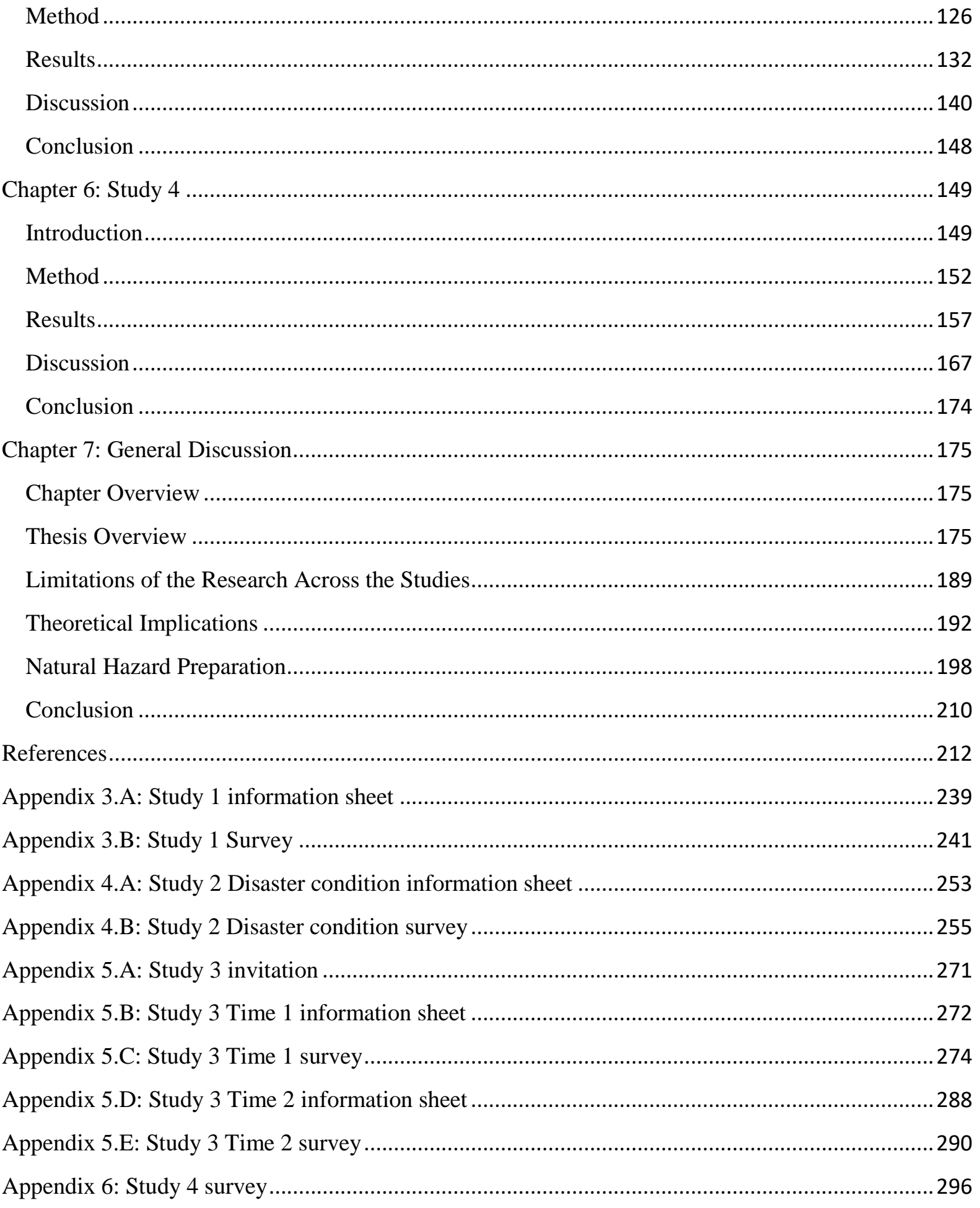


Table 2.1

Criteria used in theory selection

Table 3.1

Means and standard deviations of scale variables

Table 3.2

Frequency of preparation behaviours and mean scores of intentions, judgments of difficulty, and judgments of effectiveness

Table 3.3 .70

Rank order of preparation behaviours

Table 3.4

Frequency of indicated barriers to preparation behaviour

Table 3.5

Rank order of preparation behaviour barriers

Table 3.6 .75

Correlations between factors related to natural disaster preparation

Table 3.7 .78

Regression of subject and demographic variables on self-report general, survival, and mitigation natural disaster preparation

Table 3.8

Regression of subject and demographic variables on natural disaster preparation intentions

Table 3.9

Regression of TPB variables on self-report general, survival, and mitigation disaster preparation, controlling for demographic factors

Table 3.10

Regression of TPB variables on self-report natural disaster preparation intentions, controlling for demographic factors

Table 4.1

TPB component scale items for the Disaster condition

Table 4.2

Cronbach's alpha reliability scores for all TPB scales split by condition

Table 4.3

Descriptive statistics for all TPB scales, split by condition

Table 4.4

Results of Confirmatory Factor Analyses for individual TPB constructs within the Disaster condition

Table 4.5 108

Baseline model fit for the full TPB in the Disaster condition 
Table 4.6

Results of Confirmatory Factor Analyses for individual TPB constructs within the Hazard condition

Table 4.7

Baseline model fit for the full TPB in the Hazard condition

Table 5.1

Confirmatory factor analysis fit criteria results for individual TPB constructs

Table 5.2

Descriptive statistics for all TPB scales

Table 5.3

Correlations between intentions and TPB cognition components

Table 5.4

Fit criteria results for TPB models including and excluding two-factor belief distinctions

Table 6.1

Results of measurement invariance testing between the Treatment and Control conditions

Table 6.2 163

Differences in mean scores for TPB components between those who did ("Treatment") and those who did not ("Control") participate in the ShakeOut earthquake drill

Table 6.3

Results of structural equation modelling of associations between TPB components and intention for those who did ("Treatment") and those who did not ("Control") participate in the ShakeOut earthquake drill 


\section{List of Figures}

Figure 1.1. Map of the greater Wellington region of New Zealand's North Island.............................. 22

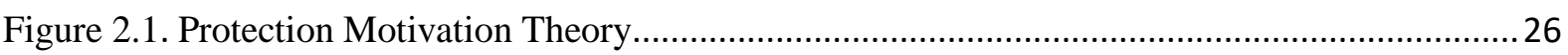

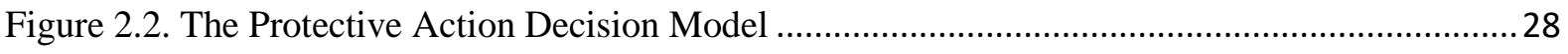

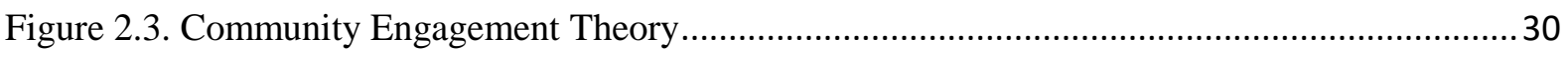

Figure 2.4. The original Theory of Planned Behaviour ................................................................ 34

Figure 2.5. The Theory of Planned Behaviour with the cognition components divided into their

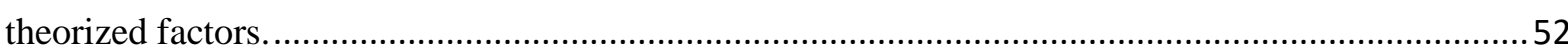

Figure 4.1. The Theory of Planned Behaviour with the cognition components divided into their

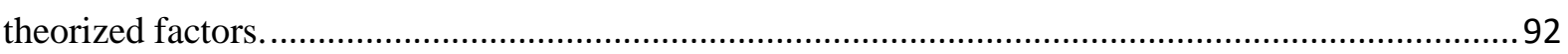

Figure 4.2. Structural equation model for the Disaster condition .................................................. 113

Figure 4.3. Structural equation model for the Hazard condition...................................................... 114

Figure 5.1. Structural equation model with attitude and PBC belief components not split...............135

Figure 5.2. Structural equation model with attitude and PBC belief components split....................137

Figure 7.1. The Theory of Planned Behaviour with the cognition components divided into their

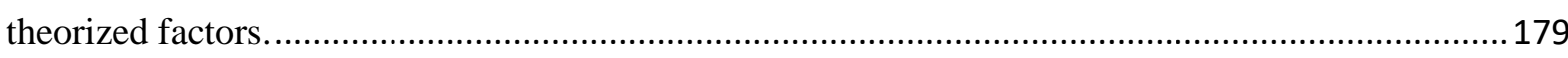

Figure 7.2. A poster as part of the National Emergency Management Agency's "Never Happens?

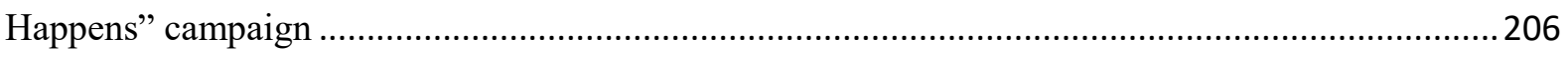




\section{Acknowledgements}

First, I want to thank my supervisors, Associate Professor Taciano Milfont and Professor John McClure, for their guidance, support, wisdom, and kindness during this process. This thesis would not be what it is without their tireless efforts both to help me with the work contained within and to help me grow as a researcher. I am grateful to have had the privilege of working with them, and the example they have set of conducting good research with passion is one which I will try to emulate in my own career. I would also like to thank the JCDR whānau, especially Julia and David, for their unwavering support. I feel very fortunate to have had the encouragement of such talented, hard-working, generous people.

Thanks must also go to my many study participants, without whom this research would not have been possible, and to the whole team at the School of Psychology, including the fantastic Admin and IT staff whose hard work keeps the wheels turning.

I was warned that doing a $\mathrm{PhD}$ would be an isolating experience. Thanks to the incredible students with whom I shared this journey, that warning could not have been farther from the truth. There are far too many to name, but I hope that they know that I am truly grateful for their support and friendship. A special shout-out has to go to the amazing Amanda, who has been a rock during the lows and the best hype-woman during the highs of this journey. It's been a privilege sharing this very special ride with you. To my incredibly patient non-uni friends who have put up with me talking about my research for the last three years, I appreciate all the encouragement you've given and the many times you've reminded me that there is life outside of study. I hope you know that this thesis wouldn't exist without you all either.

Finally, many thanks to my family, who have also put up with my rants (about both the stresses and the successes). In particular, my biggest thanks go to my wonder-woman mother who has been both the best role model and my most ardent supporter throughout my (long) university career. This is for you. 


\begin{abstract}
Natural hazards impact millions of people globally and lead to billions of dollars of economic loss each year. New Zealand is one of many countries vulnerable to multiple natural hazards including earthquakes, tsunami, volcanic eruption, and high impact weather. Despite these well-known risks, many residents of the capital city of Wellington have taken few steps to be more prepared. This location, therefore, presented an appropriate population for the investigations within this thesis. Decades of social science research has identified a multitude of factors related to preparation behaviour. However, many of these factors, such as risk perception and previous experience of natural hazards, are difficult to manipulate successfully in broad public education campaigns. The first main aim of this thesis was therefore to identify thoughts and beliefs about preparing which predict people's preparation behaviour, with the aim of identifying specific factors which can be efficiently and effectively targeted in campaigns encouraging preparation. This research was structured using the Theory of Planned Behaviour (TPB) which proposes a specific set of cognitions that affect intention formation as well as beliefs which inform those cognitions. In addition to using this model to structure the investigation, this thesis undertook several refinements and extensions to the model to address inconsistencies within TPB research. The intent of doing so was to provide a set of findings and a questionnaire which are not limited by those inconsistencies and which can be adapted for a range of behavioural contexts.

Four empirical studies were carried out, involving a total of 2,298 participants from the general population. Study 1 tested the applicability of this theory to the context of natural disaster preparation and clarified the nature of that context. Using an online survey of 722 residents of the urban Wellington region, Study 1 demonstrated low levels of preparation, identified not "getting around to it" as a main barrier to preparation, and supported a more extensive application of the TPB with the factors explaining approximately $16 \%$ of the variance in intentions. Study 2 tested a full TPB questionnaire including both the two-factor distinction (splitting attitudes into instrumental and experiential, norms in descriptive and injunctive, and perceived behaviour control into controllability and self-efficacy) and belief components which are proposed to precede attitudes, norms, and control. This study used an online sample of 603 Wellington residents. All the cognitions within the theory except perceived descriptive norms were significantly associated with either past behaviour or intentions to prepare, explaining approximately $47 \%$ of the variance in intentions. This study also included an experimental
\end{abstract}


framing manipulation, demonstrating benefits of referring to "natural hazard" preparation rather than "natural disaster" preparation.

Study 3 concluded the development of the TPB questionnaire by assessing intentions, cognitions, and beliefs at one point in time and behaviour one month later to allow for stronger inferences about causality, with a sample of 61 participants across both time points. This study used a different recruitment method than the previous studies: posted survey invites using addresses randomly selected from the electoral roll. Although this method did not produce a more demographically representative sample than the recruitment method used in Studies 1 and 2 as intended, Study 3 reproduced the findings of the "natural hazard" condition in Study 2. Finally, this study identified several key beliefs related to preparing such as the belief that preparing helps people to get through a natural hazard event better, that people can make the effort to prepare, and that preparing can be fun and rewarding. These beliefs offer specific and tangible factors which can be efficiently addressed in public education campaigns.

An intervention run previously by the New Zealand National Emergency Agency, the ShakeOut earthquake drill, was retrospectively evaluated in Study 4 by comparing those who did and those who did not participate $(N=480)$ using the TPB framework. Those who participated in the drill demonstrated better knowledge and use of the protective actions that are the focus of the drill than those who did not participate. Although this intervention was not informed by the findings of the previous studies, drill participants also demonstrated more positive scores for the TPB cognitions and intentions compared to non-participants, although more of the variance in intentions was explained for the latter (approximately 41\%) than the former (approximately 31\%).

Overall, findings from the empirical studies support the recommendation for consistent inclusion of all tested refinements to the Theory of Planned Behaviour (i.e., the two-factor distinction, the inclusion of belief measures, and careful development of behavioural measures). This thesis represents a uniquely thorough test of the Theory of Planned Behaviour to natural hazard preparation with important implications for both the contextual value of the theory and how the theory is applied in research broadly. The research also supports previous findings of the importance of instrumental attitudes and self-efficacy for natural hazard preparation and contributed the novel factor of experiential attitudes as well as identifying new, specific beliefs to target in public education campaigns. These contributions to our understanding of why people prepare for natural hazards can be used to encourage more people in Wellington, in New Zealand, and globally to be more prepared. 


\section{Chapter 1: Natural Hazards Context}

\section{Thesis Overview}

Globally, nationally, and locally, disasters severely impact human lives and economies (Wallemacq \& House, 2018). Social science has examined ways to reduce these impacts for decades, including extensive research internationally (Bourque, 2013; Lindell \& Perry, 2000; Paton, 2019; Solberg, Rossetto, \& Joffe, 2010), in New Zealand (Becker, Paton, \& Johnston, 2015), and Wellington specifically (Johnston et al., 2020). The goal of this thesis is to examine preparation for natural hazards using a leading behavioural theory to identify beliefs about preparing that can be efficiently targeted in public education campaigns (e.g., Paton \& Buergelt, 2019). This thesis examines the context of Wellington, but with the intention that the results are useful to practitioners throughout the rest of New Zealand and internationally.

\section{Chapter Overview}

As an introduction to this thesis, this chapter outlines the context for the research to be presented including a summary of the impacts of disasters and the challenges faced from natural hazards globally, nationally, and locally. Following this summary is an introduction of key terms related to the natural hazard context and the issues in the field around the definition and application of these terms. Finally, this chapter describes the overarching aim of this thesis and presents a brief outline of the studies included. Chapter 2 then reviews the central theory used in the presented studies.

The term "disaster" will be used when discussing actual, devastating events that might occur or have occurred with severe negative effects. The term "natural hazard" will be used when discussing risks from natural processes such as earthquakes ${ }^{1}$. While the survey in Study 1 used the term "natural disaster" in relation to preparation, the findings in Study 2 suggest that it is better to use the term "natural hazard". Therefore, this latter term will be used throughout, with the exception of Study 1 (Chapter 3). In discussions of specific pieces of research or reports, the term used within that work will be used here. That is, if a previous study used the term "disaster" then that term will be used in the review. Further, this thesis uses an all-hazards approach where participants are asked about preparing for natural hazards broadly rather than

\footnotetext{
${ }^{1}$ Other types of hazards, such as technological, anthropological, and biological, are beyond the scope of this research.
} 
a specific hazard. Some studies presented do focus on a particular hazard such as earthquakes. Where this is the case, that study will be discussed using the term applied in that research.

\section{Disaster and Natural Hazard Context}

Global disaster context. The United Nations Office for Disaster Risk Reduction created a summary document of economic and human losses due to disasters triggered by natural processes between 1998 and 2017 (Wallemacq \& House, 2018). During this 10-year period, disaster fatalities numbered 1.3 million, while another 4.4 billion people suffered injury, loss of home, displacement, or other outcomes which necessitated external help (Wallemacq \& House, 2018). Reported direct economic loss during this time amounted to US\$2.9 trillion, which does not include the $63 \%$ of disaster reports without data on economic impacts. An estimate by the World Bank puts global economic loss due to disasters triggered by natural processes at US $\$ 520$ billion per year (Wallemacq \& House, 2018). These impacts of disasters are worsening with climate change increasing the frequency and severity of meteorological events (Tippett, 2018) and population growth leading to more habitation of hazard-prone areas (Paton \& Buergelt, 2019), despite an exponential increase in academic publications regarding disaster risk reduction (Alexander et al., 2020).

In response to these increasing impacts and to help address the vast imbalance in funding assigned to recovery, the Hyogo Framework for Action 2005-2015 was developed. This framework laid out a plan to coordinate global efforts to reduce disaster impacts. New Zealand contributed, agreed to, and reported on progress on this framework as well as the subsequent Sendai Framework for Disaster Risk Reduction 2015-2030. The Sendai Framework has four main priorities: understanding risk, strengthening governance, investing in disaster risk reduction to increase resilience, and enhancing preparedness to respond to disasters. Part of the Sendai Framework agreement was for member nations to create national disaster resilience strategies; New Zealand's 10-year "National Disaster Resilience Strategy - Rautaki ā-Motu Manawaroa Aituā" was released in 2019 and was informed by the Sendai Framework, prioritizing risk management, improving response and recovery capability, and enabling and supporting community resilience (National Emergency Management Agency, 2019).

New Zealand's disaster and natural hazard context. New Zealand is prone to a range of natural hazards such as earthquakes, tsunamis, landslides, and floods (Khan, Crozier, \& Kennedy, 2012). This is due to a combination of multiple geographic factors such as New Zealand's location on the boundary of the Pacific and Australian tectonic plates and extensive, low-lying coast. Several sections of the plate boundary pose high risk to the country, including the Alpine Fault in the South Island, the Hikurangi subduction zone off the east coast of the 
North Island, and the Wellington fault (Johnston et al., 2013). In addition to the direct effects of earthquake shaking, earthquakes on these faults, in particular the offshore Hikurangi subduction zone, could trigger tsunami which would strike New Zealand coasts in minutes (Power, Wallace, \& Reyners, 2008). Earthquakes within the Asia-Pacific region, such as those that frequently occur throughout South East Asia, and global earthquakes, typically off the west coast of South America, could also trigger tsunami which threaten New Zealand (Horspool, Cousins, \& Power, 2015). Finally, several regions of New Zealand, namely the central plateau of the North Island and Auckland, the country's largest city, are prone to volcanic activity (Magill \& Blong, 2005). As well as direct effects of eruptions, including ground shaking, lava flow, and lahars, ashfall could negatively impact the country's important agriculture industry (Wilson \& Cole, 2007).

In addition to these geophysical hazards, New Zealand is prone to severe meteorological hazards, such as ex-tropical cyclones which can cause significant damage to infrastructure not designed to withstand such events (Daly, 2018). Droughts, floods, snowstorms, and wildfires also pose a risk to populations and industries in New Zealand (Ministry for Primary Industries, 2014). These types of hazards differ in terms of the risk they pose; sudden shocks such as earthquakes are more difficult to forecast and have much longer return periods (i.e., occur less frequently) but typically cause significantly more damage than "seasonal" events such as fires and floods which occur more frequently (United Nations General Assembly, 2016).

Several natural hazard events have occurred throughout New Zealand's brief history ${ }^{2}$, such as the 1855 Wairarapa earthquake which drastically changed the coastline around Wellington (Johnston et al., 2013) and the 1931 Napier earthquake which triggered one of the first disasters in New Zealand, causing extensive injuries and fatalities (Davison, 1934). The latter half of the $20^{\text {th }}$ century was relatively quiescent in terms of natural hazard events. However, recent events such as the 2010/11 Canterbury and 2016 Kaikōura earthquake sequence, the 2017 flooding in Edgecumbe caused by Cyclone Cook, the 2019 Nelson fires, and the 2019 Whakaari/White Island eruption have reinforced the risk posed by natural hazards. The February $22^{\text {nd }}, 2011$, Christchurch earthquake alone claimed 185 lives, with the full earthquake sequence causing an estimated NZ $\$ 40$ billion dollars of loss (Potter, Becker, Johnston, \& Rossiter, 2015). This sequence, and in particular the February 2011 aftershock,

\footnotetext{
${ }^{2}$ The following events occurred since British settlement of New Zealand; other events are recorded in Indigenous Māori kōrero nehe (history) which are not discussed here.
} 
led to extensive and ongoing debate around how to reduce the impacts of such events in future, including changes to building codes (McRae, McClure, Henrich, Leah, \& Charleson, 2017).

More recently, the 2016 Kaikoura earthquake claimed two lives and triggered over NZ\$1 billion dollars of loss (Stevenson et al., 2017). This earthquake and the resulting tsunami warning served as a reminder that Wellington is likely to suffer severe negative impacts after a rupture on a local fault and that many suburbs in the area are at risk. The last significant rupture in this region was the aforementioned 1855 Wairarapa earthquake and ruptures on the local faults causing fatalities are expected approximately every 120 years (Smith, 2015).

However, awareness of a risk does not necessarily lead to preparation. For example, the risk of earthquakes in Wellington is well known and yet many citizens are largely unprepared for such events (Johnston et al., 2013; Khan et al., 2012). The New Zealand government has invested millions of dollars in improving infrastructure, passed legislation to increase the resilience of building stock, and funded promotional campaigns to encourage individuals to prepare (e.g., Kaye, 2016). However, evidence from previous research suggests that these education programmes have not led to significant increases in preparedness (Johnston et al., 2013; Lindell \& Whitney, 2000; Paton, Smith, \& Johnston, 2005), perhaps because there is a disconnect in the beliefs about hazards between the public and those who are communicating risk information, rather than that information not being of a high enough quality (Paton \& Buergelt, 2019). It is therefore important to understand the beliefs held by citizens which affect their preparation behaviour. New Zealand has the potential to experience an event more damaging than any in recent history, at the level of the 2004 Indian Ocean Tsunami or 2011 Tohuku earthquake and tsunami, such as a megathrust earthquake on the Hikurangi subduction zone (Crawford, Saunders, Doyle, Leonard, \& Johnston, 2019). Although a large amount of research has aimed to understand the impacts of both the Canterbury earthquake sequence and the Kaikōura earthquake (Vinnell, Orchiston, Becker, \& Johnston, 2019), the country is likely not yet prepared to fully handle a worst-case event. For example, should an earthquake the magnitude of the 2011 Tohuku event strike Wellington, it is possible that the city would never recover (George, 2018).

Wellington's disaster history. While New Zealand is relatively uniformly exposed to natural hazard risk, the types of hazard which pose the largest risk do vary regionally. Most fatal natural hazard events in Wellington have either been seismic or meteorological. Earthquake events which led to the loss of life include the 1855 Wairarapa earthquake and tsunami, the later 1942 Wairarapa earthquake, and the 2016 Kaikōura earthquake; although neither of the fatalities resulting from the latter event occurred in Wellington, significant 
impacts were felt in the city and region. The other natural hazard events which have caused deaths in Wellington were storm-related; flooding in the Hutt Valley killed 14 in 1858 and one person in 2015, while Tropical Cyclone Giselle led to the sinking of the TEV Wahine in 1968, leading to 53 deaths.

The sudden, infrequent nature of earthquakes compared to the more predictable, seasonal nature of storms means that the events experienced in Wellington cover one key dimension on which hazards differ. However, it is important to acknowledge that beliefs about earthquake preparedness in Wellington might differ from, for example, beliefs about preparing for volcanic eruptions in Auckland, where the objective risk is similar but there have been no disaster events in the region since colonization. Beliefs about both of these risks might also differ from beliefs about preparing for drought in the heavily agricultural areas of the South Island which tends to have a lower impact but occurs more frequently. It is therefore important to acknowledge the specific hazard context of the geographical area under study.

Different types of natural hazard preparation. Hazard preparation relates to actions taken to ensure a better outcome following a natural hazard event. These actions can aim to reduce the number of people exposed to natural hazards, such as by restricting construction in areas prone to natural processes (e.g., floodplains, coastlines) or to reduce the impact of natural hazards, such as by improving the ability of building stock to withstand earthquake shaking. Preparation can be undertaken by individuals and various levels of government, directly or indirectly. For example, individuals can prepare at the household level by storing emergency supplies, making response plans with family, and checking the strength of their home. Governments can prepare through direct actions such as investing in improvements to public infrastructure (e.g., the Wellington Lifelines Resilience Project; Brown et al., 2019) and through indirect actions such as passing legislation requiring individual action (e.g., New Zealand's recent legislation to strengthen earthquake-prone buildings; McRae et al., 2017). Some research has explored the impact of perceptions of government versus personal responsibility (e.g., Becker et al., 2015; Paton, Smith, \& Johnston, 2000). Research in the United States demonstrates that most people view individuals as responsible for preparing themselves (Arlikatti, Lindell, \& Prater, 2007) but that some types of preparation, such as strengthening public buildings, should be the responsibility of local government (Flynn, Slovic, Mertz, \& Carlisle, 1999). For the best possible outcome in a natural hazard event, all the above types of preparation should be implemented.

In Wellington, the context of this research, the council is investing millions of dollars to improve the resilience of key lifeline infrastructure including roads, water, and electricity 
(Wellington City Council, 2017). Such effort reflects the recent global shift towards resilience investment at the level of infrastructure (e.g., the 100 Resilient Cities project). One economic assessment estimated that every US\$1 investment from government in resilience reduces economic loss from disasters by up to US\$4 (Hallegatte, Rentschler, \& Rozenberg, 2019). However, there is a perception among citizens that the government will also provide supplies such as food in a disaster (Shadwell, 2016). The Wellington Region Emergency Management Office recently changed their stocked Civil Defence shelters to Community Emergency Hubs which serve as gathering points for communities to coordinate their response but are not stocked with survival items (Shadwell, 2016). Therefore, in Wellington as in the rest of New Zealand and many hazard-prone areas globally, regardless of ongoing government work, it is important for individuals to take steps to ensure they both survive initial impacts of natural hazards and can look after themselves in the following period of disruption (Paton \& Buergelt, 2019). Increasing individual preparedness, which remains low globally, will reduce loss and help speed recovery following natural hazard events (Paton \& Buergelt, 2019).

Household-level preparation actions, which are the focus of this research, can be grouped into survival actions to improve chances of survival after the event, such as having food and water stored, and mitigation actions to reduce the risk and/or damage from the event, such as strengthening buildings (Russell, Goltz, \& Bourque, 1995; Spittal, McClure, Siegert, $\&$ Walkey, 2008). Survival actions tend to be carried out more frequently than mitigation actions (McClure, Spittal, Fischer, \& Charleson, 2015). While local and national government is working to improve Wellington and New Zealand's resilience to natural hazards as part of various strategies (e.g., Wellington City Council's resilience strategy as part of the international 100 Resilient Cities project), only a minority of people are "fully" prepared at the individual level (13\%; Colmar Brunton, 2018; the word "fully" is in quotations here because the definition of being fully prepared in the referenced survey only requires having completed four survival actions); this leaves a large proportion of the population who would benefit from empiricallysupported behaviour change interventions.

Along with knowledge of a hazard and its potential impacts, survival and mitigation actions are considered "traditional" types of preparation (Nojang \& Jensen, 2020). Other types of preparedness are increasingly being considered within the literature, such as psychological preparedness (e.g., McLennan, Marques, \& Every, 2020). This type of preparedness includes factors such as knowledge, anticipation, and awareness, as well as beliefs about ability to manage impacts. This type of preparedness overlaps with the "mental and physical adaptive capacity" component of preparedness in the Holistic Individual Preparedness Model (HIPM; 
Nojang \& Jensen, 2020), which refers to the capacity to cope without support, considering the role of factors such as pre-existing mental health issues. This model also considers technological integration, which emphasises the importance of access to technology to receive warning, response, and recovery information, and social integration, which emphasises the role of social networks in informing behaviour as well as providing support. The increasing awareness of the importance of this latter type of preparedness is reflected in recent shifts in messaging in New Zealand, such as the Wellington Region Emergency Management Office encouraging people to meet their neighbours so that they are better prepared to support each other in a natural hazard event. Unfortunately, including this type of preparedness is beyond the scope of this thesis but presents a potential avenue for future research.

The proposed research in this thesis seeks to examine why people prepare themselves for natural hazards and identify social psychological concepts which can be efficiently targeted in broad public education campaigns to increase individual preparation behaviours. Globally and nationally, the combined efforts to increase mitigation actions, survival actions, and response and recovery capabilities at all levels (e.g., individual, community, city, and country) has been represented as an effort to increase resilience to natural hazards. Given the broad reach of the concept of resilience and the diverse research areas which contribute to achieving preparedness goals, a brief review of relevant literature is presented below.

The concept of resilience. Several authors of disaster literature have discussed the definition of resilience specific to this field (Kelman, 2018). Some researchers argue that it is not necessary, not beneficial, and even not possible to precisely define or agree on a definition of "resilience" (Elms, 2015; Kelman, 2018). The priority is to ensure clarity as to how research fits into the broad concept and context (Hobfoll, Stevens, \& Zalta, 2015). However, most definitions of resilience share core aspects. Put simply, resilience refers to withstanding or reducing negative effects of natural hazards on most areas of life (Cooper-Cabell, 2013). Paton, Millar, and Johnston (2001) defined resilience as the ability to bounce back after a hazard event. The more resources a community has, the less difficult it is to cope through and after a disaster. Some definitions of resilience have different nuances, such as that given by Hobfoll et al. (2015) construing the human element of resilience as "the ability of people or their social systems...to withstand the impact of major or traumatic stress, meaning that they remain functional or unharmed on some deep lasting level" (p. 174).

Paton et al. (2001) identified three components of resilience: dispositional (personal characteristics; e.g., self-efficacy), cognitive (e.g., coping style), and environmental (e.g., sense of community). These components relate to definitions of resilience at different levels. For 
example, dispositional aspects influence individual resilience, or the ability of a person or small group such as a family to cope and recover. The environmental components, such as sense of community, relate to community resilience. For example, the resilience of a community includes how well it can respond and recover in a disaster (Elms, 2015) as well as adapt to long-term outcomes (Cooper-Cabell, 2013; Vallance, 2015). These different definitions, components, and levels of resilience mean that the concept is not measured consistently across studies.

One key challenge to consistent measurement of community resilience in particular is that such a measure requires not only an operationalization of resilience, but an operationalization of community. Communities themselves are hard to define and delineate (Elms, 2015), especially when treated as socially constructed; that is, individuals can selfidentify as belonging to a community based on their geographical location or membership in particular social or cultural groups, which leads to overlap in definitions but differences in real, lived experiences of community (MacQueen et al., 2001). Paton et al. (2001) argued that the factors which predict or comprise resilience, in whatever definition, should translate and be useful across groups and situations. Given the different forms a community may take, finding a measurement tool as well as intervention strategies which can be used beyond the population with which they were developed presents a significant challenge. An important consideration with these strategies is that, although resilience can be defined at several levels, one person's resilience can benefit others. For example, current messaging from groups such as New Zealand's National Emergency Management Agency encourages people to identify ways that they may be able to help their neighbours which will be easier if they are able to look after themselves. Therefore, research could target individual resilience, avoiding the complexities of examining communities, based on the assumption that more resilient individuals will lead to more resilient communities. This is the perspective adopted in this thesis.

When considering disaster risk, researchers often distinguish four key aspects: reduction, readiness, response, and recovery. The former two aspects are when resilience can be developed, while the latter two are when resilience can be demonstrated (Mamula-Seadon $\&$ McLean, 2015). Response refers to "immediate actions to save lives, protect property and the environment, and meet basic human needs in emergency situations" (Mamula-Seadon \& McLean, 2015, p. 84-85). Response therefore is easier if individuals can be to a degree selfsustaining. For example, having stores of food at home acts as a buffer so that people can support themselves during disruption to supply chains (Elms, 2015). In cities such as Wellington, local response capabilities are likely to be exceeded in a major earthquake so there 
is a push from government for individuals to be self-sufficient (Mamula-Seadon \& McLean, 2015).

Recovery can be defined as the process to restore all types of environment, including built, social, psychological, economic, and natural, which should involve providing support to communities affected by disasters (Mamula-Seadon \& McLean, 2015). Recovery in Christchurch following the September 2010 and February 2011 earthquakes started as soon as two days after the earthquake, with much of the immediate response work already completed. This was largely due to the city's location on a plain which meant that 27 access routes remained useable after the earthquake (B. Pepperell, personal communication, March 7, 2019). External supplies and support could reach the city immediately and in large quantities, so with needs such as food, water, and medical assistance quickly met, the city could begin the long (and still-continuing process) of recovery. The social impacts of the Canterbury earthquakes stemmed from problems such as poor living conditions and struggling businesses which have led to long-term stress and psychological problems including depression and anxiety (CooperCabell, 2013).

The Canterbury earthquake sequence produced valuable lessons for those dealing with improving disaster response and recovery. However, most of the difficulty in Christchurch was in recovering long-term rather than coping with the immediate impact (Elms, 2015). Different cities, both in New Zealand and internationally, are likely to encounter different challenges following a large earthquake event. It is therefore useful to consider a case study of an event in New Zealand which will likely look notably different from the Christchurch event.

For example, aside from the frequently discussed issues with Wellington's building stock (e.g., Sachdeva, 2016), because of the city's geography a large earthquake could split the wider region into seven "islands": areas within the city and wider region which would be so thoroughly cut off that they would effectively be unreachable islands (George, 2017). These seven "islands" would be comprised of 23 "road islands": 23 different areas of the region with no roading access which would take up to 120 days to fully reconnect (Brown et al., 2019). Both State Highways out of Wellington would likely be unusable following a large earthquake (Kos, 2010). Limited access to the airport would mean that the only viable route for getting resources into the city would be through the port at the rate of approximately 400 containers a day. However, damage to the port following the November 2016 Kaikōura earthquake showed that even this route of access is vulnerable. One estimate gives one to three months as a timeframe for the port to resume even minimal operation (Brown et al., 2019). On top of this, some calculations have as many as 12,000 people suffering injuries, which would likely exceed 
the capabilities of the isolated health services (George, 2017), and that electricity will be disrupted for up to six months and water for up to 12 months (Brown et al., 2019).

While the situation described above establishes the importance of Wellington for a case study for increasing individual resilience to natural hazards, it is intended that the research within this thesis will be relevant and useful both throughout New Zealand as well as in other countries facing similar challenges. There are several important considerations when generalizing the findings of this thesis to other contexts, in New Zealand and internationally, in particular relating to intra- and international cultural differences

\section{Culture.}

International comparisons. It is typical to draw on literature from different countries when developing studies and hypotheses. While doing so provides a broader evidence base it can introduce confounding cultural factors. For example, New Zealand is lower on key cultural dimensions such as power-distance and individualism than typical countries of comparison (e.g., US, Australia, and the United Kingdom) while it is much lower on power-distance and much higher on individualism than other countries which undertake similar research (e.g., Japan, Indonesia, and China; Hofstede Insights, n.d.). Within the field of disaster research, however, many studies from different countries have consistently identified the same key predictors of preparedness (e.g., Becker et al., 2015) including countries with a history of European colonization (e.g., the United States; Lindell \& Perry, 2000; and Australia; Paton, 2013) as well as countries including Iran (Najafi, Ardalan, Akbarisari, Noorbala, \& Elmi, 2017), Taiwan (Jang, Wang, Paton, \& Ning-Yu, 2016), Japan (Paton, Okada, \& Sagala, 2013), and Indonesia (Sagala, Okada, \& Paton, 2009).

New Zealand's cultural context. New Zealand is a multicultural nation; although most of the population is of European descent, the country has significant populations of indigenous Māori, Pasifika, and Asian ethnic groups. In the Wellington Region, $74.6 \%$ of respondents to the 2018 Census were European/Pākehā, 14.3\% were Māori, 8.4\% were Pasifika, and 12.9\% were from various Asian ethnicities. It is therefore important to consider how members of these various ethnic groups conceptualize hazard risk and preparedness. In particular, te ao Māori (the Māori worldview) does not treat science and culture as separate; for example, stories of tupuna (ancestors) travelling across the Pacific Ocean include aspects of ocean science such as tides and swells (Pihama, Southey, \& Tiakiwai, 2015). Many pūrākau (ancient narratives) include explanations of natural hazard events; storm surges and tsunami are the result of the movement of taniwha, giant, water-dwelling lizards (King, Goff, \& Skipper, 2007), while earthquakes are the movements of Rūaumoko, the son of the sky, Ranginui, and the earth, 
Papatūānuku (McSaveney, 2017). Mātauranga Māori (knowledge and understanding within te ao Māori) allows for the co-existence of traditional, cultural knowledge and colonial science to provide a more holistic view of the relationships between humans and nature, such that Māori knowledge of the environment can contribute important information about hazard risk, response, and recovery (King et al., 2007).

As well as differences in worldview, Māori oral history includes accounts of natural hazard events from centuries before colonization. For example, one tradition tells of an earthquake in approximately $1460 \mathrm{CE}$ which raised the land on which the suburb of Rongotai and Wellington Airport now sit, connecting what was an island, Motukairangi, with the mainland to form Miramar Peninsula. It is possible that this long history of experiencing natural hazard events and dealing with their impacts would also lead to differences in beliefs around risk and preparedness. Unfortunately, the thorough consideration which this possibility warrants is beyond the scope of this thesis. Further, the aim is to identify beliefs shared among the population, across cultures, so that resulting interventions are widely beneficial.

New Zealand natural hazard research-scape. New Zealand has a national research model with an a-typical focus on central government funding. There are three main branches of funding in the area of natural hazard research. First, Crown Research Institutes (CRIs) including GNS Science and the National Institute of Weather and Atmospheric Research (NIWA) are research companies owned by the Crown with the purpose of benefiting New Zealand society. While these CRIs focus predominantly on physical science, they do undertake some work to understand societal impacts of hazards and assess current levels of preparedness. Similarly, the Earthquake Commission (EQC) is a Crown entity which funds research into hazard preparedness and runs public education campaigns.

Second, CRIs and universities collaborate with local Civil Defence Emergency Management (CDEM) groups to establish localized case studies. For example, the AF8 project explores the risk from a Magnitude 8 earthquake on the Alpine Fault which runs for most of the length of the South Island of New Zealand and communicates the potential impacts of such an earthquake to local communities to encourage preparedness. Similar projects include East Coast Life at the Boundary which considers a large earthquake in the offshore Hikurangi subduction zone, Determining Volcanic Risk in Auckland (DEVORA), and It's Our Fault which explored the likelihood of a large earthquake in Wellington and the potential physical and social impacts. The social science aspects of these projects tend to focus on communicating risks and their potential impacts, as well as community-based efforts to increase preparedness, such as facilitating tsunami evacuation walks in coastal communities (Doyle et al., 2020). 
Third, the government funds independent research at academic institutions through a number of platforms, such as the hazards-focused National Science Challenge, Resilience to Nature's Challenges, which has received millions in government funding since 2015, replacing the 10-year Natural Hazards Research Platform. Further, one of New Zealand's Tertiary Education Commission-funded Centres of Research Excellence (CoREs) is dedicated to earthquake research. Many New Zealand universities also partner and collaborate with international research programs, including the Bushfire \& Natural Hazard Cooperative Research Centre in Australia and Washington State Emergency Management in the US.

Social science research in New Zealand published in the last decade focuses predominantly on exploring the impacts of natural hazard events, in particular the 2010-2011 Canterbury earthquake sequence (O’Conner, Johnston, \& Evans, 2011) and the 2016 Kaikoura earthquake (Vinnell, Orchiston, Becker, \& Johnston, 2019). Other research has explored the ways in which New Zealander's perceive risk (e.g., Fraser et al., 2016; McClure, Ferrick, Henrich, \& Johnston, 2019; McClure, Henrich, Johnston, \& Doyle, 2016; McClure, Johnston, Henrich, Milfont, \& Becker, 2015), existing levels of preparedness (e.g., Blake, Tippler, Garden, Johnston, \& Becker, 2018), factors which relate to preparedness and resilience (e.g., Blake, Marlowe, \& Johnston, 2017; Doyle et al., 2018; Gowan, Kirk, \& Sloan, 2014; Kwok, Becker, Paton, Doyle, \& Johnston, 2019; Tuohy \& Stephens, 2016), and the effects of communication decisions such as message framing (e.g., Henrich, McClure, \& Crozier, 2015; McClure, Doyle, \& Velluppillai, 2015; Vinnell et al., 2017, 2018). Many of these studies explore how significant events impact risk perception and preparedness; while this research is vital, it is also important to understand why people choose to prepare without the direct motivation of a recent event. Trends in disaster preparedness in New Zealand suggest that increases in preparedness following large events do not last more than a few months (Colmar Brunton, 2018). Identifying beliefs about the behaviour of preparing which relate to the decision to prepare, regardless of the recency of previous events, can help to improve the effectiveness of public education campaigns in times of quiescence and would therefore represent a meaningful addition to the existing literature.

Further, although some of the existing research explores similar concepts to this thesis, there are few studies which do so with the explicit aim of identifying variables which can be targeted in an intervention or which apply an established behavioural theory. Even fewer examples of published research evaluating interventions exist. As such, the research in this thesis is intended to complement the considerable natural hazard literature produced in New Zealand by using a behavioural theory framework and exploring predictors of behaviour with 
a longitudinal method. Some studies in New Zealand have used behavioural follow-ups (e.g., McClure et al., 2015), pre- and post-test intervention designs (Macdonald, Johnson, Gillies, \& Johnston, (2017), or natural experiments (Vinnell, Milfont, \& McClure, 2019); however, the bulk of the existing literature uses cross-sectional surveys, with some comparisons between geographical locations (e.g., McClure, Henrich, Johnston, \& Doyle, 2016; McClure, Johnston, Henrich, Milfont, \& Becker, 2015) or experimental manipulations (e.g., McClure \& Sibley, 2011; Vinnell et al., 2017, 2018).

\section{Interventions in the Natural Hazard Context}

Public education campaigns in New Zealand. Several organizations have run public education campaigns, primarily aiming to educate New Zealanders about their natural hazard risk and the actions they can take to prepare; such efforts are important but not necessarily sufficient for motivating preparation (Finnis, 2007). Campaigns such as EQC's "Fix, Fasten, Don't Forget" (formerly "Fix, Fasten, Forget") focuses on mitigation actions which can be taken by households such as securing foundations and fastening tall furniture. Recent changes in this campaign, such as the shift from "Forget" to "Don't Forget", suggests an awareness of the reasons why people do not prepare. Similarly, the EQC website now includes separate advice for people in different living situations, including homeowners, tenants, and apartment dwellers, explanations of the benefits of mitigation in terms of limiting damage and injury, and testimonials focused on affective responses to preparing. In late-2016, shortly before the start of this thesis, the National Emergency Management Agency launched a \$2.5 million, threeyear public education campaign: "Never Happens? Happens" (Kaye, 2016). This campaign used a website, online videos, social media, and school programmes to increase preparedness for emergencies with the key tool of highlighting past natural hazard events and emergencies to counter the New Zealand "she'll be right" attitude: the belief that devastating events have not often occurred in the past and therefore will not occur in the future. A slight increase in national levels of household disaster preparedness between 2016 and 2017 is somewhat confounded by the 2016 Kaiōura earthquake, but levels returned to pre-2016 levels by 2018 (Colmar-Brunton, 2018).

Another avenue used for public education is museums. Since the national museum, Te Papa Tongarewa, opened in 1998, it has featured an exhibit called "Awesome Forces" which explains New Zealand's seismic and volcanic activity and included the "Edgecumbe earthquake house". This was a small structure built on a movable foundation so that museum visitors could experience a simulation of earthquake shaking. This exhibit was altered and reopened in 2019; the updated earthquake house now includes a video which has a more explicit 
focus on preparation rather than simply risk, such as actors demonstrating protective actions to take during earthquake shaking.

Interventions to increase natural hazard preparedness in New Zealand often use community-based approaches, rather than leveraging the broad scale of most public education campaigns (Finnis, 2007). For example, the 2001 Shake Safe Initiative installed hot-water cylinder restraints in 350 homes in the Wellington area and provided some educational material. This programme was deemed a success, despite there being no formal evaluation, and intentions to extend it nationally were not fulfilled (Finnis, 2007). Historically, there has been a tendency to declare interventions a success either if their implementation is completed or if the participants are satisfied (Finnis, 2007). While those 350 homes were safer to a small extent, it is unclear whether receiving external support for one mitigation action motivated the household to undertake further preparation. The one type of intervention in New Zealand which has received some formal evaluation is drills, mainly for earthquakes and tsunami (e.g., Coomer, Tarrant, Hughes, \& Johnston, 2012; Johnston et al., 2011; Johnston et al., 2016), though these evaluations focus on schools and more on knowledge and logistics than cognitive or behavioural outcomes. As a result of the lack of intervention studies in New Zealand, the following section will also draw on international literature.

Natural hazard preparation interventions. Research into natural hazard preparation has identified demographic or individual difference factors which are difficult, if not impossible, to manipulate in an intervention (Crawford et al., 2019; Lindell \& Whitney, 2000). For example, age and gender are inconsistently found to relate to preparedness, both in New Zealand (Becker et al., 2015) and globally (Lindell \& Perry, 2000; Solberg et al., 2010). Solberg et al. (2010) in their review of the literature conclude that demographic variables likely relate to risk perception rather than preparation; while perception of a risk is necessary for someone to prepare for that risk it is not a predictive factor (Bourque, 2013; Bourque et al., 2012). Further, while an intervention could be tailored for a specific gender or age group, doing so reduces the efficiency of such an effort.

Similarly, previous research consistently demonstrates that experience of past events relates to earthquake preparation (e.g., Becker et al., 2015; Solberg et al., 2010; Vinnell, McClure, \& Milfont, 2017). However, this association is not found consistently (Bourque et al., 2012; Lindell, 2013) and likely acts via risk perception (Lindell \& Hwang, 2008). Impacts of experience on preparation therefore depends on the type of experience and tends to be parabolic, where moderate levels of experience tend to positively relate to preparation, but low and high levels are either negatively related or unrelated to preparation (Solberg et al., 2010). 
While hazard experience can be effectively manipulated using tools such as survivor testimonials in smaller-scale, more intensive interventions (e.g., Parent \& Fortin, 2000), this research aims to identify strategies which can be used efficiently (i.e., broadly and nonintensively). The optimum level of experience to drive preparation is likely to differ between individuals and is therefore difficult to attain using a broad public education campaign.

Risk perception, another commonly cited prerequisite for preparation (Paton \& Johnston, 2001), poses similar challenges. If an individual does not perceive enough risk from a natural hazard, then they are unlikely to act to mitigate that risk. Similarly, if an individual perceives the risk as too high, they can become fatalistic (i.e., believe that preparing will not help) and again not act; such barriers are resistant to traditional methods of encouraging preparation (Paton \& Buergelt, 2019). Research suggests that most residents in Wellington know about the risks they face (in particular from earthquakes; Johnston et al., 2013; Khan et al., 2012), that risk perception is not typically directly linked with preparedness (Bourque, 2013), and that residents know at least some actions they could take to be better prepared (McClure et al., 2015). Previous public education campaigns in Wellington have increased awareness of the risk that natural hazards pose to the city but have had little impact on overall preparedness (Johnston et al., 2013), reflecting the low impact of such campaigns in other global contexts (Lindell \& Whitney, 2000; Paton et al., 2005).

One possibility for the lack of direct causal effects of risk perception on preparation is that previous research is not examining the concept with the correct method to identify such a direct effect. For example, the relationship between risk perception and preparation is likely not linear, where a moderate level of perceived risk can be motivating but both too low and too high a level can be inhibiting (e.g., Becker et al., 2017; Solberg et al., 2010). It is possible that analyses assuming a linear relationship inaccurately indicate that there is no direct causal influence.

There are many other factors which can either encourage or discourage individuals from preparing for events such as earthquakes. Research suggests that perception of risk influences survival actions while the belief that one can reduce earthquake damage influences mitigation actions (McClure et al., 2015; Spittal et al., 2008). Similarly, neuroticism correlates with preparation actions to help survive but not to mitigate disaster impacts (Heller, Alexander, Gatz, Knight, \& Rose, 2006). McClure et al. (2015) found that people judged cost as important for only the four most expensive out of 12 actions to prepare for earthquakes; cost was typically less important than thinking about the risk and preparing, prioritizing preparing, and thinking preparation is necessary. The authors commented on the need to reinforce what actions can be 
taken as well as emphasizing that those actions are effective at reducing damage. For example, presenting images of distinctive damage leads to attributions of the cause of that damage to poor engineering which is seen as more controllable (McClure, Sutton, \& Sibley, 2007).

The primary aim of this thesis is to identify beliefs and cognitions related to household preparation for natural hazards which are widely held and able to be modified with relatively simple strategies. These strategies can then be applied efficiently to existing public education campaigns in the region, nationally, and internationally. Often campaigns focus on information around risks and actions, with some small tasks such as checklists to create engagement. Authors in the field of behaviour change argue for the importance of using a research-based model of behaviour change to inform intervention strategies (e.g., Ajzen, 2011a; Joffe, PerezFuentes, Potts, \& Rossetto, 2016). Below is a brief review of a selection of these interventions to highlight the gap, both in research and in practice, which this thesis intends to address. The theoretical framework used in this thesis to extend beyond previous interventions is discussed in the next chapter.

Interventions to increase natural hazard preparation seldom include reported evaluations of the effectiveness of the intervention, with few examples of published research even providing enough information around the intervention method for replication (Joffe et al., 2016). To address this issue, Joffe et al. (2016) evaluated the fix-it intervention, which involves a six-hour workshop focused on securing items around the house to reduce damage and injury in an earthquake. The authors provide sufficient detail for the intervention to be re-created, so that a consistent, widely used intervention could be established. Although they did observe an increase in household preparedness following the workshop, the control group also showed increased preparedness. The authors attribute this increase to the impact of researchers visiting the homes of participants to check behaviour. At a 3-month follow-up, both groups had increased their preparedness significantly from baseline and did not differ from each other.

Despite the value of this research, this intervention may have limited real-world applicability. While conducted in the field, the incentives for participation are unrealistic for a broader implementation of the intervention; for example, participants received between US\$180 and US\$330 for taking part in the study. Such large financial incentives can introduce sampling biases (Sharp, Pelletier, \& Lévesque, 2006). The disproportionate number of unemployed participants (48\%) in the study suggests that the large incentive might have created a self-selected sample of people motivated by financial gain (Singer \& Couper, 2008). Removing or reducing the incentive necessary for a wide-spread implementation of this intervention would likely dramatically reduce participation (Sharp et al., 2006). While the study 
by Joffe et al. (2016) shows some evidence that the intervention strategies employed could be effective, it may not demonstrate an intervention which could be both effectively and efficiently run with larger portions of the population.

Orchiston, Manuel, Coomer, Becker, and Johnston (2013) tested the ShakeOut earthquake drill, developed in California, with residents of New Zealand's West Coast, which is at significant risk of an event on the large Alpine Fault. The purpose of the ShakeOut drill is to educate people on the protective actions they should take during earthquake shaking. ShakeOut has since been run at a national level in New Zealand with hundreds of thousands of people participating each time (Tipler, Tarrant, Johnston, \& Tuffin, 2016). Among the original West Coast participants, approximately two-thirds of families of school children did not have an emergency kit. These school children typically knew that an earthquake along the Alpine Fault was likely, but they did not think it would happen during their lives (Orchiston et al., 2013). Tipler et al. (2016) suggested that it is important to use drills as an opportunity to also link risk knowledge to preparation behaviour. They found that the ShakeOut drill did increase preparation in the homes of the school children, as well as increasing knowledge and awareness.

Although ShakeOut is a widely implemented intervention in New Zealand, it has not been thoroughly evaluated in terms of cognitive and behavioural impacts. Such an evaluation study is included in this thesis, introduced briefly at the end of this chapter and presented as Study 4 (Chapter 6). Before proposing a new intervention, it is logical to test the effectiveness of existing ones which can then be repeated and improved, as opposed to the continual development of new interventions (Joffe et al., 2016).

Two studies evaluating education interventions have been conducted in conjunction with the "Awesome Forces" exhibit at Te Papa Museum mentioned earlier. MacDonald, Johnson, Gillies, and Johnston (2017) provided information to visiting school children on how to prepare their homes for an earthquake and gave some of them a fill-in plan, plastic putty, or steel brackets to take home and use. Those who received the plan, putty, or brackets showed better knowledge of how to prepare, as did their parents, than those in the control group who did not receive a take-home preparation tool. However, evidence for increases in behaviour was limited.

Similarly, Gampell, Gaillard, Parsons, and Le Dé (2020) ran EQC's “Quake Safe House" game inside the exhibit. This game asks players to prepare a home in Wellington for an earthquake by applying preventative measures. No information about the purpose of the measures is provided, though players receive feedback after a simulated earthquake in the form 
of a tick for a measure used in the right place and a cross for a measure used in the wrong place. While most participants felt like they gained knowledge, only 9 out of 22 recalled new mitigation strategies they learned from the game. Feedback indicated a need for more information about why a measure was appropriate and how it functioned to reduce damage.

\section{Domain-Specific Definitions of Key Terms}

This thesis uses social psychological theory to understand the motivators and inhibitors of individual preparation for natural hazards. This aim is important at a local, national, and global level, with meaningful applications for practice as well as implications for the implementation and measurement of theory. To explain this importance, the current chapter has introduced the context for this research, narrowing down from the broadest level (global) to the narrowest (local: the specific research context). Given that this thesis aims to contribute to a global conversation, I will briefly define the main non-psychological terms which will be used throughout this thesis.

The following definitions are based on those decided in a working group report organized by the United Nations General Assembly. The UN's disaster risk reduction branch, the United Nations Office of Disaster Risk Reduction (UNDRR), is a global organization examining disaster risk and therefore offers an authoritative source to provide consistent and accepted definitions. However, several versions of the glossary provided by the UNDRR since its initial document in 2004 offer meaningfully different definitions. The following are from the latest version (United Nations General Assembly, 2016) which retained some weaknesses from previous versions by including technical jargon (Kelman, 2018). However, the definitions presented below should be sufficient for understanding these concepts in the context of this thesis.

Disaster. A disaster is defined as "A serious disruption of the functioning of a community or a society at any scale due to hazardous events interacting with conditions of exposure, vulnerability and capacity, leading to one or more of the following: human, material, economic and environmental losses and impacts." (United Nations General Assembly, 2016, p. 13). Put simply, a disaster happens when a natural event, such as an earthquake, occurs near an inhabited area and the impacts exceed the ability of humans, structures, and/or infrastructures to cope, leading to serious negative outcomes. Disasters can be small- or largescale, frequent or infrequent, and slow- or sudden-onset. Of primary concern in this thesis are disasters of medium scale (i.e., with impacts that exceed the response capabilities of local communities), infrequent (i.e., disasters which do not occur regularly, such as devastating earthquakes, rather than disasters which occur more often and more predictably, such as severe 
flooding occurring most years during wet seasons), and sudden-onset (i.e., disasters which occur suddenly, with little warning, such as tsunami, rather than disasters which are the result of gradual processes such as sea-level rise).

Hazard. A hazard is defined as "A process, phenomenon or human activity that may cause loss of life, injury or other health impacts, property damage, social and economic disruption or environmental degradation." (United Nations General Assembly, 2016, p. 18). A hazard, such as a natural event like an earthquake, is necessary but not sufficient for a disaster to occur. If communities are sufficiently equipped to deal with hazard impacts, then disasters are not likely. As with disasters, the types of hazards faced are diverse. They can be natural (i.e., a natural process or phenomenon, such as a tsunami), anthropogenic (i.e., caused by humans, such as a terrorist attack), or socio-natural (i.e., an interaction of both natural and human causes, such as the Fukushima Daichii nuclear incident). This thesis focuses on natural hazards, primarily those which are geophysical (e.g., earthquakes). These types of hazards are perceived differently to other natural hazards, such as those which are biological in nature (e.g., pandemics), and different from anthropogenic hazards (Henrich, McClure, \& Doyle, 2018; Kahlor, Olson, Markman, \& Wang, 2018).

Disaster risk. To prepare for a disaster, the risk of such an event must be perceived (e.g., Solberg et al., 2010). Disaster risk is a function of the likelihood of an event occurring and the severity of the negative outcomes should one occur (Brundl, Romang, Bischof, \& Rheinburger, 2009). For example, the risk of seasonal flooding is different to that of the risk of damaging earthquakes. The former has a higher probability of occurring, but it also tends to produce less severe outcomes than the latter.

Disaster risk reduction. The reduction of disaster risk is typically seen as the goal for disaster-related research and practices. The aim is to avoid creating new risks, such as better identifying hazard-prone areas or improving the standards of new buildings, and reducing extant risks, such as retro-fitting vulnerable buildings and increasing the preparedness of individuals. As this goal is complex and expansive, the current thesis narrows the focus on reducing disaster risk to increasing household preparedness, so that individuals are better equipped to survive during, and recover after, a natural hazard event.

\section{Disaster Terminology and Framing Effects}

Some researchers in the field of disaster risk reduction make the argument that the term "natural disasters" is inappropriate, as disasters only occur due to decisions made by humans, such as to live in an area prone to natural hazards or to occupy poorly-constructed buildings (e.g., Blanchard, 2018; Kelman, 2018; Ras, 2017). A similar argument is taking place within 
the area of climate change; messages referring to climate change, rather than global warming, lead to stronger beliefs and intentions to act (although only for political independents; Benjamin, Por, \& Budescu, 2017). Similarly, perceptions of the urgency and risk around climate change, as well as willingness to act, can be increased by referring to the war, rather than the race, against climate change (Flusberg, Matlock, \& Thibodeau, 2017).

Although academic debate about the term "natural" disaster started many decades ago, with the argument itself extant for hundreds of years (Chmutina \& Meding, 2019), limited empirical research has approached the topic. Messages attributing earthquake damage to human causes such as building design and emphasizing distinctive damage lifts perceptions that this damage can be prevented (McClure et al., 2007; McClure, Allen, \& Walkey, 2001). However, there is no direct evidence that referring to natural disasters is detrimental to perceptions, attitudes, and behavioural intentions.

One argument against the term natural disasters proposes simply dropping the "natural" classifier and referring solely to disasters (e.g., Raz, 2017). According to this argument, this would have the desired effect of leading people to attribute outcomes to human causes, and therefore perceive this damage as preventable (e.g., Blanchard, 2018), rather than putting the focus on nature as is prominent in public, media, and political discourse (Solberg et al., 2010). This phrasing is also common within research; Chmutina and von Meding (2019) analysed 589 research papers using the term "natural disaster". The clear majority of papers used the term simply because it is prominent in the field. Only a minority of papers (13) argued for the inappropriateness of the term. Many researchers within the field of disaster risk reduction seem to only debate the ramifications of term within the academic discourse, assuming what other researchers intended by their use of the term and not considering the impact of terminology used in communication with the general public. Although it may be logical to assume that including "natural" leads to assignment of blame to nature, and that this therefore impacts behaviour, such assumptions should be supported empirically.

An argument against dropping the "natural" classifier emphasizes the changing meaning of disaster. Over recent decades, the threshold for an event being a "disaster" has shifted, so that disasters can include more common events such as minor misfortunes and accidents (Furedi, 2007). The term can therefore lead to confusion particularly among nonresearchers (Kelman, 2018). Furedi argues that "As a growing range of human experiences are associated with disasters, the distinction between normal daily life and a disaster becomes ill defined...disaster ceases to possess any distinct features" (Furedi, 2007, p. 487). As well as the increasing number of definitions of the term “disaster" (Maynor \& Arbon, 2015), a range of 
synonyms for disaster have emerged, such as "emergency", with no consensus about differences in meaning (Kelman, 2018). The "natural" classifier, therefore, serves an important purpose of distinguishing disasters due to natural causes from events which would not historically have been labelled disasters as well as catastrophic events due to other causes. Risks solely caused by humans such as nuclear plant meltdowns (Rochford \& Blocker, 1991) lead to more negative affect (Kahlor et al., 2015) and are seen as more voluntary, more in need of management, and more of a public responsibility (as opposed to a private responsibility) than those disasters associated with natural hazards (Brun, 1992).

Natural disasters, therefore, appear to represent a distinct set of risks and outcomes. Removing the "natural" part would lose the distinction from other types of disasters and could lessen the impact of the term due to its broader scope. An alternative to removing the natural classifier is to change the "disaster" part. After "natural disaster", the most common term used by agencies and media to refer to a range of risks and events involving natural processes is "natural hazards". This retains the reference to nature, to ensure people differentiate these risks from technological or biological ones, while addressing the issues with the term "disaster" due to its shifting meaning. Theoretical reasons for differences in how these terms are perceived are presented later, in the context of the findings of Study 2 which compared responses to preparation-related judgments between the two phrases.

\section{Aims of the Thesis}

This thesis has two main aims. The first is to refine and improve the conceptualization and methodology of the key theory used: the Theory of Planned Behaviour. Details of this aim are provided in the following chapter after the theory is reviewed. The second and primary aim is to identify cognitions and their theorized antecedent beliefs that lead individuals to prepare their homes for a disruptive natural hazard event. The population under study is the urban Wellington region of New Zealand, consisting of Wellington City, the Hutt Valley, Johnsonville, Tawa, and Porirua (see Figure 1.1). As described above, Wellington is prone to natural hazard events and vulnerable to their impacts. Many residents are aware of these risks, but the majority are not well prepared (Khan et al., 2012; Johnston et al., 2013; ColmarBrunton, 2018). 


\section{Urban/Rural Profile Categories: Wellington Region}

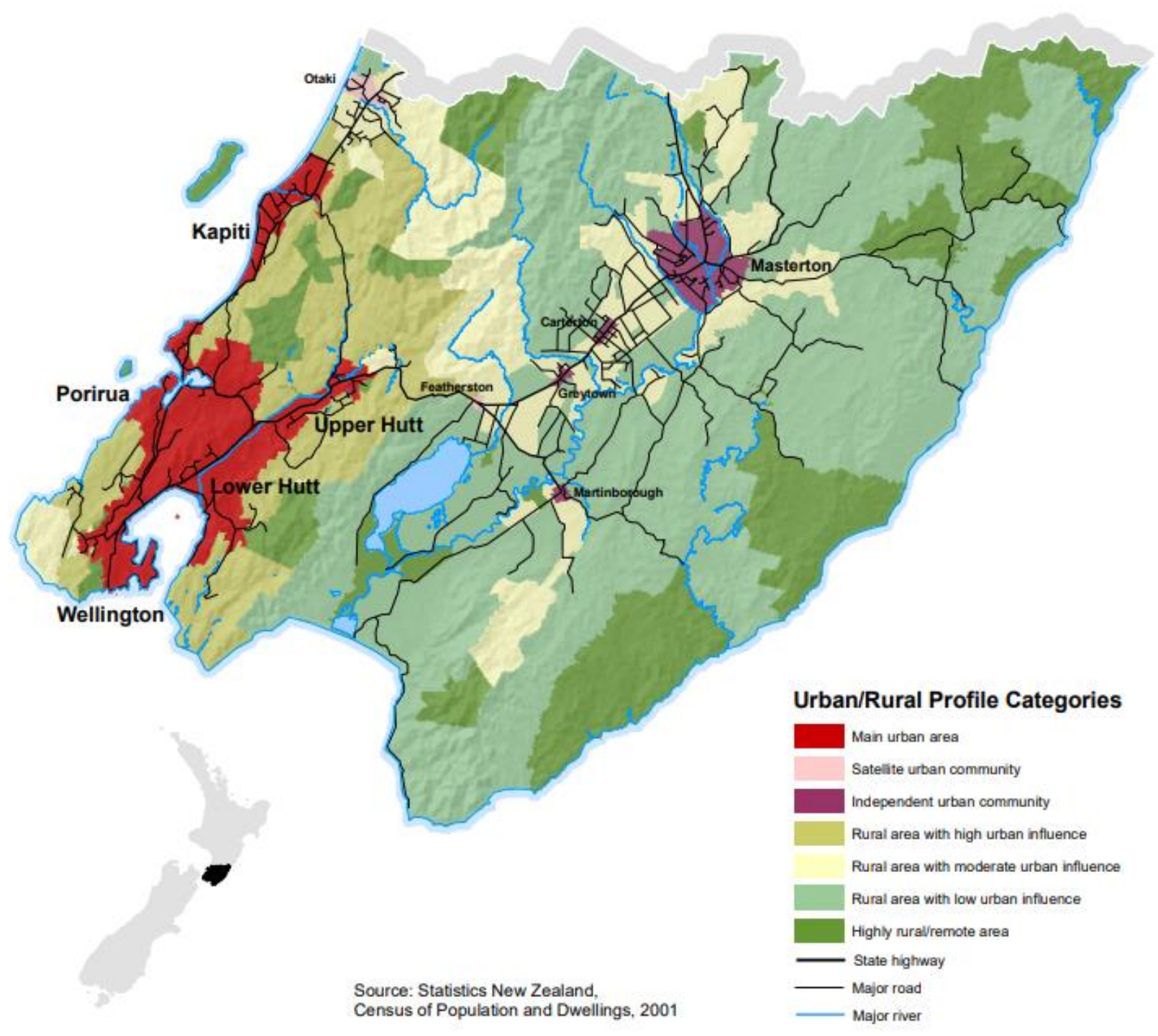

Figure 1.1. Map of the greater Wellington region of New Zealand's North Island. The study area for this thesis includes the main urban areas in red, excluding Kāpiti (Stats NZ, 2002). Source: $\underline{\text { Stats NZ and licensed by Stats NZ for re-use under the Creative Commons Attribution }}$ 4.0 International licence. 


\section{Overview of the Research}

The first study of this thesis confirms previous findings of both low levels of preparation in Wellington as well as the prevalence of internal rather than external barriers; that is, people report not preparing because of factors relating to their behaviour (e.g., simply not getting around to it) rather than factors relating to their situation (e.g., cost). Primarily, this research will test The Theory of Planned Behaviour, including six different concepts which are potentially related to intentions to prepare, and therefore behaviour. The most relevant and influential factors from this theory will then be proposed as targets for public education campaigns run throughout the region, with the goal of suggesting improvements to such campaigns or inclusions for new interventions throughout New Zealand and overseas. Studies 1,2 , and 3 build from a preliminary test of the applicability of the theory to the concept to a full, longitudinal application of the theory to predicting natural hazard preparation. Finally, Study 4 used the same theoretical framework to retrospectively evaluate a campaign run previously by New Zealand's National Emergency Management Agency, the 2018 New Zealand ShakeOut drill. A more detailed overview of the studies is presented at the end of the next chapter, following the introduction and discussion of the central theory used in this thesis.

\section{Summary}

Natural hazards are increasingly impacting people both nationally and globally. A vast amount of research has contributed to an understanding of what factors lead to people preparing for such events, however there is a clear gap for research which identifies specific and changeable beliefs. The following chapter introduces the theoretical framework used in this research to achieve this aim, as well as the reasons for, and intended contributions of, developments to that theory. 


\section{Chapter 2: The Theory of Planned Behaviour}

\section{Chapter Overview}

Given the complexity of the factors which contribute to preparation for natural hazards, it is important for research and interventions to be informed by theory, founded on empirically established associations, and properly evaluated (Joffe, Perez-Fuentes, Potts, \& Rossetto, 2016). This chapter outlines several relevant theoretical models and then reviews the theory applied in this research: the Theory of Planned Behaviour (TPB; Ajzen, 1991). The TPB has been used extensively in other research domains such as health psychology with meta-analyses of studies examining behaviours such as eating (McDermott et al., 2015; Riebl et al., 2015) and smoking (Topa \& Moriano, 2010) but has rarely been applied to the area of hazard-related behaviour (Ejeta, Ardalan, \& Paton, 2015). The TPB is used in this research to test factors which explain intentions to prepare and could be targeted in future behaviour change campaigns. A detailed explanation and review of this theory is given later, followed by a discussion of weaknesses in the theory and issues with previous methodology which this thesis aims to address. First, other behavioural theories which have been used in natural hazards research are described to offer an explanation as to why they were not applied here.

\section{Theories in Natural Hazard Research}

This section briefly introduces some of the more prominent theories used within the natural hazard literature (for other overviews of theories relevant to natural hazard preparation, see Beatson \& McLennan, 2011; Lindell \& Perry, 2000; Lindell \& Whitney, 2000; Paton, 2019). Brief comparisons between these theories and the one used in this thesis are drawn at the end of the section. The theories introduced here are: Social Cognitive Theory, the Personrelative-to-Event model, Protection Motivation Theory, the Protective Action Decision Model, and Community Engagement Theory. These theories are presented in order of relevance from lowest to highest, which roughly approximates chronological order. The review of these theories is intended to be critical in the sense of highlighting the specific contexts in which the theories are most useful, so as to explain why they were not the most appropriate theories to use for the research aims and context of this thesis. None of the theories were disregarded due to general methodological or conceptual flaws, therefore an in-depth review of limitations along those lines is not presented. Other theories less often applied and less specific to the field are not reviewed here (see Ejeta et al., 2015). 
Social Cognitive Theory. Social Cognitive Theory (Bandura, 1986) proposes that an individual's behaviour is determined by personal factors, such as cognitions, and factors within their environment, such as social influence. However, this theory also proposes that the relationships between these three factors (the individual, their environment, and their behaviour) are reciprocal; for example, an individual's thoughts influence behaviour and behaviour influences thoughts. This triadic reciprocal determinism (Bandura, 1989) leads to a complex model in which cause and effect is difficult to determine. As a result, identifying simple ways in which to influence behaviour is not a straightforward exercise. While a key theory within social psychology, and with some application to natural hazard preparedness (e.g., Beatson \& McLennan, 2011; Najafi, Ardalan, Akbarisari, Noorbala, \& Elmi, 2017), the theory covers a broad range of factors involving the concepts of cognition and environmental influence. This means that additional research is required to determine which of these factors should be tested for relevance, as it is difficult to test all proposed factors within a single model.

Person-relative-to-Event Model. The Person-relative-to-Event model (Duval \& Mulilis, 1999) emphasizes the outcome of problem-focused coping, which relates to intentions to prepare (e.g., McIvor \& Paton, 2007), compared to emotion-focused coping. According to this approach, the extent to which people engage in problem-focused coping depends on their perception of whether the resources available to deal with a threat are sufficient or insufficient. According to the model, if people perceive the resources available to deal with a threat are sufficient considering the magnitude of that threat, they are more likely to engage in problemfocused coping, and less likely to engage in problem-focused coping if the resources are perceived as insufficient. The PrE model has been used in a limited range of hazard and cultural contexts, with partial success to explain preparedness for tornadoes (Mulilis \& Duval, 1997) and earthquakes in the US (Mulilis \& Duval, 1995; Duval \& Mulilis, 1999). While the similarities in findings around disaster preparedness in New Zealand and the US suggests that a model which functions in the US would be applicable in New Zealand, other theories have demonstrated validity across a wider range of international contexts and therefore would likely produce more generalizable findings.

Further, the model implies, but does not make explicit, a positive association between problem-focused coping and preparation behaviour (Mulilis \& Duval, 1995). This lack of behaviour as the key outcome in the model means that studies using the PrE are limited in their ability to test whether its factors predict behaviour, and therefore whether an intervention based on targeting those factors would likely be effective. Other theories exist which have been used across a larger number of studies and contexts, consider behaviour explicitly, and include 
factors which are more likely to be successfully and effectively targeted in an information campaign.

\section{Protection Motivation Theory.}

SOURCES OF
INFORMATION
COGNITIVE MEDIATING

PROCESSES
INTERMEDIATE

STATE

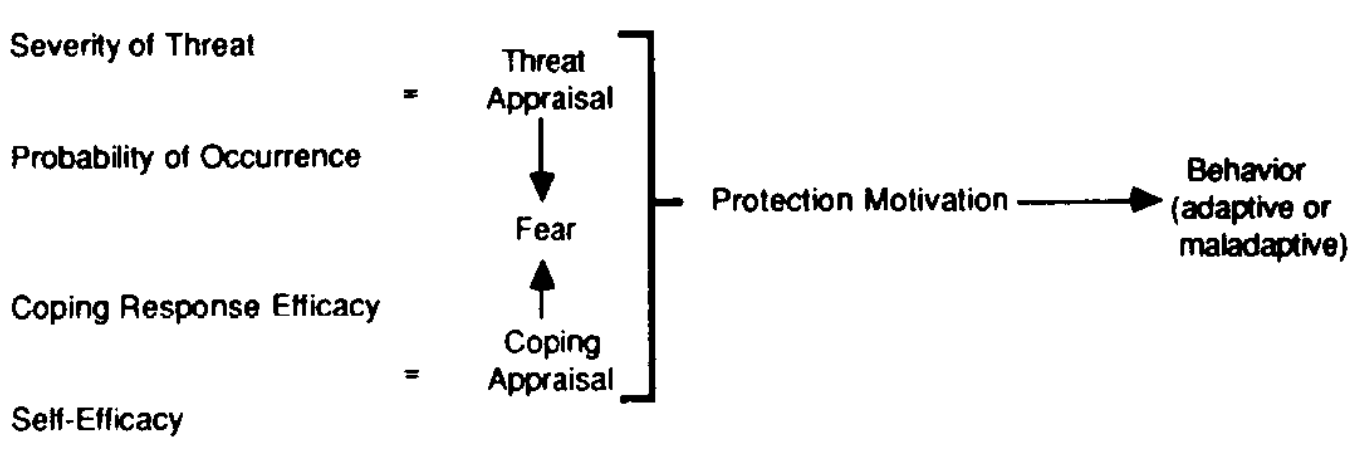

Figure 2.1. Protection Motivation Theory (PMT; Tanner, Day, \& Crask, 1989)

Protection Motivation Theory (PMT; Figure 2.1; Tanner, Day, \& Crask, 1989) proposes mechanisms by which individuals deal with threats: namely, how they appraise threats in terms of the danger they pose and how they appraise their ability to cope with that threat. As with PrE, if people perceive a threat and perceive that they have the capability to deal with that threat, then they are more likely to engage in protective actions. However, this model explicitly includes the key concept of an individual's behavioural capability as well as the importance of resources, presenting a key strength over the PrE in that the theorized predictors of behaviour are more specific and therefore applications of the model would likely be more informative. Unlike PrE, PMT has been used to explain behaviour in a number of hazard and cultural contexts, such as earthquake preparedness in Taiwan (Tang \& Feng, 2018), bushfire preparedness in Australia (Westcott, Botzen, Bambrick, \& Taylor, 2017), and flooding in France and Germany (Bubeck, Botzen, Laudan, Aerts, \& Thieken, 2017), as well as many uses in the area of pro-environmental behaviour (Kothe et al., 2019). The explicit inclusion of 
behaviour as an outcome is a further strength of this model, though studies using PMT often do not consider the intention-behaviour relationship (Kothe et al., 2019).

As with PrE, this model is founded on a history of using fear-arousing and negative threat appeals to increase behaviour, dating from the 1950s. In Wellington, the current research context, there is high awareness of the risk posed by natural hazards (Johnston et al., 2013); this awareness of the existence of natural hazard risk, as well as understanding of potential impacts, is generally high across New Zealand (93\% and 86\%, respectively; Colmar-Brunton, 2018). Attempts to manipulate this variable among residents of Wellington and New Zealand broadly would therefore likely encounter ceiling effects which would limit the usefulness of the construct both as a predictor and as an intervention target. Further, risk perception is difficult to manipulate successfully as the optimum level, where risk is perceived as neither too low nor too high, is highly individual (Solberg, Rossetto, \& Joffe, 2010) and efforts to shift perceptions can backfire. For example, an information campaign educating about volcano risk in New Zealand decreased concern about that risk among a considerable portion of the study sample (Ballantyne, Paton, Johnston, Kozuch, \& Daly, 2000). It is possible that in more targeted interventions, where existing risk perceptions are consistently lower among the targeted audience, fear appeals as proposed by the PMT might be effective; however, the aim of this research is to inform broad interventions which cannot be targeted in the same way. Further, meta-analyses of studies reporting interventions to increase appraisals of risk and threat have been shown to have small or non-significant effects on behaviour when responseefficacy and self-efficacy are not high (Peters, Ruiter, \& Kok, 2012; Sheeran, Harris, \& Epton, 2013). Peters et al. (2012) suggest using threat communication after high levels of efficacy have been established, while the findings of Sheeran et al. (2013) suggests communicating threat and increasing efficacy concurrently. However, the existing high levels of risk perception in Wellington and New Zealand suggests that it is likely more efficient to focus on factors which translate that perception into behaviour, such as efficacy.

The second component of the model, coping appraisal, concerns whether individuals are aware of a way to cope with the threat. Unlike PrE, PMT explicitly models the mechanisms of resource appraisal: response-efficacy and self-efficacy. These two factors have been repeatedly associated with preparation in New Zealand and internationally (Becker et al., 2015; Johnston et al., 2013; Lindell \& Whitney, 2000; Paton, 2013; Paton et al., 2005) and therefore present potential targets for intervention. However, other models also include behaviour, response-efficacy, and self-efficacy along with several other factors more relevant to this particular research context where risk perception is known to be high on average. Further, 
studies using PMT do not consistently include all constructs of the theory and use different operationalizations and there is a lack of empirically successful intervention techniques to target the factors included in the model (Kothe et al., 2019). Therefore, a more novel and distinctive approach would offer additional important factors which have not been considered to the same extent as those included in Protection Motivation Theory but which have been tested in evaluated interventions.

\section{Protective Action Decision Model.}

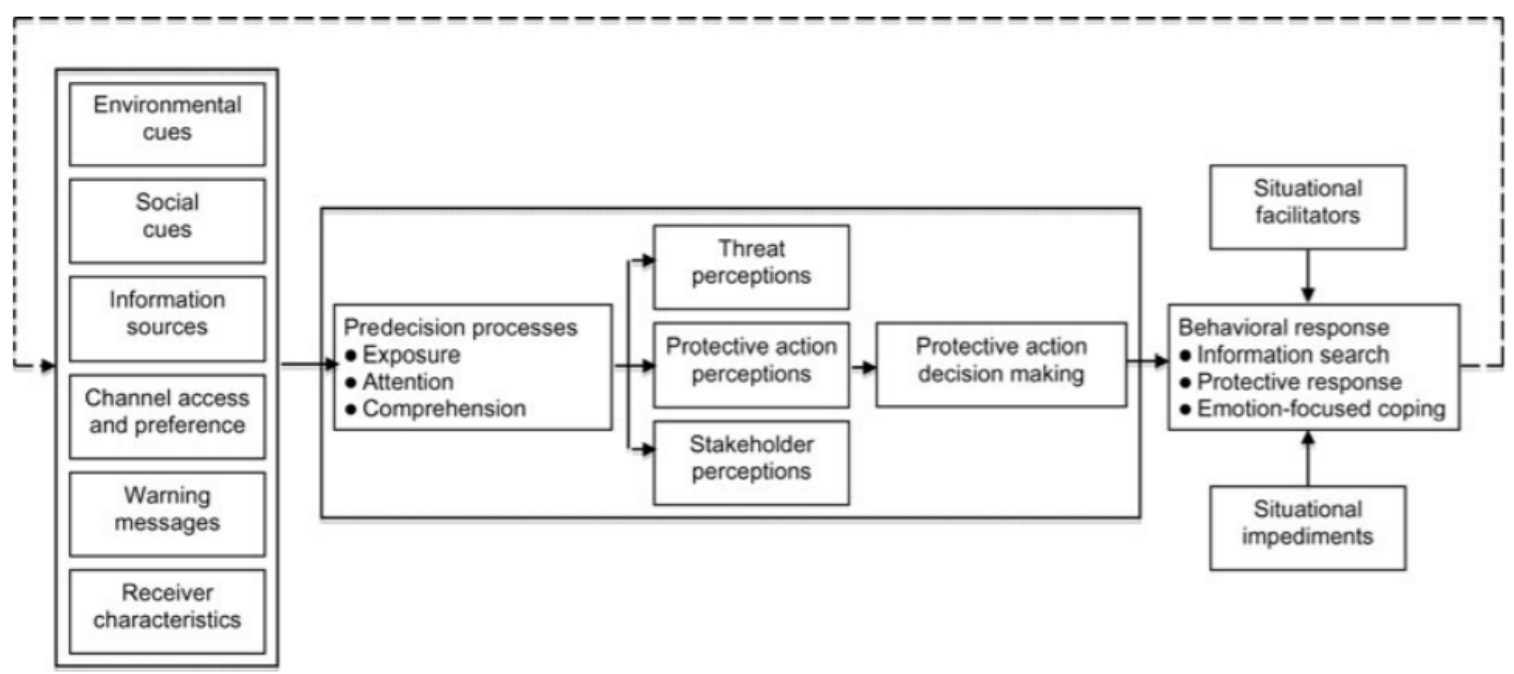

Figure 2.2. The Protective Action Decision Model (PADM; Lindell \& Perry, 2012)

The Protective Action Decision Model (PADM; Figure 2.2.; Lindell \& Perry, 2012) includes social and cognitive components in line with Social Cognitive Theory but was developed specifically in the field of natural and environmental hazards. This model has been used to explain preparedness for a number of hazards across cultural contexts, including US studies exploring intentions to evacuate following an accidental chemical release (Heath, Lee, Palenchar, \& Lemon, 2018) and actual evacuation behaviour in a historical nuclear power plant crisis (Houts et al, 1984), flood preparedness in the Netherlands (Terpstra \& Lindell, 2012), and use of electric vehicles during a smog crisis in Beijing (Liu, Ouyang \& Cheng, 2019).

This model has a strong focus on the use of information from a variety of sources (e.g., environment, social groups, and warning messages). Exposure and attention to, and comprehension of, this information influences key perceptions (namely, of the threat and of protective actions), which then influences behaviour. This model also shares similarities with 
Protection Motivation Theory in that it incorporates appraisals of the threat and of ways to deal with that threat. The key differences are that the PADM proposes distal causes of these appraisals (information sources) and includes situational barriers and facilitators. However, the model is intended to focus on behaviour in terms of decisions to evacuate or shelter in place once a hazard event has commenced; these actions are fundamentally different to behaviour which require maintenance, differ in how they are completed, and require behavioural decisions which often need to occur in a matter of minutes (Lindell \& Perry, 2012). Further, applications of the PADM typically centre on a "focusing event" (Lindell \& Perry, 2012). While the contexts in which the PADM has been successfully applied suggests that the model can be useful for survival and mitigation actions, which are the focus of this research, these studies often selectively include the factors which are relevant for the behaviour under study. This means that significant factors are likely to either be determined in a post-hoc fashion based on exploratory analyses where the likelihood of finding significant relationships is inflated by virtue of conducting a large number of comparisons, or in an a priori fashion which means that factors are likely picked based on existing evidence, which limits the potential for novel contributions. That different studies choose to include different factors from the theory in statistical models also means that comparing findings between studies is more difficult and less informative than when the same set of factors are used in the same way.

While this model offers a useful structure for understanding the flow-on effects of information, there is little scope for a simple, broadly-applicable intervention to address the most distal behavioural causes proposed by the model. Further, the situational factors proposed in this model, while they can contribute to predicting behaviour, typically produce mixed findings (e.g., Bourque et al., 2012; Lindell \& Perry, 2000; Solberg et al., 2010) and are difficult, if not impossible, to manipulate. Finally, this model encounters the same problems as Protection Motivation Theory regarding the actual predictive and change capability of threat perceptions. Therefore, a theory with less focus on risk and threat appraisals and on evacuation behaviour, as well as one which includes targetable cognitive factors, was preferred. 


\section{Community Engagement Theory.}

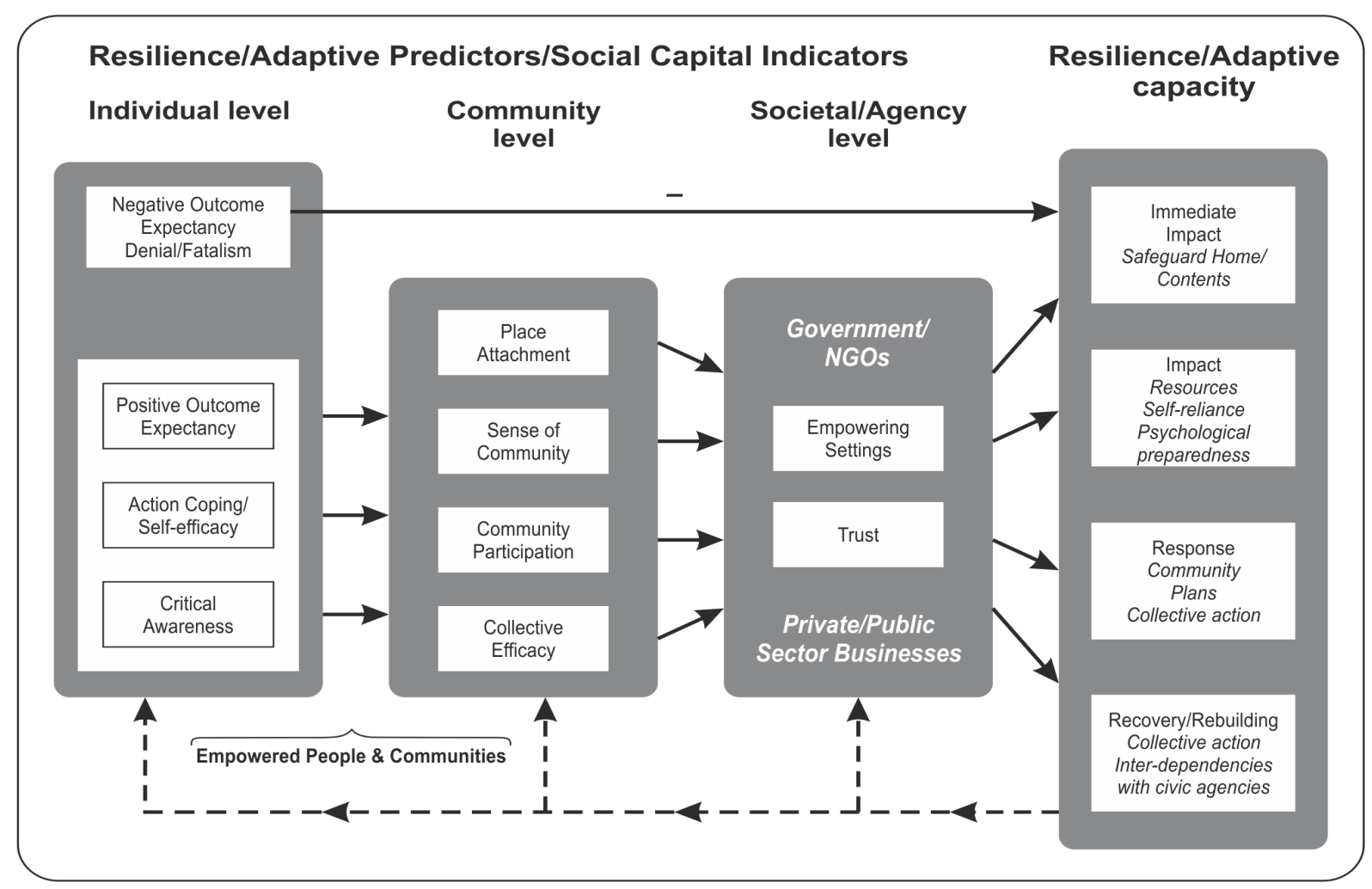

Figure 2.3. Community Engagement Theory (CET; Becker, Paton, \& Johnston, 2015)

Finally, Community Engagement Theory (CET; Figure 2.3; Paton, 2013) comprehensively describes many factors which have been shown to relate to natural hazard preparation. This model has been validated in a number of hazard contexts including tsunami, earthquakes, wildfires, and pandemics (Paton, 2013) as well as in diverse countries including Iran (Najafi, Ardalan, Akbarisari, Noorbala, \& Elmi, 2017), Taiwan (Jang, Wang, Paton, \& Ning-Yu, 2016), Japan (Paton, Okada, \& Sagala, 2013), and Indonesia (Sagala, Okada, \& Paton, 2009). This theory proposes that the main antecedent of preparation is an individual's beliefs about the outcome of preparing, termed outcome expectancy, which can be positive (i.e., preparing will reduce damage from a natural hazard event) or negative (i.e., impacts will be too severe to mitigate). While positive outcome expectancy is held to be necessary for preparation behaviour to occur, the individual must also know what preparation actions to take and how to take them. Community Engagement Theory proposes that individuals consider the guidance and behavioural modelling of members of their community. In this way, individual beliefs interact with social factors (e.g., community participation) and relationships (e.g., trust). However, the social factors would be difficult to address in a broad, generalized intervention 
strategy such as the public education campaigns which this thesis intends to inform. The social factors within the CET, as suggested by the name, are more appropriate for approaches to target and engage with individual communities. Such research is important but beyond the scope and aims of this thesis.

Further, CET research encounters the same problem as PADM research whereby factors are selected from the full theory to test in a smaller analytical model, as some components are more or less useful in different contexts; for example, when a hazard is familiar, trust in external information sources is less important than when the hazard is unfamiliar (Paton, 2013). This method is useful in certain contexts, where the primary aim is to explain behaviour as fully as possible and therefore only using variables already established in the literature is beneficial. However, this method is less useful in contexts such as that of this thesis where one aim is to identify novel factors relating to a specific behaviour. Along these lines, the CET intends to explain behaviour but includes a number of broad categories of behaviour; this is useful in that it allows individual studies to operationalize behaviour in the way most appropriate for the research context, but limits the ability to compare findings across studies which use different operationalizations.

Instead, I preferred to use a theory which explicitly lays out a workable number of cognitions which can feasibly be manipulated to influence behaviour, including some which have already been supported in the natural hazard literature and some which have been supported in other behavioural domains and therefore can contribute new knowledge to the hazard literature.

\section{Theory of Planned Behaviour}

The above review of theories applied to natural hazard preparation is by no means extensive nor exhaustive. Other theories exist which have also been used in this domain but were not specifically designed for such applications (e.g., Vested Interest Theory and the Health Belief Model; see Ejeta et al., 2015 for a more detailed review). This section introduces the Theory of Planned Behaviour (TPB; Ajzen, 1991) as an appropriate theory to apply in this thesis. Importantly, I do not suggest that the TPB is the "best" theory but that out of the theories with a history of application in this research domain, the TPB is appropriate given the specifics of the context and the goals of this thesis.

Some researchers in the hazard domain argue for the benefits of an "all-hazards" model which incorporates the factors that have been shown to influence preparation for natural hazards (e.g., Paton, 2013). While such a model would have excellent conceptual utility, it would be difficult to measure all factors within a single set of studies. First, the range of factors 
influencing preparation behaviour is wide and diverse as demonstrated by the large number of theories and models adapted for hazard-related behaviours. Attempting to include all of these into a single model would reduce the predictive power of any single factor and make the model difficult to apply. Therefore, we should aim to find a model which can provide good predictive power, covering key variables without becoming too extensive. I propose that the TPB is a good candidate. Its current three components have been tested extensively and themselves comprise processes which have been identified to influence behaviour. Refining and developing these components should strengthen the TPB as a useful and reliable model while clarifying its relevance to natural hazards.

The TPB does not incorporate the threat perception which is critical to Protection Motivation Theory and the Protective Action Decision Model but does incorporate social and cognitive factors like Social Cognitive Theory. In contrast, however, the TPB specifies constructs within these broad categories including outcome expectancy, key to Community Engagement Theory, and self-efficacy, key to Social Cognitive Theory, Protection Motivation Theory, and to a lesser extent the Protective Action Decision Model. The TPB only includes factors which can be influenced to change behaviour, so is particularly useful for informing and evaluating an intervention.

The focus of the TPB also makes it appropriate for informing an intervention; the theory proposes a sufficient number of factors to give a high chance of identifying at least one which could be successfully targeted while not incorporating so many factors that key ones risk being obscured, or measures become exhaustively long. Finally, much previous research has focused on perceptions of the natural hazard itself, rather than perceptions of preparation behaviour even though previous research has demonstrated that the latter are more predictive of preparation for natural hazards than the former (Lindell, Arlikatti, \& Prater, 2009; Lindell \& Whitney, 2000). The reasons given above for the selection of the TPB over other key theories in the natural hazard literature are summarized in Table 2.1. Some evidence for the TPB meeting the criteria is presented in the following sections reviewing the theory. 
Table 2.1

Criteria used in theory selection.

\begin{tabular}{|c|c|c|c|c|c|}
\hline Criteria & PrE & PMT & PADM & CET & TPB \\
\hline Behaviour as specific outcome & No & Yes & Yes & Partially & Yes \\
\hline Not reliant on threat appraisal & No & No & Yes & Yes & Yes \\
\hline $\begin{array}{l}\text { All factors can be modelled } \\
\text { simultaneously }\end{array}$ & Yes & Yes & No & No & Yes \\
\hline $\begin{array}{l}\text { All factors targetable in an } \\
\text { intervention }\end{array}$ & Yes & No & No & No & Yes \\
\hline $\begin{array}{l}\text { Applied to a range of } \\
\text { behaviours/countries }\end{array}$ & No & Yes & Yes & Yes & Yes \\
\hline $\begin{array}{l}\text { Combination of supported and } \\
\text { novel factors }\end{array}$ & No & No & No & No & Yes \\
\hline
\end{tabular}

The TPB was developed from the Theory of Reasoned Action (TRA; Fishbein \& Ajzen, 1975), which proposes that the primary and immediate predictor of behaviour is intention to carry out that behaviour, with attitudes and perceptions of social norms about the behaviour explaining intentions. The TPB adds an additional component, perceived behavioural control (PBC), which is proposed to both have a direct effect on behaviour as well as the indirect effect through intentions. This latter model is now the more widely used, given consistent findings of the importance of $\mathrm{PBC}$, although findings around its direct and indirect influences are less consistent (Armitage \& Conner, 2001). The TPB therefore retains the proposal that the primary determinant of behaviour is intention to carry out that behaviour (see Figure 2.4). Intentions in turn are informed by attitudes about the behaviour and perception of social norms relating to the behaviour, as well as perceptions of control over carrying out the behaviour. These cognitions are in turn informed by beliefs, which are discussed in detail later. 


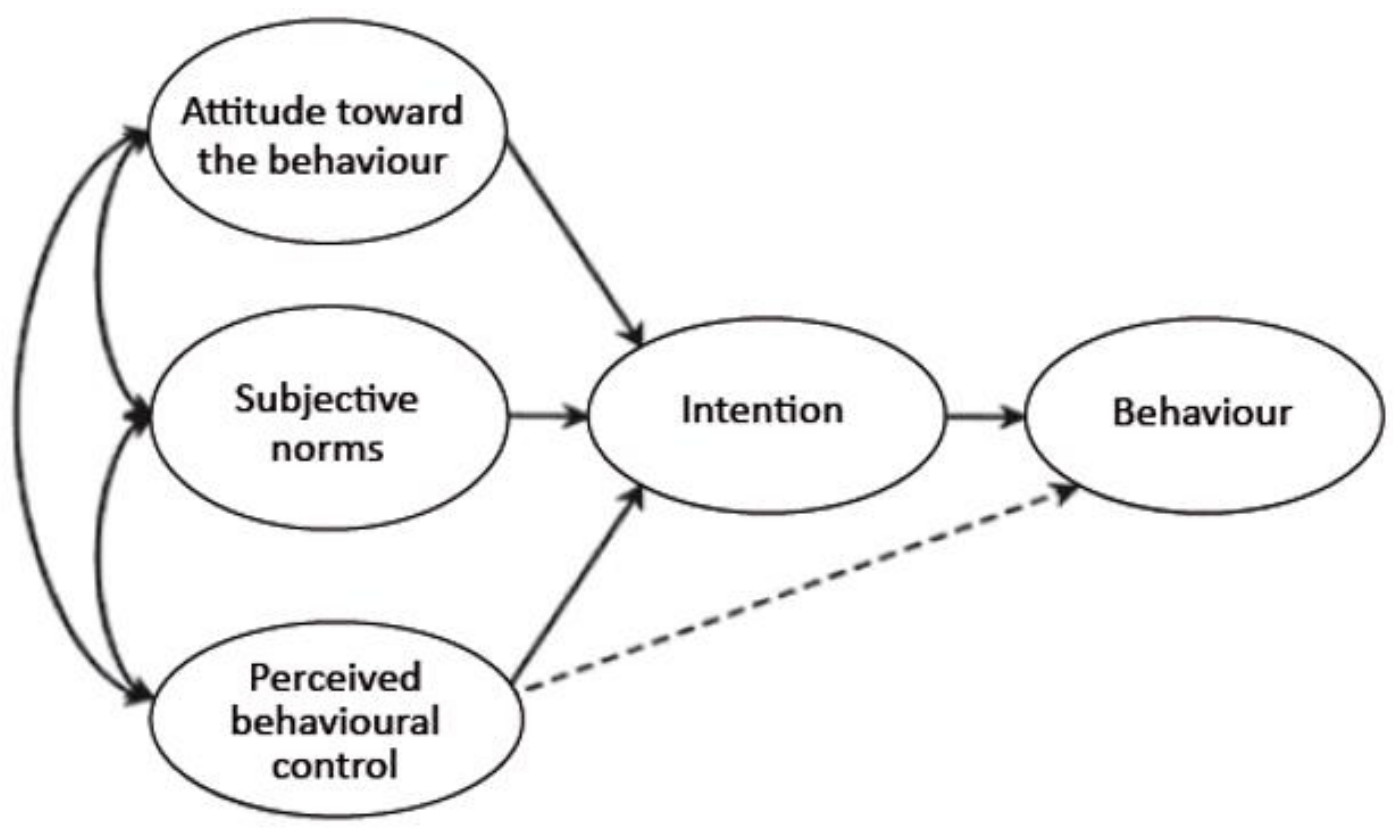

Figure 2.4. The original Theory of Planned Behaviour (Ajzen, 1991). The dashed line between perceived behaviour control and behaviour indicates the unconfirmed nature of this association.

\section{Intentions}

One of the key contributions of the TPB is its framing of intentions as the proximal predictor of behaviour. This factor is typically measured with questions regarding whether individuals plan, expect, want, or intend to engage in the behaviour (e.g., Francis et al., 2004). Intentions are particularly relevant for behaviours that require planning (hence Theory of Planned Behaviour) and conscious thought. Whether people intend to carry out that behaviour (e.g., preparing for a natural hazard) typically predicts between a fifth and half of the variance in behaviour (Hardeman et al., 2002; Webb \& Sheeran, 2006). Few other predictors of behaviour, with the exclusion of past behaviour, explain this much variance (Armitage \& Conner, 2001; Armitage, Reid, \& Spencer, 2013). The TPB model focuses on factors which explain intentions rather than factors which explain the remaining variance in behaviour not predicted by intentions. Some versions of the theory presented by Ajzen include PBC as a moderator of the intention-behaviour relationship or as a direct predictor of behaviour, though findings on this are inconsistent (McDermott et al., 2015) and are often weak (e.g., Guo, Wang, Liao, \& Huang, 2016). 
The so-called intention-behaviour gap (Sniehotta, Scholz, \& Schwarzer 2005) presents a challenge to increasing uptake of behaviour as interventions informed by the TPB target the predictors of intentions rather than directly targeting behaviour itself. While several factors not included in the TPB potentially affect the intention-behaviour relationship, such as length of time between measuring intentions and behaviour (Ajzen, 2012) and whether behaviour is objective or self-report, interventions that do not increase intentions show much smaller increases in behaviour (Webb \& Sheeran, 2006). The inclusion of intentions in the TPB therefore can improve the effectiveness of interventions; while the theory does not directly address the intention-behaviour gap it does at least empirically identify the issue unlike similar models. Several authors of TPB reviews and meta-analyses propose investigating mediators (McEachan, Conner, Taylor, \& Lawton, 2011) as well as testing implementation intentions and goal setting as possible additions to the theory to help address the intention-behaviour gap (e.g., Rhodes \& Dickau, 2012; Riebl et al., 2015). While these factors are not tested in this research, their potential in relation to natural hazard preparation is discussed later as avenues for future research.

\section{Attitudes}

Within the TPB, the first component proposed to explain behavioural intentions is attitudes about the behaviour; this refers to whether individuals evaluate the behaviour as positive or negative (Ajzen, 2011a). Much past research in the hazard domain focuses on aspects of the risk which relate to behaviour; however, it is also important and likely more useful in at least some contexts to consider perceptions of the behaviour of preparing (Lindell, Arlikatti, \& Prater, 2009). The attitudes component of the TPB, by intentional design, is focused on how people view the target behaviour rather than how they view the object of the behaviour (e.g., natural hazard risk) as this can be more predictive of behaviour (Lindell et al., 2009).

While Likert or Likert-type response scales can be used to measure attitudes, the construct is typically measured with semantic differential scales where one end of the scale is labelled with the antonym of the label at the other end (Ajzen, 2002). For example, if the aim is to understand why people engage in regular exercise, participants can indicate if they think that the behaviour is "Harmful" (1) to "Beneficial" (7)" (Ajzen, 2002). Scores closer to seven would represent a more positive attitude towards engaging in regular exercise, and those with more positive attitudes would be predicted to have stronger intentions to engage in regular exercise. 
The attitude component is differentiated into attitudes about the experience of enacting the behaviour (experiential attitudes) and attitudes about the effects of the behaviour (instrumental attitudes; Francis et al., 2004). The above exercise example aligns with instrumental attitudes as it refers to whether engaging in regular exercise will have the intended, positive effect of benefiting the person's health. Experiential attitude items refer to judgments such as if the behaviour is "Pleasant" or "Unpleasant" and "Enjoyable" or "Unenjoyable".

The attitude component is frequently found to significantly explain variance in intentions, with meta-analytic effect sizes for the association ranging from small ( $r=.16$; Topa $\&$ Moriano, 2010) to large ( $r=.61$; McDermott et al., 2015). The two subcomponents have also been found to uniquely relate to intentions (e.g., Ajzen, 2011b; Walker, Courneya, \& Deng, 2006; Wan, Shen, \& Choi, 2017) suggesting that the way people think about the outcome of a behaviour and the experience of enacting a behaviour are sufficiently discrete to be studied as separate constructs. However, both types of attitudes are not always used; studies which do not include the distinction typically default to measuring instrumental attitudes (Darker, French, Longdon, Morris, \& Eves, 2007).

There is considerable empirical support for the importance of instrumental attitudes to many behaviours, especially when considering similar constructs such as outcome expectancy and response-efficacy, as well as a long history of theorizing relating to attitudes focused on those regarding the outcome of the behaviour (Ajzen, 2012). Such support for the importance of experiential attitudes is far more limited. It is likely that instrumental attitudes are seen as more straightforward to manipulate than experiential attitudes; for example, there are likely fewer reasons why someone might not believe strengthening their home's foundations will reduce earthquake damage than why they might not believe having their foundations strengthened would be a positive experience.

\section{Social Norms}

Social norms relate to influences on an individual's behaviour from what is "normal" within that individual's social group. Importantly, there are two levels at which social norms exist; individual and societal (Rimal \& Lapinksi, 2015). Social norms are guides to behaviour which are generally shared within a social group but not formalized as laws and less stable (e.g., more context dependent) than traditions. Collective or actual social norms are the objective prevalence and strength of social norms in a group, which can be measured by assessing the behaviour of individual members of the social group. Perceived norms, by contrast, are considered at the individual level, whereby an individual develops a perception of the actual norms within their social groups. Communicating actual social norms can be an 
effective way to change behaviour; however, this effect is typically due to inaccurate perceptions of those social norms (Rimal \& Lapinksi, 2015). Therefore, it is important when aiming to understand behaviour to identify influences of perceived social norms, with communicating actual norms presenting one way of shifting norm perceptions and therefore, theoretically, behaviour. Because the TPB aims to identify predictors of behaviour, the norm component inherently, though often implicitly, measures perceived norms. As such, this thesis focuses on and measures only perceived norms.

Originally, the TPB included subjective norms, which are perceptions of pressure to behave in a certain way. This type of norm has been implicitly and explicitly equated with injunctive social norms, which refer to whether a behaviour is approved of or not within a social group (e.g., Ajzen, 2002; White et al., 2009). In contrast, descriptive social norms refer to the prevalence of the behaviour within a social group (Cialdini, Reno, \& Kallgren, 1990). Many studies still use the concept of subjective norms (e.g., Elliott \& Armitage, 2009) but often conflate the concept with that of injunctive norms. For example, researchers typically test subjective norms by asking whether participants think that a number of different referents, such as friends, family, or colleagues, approve of the behaviour (e.g., Sun, Acheampong, Lin, \& Pun, 2015; Zhu, Zhang, Ling, \& Wan, 2017), which closely represents an injunctive norm. This mixing of subjective norms with perceived injunctive norms persists within theoretical discussions of the concepts (Farrow, Grolleau, \& Ibanez, 2017; White et al., 2009), with the use of subjective norms still prevalent in the TPB literature (e.g., Najafi et al., 2017; Sun et al. 2015; Zhu, Zhang, Ling, \& Wan, 2017).

However, the distinction between descriptive and injunctive norms is becoming more common (e.g., Lake, Milfont, \& Gavin, 2011; Walker, Courneya, \& Deng, 2006) in line with recommendations from the theory creator (e.g., Ajzen, 2002; Ajzen, 2007) and consistent with current understanding in social norms theory (e.g., Cialdini et al., 1990; Park \& Smith, 2007, Vinnell et al., 2018). Previous research has repeatedly demonstrated that descriptive and injunctive norms are discrete concepts (e.g., Vinnell et al., 2018) with different motivational mechanisms (Hamann, Reese, Seewald, \& Loeschinger, 2015). Descriptive norms, conveying how common a behaviour is, provides an individual with information about whether a behaviour is likely to be beneficial; that is, if many similar others are engaging in a behaviour, it is likely because that behaviour is useful. In contrast, injunctive norms motivate behaviour as they provide a way for individuals to avoid social punishment for engaging in a behaviour which is not approved or gain social reward by engaging in an approved behaviour. 
While this distinction is perhaps the most frequently used within the TPB literature, much current research still does not apply it. The norm component of the TPB is often found to have the weakest association with intentions out of the three TPB components (e.g., Armitage \& Conner, 2001, Chan \& Tsang, 2011; Guo, Berkshire, Fulton, \& Hermanson, 2017). However, this is possibly due to the prevalence of subjective norms rather than the more appropriate distinction between descriptive and injunctive norms (Armitage \& Conner, 2001).

Ajzen's discussion of, and recommendations for, the norm component are also conflicting, perhaps contributing to inconsistencies in the literature. For example, his 2013 questionnaire guide includes only subjective norms, missing the descriptive component which he recommended in 2002 (Ajzen, 2002; Ajzen, 2013). As this thesis intends to create a comprehensive set of measures for the TPB, it uses the distinction between descriptive and injunctive norms which is consistently advocated and specified in norms theory and recognized within the TPB literature. For the sake of brevity, references throughout to "descriptive norms" should be read as "perceived descriptive social norms" and "injunctive norms" as "perceived injunctive social norms", unless otherwise stated.

\section{Perceived Behavioural Control}

Finally, PBC refers to the level of control that individuals perceive themselves to have over whether they carry out the behaviour. This component was added to the TRA to form the TPB in recognition that a behaviour must be at least partially volitional for an individual to form an intention to undertake it. Ajzen (2012) argues that for the TPB to be relevant to a behaviour, the given behaviour must be one over which the participant has some control; that is, the participant has to be able to choose to carry out the behaviour. However, the behaviour cannot be entirely volitional. If a participant believes that carrying out the behaviour is entirely based on whether they choose to do it or not, and thus does not perceive any other barriers, the TPB will not predict behaviour.

Like attitudes, PBC is typically treated as a single construct, although Ajzen (2002) proposed two separate factors. Specifically, PBC can refer to whether individuals are able to make a decision about carrying out the behaviour, rather than the behaviour being seen as involuntary (controllability), as well as whether individuals have the capability to carry out the behaviour in terms of individual factors such as time, knowledge, effort, and finances (selfefficacy). The two PBC factors of controllability and self-efficacy are currently the least clearly defined in the TPB literature. Many studies before the introduction of the distinction by Ajzen (2002) used self-efficacy as a proxy for PBC (Hardeman et al., 2002), though this conflation of self-efficacy with PBC has continued since (Armitage, Reid, \& Spencer, 2013) with few 
exceptions (such as Lake et al., 2011). Self-efficacy is usually more important for explaining intentions, although controllability has been shown to predict behaviour when self-efficacy did not (Ajzen, 2002).

Unlike the distinction in attitudes between instrumental and experiential, which is clear conceptually with some empirical support, and the distinction between descriptive and injunctive norms, which is clear conceptually with thorough empirical support, the distinction between self-efficacy and controllability is not well developed. This is despite being included in some TPB questionnaires over a decade ago (e.g., Ajzen, 2002). In this questionnaire, Ajzen states that self-efficacy refers to "difficulty of performing the behavior, or ... the likelihood that the participant could do it" while controllability refers to whether individuals perceive they "have control over the behavior, that its performance is or is not up to them" (Ajzen, 2002, p. 7). While this distinction is in some ways clear, for example that an individual's perception of whether they can choose to engage in a behaviour is not the same as their perception of whether they can carry out that behaviour, there is also a lack of clarity; an individual might believe they cannot choose to do something because they do not believe they would be able to do it, and in this way interpret a question about whether it is "up to them" to carry out a behaviour in a similar way as they would a question about whether they could carry out that behaviour. It is possible that this lack of clarity is exacerbated by a lack of clarity in the English language; saying one could do something has both a permissive meaning (i.e., they are allowed to do it) and a practical meaning (i.e., they are capable of doing it) which reflect controllability and self-efficacy, respectively.

\section{Beliefs}

The three TPB constructs of attitude, norms, and PBC outlined above are said to be direct; that is, scale items are intended to measure the actual constructs and the constructs directly explain intentions. The full version of the TPB also includes beliefs which determine the "direct" components and so are said to be "indirect", measuring different aspects of the constructs (Ajzen, 2002). Ajzen (2006a) proposed these beliefs as the antecedents of the TPB constructs (e.g., attitude beliefs precede attitudes), although he also argued that belief-based measures are assessing the same latent construct as "direct" measures and should not be treated as antecedents of the components explaining intentions (Ajzen, 2002). This research will treat beliefs as antecedents based on evidence of beliefs preceding the cognition components (McMillan et al., 2009).

For clarity, the three TPB components reviewed above (attitudes, norms, and PBC) will be referred to as "cognitions" to differentiate them from the preceding beliefs. People develop 
beliefs about a behaviour, which then influence the attitudes, perceptions of norms, and perceptions of control that they develop. If these beliefs operate as suggested, then targeting these beliefs, rather than the constructs they inform, could lead to greater success in interventions (Ajzen, 2006a; Darker et al. 2007). This strategy of targeting beliefs has some support; for example, Elliott and Armitage (2009) successfully increased road safety behaviour by increasing control beliefs, which in turn increased PBC.

Each belief includes two components that are multiplied to create a belief score. Attitude beliefs comprise behaviour belief strength and outcome evaluation. The former refers to how strongly the person endorses the behavioural belief while the latter refers to whether the person thinks a particular outcome of the behaviour is likely to be positive or negative. Both descriptive and injunctive norm beliefs incorporate normative belief strength; for example, a direct descriptive norm item might ask about perceptions of behaviour generally while a normative belief strength item will ask about perceptions of behaviour among a specific referent group, such as the participant's family. Descriptive norm beliefs also include how strongly the participant identifies with the particular referent groups while injunctive norm beliefs include how motivated the participant is to comply with what those referent groups think they should do. Finally, PBC belief measures include control belief strength and power of control factors. The first refers to how likely the participant thinks it is that they will face the particular barriers to preparing presented in the direct measure while the latter refers to how much influence participants think that barrier will have on their behaviour.

Belief measures are seldom included in TPB studies (e.g., Hardeman et al., 2002) which potentially explains why distinct measures of the belief factors have not been developed in line with the distinctions introduced in the cognition components; for example, attitude belief measures are not specific for experiential or instrumental attitudes. These issues are discussed below in the section summarizing the methodological weaknesses in the current TPB literature.

\section{Support for the TPB}

Armitage and Conner (2001) conducted a meta-analytical summary of 185 studies that have used the TPB to explain a variety of behaviours, from playing basketball to car use. Their meta-analysis provided overall support for applying the theory, with an average of $39 \%$ of variance in intentions explained, while also identifying weaknesses to address, such as poor operationalization and inconsistent measurement of the subjective norm component. Since then, other meta-analyses have found support for the usefulness of the TPB in predicting several different types of behaviours, including smoking (Topa \& Moriano, 2010), breastfeeding (Guo et al., 2016), and healthy eating (McDermott et al., 2015; Riebl et al., 2015). 
Fewer studies, and therefore fewer meta-analyses, have examined the TPB in regard to behaviour change interventions. For example, Riebl et al. (2015) found 34 articles that applied the TPB to youth dietary behaviours, which included only three interventions. In their review, Hardeman et al. (2002) found that most intervention studies used the TPB to measure change or predict behaviour change, rather than to inform the strategies employed in the intervention. However, simulations suggest that interventions targeted to TPB components would typically lead to an approximate $28 \%$ increase in the proportion of the sample carrying out the behaviour (Fife-Schaw, Sheeran, \& Norman, 2007).

Consistent with findings around prediction, Webb and Sheeran (2006) meta-analysed 47 studies which experimentally manipulated intentions, using a range of theories including the TPB, and found a medium to large effect on intentions and a small to medium effect on behaviour. Tyson, Covey, and Rosenthal's (2014) review of intervention studies to reduce heterosexual risk behaviours using the TPB (or TRA) found a similar small effect on all components, including behaviour. These meta-analyses and reviews support the TPB as a prediction and measurement tool, as well as providing some support for the theory as a way to inform behaviour change interventions. However, there are several ways in which the typical application of the theory can be improved. The majority of these ways have already been suggested within the literature and mentioned above but are not consistently addressed.

\section{Weaknesses in TPB Research}

As well as extending the application of the TPB to a new domain, the present research affords the opportunity to develop the model. There are three main ways in which this thesis aims to address some of the more major limitations raised within the literature. First is what I will term the two-factor distinction; that is, incorporating the theorized factors of the cognition components (e.g., experiential and instrumental types of attitudes). Second, I will avoid inconsistencies in measurement and modelling, such as inconsistent inclusion of beliefs by testing the full TPB model including all proposed factors. Finally, issues around conflicting operationalization of the behaviour component of the theory will be raised and addressed to the extent possible within a single research project.

The two-factor distinction. As has already been mentioned above, various research as well as theorizing within the literature has proposed two distinct factors for each of attitudes, norms, and PBC. Although attitudes have been recognized as comprising both instrumental and experiential (or affective) components for over a decade, few studies include this distinction. Indeed, Darker et al. (2007) report that the predictive capability of the two types of attitudes differ but argue that more evidence is necessary. 
Further, some researchers have argued that the subjective norm component should be dropped given weak and inconsistent findings in the literature, while others have argued that the weakness in this element could be due to poor and inconsistent operationalization and measurement, which often includes single-item measures (Armitage \& Conner, 2001). Since the development of the model, Cialdini et al.'s (1990) focus theory of normative conduct has proposed a clearer definition of norms which divides them into two categories: descriptive and injunctive. Part of the inconsistency again could be due to Ajzen's inconsistent recommendations about his model. In a 2011 book chapter, he presents the norm component as exclusively comprising subjective norms (Ajzen, 2011a). However, he has also argued has argued for the inclusion of this differentiation (Ajzen, 2006a, 2011b). Applying this differentiation to the TPB norm component in the present research can therefore provide firmer support for its continued inclusion in the model.

Establishing whether inconsistencies and lack of refinement in normative measures leads to the weakness in the norm component will help to strengthen the model. The possibility exists, however, that the weakness is not entirely explained by these factors. Norms act as a mental shortcut when deciding how to behave (Goldstein, Griskevicius, \& Cialdini, 2007), typically fast (Farrow, Grolleau, \& Ibanez, 2017) and below the level of awareness (Nolan et al., 2008). With the importance the TPB places on the formation of intentions, it likely models a more intensive cognitive process. It is possible that the influence of perceived norms is weaker on the types of behaviour which require intention formation, as opposed to behaviours which are more spontaneous such as littering (Cialdini et al., 1990).

Finally, authors of meta-analyses and reviews have also disputed the operationalization of PBC (e.g., Armitage \& Conner, 2001). Previous research has found that both PBC and selfefficacy play a significant role in the model, although they were not historically tested in conjunction (see Armitage \& Conner, 2001 for a review). In fact, some uses of the model define PBC as self-efficacy (Ajzen, 2011a). As detailed before, recent research suggests that PBC has two distinct but related sub-components: perceived controllability (whether the individual thinks they have control over carrying out the behaviour) and self-efficacy (whether the individual thinks they are able to carry out the behaviour; Ajzen, 2002). Therefore, this construct requires further testing and potentially more clarification.

Measurement and modelling. Several authors have argued that an explanation for conflicting results in research applying the TPB is the use of different measures (e.g., Armitage \& Conner, 2001). First, studies often create TPB measurement items within the context of the individual study, rather than adapting consistently used and therefore validated scales. Second, 
there is often inconsistency in the inclusion and operationalization of the constructs themselves. As described in the previous section, the TPB has been developed considerably since it was first introduced, with possible two-factor structures proposed for each of the three cognition components (i.e., attitudes: experiential and instrumental; norms: descriptive and injunctive; and PBC: controllability and self-efficacy).

Finally, a further inconsistency in applications of the TPB lies in the inconsistent inclusion of indirect measures of the beliefs theorized to precede the three TPB components. Again, these beliefs have been included in theorizing of the model for many years (e.g., Ajzen, 2006a; Francis et al., 2004), and yet are seldom tested, and even less frequently tested thoroughly. Some studies treat beliefs as indistinct from the constructs themselves (e.g., Sun et al., 2015) and across studies beliefs are not measured using consistent methodology. Including these beliefs in the present research, therefore, represents an important contribution to the literature, regardless of findings. This thesis goes one step further and in Study 3 tests whether the two-factor distinction within the cognitions should be applied to beliefs. For example, whether the model is improved by including specific constructs of experiential attitude beliefs and instrumental attitude beliefs, rather than the same attitude belief construct for both types of attitudes.

Similarly, the analysis used to test the TPB varies between studies. Many use regression analysis (Topa \& Moriano, 2010) while others use structural equation modelling (SEM) as recommended by Ajzen (2011a). SEM analysis is usually preferred as it deals better with error and variances within data; however, findings can differ between a stepwise regression analysis and a path analysis such as SEM where all paths are tested simultaneously (Topa \& Moriano, 2010). Finally, the studies which do test a possible direct effect of PBC on behaviour produce inconsistent findings (Armitage \& Conner, 2001). It is possible that this effect of PBC varies depending on the behavioural context. It is also possible that one PBC subcomponent relates more to behaviour than the other and therefore findings might differ depending on how PBC has been operationalized within the individual studies (Ajzen, 2002).

Behaviour. Regardless of weaknesses in the individual components of the model, one of the main challenges of applying the TPB is the definition and measurement of behaviour. Given the wide number of behaviours to which the theory has been applied, there are several inconsistencies in measurement of the behaviour under study. First, some studies refer to specific behaviours (such as having a blood pressure test; Francis et al., 2004) while others refer to general classes of behaviour (such as eating healthy or exercising, which involve several different behaviours; Riebl et al., 2015). Ajzen (2011a) argues that the TPB should 
function similarly for both specific behaviours and categories of behaviours, so long as the predictor and behaviour measures are compatible. Previous studies demonstrate strong prediction of broad categories of behaviour as will be used in this thesis (Manning \& Bettencourt, 2011).

Second, and in line with the lack of an agreed-upon and uniformly applied set of measures, there is a large amount of variation within studies looking at substantively the same behaviours as to how those behaviours are worded. For example, Chan and Tsang (2011) asked their participants about "healthy eating", Hewitt and Stephens (2007) asked about specific healthy eating behaviours such as "eat some fruit", and Armitage and Conner (1999) asked about eating "a low-fat diet", while other studies ask about harmful eating behaviour such as consuming sugary snacks (e.g., Åstr®m \& Okullo, 2004). Positive versus negative valence framing can impact behaviour (e.g., Block \& Keller, 1995; Vinnell, McClure, \& Milfont, 2017; Yang, 2013), and beyond introducing potential framing effects, the inconsistency in how the outcome measures are framed introduces the issues of general versus specific behaviours as well as positive versus negative behaviours, making it difficult if not inappropriate to compare findings across studies. Further, previous research has demonstrated that intentions to do something (i.e., engage in positive behaviour) differ from intentions to not do something (i.e., abstain from negative behaviours; Richetin, Conner, \& Perugini, 2011), including in relation to preparing for natural hazards (Paton, Smith, \& Johnston, 2005). This difference in positive and negative behaviours could explain some of the inconsistency in how well the TPB explains intentions and predicts behaviour. This thesis does not explore the role of valence framing in this way but recognizes it as an important avenue for future research. The effects of valence framing are likely important to consider when developing measures of intentions and behaviour and when comparing studies which use differently worded measures.

Further, TPB studies tend to use self-report measures of behaviour (e.g., Hardeman et al., 2002) which are open to biases such as social desirability (van de Mortel, 2008) as such data are typically easier, quicker, and cheaper to collect (Ajzen, 2002). Ajzen (2002) suggests that one way to improve the validity of self-report behaviour measures is to use multiple items and that the accuracy of self-report measures differs between contexts (Ajzen \& Fishbein, 2004). It is likely that individuals will be better at accurately reporting whether they have stored water than how many times in the past month they have eaten fruit, for example. Research on how behavioural measurement affects the intention-behaviour relationship is conflicted, with some showing a stronger association for self-report measures (Armitage \& Conner, 2001; 
McEachan et al., 2011) and some showing a stronger association for objective measures (Webb \& Sheeran, 2006).

Another main challenge of applying the TPB is the intention-behaviour gap (Sniehotta et al., 2005). Given consistent findings that intention does not always lead to behaviour (Armitage \& Conner, 2001), this research will also test factors relating to preparation, in line with the suggestions of Ejeta and colleagues (2015). Such factors include ethnicity, which has been shown to influence social norm effects (e.g., Fischer, Karl, \& Fischer, 2019; Heinrichs et al., 2006), age, gender, education, and income (Ejeta et al., 2015; Lindell \& Whitney, 2000). Other factors which have been shown inconsistently to affect natural hazard preparedness, such as previous experiences in disasters (Becker, Paton, Johnston, Ronan, \& McClure, 2017; Bourque et al., 2012; Lindell, 2013) and risk perception (Becker, Paton, \& Johnston, 2015; Bourque, 2013; Bourque et al., 2012; Perry \& Lindell, 2008; Solberg et al., 2010) will also be considered.

\section{The TPB in Natural Hazard Literature}

Few studies have explicitly applied the TPB to natural hazard preparation; these studies are reviewed at the end of this section. However, several of the components within the TPB have either been directly studied in relation to natural hazards or are similar to other constructs studied in the field. Local and international literature demonstrating the relevance of the individual TPB components to natural hazard preparation is therefore presented below to add support for the application of the theory in this research.

Attitudes in natural hazard literature. Instrumental attitudes have several equivalents within the natural hazard literature; commonly, the terms response-efficacy or outcome expectancy are used (Becker et al., 2015). These concepts refer to the perception that the behaviour will be effective at creating the expected outcome; that is, the perception that preparing for a natural hazard will increase the chances of survival, reduce the amount of damage experienced, and improve the recovery process. Much research supports the importance of these constructs to disaster preparation (Becker et al., 2015; Johnston et al., 2013; Lindell \& Whitney, 2000; Paton et al., 2005). For example, response-efficacy showed the strongest association with support for public legislation to strengthen earthquake-prone buildings in Wellington out of all tested factors (Vinnell et al., 2017). Moreover, those who hold negative outcome expectancies, also termed fatalism (i.e., the belief that preparation actions will not help in the event of a disaster), have been shown to be less likely to prepare for natural hazards than those with a positive outcome expectancy (e.g., Paton et al., 2010; Terpstra \& Lindell, 2012). Experiential attitudes do not have a close proxy in the existing disaster risk 
reduction literature so any findings showing the relevance of this factor to natural hazard preparation would represent a potential avenue for further research and intervention.

Social norms in natural hazard literature. Some research (e.g., Sanquini, Thapaliya, \& Wood, 2016) has examined the role of social cues and observation of behaviour on earthquake preparation without specifically examining social norms. An interview study with New Zealand participants identified a perceived norm of "unpreparedness", despite a shared belief that preparing is a positive behaviour (Becker, Paton, Johnston, \& Ronan, 2014). In this interview study, those who were prepared reported that they were put off from encouraging others to do the same because of potential social punishment for contravening the descriptive social norm (e.g., being perceived as "weird"). McIvor and Paton (2007) found that subjective norms related to intentions to prepare for earthquakes, although this relationship operated indirectly through perceptions of response-efficacy (whether the behaviour will have the intended effect). Whether an individual observes others preparing can explain that individual's preparedness (Bourque, 2013) while research in Japan used descriptive norm information to influence food storage behaviour as a preparation action for earthquakes (Ozaki \& Nakayachi, 2015). However, this finding should be replicated and extended to include injunctive norms as well as to address a wider range of preparation behaviours and natural hazards.

Social norms have also been used experimentally to influence judgments about earthquake-strengthening legislation in Wellington (Vinnell et al., 2018). This legislation requires that buildings deemed to be earthquake-prone have to be strengthened above a certain threshold in a timeframe of approximately 15 years. A message which presented the rate at which buildings are being strengthened (i.e., a descriptive social norm) led to participants regarding the total work required by the legislation as more feasible to achieve within the timeframe. Furthermore, a message which presented the percentage of people who approved of the legislation (i.e., an injunctive social norm) led to stronger support for the legislation. This experimental research demonstrates that social norms can be used to change judgments about earthquake-related preparation. However, the potential for social norms to change behaviour comprising natural hazard preparation both requires and merits investigation (Vinnell et al., 2018; Vinnell, Milfont, \& McClure, 2019).

Perceived behavioural control in natural hazard literature. Unlike instrumental attitudes, self-efficacy as used in the TPB is identical to self-efficacy as commonly used in the natural hazard literature. Self-efficacy is an important factor in people's actions to prepare themselves for a natural hazard (Becker et al., 2015; Duval \& Mulilis, 1999; Levac et al., 2012; Lindell \& Whitney, 2000; Paton et al., 2005; Rowney, Farvid, \& Sibley, 2014). For example, 
Levac and colleagues (2012) found that self-efficacy was positively related with household preparedness for emergencies (the term used by the authors). Evidence from the 2010-11 Canterbury earthquake sequence demonstrates that the relationship between self-efficacy and behaviour is reciprocal; taking preparation action can increase self-efficacy, with an added benefit of protecting against psychological stress (Rowney et al., 2014), while higher selfefficacy leads to more preparation. Therefore, increasing perceptions of self-efficacy to prepare for natural hazards can increase preparation behaviour, which will then further increase selfefficacy, leading to a self-maintaining system where cognition and action beneficially impact each other.

As with experiential attitudes, the concept of controllability does not have a direct equivalent in the natural hazard literature. There are similarities to the concept of personal responsibility which has been demonstrated as relevant to natural hazard preparedness (Becker et al., 2015; Paton et al., 2005). The opposite of personal responsibility, the belief that others, typically local or national governments, have the responsibility to prepare, is negatively associated with preparedness (e.g., Paton, et al., 2005). This belief, however, is not identical to a lack of controllability, as an individual not having control does not necessarily imply that another entity does have control. As with experiential attitudes, controllability is included in the current research, both to be consistent with the theory and because significant findings for this factor would contribute another predictor of natural hazard preparation which could be useful to future research.

The full theory in natural hazard literature. In relation to hazards broadly, the full TPB has been used in studies of health preparedness, such as donating blood during different risk stages of an outbreak of bird flu, intentions to get an influenza vaccine, and volunteering of nurses for patients with SARS (see Ejeta et al., 2015 for a review). However, the TPB has not yet been thoroughly applied to the area of natural hazard preparation despite the decades of research supporting its utility in predicting and understanding behaviour change (e.g., Armitage \& Conner, 2001; McDermott et al., 2015; Riebl et al., 2015; Topa \& Moriano, 2010; Tyson et al., 2014) and support from researchers in the field of natural hazards (e.g., Beatson \& McLennan, 2011).

Beatson and McLennan (2011) discussed the applicability of the TPB to bushfire preparation in Australia. Their discussion includes an acknowledgement of a "supplemental" descriptive norm in addition to subjective norms and a potential direct effect of $\mathrm{PBC}$ on behaviour as well as explanations of the belief components. The authors argue that the current TPB theory has the potential to be usefully applied to intentions and behaviour related to 
preparing for bushfires. However, they also argue that the theory can benefit from expansion to include other important predictors. For example, Beatson and McLennan summarize the findings of a meta-analysis exploring the role of affect in the TPB, which found that including anticipated affect led to a $5 \%$ increase in the variance of intentions explained (see Rivis, Sheeran, \& Armitage, 2009). That is, how people imagine they will feel after carrying out the behaviour might influence behaviour (this meta-analysis did not examine the intentionbehaviour association). This exploration was based on the finding that the TPB is less predictive for health-related behaviours which entail strong affect. Although many potential additions to the theory can be proposed based on literature examining behaviours in other domains, no study has applied the full TPB to natural hazard preparation; doing so therefore is a logical next step before introducing new components.

Other researchers in Australia have since applied the TPB to understanding bushfire preparedness (Morrison, Lawrence, \& Oehmen, 2014). The model used in this research included belief components and a specific attitude scale similar to instrumental attitudes, although retained subjective norms and PBC. Both subjective norms and PBC, as well as some types of attitudes, related to preparedness. This research, therefore, shows that the TPB can be successfully applied to behaviour related to natural hazards. However, several improvements will be made in this thesis. First, the measures used by Morrison et al. (2014) were adapted from a large number of sources and each component scale included a different number of items. This thesis develops a questionnaire with consistent numbers of items across measures and sourced from as few places as possible. Second, Morrison et al. used the TPB components to explain a small amount of variance in current preparedness levels: attitudes and norms are only theorized to explain intentions, while PBC is also primarily, though not exclusively, used to explain intentions. This thesis uses the TPB to explain intentions to prepare as well as to predict preparation by measuring behaviour at a second time point.

Another study used the TPB to explain intentions to prepare for wildfires in the United States (Bates, Quick, \& Kloss, 2009). This study used attitudes, subjective norms, and PBC to explain intentions to mitigate wildfire risk. The PBC component measured the participants' perceptions of both their ability to carry out the actions and the efficacy of mitigation. Attitudes, subjective norms, and PBC all significantly and positively correlated with intentions to mitigate wildfire risk to one's home and together explained $51 \%$ of the variance in intentions, with PBC showing the strongest association.

In research which the authors claim is the first to apply the TPB to disaster preparation, Najafi et al. (2017) used the theory to explain intentions to prepare in Iran. The authors used a 
set of seven survival actions and split participants into "prepared" (10\% of sample; those who had undertaken at least five out of the seven actions presented in the survey) and "unprepared" (90\%; of this sample, $43 \%$ reported almost never preparing). Attitudes, subjective norms, and PBC all positively correlated with intentions, explaining between a third and a half of the variance in intentions, with attitude showing the strongest association. Moreover, those who had prepared had more positive attitudes, perceived more social pressure, had higher PBC, and stronger intentions than those who had not prepared. Najafi et al. (2017) used the self-reports of past behaviour both to split participants into "prepared" and "unprepared" categories and as the behaviour outcome in their structural model. Researchers in this field acknowledge that past behaviour is an important predictor of future behaviour (Armitage \& Conner, 2001) but other methods could improve on this treatment of past behaviour as a proxy for behaviour, such as the methods used in Studies 2 and 3 (Chapters 4 and 5).

While the TPB typically explains approximately $40 \%$ of the variance in behavioural intentions, the ability of the model to predict behaviour tends to be weaker (e.g., Armitage \& Conner, 2001). Najafi et al. (2017) found the same pattern for "unprepared" participants, with amount of variance explained dropping from $56 \%$ for intentions to $10 \%$ for behaviour. However, a much larger proportion of variance in behaviour was explained for "prepared" participants $(62.8 \%)$ compared to intentions for the same group (32\%). The authors suggested that this might be due to people not having a realistic perception of behavioural control and that reducing perceptions of difficulties might be a way to target behaviour. Alternatively, it might be that the TPB is better at predicting why people carry out a behaviour, rather than why they do not (Paton, Smith, \& Johnston, 2005; Richetin et al., 2011).

A later study in Iran, which authors claim is the first application of the TPB to earthquake preparation, explored salient beliefs related to household earthquake preparation (Najafi, Khankeh, Elmi, \& Pourvakhshoori, 2018). Using semi-structured interviews, these authors identified common beliefs that preparing can reduce injuries and deaths and can, negatively, lead to anxiety. Further, just under half of participants reported the belief that their family do not disapprove of preparing for earthquakes while the vast majority believe that education about how to prepare gives them more control over preparing. This study demonstrates the usefulness of the TPB to identify specific beliefs about preparing which can be targeted in public education campaigns. However, as the majority of participants (80\%) had not prepared, the authors could not test whether these beliefs actually related to preparation. Therefore, this study supports the application of the TPB in this thesis but allows for several methodological extensions. 
Kahlor, Olson, Markman, and Wang (2018) explored intentions to seek information regarding the relatively novel risk of induced earthquakes in Texas using the Planned Risk Information Seeking Model (PRISM; Kahlor, 2010) which incorporates the TPB factors of attitudes, subjective norms, PBC, and intentions with specific risk-related factors from the Risk Information Seeking and Processing model (RISP; Griffin, Dunwoody, \& Neuwirth, 1999). The authors distinguished descriptive and injunctive norms, although a different number of items were used to measure each (two versus six) and they were not included as separate predictors in the model. Kahlor et al. (2018) found that, of the TPB variables, attitudes and subjective norms were both significantly and positively associated with intentions - attitudes weakly and norms strongly — and together contributed the majority of the $70 \%$ of explained variance in intentions to avoid information about earthquake risk (with affect and perceived knowledge insufficiency also significantly associated with intentions).

The limited research above applying the TPB to natural hazard preparation, as well as the research presented for the individual TPB components, supports the application of the TPB to predict natural hazard preparation but also reinforces the necessity to refine the model. The reviewed literature provides more possibilities for refinement than can feasibly be included within a single study or set of studies as presented here. Therefore, the refinements to the theory undertaken in this thesis will focus on common and well-identified weaknesses pointed out above.

\section{Aims of the Thesis and Overview of Studies}

The practical aim of this thesis is to identify cognitive predictors of natural hazard preparation which can feasibly be targeted in broad public education campaigns; the TPB was selected as the most appropriate theoretical framework for this purpose. The theoretical aim of the thesis is to contribute to the understanding and implementation of the TPB both to this research domain and to studies of other behaviours. As described above, there are many inconsistences in how the theory has been applied and how its components have been measured. Therefore, this thesis aims to test a set of scales to measure all theorized components of the theory, including the distinctions between experiential and instrumental attitudes, descriptive and injunctive norms, and controllability and self-efficacy, as well as to measure the indirect, antecedent beliefs. These scales are based primarily on three TPB questionnaire development guides, use a consistent number of items, and undergo validation throughout the empirical studies. While one issue with consistent measurement is the variation in behaviours to which the TPB is applied, and so therefore the wording of items, the aim here is to produce 
measures which can easily be adapted, introducing minimal change, to different behaviours. The thesis comprises four studies detailed below.

Study 1. While much previous research demonstrates that preparation levels in Wellington are typically low, the first study of this thesis aimed to check and confirm these findings. An important preliminary step of designing a behaviour change intervention is to identify behaviours to target; these behaviours need to have the potential to lead to the desired outcome if increased (i.e., a particular behaviour to prepare for natural hazards to be targeted should be likely to improve outcomes in an event; McKenzie-Mohr, 2011). Further, the behaviour should not already be commonly done; targeting a behaviour which is already being carried out by many people will have less impact on the desired outcome than would targeting a behaviour which is less common. Study 1 also identified barriers to behaviour. Following the study of McClure, Spittal, Fischer, and Charleson (2015), this study asked participants to provide a reason for why they have not carried out each behaviour. While there are limitations to such self-report methods, this will help to inform any intervention strategies used to increase preparation.

As there have been few studies directly applying the TPB to natural hazard preparation, the second aim of Study 1 was to provide a preliminary test of the applicability of the theory to this behaviour using traditional measures of the original components. However, given the strong evidence from previous research for the distinction of descriptive and injunctive norms, Study 1 also measured these two discrete constructs. Further, a measure of self-efficacy was included to test for overlap between this construct and PBC and provide preliminary evidence for introducing this second distinction. This study offers justification and support for thoroughly developing and refining a questionnaire based on this theory for application in the current research context, as is recommended by the creator of the theory.

Study 2. Based on significant associations between TPB cognition components and intentions to prepare, Study 2 developed and provided a first test of a thorough and refined set of scales to measure the full TPB, including all two-factor distinctions as well as the belief components (see Figure 2.5). In line with the recommendations of Ajzen (2011a) and researchers of natural hazard preparedness (Ejeta et al., 2015; McIvor \& Paton, 2007), the data for this study were analysed using structural equation modelling (SEM) rather than more common regression analyses (Topa \& Moriano, 2010) which do not account for error to the same extent. Patterns of results can differ between stepwise regression and path analyses such as SEM where all paths were tested simultaneously (Topa \& Moriano, 2010). 


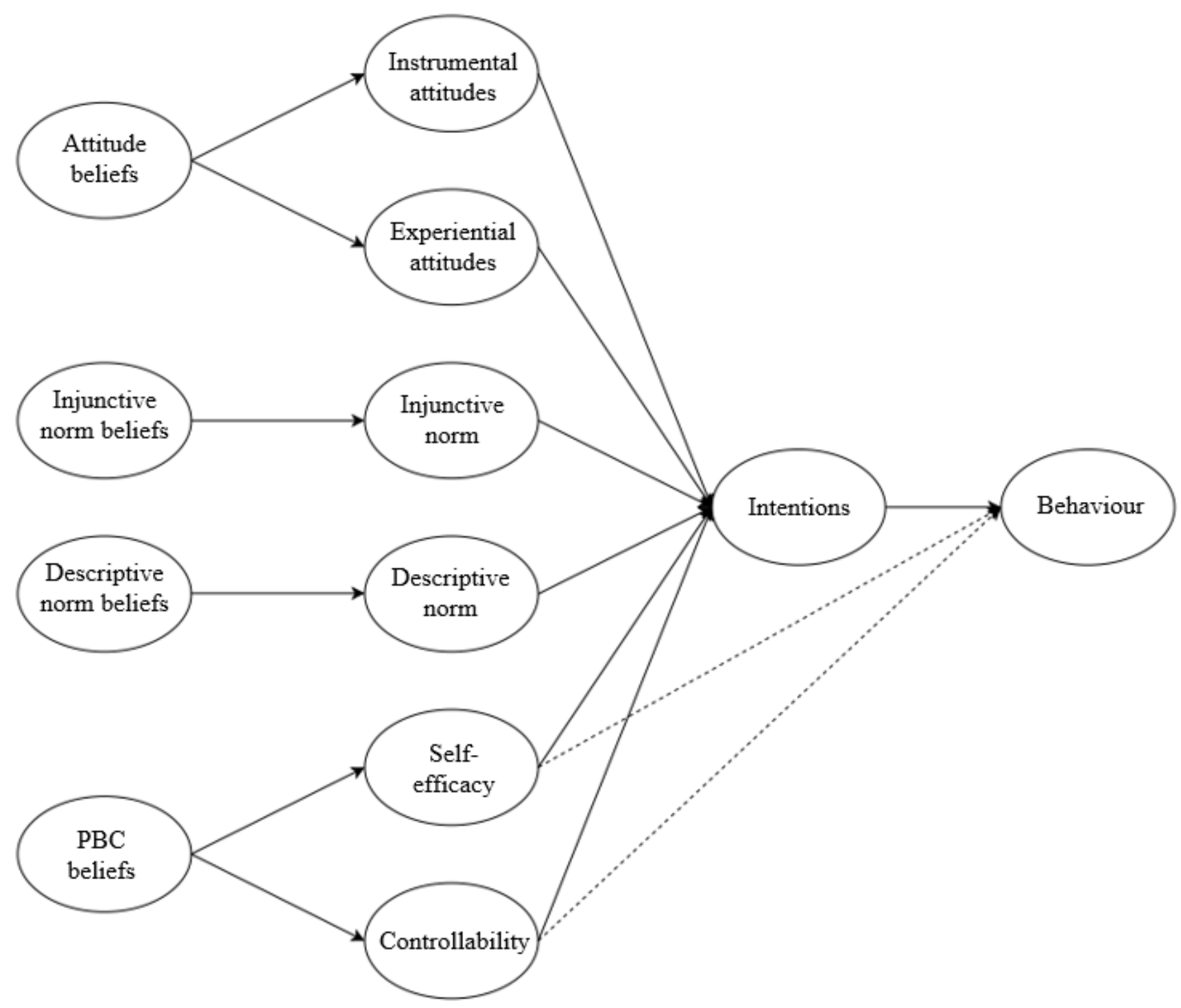

Figure 2.5. The Theory of Planned Behaviour with the cognition components divided into their theorized factors.

Further, this study included a measure of behaviour to test the assumption that intentions to prepare for natural hazards are positively and significantly associated with actual behaviour, as well as to test the theorized direct effect of perceived behavioural control (selfefficacy and controllability) on behaviour. While intentions are recognized as one of the best predictors of behaviour (Ajzen \& Madden, 1986), the association can be weak and varies depending on the type of behaviour under study (e.g., Armitage \& Conner, 2001). The existence of the intention-behaviour relationship is necessary for the success of an intervention targeting the antecedent cognitions and beliefs. Therefore, it is important to establish that this 
relationship exists for natural hazard preparation. Testing whether intentions effectively predict behaviour over time requires more resource-intensive longitudinal or experimental methods; therefore, this cross-sectional study informed the decision to fully investigate the relationship.

Finally, this study included an experimental manipulation to examine framing effects as discussed in Chapter 1. One group of participants was asked about preparing for "natural disasters" and a second group asked about preparing for "natural hazards". This allowed for a test of framing effects on the explanation of intentions as well as the association between intentions and behaviour. Despite current debate in the field around the appropriateness of the term "natural disaster" from a technical perspective, differences in how this term is perceived by the public compared to a common alternative ("natural hazard") should be identified. The results of this experimental manipulation informed the use of "natural hazard" in Study 3 and throughout the first two chapters, as well as having important implications for risk communication (discussed in Chapters 4 and 7).

As well as providing evidence for the potential of framing effects to contribute to fieldspecific discussion and research, differences found based on a specific word change support the suggestion that some of the conflicting findings within the TPB literature could be due to how studies frame the behaviours which they target. Such evidence implies that language should be used consistently between studies looking at the same behaviours and that those testing interventions should consider whether there might be a way to re-frame the target behaviour to make those interventions more successful.

Participants for Study 1 and Study 2 were recruited from the general Wellington population in line with suggestions to produce more research using non-student samples (Armitage et al., 2013). Webb and Sheeran's (2006) meta-analysis found no difference in effect sizes between student and non-student samples. However, student samples tend to demonstrate higher compliance and therefore potentially social desirability biases (Elliott \& Armitage, 2009) and would likely produce less generalizable findings given likely differences in natural hazard preparation among students compared to non-students (Levac et al., 2012).

Study 3. Study 3 built on the previous study by testing whether the developed measure of intentions predicts behaviour over time. Intentions, as well as the antecedent cognitions and beliefs, were measured at Time 1 and behaviour at Time 2, one month later. This longitudinal study provided stronger support for a causal link between intentions and behaviour regarding natural hazard preparation, although such a design is still open to third-factor problems (Webb $\&$ Sheeran, 2006). Further, Study 3 tested the two-factor distinction at the belief level. For example, experiential attitudes were explained by experiential attitude beliefs, rather than the 
general attitude belief construct used in Study 2. This distinction was also included for controllability and self-efficacy in PBC.

Additionally, while Study 2 used a single, dichotomous measure of behaviour (requesting or not requesting an earthquake planning guide), Study 3 used a range of behaviour measures. This study retained the dichotomous measure used in Study 2 for replicability purposes but included a self-report item assessing general preparation behaviour using a Likerttype response scale as well as a list of specific actions that participants could report undertaking in the time since the first survey. Data from these latter two measures were treated as continuous rather than dichotomous; intentions are argued to better predict behaviour when the two factors are the same type of variable (e.g., both dichotomous or both continuous; Topa \& Moriano, 2010). Including an objective measure, a single-item self-report measure, and a multiple-item self-report measure of behaviour also increased the reliability of inferences about the intention-behaviour association.

Study 3 also aimed to improve on common issues with non-representative samples in previous TPB research (including Studies 1 and 2 of this thesis) by using random sampling from Wellington council electoral rolls. Studies 1 and 2 employed online recruitment targeted only by geographical location, which tends to lead to younger samples with overrepresentation of women (Vinnell, 2016).

Study 4. Finally, Study 4 retrospectively evaluated the effect of an existing earthquakepreparedness intervention run both internationally and in New Zealand by the National Emergency Management Agency: the ShakeOut earthquake drill, which is described more fully in Chapter 6. Briefly, this drill encourages people to practice protective actions to use during earthquake shaking. At a designated time on a particular date, several hundred thousand people, typically in schools or workplaces, "drop, cover, and hold". The National Emergency Management Agency also encourages those in coastal areas, particularly schools, to practice tsunami evacuations as part of the drill. This study used a quantitative survey methodology adapted from similar evaluative surveys run following the drill in 2012 and 2015 (Vinnell, Wallis, Becker, \& Johnston, 2020) and used a quasi-experimental design to test whether participating in the earthquake drill led to differences in the TPB components, as well as other relevant cognitions such as fatalism bias. Further, this study tested whether participating in the drill increased knowledge of protective actions to take during shaking (the primary goal of the drill), actual use of that behaviour in earthquakes, and other related preparation actions (such as those considered in Studies 1 to 3). This evaluation provided support for the continued annual, national implementation of the drill, and identified ways in which the drill can 
improved. However, Study 4 also demonstrated that the drill has benefits for cognitions relating to preparation, including TPB components, which means that findings from both this study and Study 3 could be used to adapt the communication around the drill to increase its effectiveness.

The survey used in Study 4 is part of an evaluation programme run by the National Emergency Management Agency (NEMA), which leads the ShakeOut drill, and so includes other questions of interest to the agency which are not directly relevant to this thesis and so are not presented here. Belief measures were therefore not included in this study so that the survey was not exhaustively long. It is important to note that the findings of Studies 1, 2, and 3 did not inform the running of the 2018 ShakeOut drill; rather, the measures developed in the previous studies were used as part of a wider evaluation of the drill, run by NEMA, and including collaboration with other research institutions.

\section{Summary}

Several theories of behaviour have been used to explain preparation for natural hazards, including a number which were created for that particular context. The Theory of Planned Behaviour provides relevant factors to test in this research as well as opportunities to develop the model by addressing inconsistencies within the literature which has applied it. The following chapter reports Study 1, which included a preliminary test of the applicability of the TPB to understanding intentions to prepare for natural hazards. 


\section{Chapter 3: Study 1}

\section{Introduction}

\section{Overview}

Previous research has established that preparation for disasters can be low, even when risk perception is high, as is the case in Wellington, New Zealand (Johnston et al., 2013; Khan, Crozier, \& Kennedy, 2012). However, it is important to ensure that assumptions about a particular population and its behaviour are accurate before carrying out more in-depth research (McKenzie-Mohr, 2011). Further, the TPB is not frequently used to test intentions to prepare at a household level for natural disasters (hereafter simply "prepare" or "preparation" for the sake of brevity), with no uses in a New Zealand context. This study provides preliminary support for the capacity of the TPB to explain such intentions in the current research population and has three key aims.

First, this study aims to establish whether preparation is low in Wellington as indicated by previous research (Johnston et al., 2013), and identify which preparation actions are least taken to establish ideal behaviours to target in future interventions as is recommended (McKenzie-Mohr, 2011). Although many studies have demonstrated low levels of preparation (e.g., McClure, Spittal, Fischer, \& Charleson, 2015), it is possible that recent events such as the November 2016 Kaikōura earthquake led to an increase in preparation. Evidence suggests that any increases in preparedness tend to be short-lived, including specifically after the 2016 Kaikōura earthquake (Colmar-Brunton, 2018); however, as past behaviour is a variable of interest and this study aims to identify specific behaviours with greater potential for targeting, the data to test this assumption were available and therefore used.

Second, this study aimed to identify the key barriers to preparation among the wider population of the urban Wellington region, extending and replicating the findings of McClure et al. (2015) which recruited a modest $(N=200)$ sample in four specific locations in the region. This study used a broader, less targeted recruitment strategy and aimed to recruit a larger sample to better assess the prevalence of behavioural barriers. Finally, this study aimed to establish the relevance of the TPB variables for explaining intentions of Wellington residents to prepare for disasters at a household level to support more thorough investigations of the factors in the model as such investigations require measurement development. 


\section{Hypotheses}

The nature of this study is largely exploratory, with the aim of testing the applicability of the TPB to disaster preparation, as well as to identify current behaviour and barriers. However, enough research exists to allow for some hypotheses.

Behaviour hypotheses. Research has differentiated between survival and mitigation actions related to earthquakes (Russell, Goltz, \& Bourque, 1995), which refer to actions to help survive in the aftermath of an event and to reduce damage from the event itself, respectively. I expected to find that survival actions are more commonly done than mitigation actions, based on a number of studies demonstrating the same relationship, albeit specifically related to earthquakes (e.g., McClure et al., 2015). Support for this hypothesis will therefore confirm that the differences between the two types of actions extend beyond earthquakes to disasters more generally. Regardless of this difference, I expected overall preparation to be low, again based on a number of studies in the New Zealand context, including some research considering other natural hazards (e.g., Khan et al. 2012).

In line with the findings of McClure and colleagues (2015) and Joffe, Perez-Fuentes, Potts, and Rossetto (2016), expected that the most common reason for not engaging in a specific preparation action would be that the participant has not gotten around to completing it. This reason reflects procrastination which could be driven by a number of factors of which participants are not self-aware, such as the thoughts and beliefs about the behaviour of preparing explored in Studies 2 and 3. Allowing participants to indicate this reason for not preparing allows for a test of the assumption that people make a decision to not prepare, rather than believing that practical barriers limit their behaviour. I expected cost to only be a highranked barrier for the more expensive actions, such as purchasing disaster insurance and strengthening foundations (Terpstra \& Lindell, 2012).

Model hypotheses. Based on the findings of Ejeta, Ardalan, and Paton (2015), I expected to find a significant regression model for intentions to prepare, with significant pathways from the three components (attitudes, perceived social norms (hereafter referred to as "norms"), and perceived behavioural control) to intentions. Given that I am testing behaviour at the same time point, I also tested for direct pathways from the three components to reports of past behaviour. As past behaviour is typically a strong predictor of behaviour (Sniehotta, Scholz, \& Schwarzer, 2005), I expected that attitudes and norms would be positively associated with past behaviour. Support for the proposed direct link between PBC and behaviour (Ajzen \& Madden, 1986) is mixed, so while I did not have a specific hypothesis as to whether this path 
would be found in the current study, the finding either way provides information relevant to the theory.

As this is a preliminary test of the "two-factor" TPB constructs (Conner, Hugh-Jones, $\&$ Berg, 2011), I expected to find that the sub-components represent discrete constructs. In line with current recommendations (e.g., Ajzen, 2006a), I split the subjective norm component into descriptive and injunctive norms. I expected to find unique contributions of each norm type to the explanation of intentions. Similarly, and again consistent with the current preferred measurement of the TPB, I expected to find that the traditional PBC measure (Ajzen \& Madden, 1986) and a self-efficacy measure (Duval \& Mulilis, 1991) also represent discrete constructs in that they contribute uniquely to the explanation of intentions. As this is a preliminary study, I did not include the distinction of instrumental and experiential attitudes. This distinction is introduced in Study 2 (Chapter 4).

Demographics. This study was conducted in the Wellington Region, which has experienced a range of natural hazard events, mainly earthquakes and storm impacts, of which most participants are likely to have some knowledge and experience. Further, the majority of residents are of European descent, but other ethnic groups including Māori and those from Pacific and Asian countries comprise a considerable portion of the population. Therefore, this study also considered a range of demographic variables found inconsistently to affect disaster preparation, such as previous disaster experience (e.g., Becker, Paton, \& Johnston, 2015; Bourque et al., 2012; Lindell, 2013) and risk perception (e.g., Bourque, 2013; Johnston et al., 2013; Perry \& Lindell, 2008). For both aforementioned variables, I expected positive relationships with preparation, although the relationships are often curvilinear (e.g., Solberg, Rossetto, \& Joffe, 2010; Vinnell, 2016). Other demographics, such as age, gender, income, ethnicity, and education have in previous research correlated with disaster preparedness, although these correlations are often inconsistent between studies (see Becker et al., 2015, Lindell \& Whitney, or Solberg et al., 2010 for reviews). The testing for associations between demographic factors and disaster preparation was therefore largely exploratory. 


\section{Method}

\section{Design}

Study 1 used a correlational survey design to identify the current levels of natural disaster preparation behaviours in Wellington, as well as the factors which relate positively and negatively to those behaviours.

\section{Participants}

Study 1 used a general population sample from Wellington. Participants were recruited through social media (Facebook) so were not selected to obtain an even gender split or representative distributions on demographics such as age and ethnicity. There was no target sample size, so the survey was left open for a week (October $27^{\text {th }}, 2017$ to November $2^{\text {nd }}, 2017$ ). This allowed for the recruitment of enough participants to confidently conduct analyses but limited the data collection window to reduce potential confounds such as occurrence of natural hazard events.

Eight-hundred and twenty-six participants commenced the survey. Three did not consent to participate and 35 did not live in Wellington so were directed to the end of the survey without answering further questions. A further three participants were excluded for being under the required age of 18. Finally, 63 participants who did not complete at least two scales were also excluded from any analyses. This left useable data from 722 participants.

Participants' ages ranged from 18 to 63 years old, with a mean of 30.44 years $(S D=$ 10.60), and 198 participants not providing their age. Of the 545 participants who indicated their gender, 64 were men (11.7\%), 478 were women (87.7\%), and 3 were non-binary (e.g., agender; 0.6\%). Three hundred and seventy-six (52.1\%) identified as New Zealand European/Pākehā, $118(16.3 \%)$ as Māori, 63 (8.7\%) as Pasifika, 37 (5.1\%) as European, 35 (4.8\%) as Asian, 11 $(1.5 \%)$ as North American, $5(0.7 \%)$ as South/Latin American, $3(0.4 \%)$ as African, and 26 $(3.6 \%)$ as other ${ }^{3}$. Of the 544 participants who indicated their highest level of education, 55 (7.6\%) had not completed secondary school, 171 (31.4\%) had a secondary school qualification, $107(19.7 \%)$ had a polytechnic or trade certificate, $135(24.8 \%)$ had an undergraduate degree, and $76(13.9 \%)$ had a postgraduate degree.

The modal level of annual income before tax was below \$20,000 (29.8\%), with $13.3 \%$ earning between $\$ 20,000$ and $\$ 29,999,10.2 \%$ between $\$ 30,000$ and $\$ 39,999,10.5 \%$ between $\$ 40,000$ and $\$ 49,999,9.6 \%$ between $\$ 50,000$ and $\$ 59,999,5.2 \%$ between $\$ 60,000$ and $\$ 69,999$, and $8.8 \%$ earning $\$ 70,000$ or more. One hundred and eighty-one participants did not

\footnotetext{
${ }^{3}$ Participants were able to indicate identification with multiple ethnicities
} 
respond to the question, and a further 68 responded that they preferred not to say their income. Approximately one-third (33.2\%) of respondents indicated that their household costs exceeded their incomes, $30.2 \%$ indicated that their income roughly matched their household costs, and $36.7 \%$ indicated that their income exceeded their household costs.

Most respondents were renting (57.6\%), with the next most common housing situations being living in a home that they own (16.7\%) and living in their parents' home (12.3\%). $6.2 \%$ lived in social housing and $4.2 \%$ in a hostel or halls of residence, with the final $2.9 \%$ responding "Other". Most participants who indicated housing type lived in a free-standing house $(64.3 \%)$, a unit or flat $(22.5 \%)$, or an apartment $(11.5 \%)$.

\section{Materials}

An online survey included a range of measures adapted from previous research, assessing the following variables relating to natural disasters: risk perception, past experience, preparation actions already taken (survival and mitigation), intentions to take further preparation actions, barriers to preparing, personal approval of preparing, perceived descriptive norms, perceived injunctive norms, attitudes towards preparing, PBC, self-efficacy, and response-efficacy. I also obtained demographic information, including: age, gender, ethnicity, education, income, location (suburb), living situation, and housing situation. The information sheet and the full survey are presented in Appendices 3.A and 3.B, respectively.

Before the first measure, participants were given the following information:

"This survey will ask a number of questions about natural disasters. For the purpose of this research, a natural disaster is an event or force of nature, such as an earthquake, flood, tsunami, landslide, wildfire, storm, and volcanic eruption, which has severely negative consequences such as causing a lot of damage, injury, and/or disruption to lifelines (e.g., roads, water, electricity, food and medical supplies)."

Risk perception. This six-item measure was adapted from Terpstra and Lindell (2012). All items used a 5-point Likert-type response scale, ranging from "Very unlikely" to "Neither likely nor unlikely" to "Very likely". The first item asks, "Where you live, how likely is it that a natural disaster will occur in the next five years?" to assess perception of the likelihood of the risk occurring. Participants are then given the instruction: "Suppose that a natural disaster has occurred in your area. How likely do you think it is that the following will occur?". This instruction is followed by five types of negative outcome, to assess the perception of the likelihood of risk consequences occurring. These are: "Major damage to public facilities (e.g., roads and parks) in your area"; "Supplies (electricity, communications, water, etc.) will be interrupted"; "Major damage to your home/possessions"; "You and/or your family face a life- 
threatening situation"; and "A prolonged disruption of your daily life (work and other daily activities)". The mean of the five consequence items (between 1 and 5) is multiplied by the first risk item (between 1 and 5) to give a risk perception score between 1 (lowest) and 25 (highest). The five consequence items demonstrated acceptable internal reliability, Cronbach's $\alpha=.86$.

Disaster experience. Of this three-item measure, two questions were adapted from McClure, Willis, Johnston, and Recker (2011), and the third added for this study. Adaptations included bolding the key words in the question. The questions asked: "Have you or someone close to you suffered damage to your home or possessions in a past natural disaster?"; "Have you or someone close to you been harmed in a past natural disaster?"; and "Have you experienced natural events which didn't cause you harm or damage but made you feel scared or vulnerable?". All items used a 7-point Likert-type response scale, ranging from "Never" to "A moderate amount/Moderately/A moderate number" (respectively) to "A lot/Severely/A lot" (respectively). The scores for each item are averaged to create a scale mean between 1 (lowest) and 7 (highest). This scale demonstrated reasonable internal reliability for a three-item measure, $\alpha=.57$. Deleting any of the items would not increase the alpha, and the corrected item-total correlations were within an acceptable range (.31 to .47).

Disaster preparation. This measure presented a list of 18 behaviours to increase preparedness for a range of natural disasters, drawn from several scientific sources (McClure et al., 2015; Mulilis, Duval, \& Lippa, 1990; Spittal, Walkey, McClure, Siegert, \& Ballantyne, 2006) and various government sources (such as getready.govt.nz and National Emergency Management Agency leaflet material). Participants were presented with the following instructions:

"Please indicate which of these preparations you or someone in your household have made for the specific purpose of a natural disaster. If you've done one of these in the past but can no longer benefit from it (e.g., stored food years ago which you've since thrown out) please respond "No". If you have done part of the action (e.g., purchased a torch but not a batterypowered radio) please respond "Partly"."

For each behaviour, participants could respond "Yes", "No", "Partly", "Unsure", or "Not applicable". The instructions and the behaviours were worded so as to make the behaviours applicable for as many people as possible: for example, including the behaviour of others in the household or including asking the landlord to carry out actions which tenants cannot. The 18 behaviours are: 
- Store water

- Store non-perishable food

- Make an emergency kit

- Make an emergency plan (e.g., knowing where to meet family)

- Store supplies (such as plastic bags and toilet paper) to use as an emergency toilet

- Purchase items to use if power is lost such as a torch, radio, or gas cooker

- Cloud store important documents and/or photos on an internet server

- Purchase natural disaster insurance

- Seek out information about the different natural hazard risks posed to my home

- Have the strength of my building checked (or ask landlord to do the same)

- Fasten tall furniture to the wall

- Purchase a water tank

- Secure movable objects in my home (such as computers and TVs)

- Strengthen my house/its foundations (or ask landlord to do the same)

- Ensure that heavy objects are stored on the floor and at the bottom of cupboards

- Identify people in my neighbourhood who need checking up on in the event of a natural disaster

- Store enough emergency supplies to help others not in my household

- Volunteer my time to help my community in the event of a disaster e.g., Community Emergency Hubs

At the bottom of the page, participants were given the option to list any other behaviours they had undertaken to prepare for natural disasters that were not presented in the question. Only 93 participants responded to this question; of those, 45 said they had not done other actions and 23 reported another action which had already been included in the above list. The actions reported by the remaining 25 participants are listed below but are not analysed given the small sample size.

Preparation intentions and barriers. Participants were presented with a list of the behaviours that they indicated they had not carried out. To measure intentions to carry out those behaviours, participants were presented with the instruction "For the preparation actions you have NOT taken, please indicate if you intend to do them" and the options "Definitely not", "Probably not", "Might or might not", "Probably yes", and "Definitely yes". To identify barriers to preparation, participants were presented with the instruction: "For the preparation 
actions you have NOT taken, which of the following reasons best explains why?" and the options "It's too expensive", "It's not going to help in a disaster", "I have more important things to do", "I don't need to do it", "I hadn't thought about doing it", "I haven't got around to it", "I can't be bothered", and "Other" (McClure et al., 2015).

Personal norm. To measure the participant's own approval of preparation, four items repeated the same question with increasingly broad reference groups. The question read "I think my family and friends / people in my neighbourhood / Wellingtonians / New Zealanders should prepare for a natural disaster". 7-point Likert response scales were used, ranging from "Strongly disagree" to "Neither agree nor disagree" to "Strongly agree". The average of the items was used to create a mean personal norm score between 1 (lowest) and 7 (highest), with the higher number indicating a stronger approval of others' preparation behaviour. This scale demonstrated good internal reliability, Cronbach's $\alpha=.938$, and the corrected item-total correlations ranged from .793 to .882, indicating that the items are measuring similar perceptions.

Descriptive norm. Similar to the personal norm measure, participants were asked to make the same judgment for four reference groups ("Friends and family", "People in my neighbourhood", "People in Wellington", and "People in New Zealand"). For each group, participants used a slider bar ranging from 0 to 100 to indicate what percentage of the members of each group they think have prepared for a natural disaster. Precisely, the instructions presented were "Please use the slider bars to indicate roughly what percentage of people you think have "prepared" for a natural disaster (done many of the actions given earlier in the survey) for each group." The averages of all items were used to create a scale mean between 0 (lowest) and 100 (highest) where a higher score indicates a stronger descriptive norm. Research examining descriptive norms have begun to use percentage estimates as responses to obtain better data than a Likert-type response scale (e.g., Mortensen et al., 2017; Sparkman \& Walton, 2017). Providing a different type of response also intended to reduce fatigue and limit boredom as pilot testing indicated that the norm measures became repetitive. This scale demonstrated acceptable internal reliability for a four-item measure, Cronbach's $\alpha=.781$, which would not be increased by deleting any of the items. The corrected item-total correlations were within an acceptable range (.517 to .691).

Injunctive norm. As with the previous norm measures, this scale used four items each asking the same question but with a different reference group. The question read "How strongly do you think your family and friends / people in your neighbourhood / Wellingtonians / New Zealanders approve of other people preparing for a natural disaster?" These items used 
a 7-point Likert response scale, ranging from "Strongly disapprove" to "Neither approve nor disapprove" to "Strongly approve". The averages of all items were used to create a scale mean between 1 (lowest) and 7 (highest) where a higher score indicates a stronger injunctive norm. This scale demonstrated good internal reliability, Cronbach's $\alpha=.870$, which would not be increased by deleting any items. The corrected item-total correlations were higher for this scale than the descriptive norm scale (range: .656 to 773.) but are still acceptable.

Attitudes. This measure used a semantic differential scale adapted from Ajzen and Madden (1986). Eight pairs of adjectives were presented, with one of the pair at each end of a 7-point response scale. The pairs were derived through a small-sample survey $(N=15)$ via Facebook asking what adjectives participants would use to describe disaster preparation, selecting the eight most common responses, and identifying the antonym for each word. Participants in Study 1 were given the stem "Preparing for natural disasters is:" and then the list of adjectives: "Useless", "Useful”; "Unimportant”, "Important”; "Unnecessary”, "Necessary"; "Easy", "Difficult"; "Pointless", "Valuable"; "Smart", "Stupid"; "Simple", "Complicated"; and "Unhelpful”, "Helpful”. Items 4, 6, and 7 were reverse-coded so that for all items a score of 1 indicated a negative attitude and a score of 7 indicated a positive attitude. The average of all items was calculated for each participant to create a scale mean between 1 (lowest) and 7 (highest), where a higher score indicates a more positive attitude towards disaster preparation. This scale demonstrated acceptable internal reliability, Cronbach's $\alpha=$ .803 , although this reliability could be increased by deleting two of the items: Easy/Difficult and Simple/Complicated. As this scale represents untested adjective pairs, a principal components analysis was run to provide further support for removing the two above items in order to refine the measure. Consistent with the reliability analysis, the PCA revealed two components (Eigenvalues: $4.21 ; 1.49$ ), with the second component comprising the two items stated above. After removing these items from the scale, Cronbach's $\alpha$ increased to .911, which could not be improved by removing any further items. All subsequent results used the refined, six-item measure in analysis.

Perceived behavioural control. This three-item measure was also adapted from Ajzen and Madden (1986), with minor wording changes made. The first item asked, "How much control do you have over whether you prepare for a major disaster?" and used a 7-point Likerttype response scale ranging from "Very little control" to "A moderate amount" to "Complete control". The second item read "For me to prepare for a natural disaster is..." and also had a 7-point response scale but with the anchors "Easy", "Neither easy nor difficult", and "Difficult". The final item read "If I wanted to I could prepare for a natural disaster" and had 
"Definitely could", "Neither could nor couldn't", and "Definitely couldn't" as the anchors. This last item was reverse coded before analyses. The average of all items for each participant was used to create a scale mean between 1 (lowest) and 7 (highest), with higher scores indicating more perceived control over the behaviour. This scale demonstrated acceptable internal reliability for a three-item measure, Cronbach's $\alpha=.609$, and acceptable corrected item-total correlations (range: 339 to .494 ).

Self- and response-efficacy. This measure was similar to that used to measure preparation behaviours and adapted from Mulilis et al. (1990). Participants were given the following instructions:

"In this question, we would like to ask two related questions. First, for the actions you HAVE done, please indicate how difficult they were, and for the actions you have NOT done, how difficult you think they would be. Please then indicate how effective you think each action is at helping you survive a natural disaster and/or reducing damage in a natural disaster."

Participants were then presented with the same list of 18 preparation behaviours. For each behaviour they rated the difficulty, from "Easy" to "Neutral" to "Difficult", and the effectiveness, from "Not at all" to "Neutral" to "Very". The difficulty responses for each participant were reverse-coded then averaged to create a scale mean between 1 (lowest) and 5 (highest), with higher scores indicating stronger beliefs of self-efficacy. The responses for effectiveness were also averaged, so that each participant had a mean score between 1 and 5 , with higher scores indicating stronger beliefs of response-efficacy.

Demographics. Participants responded to demographic questions about their age, gender, ethnicity, education, income, disposable income, suburb, living situation, and housing situation. The full items are presented in Appendix 3.B. These questions were based on those used in the national Census.

\section{Procedure}

The study was delivered online using the survey platform Qualtrics between October $24^{\text {th }}$ and November $6^{\text {th }}, 2017$. Participants could undertake the survey on any Internet-enabled device. Information about the study and the contact details of the researchers were provided before commencement (see Appendix 3.A). Participants were also required to indicate their consent and that they were 18 years or older before proceeding to the survey. If they responded that they did not consent or were below the required age, they were automatically taken to the final completion page of the survey. Further, before reaching the first scale, participants were asked "Do you currently live in the Wellington region (including Wellington City, Upper and 
Lower Hutt, and Porirua)?" Any participants who responded "No" to this question were also automatically taken to the end of the survey.

Participants who completed the survey were provided with a debriefing page after the final questions. In order to limit the time investment required by participants, all of them completed the risk perception scale, the disaster experience scale, the three norms measures, and the demographic questions. A randomly selected half of the participants also completed the TPB measures (attitudes, perceived behavioural control, and self- and response-efficacy). The other half completed a different set of measures not reported here. Completion took approximately 15 minutes, and participants were thanked with the opportunity to enter a prize draw for one of three $\$ 100$ supermarket gift cards by following a link to a second Qualtrics survey, meaning that their identifying data could not be linked to their questionnaire responses. This study was granted approval by the School of Psychology Human Ethics Committee under the delegated authority of the Victoria University of Wellington Human Ethics Committee (approved: $17^{\text {th }}$ October 2017; reference number: 0000025441). All analyses were completed using SPSS Version 25.

\section{Results}

\section{Descriptive Statistics}

Table 3.1 presents the statistics for the scale items, as well as levels of preparation. Overall, participants, as expected, perceived a moderate to high risk of disasters, with the means $(15.51, S D=5.40)$ significantly above the midpoint, $t(719)=12.49, p<.01, d=.93$. Experience of disasters was also low $(M=2.86, S D=1.22$; difference from the midpoint, $t(25.15), p<.01, d=1.88)$. 
Table 3.1

Means and standard deviations of scale variables.

\begin{tabular}{lccccccc}
\hline Variable & $N$ & Mean & SD & Range & Midpoint & \multicolumn{2}{l}{ df } \\
\hline Risk Perception & 720 & 15.51 & 5.40 & $1-25$ & 13 & $12.49^{* *}$ & 719 \\
Disaster Experience & 721 & 2.86 & 1.22 & $1-7$ & 4 & $25.15^{* *}$ & 720 \\
Preparation & & & & & & & \\
Survival Preparation & 681 & 4.99 & 3.8 & $0-18$ & 9 & $27.55^{* *}$ & 680 \\
Mitigation Preparation & 681 & 3.01 & 2.30 & $0-8$ & 4 & $11.26^{* *}$ & 680 \\
& 679 & 1.50 & 1.64 & $0-7$ & 3.5 & $31.89^{* *}$ & 678 \\
Intentions & & & & & & & \\
Survival Intentions & 638 & 2.93 & .76 & $1-5$ & 2.5 & $14.32^{* *}$ & 637 \\
Mitigation Intentions & 602 & 3.25 & .91 & $1-5$ & 2.5 & $17.68^{* *}$ & 601 \\
& 605 & 2.73 & .89 & $1-5$ & 2.5 & $6.31^{* *}$ & 604 \\
Personal Norm & 618 & 6.29 & .99 & $1-7$ & 4 & $57.75^{* *}$ & 617 \\
Descriptive norm & 611 & 44.99 & 17.33 & $0-100$ & 50 & $-7.15^{* *}$ & 610 \\
Injunctive norm & 610 & 5.99 & .88 & $1-7$ & 4 & $56.12^{* *}$ & 609 \\
Attitude & 245 & 5.99 & .88 & $1-7$ & 4 & $27.76^{* *}$ & 244 \\
PBC & 277 & 4.78 & 1.20 & $1-7$ & 4 & $10.87^{* *}$ & 276 \\
Self-Efficacy & 266 & 3.32 & .77 & $1-5$ & 2.5 & $17.25^{* *}$ & 201 \\
Response-Efficacy & 202 & 3.97 & .73 & $1-5$ & 2.5 & $28.81^{* *}$ & 265 \\
\hline$\quad$ Note. & & & & & & &
\end{tabular}

Note. $*$ significant at the level of $\mathrm{p}<.05 . * *=$ significant at the level of $\mathrm{p}<.01$.

Preparation. As predicted, participants were relatively unprepared for a disaster, with the mean number of preparation actions out of the 18 presented items being 4.99 ( $S D=3.80$; difference from the midpoint $t(680)=27.55, p<.01, d=2.11)$. As expected, participants had completed more survival actions, $37.63 \%$ of the actions on average, compared to $21.4 \%$ for mitigation actions ${ }^{4}$. For both types of actions, participants had still completed significantly fewer than half of the presented list (survival: difference from midpoint, $t(680)=11.26, p<.01$, $d=.86$; mitigation: difference from midpoint, $t(678)=31.89, p<.01, d=2.45$ ).

\footnotetext{
${ }^{4}$ Of the 18 total items, eight were survival actions while seven were mitigation actions so percentages allow for a direct comparison of prevalence, as part of the difference in the means reflects the differences in possible totals.
} 
Table 3.2 presents the frequency of each individual preparation behaviour (see also Table 3.3 for behaviours in rank order). The most commonly completed action was storing water, but this was still only done by about half (54.8\%) of participants. Similarly, about half of the participants reported having a store of food (50.0\%). Of the 25 participants who reported undertaking another action not included in the list, most common were keeping petrol either stored or having at least half a tank in their car, making provisions for pets, having supplies somewhere other than their house including sheds and at work, and storing camping equipment. 
Table 3.2

Frequency of preparation behaviours and mean scores of intentions, judgments of difficulty, and judgments of effectiveness.

\begin{tabular}{|c|c|c|c|c|c|c|c|c|c|c|c|}
\hline \multirow[b]{2}{*}{ Action } & \multicolumn{5}{|c|}{ Preparation } & \multicolumn{2}{|l|}{ Intention } & \multirow{2}{*}{$\begin{array}{c}\text { Difficulty } \\
\text { Mean }\end{array}$} & \multicolumn{3}{|c|}{ Effectiveness } \\
\hline & Yes & No & Partly & Unsure & N/A & Mean & $S D$ & & $S D$ & Mean & $S D$ \\
\hline Stored Water & 54.8 & 29.2 & 14.0 & 1.5 & 0.6 & 3.38 & 1.11 & 4.00 & 1.20 & 4.59 & .81 \\
\hline Stored Food & 50.0 & 29.0 & 20.3 & 0.6 & 0.1 & 3.39 & 1.11 & 4.07 & 1.06 & 4.59 & .78 \\
\hline Emergency kit & 43.4 & 33.1 & 22.2 & 1.0 & 0.1 & 3.65 & 1.07 & 3.55 & 1.20 & 4.46 & .88 \\
\hline Emergency plan & 35.2 & 39.7 & 22.7 & 1.6 & 0.7 & 3.58 & 1.07 & 3.97 & 1.09 & 4.27 & .96 \\
\hline Emergency toilet & 33.8 & 46.7 & 17.7 & 1.5 & 0.3 & 3.38 & 1.12 & 4.06 & 1.07 & 4.18 & 1.01 \\
\hline Battery torch etc. & 43.9 & 31.8 & 22.6 & 1.3 & 0.4 & 3.37 & 1.11 & 3.66 & 1.20 & 4.35 & .92 \\
\hline Water tank & 9.7 & 84.8 & 2.7 & 1.0 & 1.8 & 2.43 & 1.13 & 2.22 & 1.22 & 3.80 & 1.18 \\
\hline Cloud store & 32.0 & 50.4 & 14.6 & 2.4 & 0.6 & 3.24 & 1.07 & 3.71 & 1.25 & 3.85 & 1.11 \\
\hline Disaster insurance & 13.4 & 70.6 & 4.0 & 10.6 & 1.3 & 2.27 & .97 & 2.45 & 1.26 & 3.48 & 1.29 \\
\hline Risk information & 22.4 & 59.1 & 14.7 & 3.1 & 0.7 & 2.89 & 1.08 & 3.16 & 1.22 & 3.57 & 1.12 \\
\hline Building check & 27.2 & $\mathbf{5 7 . 0}$ & 7.1 & 7.2 & 1.5 & 2.58 & 1.09 & 2.62 & 1.35 & 3.69 & 1.10 \\
\hline Fasten furniture & 19.3 & 62.2 & 11.5 & 1.9 & 5.0 & 2.93 & 1.11 & 3.10 & 1.21 & 4.09 & .99 \\
\hline Secure objects & 20.1 & 62.2 & 14.8 & 1.5 & 1.5 & 2.92 & 1.09 & 3.31 & 1.19 & 3.94 & 1.01 \\
\hline Strengthen & 11.1 & 69.1 & 5.8 & 7.5 & 6.5 & 2.40 & 1.11 & 2.62 & 1.36 & 3.90 & 1.04 \\
\hline Heavy objects low & 36.9 & 36.9 & 22.9 & 2.1 & 1.2 & 3.07 & 1.13 & 3.10 & 1.08 & 3.92 & 1.03 \\
\hline Identify neighbours & 22.9 & 62.5 & 11.7 & 1.9 & 1.0 & 2.84 & 1.06 & 3.31 & 1.29 & 3.76 & 1.13 \\
\hline Extra supplies & 14.1 & 70.8 & 12.2 & 2.2 & 0.6 & 2.86 & 1.07 & 3.01 & 1.34 & 3.88 & 1.14 \\
\hline Volunteer & 12.8 & 72.3 & 8.2 & 3.7 & 3.0 & 2.66 & .95 & 3.09 & 1.31 & 3.68 & 1.20 \\
\hline
\end{tabular}

Note. All numbers under the "Preparation" column are valid percentages based on the participants who responded to each item. Numbers in bold are the most common response for each behaviour. 
Table 3.3

Rank order of preparation behaviours.

\begin{tabular}{|c|c|c|}
\hline \multirow[b]{2}{*}{ Action } & \multicolumn{2}{|c|}{ Preparation } \\
\hline & Yes & Partly \\
\hline Stored Water & 1 & 10 \\
\hline Stored Food & 2 & 5 \\
\hline Emergency kit & 4 & 4 \\
\hline Emergency plan & 6 & 2 \\
\hline Emergency toilet & 7 & 6 \\
\hline Battery torch etc. & 3 & 3 \\
\hline Water tank & 18 & 18 \\
\hline Cloud store & 8 & 9 \\
\hline Disaster insurance & 15 & 17 \\
\hline Risk information & 11 & 8 \\
\hline Building check & 9 & 15 \\
\hline Fasten furniture & 13 & 13 \\
\hline Secure objects & 12 & 7 \\
\hline Strengthen & 17 & 16 \\
\hline Heavy objects low & 5 & 1 \\
\hline Identify neighbours & 10 & 12 \\
\hline Extra supplies & 14 & 11 \\
\hline Volunteer & 16 & 14 \\
\hline
\end{tabular}

Note. Rankings are based on valid percentages of participants who responded to each item.

Intentions. Table 3.2 presents mean intentions to carry out each preparation action. Participants on average had moderate intentions to carry out the preparation behaviours they had not yet done $(M=2.93, S D=.76$; difference from the midpoint of $3, t(637)=14.32, p<$ $.01, d=1.14)$. When splitting these behaviours into survival and mitigation actions, participants had moderate intentions for both (survival: $M=3.25, S D=.91$; difference from the midpoint, $t(601)=17.68, p<.01, d=1.44$; mitigation: $M=2.73, S D=.89$; difference from the midpoint, $t(604)=6.31, p<.01, d=.51)$. However, participants had significantly stronger intentions to carry out survival actions than mitigation actions, $t(575)=11.86, p<.01, d=.99$. 
TPB components. Participants had a strongly positive attitude towards disaster preparation with the mean $(M=5.99, S D=.88)$ significantly above the midpoint, $t(244)=$ 27.76, $p<.01, d=3.55$. Similarly, they also strongly approved of other people preparing for disasters, with the mean for personal norms $(M=6.29, S D=.99)$ significantly above the midpoint, $t(617)=57.75, p<.01, d=4.65$. These strong personal norms were reflected in strong injunctive norms $(M=5.99, S D=.88$; difference from the midpoint, $t(609)=56.12, p$ $<.01, d=4.55)$. Participants estimated less than half of Wellingtonians having done "many" of the 18 items presented $(M=44.99), S D=17.33$; difference from midpoint, $t(610)=7.15, p$ $<.01, d=.58$ ). Although this finding represents a minority descriptive norm (i.e., less than half of the referent group having engaged in the behaviour), this perception appears to be biased in the direction of the behaviour seeming more common than it actually is. Participants believed that they had a moderate amount of control over preparing for disasters, with the mean $(M=$ 4.78, $S D=1.20)$ significantly above the midpoint, $t(276)=10.87, p<.01, d=1.31)$. 


\section{Barriers.}

Table 3.4

Frequency of indicated barriers to preparation behaviour.

\begin{tabular}{|c|c|c|c|c|c|c|c|c|}
\hline Action & Cost & Won't help & Less important & No need & Hadn't thought & Haven't got around & Can't be bothered & Other \\
\hline Stored Water & 10.9 & 1.6 & 3.3 & 2.2 & 26.1 & 40.8 & 7.1 & 8.2 \\
\hline Stored Food & 20.8 & 1.1 & 3.9 & 0.6 & 25.3 & 36.0 & 5.6 & 6.7 \\
\hline Emergency kit & 25.2 & 0.5 & 2.4 & 1.9 & 22.8 & 38.8 & 6.3 & 1.9 \\
\hline Emergency plan & 2.1 & 4.2 & 2.9 & 5.4 & 40.2 & 37.7 & 1.7 & 5.9 \\
\hline Emergency toilet & 8.7 & 2.8 & 4.9 & 1.7 & 47.2 & 28.5 & 3.1 & 3.1 \\
\hline Battery torch etc. & 29.9 & 1.0 & 3.0 & 0.5 & 30.5 & 29.9 & 3.6 & 1.5 \\
\hline Water tank & 50.3 & 1.9 & 2.6 & 7.3 & 16.6 & 12.6 & 2.4 & 6.2 \\
\hline Cloud store & 2.3 & 4.9 & 4.9 & 5.5 & 46.9 & 25.2 & 4.9 & 5.5 \\
\hline Disaster insurance & 51.4 & 3.2 & 1.4 & 7.8 & 24.4 & 5.8 & 3.2 & 2.8 \\
\hline Risk information & 6.7 & 4.8 & 5.6 & 6.4 & 45.4 & 17.4 & 8.4 & 5.3 \\
\hline Building check & 17.0 & 3.7 & 2.6 & 9.9 & 39.2 & 10.8 & 4.5 & 12.2 \\
\hline Fasten furniture & 5.2 & 1.6 & 4.4 & 9.4 & 22.1 & 33.0 & 10.6 & 13.8 \\
\hline Secure objects & 4.1 & 3.6 & 2.6 & 7.8 & 24.4 & 37.6 & 12.4 & 7.5 \\
\hline Strengthen & 25.0 & 1.9 & 1.4 & 12.7 & 31.4 & 9.7 & 4.5 & 13.4 \\
\hline Heavy objects low & 2.7 & 2.2 & 2.7 & 6.2 & 44.2 & 25.7 & 12.4 & 4.0 \\
\hline Check neighbours & 0.3 & 0.8 & 4.2 & 6.0 & 55.6 & 18.6 & 7.1 & 7.3 \\
\hline Extra supplies & 25.4 & 0.7 & 3.0 & 4.3 & 42.6 & 14.6 & 4.8 & 4.6 \\
\hline Volunteer & 0.9 & 1.1 & 13.9 & 3.6 & 43.0 & 15.9 & 10.8 & 10.8 \\
\hline
\end{tabular}

Note. All numbers are valid percentages based on the participants who responded to each item. Numbers in bold are the most common response for each behaviour. 
Table 3.5

Rank order of preparation behaviour barriers.

\begin{tabular}{|c|c|c|c|c|c|c|c|c|}
\hline Action & Cost & Won't help & Less important & No need & Hadn't thought & Haven't got around & Can't be bothered & Other \\
\hline Stored Water & 3 & 8 & 6 & 7 & 2 & 1 & 5 & 4 \\
\hline Stored Food & 3 & 7 & 6 & 8 & 2 & 1 & 5 & 4 \\
\hline Emergency kit & 2 & 8 & 5 & $6=$ & 3 & 1 & 4 & $6=$ \\
\hline Emergency plan & 7 & 5 & 6 & 4 & 1 & 2 & 8 & 3 \\
\hline Emergency toilet & 3 & 7 & 4 & 8 & 1 & 2 & $5=$ & $5=$ \\
\hline Battery torch etc. & $2=$ & 7 & 5 & 8 & 1 & $2=$ & 4 & 6 \\
\hline Water tank & 1 & 8 & 6 & 4 & 2 & 3 & 7 & 5 \\
\hline Cloud store & 8 & $5=$ & $5=$ & $3=$ & 1 & 2 & $5=$ & $3=$ \\
\hline Disaster insurance & 1 & $5=$ & 8 & 3 & 2 & 4 & $5=$ & 7 \\
\hline Risk information & 4 & 8 & 6 & 5 & 1 & 2 & 3 & 7 \\
\hline Building check & 2 & 7 & 8 & 5 & 1 & 4 & 6 & 3 \\
\hline Fasten furniture & 6 & 8 & 7 & 5 & 2 & 1 & 4 & 3 \\
\hline Secure objects & 6 & 7 & 8 & 4 & 2 & 1 & 3 & 5 \\
\hline Strengthen & 2 & 7 & 8 & 4 & 1 & 5 & 6 & 3 \\
\hline Heavy objects low & $6=$ & 8 & $6=$ & 4 & 1 & 2 & 3 & 5 \\
\hline Check neighbours & 8 & 7 & 6 & 5 & 1 & 2 & 4 & 3 \\
\hline Extra supplies & 2 & 8 & 7 & 6 & 1 & 3 & 4 & 5 \\
\hline Volunteer & 8 & 7 & 3 & 6 & 1 & 2 & $4=$ & $4=$ \\
\hline
\end{tabular}

Note. Rankings are based on valid percentages of participants who responded to each item. 
Table 3.4 presents the valid percentages of participants who selected each option as the best-fitting reason for not carrying out the preparation behaviour. Table 3.5 presents the rank order of the options based on the percentage information. Across behaviours, the most frequent reason given was not thinking about it (11 actions), followed by not getting around to it (five items). As expected, cost was only the most common barrier for two items, both of which would logically be perceived as expensive: purchasing a water tank and purchasing disaster insurance. For most actions, few participants indicated the barrier of thinking it "won't help", that they have more important things to do (with the exception of volunteering), and not needing to do it (with the exception of strengthening). This suggests that people think that preparing is necessary, important, and helpful, consistent with the strongly positive attitudes and personal norms reported above.

Not being bothered to do it was also not commonly a barrier, except for four actions where over $10 \%$ of participants reported it as their reason for not carrying out that action. These actions (i.e., fastening furniture, securing movable objects, putting heavy objects low down, and volunteering) are all relatively inexpensive (or free) to carry out, and require little effort to do (the volunteering behaviour referred to "in the event of a disaster", so participants potentially considered the act of registering as simple while putting less consideration towards the effort required should they be needed). Overall, these barriers show that most people are not acting because they either had not thought about carrying out a particular action, or that they had not gotten around to it, reflecting the findings of McClure et al. (2015). 


\section{Inferential Statistics}

\section{Correlations.}

Table 3.6

Correlations between factors related to natural disaster preparation.

\begin{tabular}{|c|c|c|c|c|c|c|c|}
\hline & Risk perception & Experience & Personal norm & PDN & PIN & Attitude & $\mathrm{PBC}$ \\
\hline Risk perception & - & $.185^{* *}$ & $.240 * *$ & .069 & $.110 * *$ & .091 & .037 \\
\hline Experience & & - & $.165 * *$ & .068 & .011 & .080 & -.026 \\
\hline Personal norm & & & - & $.122 * *$ & $.312 * *$ & $.244 * *$ & .102 \\
\hline Descriptive norm & & & & - & $.195 * *$ & .093 & .116 \\
\hline Injunctive norm & & & & & - & $.292 * *$ & $.233 * *$ \\
\hline Attitude & & & & & & - & $.270 * *$ \\
\hline PBC & & & & & & & - \\
\hline
\end{tabular}

Note. $\mathrm{PBC}=$ Perceived behavioural control.

$*=$ significant at the level of $\mathrm{p}<.05 . * *=$ significant at the level of $\mathrm{p}<.01$. 
Table 3.6 presents the correlations between factors relating to natural disaster preparation. Risk perception (Johnston et al., 2013) and disaster experience (Becker et al., 2014) have been included as previous research shows they can predict preparation behaviour, although there is mixed evidence as to whether they are causal factors (Bourque, 2013; Bourque et al., 2012; Lindell, 2013; Perry \& Lindell, 2008; Solberg et al., 2010). Risk perception and experience were significantly positively correlated, although the relationship was weak, $r=$ $.185, p<.01$. Both variables were also positively and significantly correlated with personal norms (perception: $r=.240, p<.01$; experience: $r=.165, p<.01$ ). Only risk perception correlated with injunctive norms, although again this relationship was weak, $r=.110, p<.01$. This supports the literature suggesting the experience of a disaster can increase risk perceptions (Becker et al., 2014), and demonstrates that largely these variables are not particularly relevant to the TPB model.

Personal norms positively correlated with both descriptive norms, $r=.122, p<.01$, and injunctive norms, $r=.312, p<.01$, although the former relationship was weaker. This suggests that people who approve more of others preparing are more likely to think that those others also approve. This finding could also represent clustering, whereby people tend to associate more with those who are similar to them. Therefore, people who approve of disaster preparation might be more likely to interact with others who think the same. This possibility is undermined by the inclusion of four different reference groups in the norms measure. As expected, personal norms also positively correlated with attitude, $r=.244, p<.01$. Those who approve more of others preparing hold more positive attitudes towards disaster preparation. The significant correlation suggests that these measures were tapping similar beliefs, but the weakness of the relationship supports the differentiation of personal norms and attitudes. Further, injunctive norms also positively correlated with attitudes, $r=.292, p<.01$, suggesting that those who thought that others approved of preparation had more positive attitudes. It is also possible that holding positive attitudes towards preparation increases the perception that others also approve, perhaps through an availability heuristic mechanism (Tversky \& Kahneman, 1974).

As expected, descriptive norms correlated positively with injunctive norms, $r=.195, p$ $<.01$. The significant relationship suggests that people who perceive one norm as stronger are likely to perceive the other type of norm as stronger as well. However, the weakness of the relationship supports the differentiation of descriptive and injunctive norms as argued in most recent literature (e.g., Park \& Smith, 2007; Vinnell, Milfont, \& McClure, 2018).

Finally, PBC was positively correlated with both attitudes, $r=.270, p<.01$, and with injunctive norms, $r=.233, p<.01$, suggesting that those who have more positive attitudes 
towards preparation, and those who think others approve of the behaviour, think they have more control over their own preparation. These correlations fit the typical intercorrelations of TPB components proposed by the model (Ajzen, 1991). However, neither PBC nor attitudes correlated with descriptive norms, providing further support to the above finding and the suggestions of previous literature that the two types of normative influence ought to be treated as discrete. 
Table 3.7

Regression of subject and demographic variables on self-report general, survival, and mitigation natural disaster preparation.

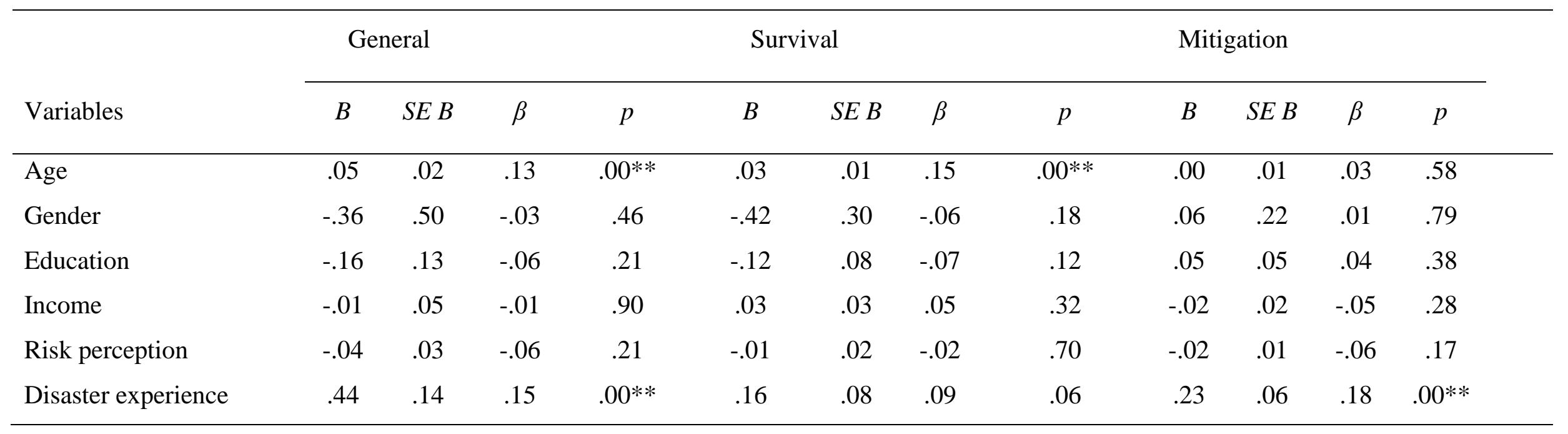


Table 3.7 presents the associations of demographic factors with self-report disaster preparation behaviour. The variables significantly explained approximately $4 \%$ of the variance $\left(R^{2}=.042\right)$ in preparation, $F(6,516)=3.75, p=.001$. Of these variables, only age $(\beta=.134, p$ $=.003)$ and disaster experience $(\beta=.146, p=.001)$ were significantly and positively associated with past behaviour. This demonstrates that the association between some demographic factors and past preparation behaviours, while statistically significant, is weak.

Two subsequent analyses tested for differences in the association between the same demographic factors and the two main types of natural disaster preparation: survival and mitigation (Table 3.7). The overall model for survival preparation was significant, $F(6,516)=$ $3.55, p=.002$, again explaining $4 \%$ of the variance $\left(R^{2}=.04\right)$. Age was significantly and positively associated with this type of preparation $(\beta=.149, p=.001)$. The association of disaster experience was positive but only marginal significant $(\beta=.085, p=.058)$. The overall model for mitigation preparation was also significant, $F(6,515)=3.05, p=.006$, again explaining approximately $4 \%$ of the variance $\left(R^{2}=.035\right)$. The association of disaster experience was again positive and statistically significant $(\beta=.174, p<.001)$, however no other factors, including age, were significantly associated with mitigation preparation. Overall, these findings demonstrate that a weak influence of demographic factors on disaster preparation are limited to effects of disaster experience and age, with those who are older and have more experience being more prepared, consistent with some previous findings (Solberg et al., 2010). 
Table 3.8

Regression of subject and demographic variables on natural disaster preparation intentions.

\begin{tabular}{lcccc}
\hline Variables & $B$ & $S E B$ & $\beta$ & $p$ \\
\hline Age & .02 & .00 & .21 & $.00^{* *}$ \\
Gender & .29 & .11 & .12 & $.01^{*}$ \\
Education & -.09 & .03 & -.15 & $.01^{* *}$ \\
Income & .00 & .01 & .01 & .90 \\
Risk perception & -.01 & .01 & -.04 & .39 \\
Disaster experience & .06 & .03 & .09 & $.05^{*}$ \\
\hline
\end{tabular}

Note. ${ }^{*}=$ significant at the level of $\mathrm{p}<.05 . * *=$ significant at the level of $\mathrm{p}<.01$.

The same demographic factors were tested with the dependent variable of preparation intentions (see Table 3.8). The overall model was significant, $F(6,473)=8.00, p<.001$, explaining approximately $9 \%$ of the variance $\left(R^{2}=.093\right)$, suggesting that demographic factors have a stronger association with behavioural intentions than with behaviour. Age showed the strongest association with intentions $(\beta=.206, p<.001)$, followed by gender $(\beta=.120, p=$ $.01)$, and disaster experience $(\beta=.091, p=.045)$. Further, education was significantly negatively associated with intentions $(\beta=-.149, p=.001)$. These findings suggest that those participants who were older and had more experience of disasters had stronger intentions to prepare in the future, as did those participants with less education. 
Table 3.9

Regression of TPB variables on self-report general, survival, and mitigation disaster preparation, controlling for demographic factors.

\begin{tabular}{|c|c|c|c|c|c|c|c|c|c|c|c|c|}
\hline \multirow[b]{2}{*}{ Variables } & \multicolumn{4}{|c|}{ General } & \multicolumn{4}{|c|}{ Survival } & \multicolumn{4}{|c|}{ Mitigation } \\
\hline & $B$ & $S E B$ & $\beta$ & $p$ & $B$ & $S E B$ & $\beta$ & $p$ & $B$ & $S E B$ & $\beta$ & $p$ \\
\hline \multicolumn{13}{|l|}{ Step One } \\
\hline Age & .02 & .02 & .06 & .43 & .03 & .02 & .13 & .06 & -.02 & .01 & -.11 & .11 \\
\hline Gender & -.10 & .71 & -.01 & .89 & -.24 & .45 & -.03 & .59 & .24 & .32 & .05 & .45 \\
\hline Education & -.08 & .18 & -.03 & .67 & -.03 & .12 & -.02 & .78 & .07 & .08 & .05 & .43 \\
\hline Income & -.05 & .07 & -.05 & .49 & .02 & .05 & .03 & .66 & -.05 & .03 & -.11 & .12 \\
\hline Risk perception & -.07 & .04 & -.11 & .10 & -.03 & .03 & -.07 & .27 & -.02 & .02 & -.08 & .24 \\
\hline Disaster experience & .28 & .19 & .10 & .15 & .05 & .12 & .03 & .67 & .22 & .09 & .17 & $.01 *$ \\
\hline \multicolumn{13}{|l|}{ Step Two } \\
\hline Attitudes & -.08 & .21 & -.03 & .70 & -.10 & .12 & -.05 & .45 & .04 & .09 & .03 & .64 \\
\hline Descriptive norm & .05 & .01 & .25 & $.00 * *$ & .04 & .01 & .25 & $.00 * *$ & .01 & .01 & .15 & $.03 *$ \\
\hline Injunctive norm & .05 & .30 & .01 & .86 & .15 & .19 & .06 & .43 & -.17 & .13 & -.09 & .20 \\
\hline Perceived behavioural control & .86 & .20 & .28 & $.00 * *$ & .49 & .13 & .25 & $.00 * *$ & .27 & .09 & .20 & $.00 * *$ \\
\hline
\end{tabular}

Note. $*$ significant at the level of $p<.05 . * *=$ significant at the level of $p<.01$ 
Table 3.9 presents the association of TPB variables with self-report disaster preparation behaviour, controlling for demographic factors using a hierarchical regression where particular variables are manually entered at each step. Age, gender, education, income, risk perception, and disaster experience were entered in step one and the TPB components (attitudes, descriptive norm, injunctive norm, and perceived behavioural control) entered in the step two. The overall regression model was significant, $F(10,219)=4.42, p<.001$. The TPB components in step two explained an additional $16 \%$ of the variance (change in $R^{2}=.16$ ) above the variance explained by the demographic factors in step one. Of the TPB components, descriptive norms $(\beta=.249, p<.001)$ and $\mathrm{PBC}(\beta=.276, p<.001)$ were positively and significantly associated with preparation. As past preparation is held to be an important predictor of subsequent behaviour (Sniehotta et al., 2005), this finding suggests that descriptive norms and PBC, at least, are likely to be associated with behaviour over time.

As with the demographic factors, two further analyses examined the associations of the TPB variables with the two types of preparation (survival and mitigation: Table 3.9). The overall model for survival preparation was significant, $F(10,219)=4.45, p<.001$, explaining an additional $15 \%$ of the variance above the effect of the demographic factors. As with general preparation, only descriptive norms $(\beta=.254, p<.001)$ and $\operatorname{PBC}(\beta=.247, p<.001)$ were positively and significantly associated with survival preparation. The overall model for mitigation preparation was also significant, $F(10,219)=2.92, p=.002$, explaining approximately $7 \%$ of the variance above that explained by the demographic factors (change in $R^{2}=.065$ ). Again, only descriptive norms $(\beta=.146, p=.032)$ and $\operatorname{PBC}(\beta=.202, p=.003)$ were significantly associated with mitigation preparation. These findings suggest that those who think that other people are preparing for disasters, and who think that they have control over their own preparation, are more likely to have undertaken both survival and mitigation preparation actions.

Notably, these results support that the TPB has potential in predicting disaster preparation. As with the demographic factors, however, it is possible that the patterns of significance would differ when testing both prediction of behaviour and objective measures of behaviour. Finally, the significance of descriptive norms, and lack of significance of injunctive norms, supports the differentiation of the two types of influence within the TPB model as their associations with preparation appear to differ. 
Table 3.10

Regression of TPB variables on self-report natural disaster preparation intentions, controlling for demographic factors.

\begin{tabular}{lcccc}
\hline Variables & $B$ & $S E B$ & $\beta$ & $p$ \\
\hline Step One & & & & \\
\hline Age & .01 & .01 & .10 & .16 \\
Gender & .20 & .16 & .09 & .21 \\
Education & -.11 & .04 & -.17 & $.01 * *$ \\
Income & .02 & .02 & .10 & .17 \\
Risk perception & -.01 & .01 & -.05 & .52 \\
Disaster experience & .07 & .04 & .11 & .13 \\
Step Two & & & & $.02 *$ \\
\hline Attitudes & .12 & .05 & .17 & $.02 *$ \\
Descriptive norm & .00 & .00 & .04 & .15 \\
Injunctive norm & .10 & .07 & .11 & .12 \\
Perceived behavioural control & .07 & .05 & .11 & .01. \\
\hline Note. * significant at the level of $p<.05 . * *=$ significant at the level of $p<.01$ \\
\end{tabular}

The TPB variables explained approximately $7 \%$ of the variance in intentions to prepare $\left(R^{2}\right.$ change $=.065)$ above the effect of demographic factors (see Table 3.10; $F(10,203)=3.16, p=$ $.001)$. In this model, only attitudes were significantly associated with intentions $(\beta=.165, p=$ .018), suggesting that those who have more positive attitudes towards disaster preparation hold stronger intentions to prepare. Taken together with the above findings relating to preparation behaviour, this result demonstrates that all tested TPB variables are significantly associated with either behavioural intentions or self-reported preparation behaviour and provides further support for a more thorough testing of the TPB in the area of disaster preparation. 


\section{Discussion}

This study represents a useful and important first test of the applicability of the TPB to disaster preparation behaviour. While limitations with the method mean that further exploration is needed to draw firm-enough conclusions on which to base an intervention, the findings presented above still prove informative. This section will review the above results in relation to previous literature and predictions; limitations of this study and implications for future studies will then be discussed.

\section{Current Levels of Preparation}

Survival actions were more common than mitigation actions, replicating previous research (e.g., Russell, Goltz, \& Bourque, 1995; Spittal, McClure, Seigert, \& Walkey, 2008), but preparation overall was low. Also consistent with previous research in this context (McClure et al., 2015), cost is only one of the most common barriers for two of the 18 actions: purchasing a water tank and purchasing disaster insurance. It is possible that the responses of cost as a barrier are due to the phrasing of the items, as these two actions are the only ones referring to "purchasing", and other actions, such as strengthening foundations, cost significantly more. These were two of only three actions to be explicitly phrased as a purchase, perhaps increasing the salience of the financial costs of the action. The lack of associations between income and both preparation intentions and behaviour support this suggestion that cost is not the most significant barrier. Frequently, participants indicated that they had not thought about carrying out particular actions, suggesting that education campaigns may need to focus on a wider range of behaviours to ensure that Wellington residents are aware of the many actions they can take.

However, and critical to this body of research, the most common barrier given was getting around to carrying out the action. This suggests that people are aware of what they should be doing to prepare and do not believe they are directly impeded by factors such as cost or difficulty. It is possible that further exploration of people's reasoning for their own behaviour might identify that factors such as cost or difficulty do play a meaningful role in inhibiting preparation. Studies 2 and 3 present one method of exploring in more depth the influences on behavioural decisions to prepare. Possibilities for other methods, in particular qualitative and mixed method approaches, are raised in Chapter 7 (General Discussion).

Reported intentions to prepare were above the midpoint of the scale, suggesting that participants have a desire to carry out preparation actions. This common barrier highlights one of 
the main limitations of the TPB: the intention-behaviour gap, where intentions to carry out a behaviour do not necessarily translate into actual behaviour. Together, these findings imply that a future intervention will need to employ a tactic to motivate behaviour, rather than simply aiming to increase intentions. Potential tactics for bridging this intention-behaviour gap are presented in Chapter 7.

\section{Demographic Factors}

Only three demographic factors were significantly associated with intentions to prepare; participants who are older, have more experience of disasters, and are less educated had stronger intentions to prepare while neither gender nor risk perception played a role. However, these three significant factors only explained $9 \%$ of the variance in intentions. Further, only approximately $4 \%$ of the variance in past behaviour (across general, survival, and mitigation actions) was explained by age and/or experience. These weak associations for some factors and no associations for others supports suggestions from several authors that the influences of demographic factors are inconsistent and have only a minor direct effect on behaviour (e.g., Lindell \& Whitney, 2000; Becker et al., 2015). The sample in this study fairly represented the proportion of Māori and Pasifika in the Wellington Region and over-represented most other minority ethnicities, and so the lack of significant differences in preparedness between ethnicities suggests that any actual differences, if they exist, are small, supporting the intent of this research to identify factors which are broadly relevant across Wellington and New Zealand's cultural context. Further, the limited importance of demographic factors, including culture and experience, suggests that these findings can likely be generalized to other countries, especially those with similar risk profiles and similar cultural dimensions such as individualism.

\section{TPB Components}

Although only attitudes were significantly associated with intentions to prepare, contrary to predictions, this single variable explained $7 \%$ of the variance in intentions when controlling for demographic factors, a similar amount to age, education, and disaster experience combined. This finding suggests that attitudes, at least, are important to consider when examining intentions to prepare for natural disasters. Further, both descriptive norms and perceived behavioural control were positively and significantly associated with past preparation behaviour, demonstrating similar strength associations, although they explained more variance in survival actions (approximately 15\% when controlling for demographic factors) than in mitigation actions 
(approximately 7\% when controlling for demographic factors). While past behaviour is not a formal part of the TPB model (Ajzen, 2011b), it is a useful predictor of subsequent behaviour. Factors which relate to past behaviour are therefore likely to also relate to behaviour over time and ought to be explored further. Across both intentions and behaviour, then, all components of the TPB (attitudes, norms, and control) were found in this study to be relevant to disaster preparation. These findings are consistent with research within the disaster field demonstrating the relevance of each construct individually with preparation, as presented in Chapter 2.

\section{Limitations}

While this study provides interesting and valuable information as to the applicability of the TPB to disaster preparation, the methodology suffered from the inconsistency undermining much of the TPB research field as a whole. The findings of significant pathways between the measures used in this study is encouraging and suggests that further exploration of the model in the disaster context is warranted; however, more rigour will be required in those further explorations. A next step is to test the model again, focusing on the TPB components, addressing the issues inherent in examining multiple behaviours, and addressing the limitations of the sample. Each are discussed below in more detail.

Two-factor TPB. Since the model was first developed, understanding of the components has developed and all three (attitudes, subjective norm, and PBC) are proposed to comprise of two sub-components (Conner et al., 2011). While this study attempted to differentiate a number of the sub-components, and found support for doing so, these differentiations need to be tested in a more thorough, rigorous methodology. Response-efficacy is a close proxy to instrumental attitudes, for example, but does not necessarily capture the same range of judgments that specifically designed measures of instrumental attitude would capture. Similarly, the self-efficacy scale used in this study referred solely to difficulty, whereas other measures used tap into the construct more thoroughly, as well as more clearly delineating the second sub-component, controllability. It is

therefore important to test these sub-components using current best practice in TPB research, to help reduce the inconsistencies which have historically undermined the field (Armitage \& Conner, 2001).

Posing an additional complexity, the components are often treated as a combination of a direct and an indirect measure. For example, while I tested both descriptive and injunctive norms, I did not measure motivation to comply with those norms or strength of identification with the 
norm referent (Ajzen, 2006a), which represent indirect measures of the subjective norm component. Measuring the components in this two-level way will improve our understanding of how they explain intentions and predict behaviour, which will allow us to better understand causal relationships between the components and intentions and behaviour.

Multiple behaviours. As discussed in Chapter 2, the TPB was designed to predict a single behaviour (Ajzen \& Madden, 1986) and although Ajzen, among others, argue that the TPB can be applied to classes of behaviour as long as the items across all scales use the same behavioural wording (Ajzen, 2011a; Manning \& Bettencourt, 2011), doing so requires the wording of the component measures to be vague, as was the case in this study which referred to "preparing for natural disasters" generally. The alternative, for the measures to be duplicated for each behaviour, would result in multiple models and prohibitively long questionnaires. This problem, however, is not unique to the novel context of disaster preparation. The TPB is predominantly used in the health research domain and has been shown to work well when applied to specific behaviours, such as getting vaccinations (e.g., Yang, 2014), exercising (Norman, Conner, \& Bell, 2000), and healthy eating (Tsorbatzoudis, 2005). However, many of the outcomes that are the aim of these studies, such as reducing the risk of certain diseases or improving overall health, are achieved via multiple behaviours. For example, reducing one's blood pressure is achieved through changes in diet, exercise, and stress which represent a number of different behaviours (Peters \& Templin, 2010). Focusing on one of these many behaviours, therefore, is likely not the most effective way to achieve the intended outcome. The same applies to disaster preparation: encouraging people to take a specific action to prepare for a disaster is beneficial, but one action will not make someone “prepared". One study, using a general behavioural "goal" (reducing blood pressure; Peters \& Templin, 2010) and measuring behaviour with a multi-item scale has shown potential predictive utility of the TPB when applied to general behaviours. Similarly, the current study shows that the model is still appropriate to apply in the same way to disaster preparation. However, given the novelty of this area of research, more thorough testing, and in the particular context of disaster preparation, is needed.

Regarding the behaviour measures but separate from the issue of specific or general, the current study treated the presented behaviours as dichotomous, having either been completed or not completed. This presents some issues given that many of the behaviours can be conducted to different extents, and no information was given as to what extent is acceptable. For example, data 
was not collected as to how much water participants had stored. Many of the people who have done this action likely have not stored as much as recommended (20 litres per person, per day, for a week). The same issue applies with stored food. Past advice was to be able to feed yourself for three days, while current forecasts suggest that it will take much longer for help to reach many areas of the region, and so again participants who have stored some food likely have not stored enough. While behaviour change interventions tend to target behaviours which are less commonly done, and so allow for a larger increase, the extent to which these behaviours are carried out can still be increased among those who have started them. Further, that so many participants have made some attempt to carry out these behaviours implies that they might be simple to encourage. Therefore, while storing food and water appear the least necessary to target, they still present a valid option for an intervention.

\section{Conclusion}

This study indicates that people in Wellington are currently under-prepared for a disaster, and that barriers other than cost are playing a prohibitive role. These factors, such as lack of awareness and motivation, can be addressed in behaviour change interventions. While only attitudes were associated with intentions to prepare, both descriptive norms and perceived behavioural control were associated with past behaviour; the evidence found in this study therefore supports further testing of the model in this context. However, several important limitations, such as consistency of the TPB measures and representativeness of the sample, needed to be addressed to provide both firmer support for the applicability of the model to this context and the data required to inform intervention strategies. 


\section{Chapter 4: Study 2}

\section{Introduction}

\section{Overview}

Study 1 demonstrated, as expected, that preparation for natural disasters within the Wellington region is relatively low, supporting further investigation into ways to increase this behaviour. Study 1 also had a secondary purpose of testing the relevance of Theory of Planned Behaviour (TPB) constructs to preparation for natural disasters in a preliminary manner. Evidence from Study 1, as well as previous research (e.g., Johnston et al., 2013McClure, Spittal, Fischer, \& Charleson, 2015), suggests that disaster preparation can be meaningfully increased in the research context of the urban Wellington region, and that the constructs within the TPB are relevant to this behaviour; attitudes were significantly associated with intentions to prepare while descriptive norms and perceived behavioural control (PBC) were both significantly associated with past behaviour.

After establishing the existing level of preparation, the next step in providing a systematic test of the TPB in this research domain is to develop rigorous measures of the TPB constructs. Because the measures used in TPB studies tend to be specific to the particular behaviour and context under examination, it is important to design and then test measures which are consistent with previous research but appropriate for the given study (Sun, Acheampong, Lin, \& Pun, 2015). The purpose of Study 2 is to test an extended TPB measure suitable to test the two-factor, twolevel model discussed in Chapter 2.

Further, this study includes a test of differences between two often-used terms in this field: natural disaster and natural hazard. Particularly with the current global shift within the research field away from using the term "natural disaster" (see e.g., Chmutina \& von Meding, 2019), evidence for any differences of these two phrases on either beliefs and cognitions or associations between constructs will contribute meaningfully to the practical field as well as the scientific literature. Little to no research has considered the impact that these particular terms have on action, or whether the terms are interpreted in the way they are intended (Kelman, 2018).

However, there are several reasons to expect that the terms might be understood differently, particularly based on more general uses of the terms "disaster" and "hazard". While the use of the term "disaster" has shifted to be applicable to a range of relatively minor, everyday negative 
occurrences as explained in the introduction, the term is also still extensively used to refer to devastating natural hazard events. For example, research in the US demonstrates that people's selfperceptions of their knowledge about physical consequences and human behaviour during natural hazard events are extensively influenced by consumption of disaster films and other pop-culture (Quarantelli, 1980). Many of these depictions are pessimistic in nature, perhaps contributing to beliefs that the depicted impacts are unavoidable (Quarantelli, 1980). This genre experienced growth in the 1970s, as well as a resurgence from the mid-1990s which is still largely ongoing (Schroder, 2010). While Schroder argues that many films emphasize control over nature, they also emphasise "destructive powers, frightening scale, and terrifying consequences" (p. 290). Although the evidence is unfortunately limited aside from the research presented by Quarantelli (1980), it is logical to assume that the continuing prevalence and popularity of the disaster pop culture genre means that many people use those depictions to understand the term "disaster". In turn, such an association of the term with those depictions would feasibly reduce perceptions that preparation is a useful and worthwhile endeavour. Research in New Zealand demonstrates, for example, that earthquake damage is seen as less preventable when people are presented with depictions of widespread destruction (McClure et al., 2007; McClure, Allen, \& Walkey, 2001).

In contrast, the term "hazard" is typically used in contexts of identifying potential risks with the deliberate purpose of preventing harm or damage, such as in the context of workplace health and safety. In such contexts, with which most people who have been employed will have at least some experience, the usefulness of prevention and mitigation actions is much more explicit. While the assumptions presented above may be logical, further research to clarify differences in how the terms influence risk-related cognitions and behaviour is valuable. This study presents a quantitative, preliminary test; the potential for a more thorough exploration, including qualitative and mixed method approaches, is discussed later.

As insufficient research exists to formulate hypotheses for these differences, either significant or non-significant findings will prove meaningful to the field. Non-significant findings would suggest that the reasons for the argument against using the term natural disasters do not extend to non-expert audiences, while significant findings would suggest that the terminology used in this field is important to consider when attempting to influence public beliefs and behaviours. As with Study 1, this study uses a sample from the Wellington Region which has experienced natural hazard events historically and recently and has a multi-cultural population. Given the lack 
of effects for ethnicity and only weak effects of experience found in Study 1, any role of these contextual factors should be limited.

\section{Hypotheses}

This study tests the two-factor and two-level TPB (see Figure 4.1). Based on the literature presented in Chapter 2 and the findings of Study 1, I made three hypotheses regarding associations between the independent (TPB cognitions) and dependent variables (intentions and behaviour). The limited research suggesting framing effects of the terms "natural hazard" and "natural disaster" did not provide enough evidence for predictions to be made so these tests were treated as exploratory.

Based on previous research applying the TPB to disaster preparation (Najafi, Ardalan, Akbarisari, Noorbala, \& Elmi, 2017) I expected that both attitudes components (experiential and instrumental), both norms (injunctive and descriptive), and both PBC components (self-efficacy and controllability) would be positively associated with intentions to prepare for natural disasters/hazards. Based on the findings of Study 1, I expected that the attitude components and the PBC components would demonstrate stronger associations than the norm components.

Given the theoretical and methodological refinements in this study, I expected that the TPB variables would explain a larger portion of variance in intentions to prepare than that observed in Study 1 (i.e., 9\%). Finally, I expected that the belief measures would be positively and significantly associated with their relevant cognitions: attitude beliefs would be positively associated with experiential and instrumental attitudes, injunctive and descriptive norm beliefs would be positively associated with injunctive and descriptive norms, and control beliefs would be positively associated with controllability and self-efficacy. 
Chapter 4: Study 2

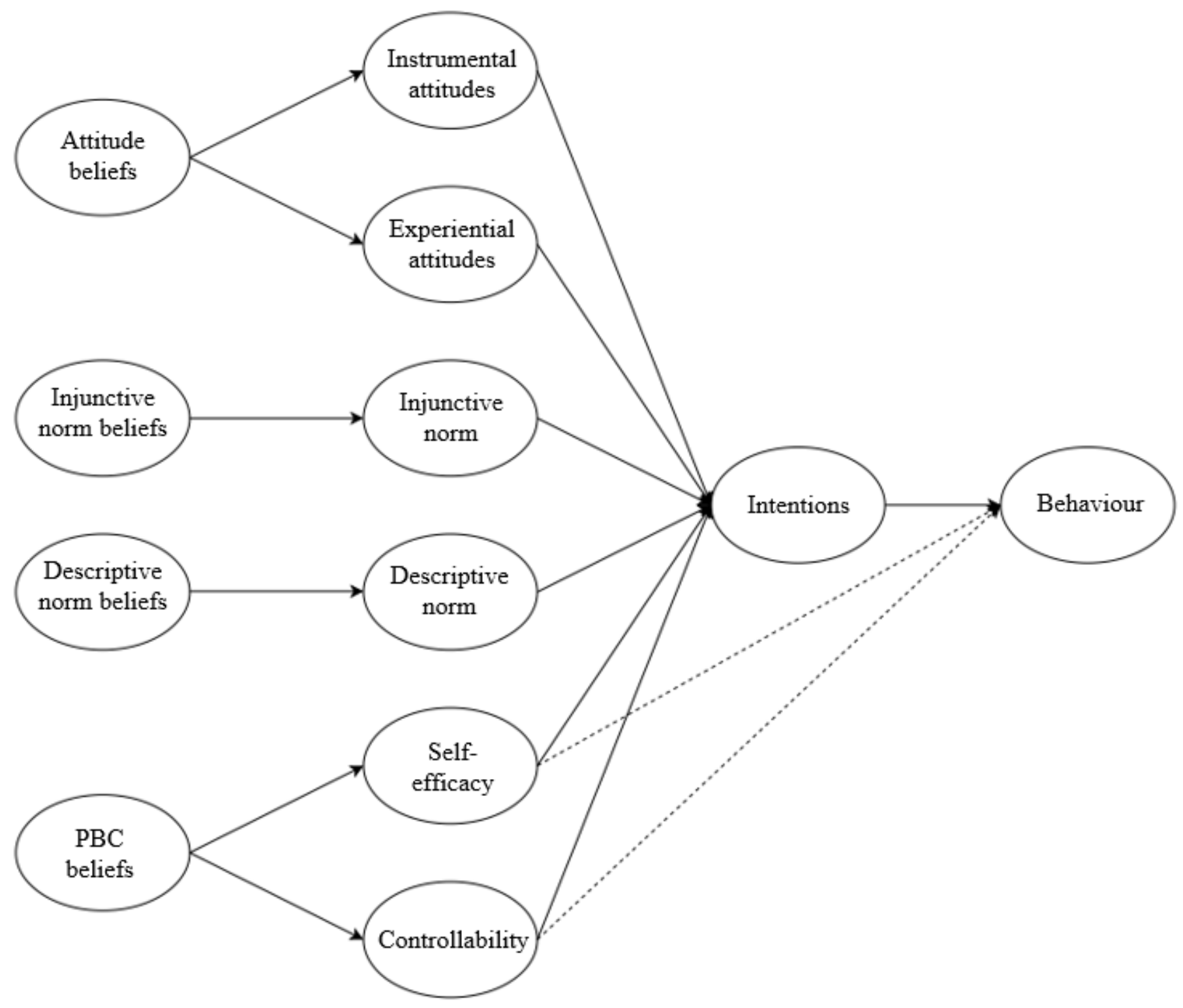

Figure 4.1. The Theory of Planned Behaviour with the cognition components divided into their theorized factors. 


\section{Method}

\section{Design}

Study 2 used a survey design (with an experimental component) to identify relationships between the TPB cognition components, behavioural intentions, and behaviour. The experimental component involved one independent variable (wording) with two levels ("natural disaster" and "natural hazard"), which was manipulated between-subjects.

\section{Participants}

Study 2 used a general population sample from the urban Wellington Region (Wellington City, Hutt Valley, and Porirua). Participants were recruited through social media platforms (Facebook) so were not selected to obtain an even gender split or representative distributions on demographics such as age and ethnicity. Participants were randomly assigned to experimental conditions using the "Randomize" function on Qualtrics. As the purpose of this study was to provide support for the applicability of the TPB in the natural hazard/disaster context, and an initial test of the created measures, this sample was chosen for convenience. Online recruitment is typically faster and cheaper than other methods (Teo, 2013), and therefore suitable for an initial test of theory and measures. The analysis plan to test for effects of the experimental manipulation included comparing structural equation models between the two groups, which requires large sample sizes (Breitsohl, 2018). Past sample sizes gathered using this method (Vinnell, Milfont, \& McClure, 2018, 2019; Study 1 of this thesis) are at the lower limit of being considered large and so no recruitment target was set.

One thousand and ten participants commenced the survey. Given the length of this survey, the drop out-rate was considerable (36\%), with 103 participants not completing more than the first scale, and a further 261 not completing all of the TPB scales. One participant was removed for indicating that they were below the age of consent required by this study, 23 participants indicated they did not live in the urban Wellington Region or did not answer this question, one participant did not understand the definition of natural hazard presented, and a further 11 participants did not respond to this manipulation check. These exclusions resulted in a useable data set of 603 participants: 300 participants in the Disaster condition and 303 in the Hazard condition.

Demographics. Participants' ages ranged from 18 to 73 years old, with a mean of 32.89 years ( $S D=12.70 ; 74$ participants did not provide their age). As with the previous study, there was a distinct gender bias; of the 584 participants who reported their gender, 79 were men (13.5\%), 
502 were women $(86.0 \%)$, and three indicated a non-binary gender $(0.5 \%)$. Of the 583 participants who reported their ethnicity, 427 (73.2\%) identified as New Zealand European/Pākehā, 46 (7.9\%) as Māori, 44 (7.5\%) as European, 22 (3.8\%) as Asian, 15 (2.6\%) as Pasifika, 12 (2.1\%) as North American, $6(1.0 \%)$ as South/Latin American, $3(0.5 \%)$ as African, and $8(1.4 \%)$ as other ${ }^{5}$. Of the 581 participants who indicated their highest level of education, 29 (5.0\%) had not completed secondary school, 167 (28.7\%) had a secondary school qualification, 95 (16.4\%) had a polytechnic or trade certificate, $164(28.2 \%)$ had an undergraduate degree, and $126(21.7 \%)$ had a postgraduate degree (including Masters and Doctorate).

The modal level of annual income before tax was below \$20,000 (26.5\%), with 9.4\% earning between $\$ 20,000$ and $\$ 29,999,6.7 \%$ between $\$ 30,000$ and $\$ 39,999,8.9 \%$ between $\$ 40,000$ and $\$ 49,999,10.3 \%$ between $\$ 50,000$ and $\$ 59,999,5.7 \%$ between $\$ 60,000$ and $\$ 69,999$, $8.0 \%$ between $\$ 70,000$ and $\$ 79,999,3.6 \%$ between $\$ 80,000$ and $\$ 89,999,2.4 \%$ between $\$ 90,000$ and $\$ 99,999$, and $6.5 \%$ earning $\$ 100,000$ or more. Nineteen participants did not respond to the question, and a further 70 responded that they preferred not to report their income. Nearly half (44\%) of respondents indicated that their household costs exceeded their incomes, $35.6 \%$ indicated that their income roughly matched their household costs, and 20.3\% indicated that their income exceeded their household costs.

The majority of respondents were renting (51.8\%), with the next most common housing situations being living in a home that they own (33.3\%) and living in their parents' home (7.0\%). Further, $3.8 \%$ live in a hostel or halls of residence, and $2.7 \%$ in social housing, with the final $1.4 \%$ responding "Other". Most participants who reported housing type lived in a free-standing house $(62.1 \%)$, a unit or flat $(25.2 \%)$, or an apartment $(9.6 \%)$.

\section{Materials}

An online survey included a range of measures adapted primarily from three TPB questionnaire construction guides (Ajzen, 2002; Ajzen, 2013; Francis et al., 2004). All components of the TPB model were measured with four item scales, with items mostly pro-trait; all con-trait items (indicated in Table 4.1) were reverse-coded before analysis. This survey also included the

\footnotetext{
${ }^{5}$ In contrast with Study 1, participants in this study could only indicate the ethnicity with which they most strongly identify, rather than indicating all ethnicities with which they identify. The considerable increase in proportion of New Zealand European/Pākehā, and commensurate decrease in proportion of Māori could be due to participants holding bicultural identities which could be reported previously but here required them to report a single identity.
} 
risk perception scale, disaster experience scale, preparation behaviour question, and demographic questions from Study 1, with minor changes to improve the items. As with Study 1, this survey was pilot tested with a small number of participants $(N=10)$ to ensure clarity and identify any errors with the online survey construction.

This survey had two versions, presented randomly to participants. One version solely referred to "natural disasters" while the other solely referred to "natural hazards". Those participants in the Disaster condition received the same definition of natural disaster as did the participants in Study 1. The participants in the Hazard condition received the following, altered definition:

"This survey will ask a number of questions about natural hazards. For the purpose of this research, a natural hazard is a process or force of nature, such as an earthquake, flood, tsunami, landslide, wildfire, storm, and volcanic eruption, which has the potential to cause negative consequences such as damage, injury, and/or disruption to lifelines (e.g., roads, water, electricity, food and medical supplies)."

As this study manipulated the term used, a question was added to ask whether participants understood the definition. If participants responded "No" they were presented with a simplified version of the definition, and again asked if they understood. If they responded "No" again, they were directed to the end of the survey. Only one participant responded "No" to understanding the first question; this participant also responded "No" to the second definition and so was excluded from the dataset.

For ease of reading, Table 4.1 presents items for the TPB variables from the Disaster condition. In the Hazard condition, the term "natural hazard" or "natural hazard event" was used instead of "natural disaster", depending on the structure of the sentence. The full Disaster condition survey is presented in Appendices 4.A and 4.B. Reliability information for the TPB scales is presented in the results section. 
Table 4.1

TPB component scale items for the Disaster condition.

\begin{tabular}{|c|c|c|c|c|}
\hline Scale & Question & “1” label & “7” label & Adapted from \\
\hline \multirow[t]{4}{*}{ Intentions } & I expect to prepare for a natural disaster & Strongly disagree & Strongly agree & Francis et al. (2004) \\
\hline & I want to prepare for a natural disaster & Strongly disagree & Strongly agree & Francis et al. (2004) \\
\hline & I intend to prepare for a natural disaster & Strongly disagree & Strongly agree & Francis et al. (2004) \\
\hline & I plan to prepare for a natural disaster & Strongly disagree & Strongly agree & Ajzen (2013) \\
\hline \multicolumn{5}{|l|}{ Attitudes } \\
\hline \multirow[t]{4}{*}{ Experiential } & I think that preparing for a natural disaster is & Simple & Complicated & Ajzen \& Madden (1986)* \\
\hline & I think that preparing for a natural disaster is & Boring & Enjoyable & Ajzen \& Madden (1986) \\
\hline & I think that preparing for a natural disaster is & Unpleasant & Pleasant & Ajzen \& Madden (1986) \\
\hline & I think that preparing for a natural disaster is & Rewarding & Unrewarding & Ajzen \& Madden (1986)* \\
\hline \multirow[t]{4}{*}{ Instrumental } & I think that preparing for a natural disaster is & Useless & Useful & Ajzen \& Madden (1986) \\
\hline & I think that preparing for a natural disaster is & Unimportant & Important & Ajzen \& Madden (1986) \\
\hline & I think that preparing for a natural disaster is & Unnecessary & Necessary & Ajzen \& Madden (1986) \\
\hline & I think that preparing for a natural disaster is & Pointless & Valuable & Ajzen \& Madden (1986) \\
\hline \multirow[t]{4}{*}{ Behavioural belief strength } & $\begin{array}{l}\text { Preparing for a natural disaster will result in me and my } \\
\text { home surviving a disaster better }\end{array}$ & Strongly disagree & Strongly agree & Ajzen (2013) \\
\hline & $\begin{array}{l}\text { I will feel like I'm doing something life-saving if I } \\
\text { prepare for a natural disaster }\end{array}$ & Strongly disagree & Strongly agree & Francis et al. (2004) \\
\hline & It is complicated to prepare for a natural disaster & Strongly disagree & Strongly agree & Francis et al. (2004) \\
\hline & It is expensive to prepare for a natural disaster & Strongly disagree & Strongly agree & Francis et al. (2004) \\
\hline Outcome evaluation & Getting through a natural disaster better is: & Extremely bad & Extremely good & Ajzen (2013) \\
\hline
\end{tabular}


Doing something life-saving is:

Doing something complicated is:

Doing something expensive is:

Subjective norms

Direct

Normative belief strength prepare for a natural disaster disaster natural disaster natural disaster prepared for a natural disaster disaster.
Most people who are important to me think that

It is expected of me that I prepare for a natural disaster

I feel under social pressure to prepare for a natural Strongly disagree

People who are important to me want me to prepare for a Strongly disagree

Most people who are important to me have prepared for a

Most people like me have prepared for a natural disaster Strongly disagree The people in my life whose opinions I value have Strongly disagree

How many of the people who are important to you do you None think have prepared for a natural disaster?

Most of my friends and family have prepared for a natural False

Most of my neighbours have prepared for a natural False disaster.

Most Wellingtonians have prepared for a natural disaster. False
Extremely good

Extremely good

Extremely good

I should not

Strongly agree

Strongly agree

Strongly agree

Strongly agree

Ajzen (2002)

Strongly agree

Ajzen (2002)

Strongly agree

Ajzen (2002)

All

True

Ajzen (2013)

True

Ajzen (2013)

True

Ajzen (2013) 
Most New Zealanders have prepared for a natural False disaster.

When it comes to preparing for a natural disaster, how Very much much do you want to be like your friends and family?

Identification with referent When it comes to preparing for a natural disaster, how much do you want to be like your neighbours?

When it comes to preparing for a natural disaster, how Very much much do you want to be like other Wellingtonians?

When it comes to preparing for a natural disaster, how Very much much do you want to be like other New Zealanders?

Injunctive norms

Direct

Most people who are important to approve of my Strongly disagree preparing for a natural disaster

Most people like me approve of my preparing for a Strongly disagree natural disaster

The people in my life whose opinions I value approve of Strongly disagree my preparing for a natural disaster

Among the people who are important to you, how much No agreement agreement would there be that preparing for a natural disaster is a good thing to do?

Normative belief strength
Most of my friends and family approve of my preparing Strongly disagree for a natural disaster.
True

Ajzen (2013)

Not at all

Ajzen (2013)

Not at all

Ajzen (2013)

Not at all

Ajzen (2013)

Not at all

Ajzen (2013)

Strongly agree

Ajzen (2002)

Strongly agree

Ajzen (2002)

Strongly agree

Ajzen (2002)

A great deal of White et al. (2009) agreement

Strongly agree

Ajzen (2013) 
Most of my neighbours approve of my preparing for a Strongly disagree natural disaster.

Most Wellingtonians approve of my preparing for a Strongly disagree natural disaster.

Most New Zealanders approve of my preparing for a Strongly disagree natural disaster.

Motivation to comply

When it comes to preparing for a natural disaster, I want to do what my friends and family think I should do.

When it comes to preparing for a natural disaster, I want Strongly disagree to do what my neighbours think I should do.

When it comes to preparing for a natural disaster, I want Strongly disagree to do what other Wellingtonians think I should do.

When it comes to preparing for a natural disaster, I want Strongly disagree to do what other New Zealanders think I should do.

\section{PBC}

Self-efficacy

Controllability
I am confident that I can prepare for a natural disaster

If I wanted to, I could prepare for a natural disaster

For me to prepare for a natural disaster is:

For me to prepare for a natural disaster is:

my control

My preparing for a natural disaster is up to me
Strongly agree

Ajzen (2013)

Strongly agree

Ajzen (2013)

Strongly agree

Ajzen (2013)

Strongly agree

Ajzen (2013)

Strongly agree

Ajzen (2013)

Strongly agree

Ajzen (2013)

Strongly agree

Ajzen (2013)

Strongly disagree

Strongly agree

Francis et al. (2004)

Strongly disagree

Strongly agree

Ajzen (2002)

Extremely easy

Extremely

Ajzen (2002)

difficult

Definitely impossible

Definitely

possible

Strongly agree

Francis et al. (2004)*

Strongly disagree

Strongly agree

Ajzen (2013) 
It is mostly up to me whether or not I prepare for a natural Strongly disagree disaster

How much control do you believe you have over No control preparing for a natural disaster?

Control belief strength

Power of control factors
In the next month, I will have the money to prepare for a Extremely unlikely natural disaster

In the next month, I will think about preparing for a Extremely unlikely natural disaster

In the next month, I will have the time to prepare for a Extremely unlikely natural disaster

In the next month, I will make the effort to prepare for a Extremely unlikely natural disaster

Having enough money would make me more likely to Strongly disagree prepare for a natural disaster

Thinking about preparing would make me more likely to Strongly disagree prepare for a natural disaster

Having the time would make me more likely to prepare Strongly disagree for a natural disaster

Making the effort to prepare for a natural disaster would Strongly disagree make me more likely to prepare

$\begin{array}{ll}\text { Strongly agree } & \text { Ajzen (2013) } \\ \text { Complete control } & \text { Ajzen (2002) } \\ \text { Extremely likely } & \text { Ajzen (2013) } \\ \text { Extremely likely } & \text { Ajzen (2013) } \\ \text { Extremely likely } & \text { Ajzen (2013) } \\ \text { Extremely likely } & \text { Ajzen (2013) } \\ \text { Strongly agree } & \text { Ajzen (2013) } \\ \text { Strongly agree } & \text { Ajzen (2013) } \\ \text { Strongly agree } & \text { Ajzen (2013) } \\ \text { Strongly agree } & \text { Ajzen (2013) }\end{array}$

Strongly agree 
Risk perception. This six-item measure, adapted from Terpstra and Lindell (2012), was unchanged from Study 1. As with Study 1 (Cronbach's $\alpha=.86$ ), this scale demonstrated acceptable internal reliability $(\alpha=.86)$, which would not be improved by removing any of the items.

Disaster experience. This three-item measure, adapted from McClure, Willis, Johnston, and Recker (2011) was also unchanged from Study 1, and once again demonstrated acceptable internal reliability for a short scale $(\alpha=.64)$, an increase from Study $1(\alpha=.57)$. Corrected itemtotal correlations were within an acceptable range (.385 to .537$)$

Disaster preparation. A question measuring past behaviour was adapted from Ajzen (2006b). The question read: "In the past three months, I have prepared for a natural disaster." Participants responded on a 7-point Likert-type scale, ranging from 1 (False) to 7 (True). Participants then indicated which of 18 behaviours they had performed. These behaviours were the same as those in Study 1 except that one behaviour, volunteering time to help the community in the event of a disaster, was changed to reflect the recent shift in how Community Emergency Hubs function. As summarised in Chapter 1, Civil Defence shelters, which used to stock emergency supplies, have recently been changed to Hubs where community members can gather and coordinate local response efforts. The updated item read: "Identify my local Community Emergency Hub so I can volunteer in the event of a natural disaster”.

Behaviour. Finally, participants were asked "Would you like to receive an Earthquake Planning Guide?" Those who answered "Yes" were provided a link to a downloadable step-bystep guide to preparing for an earthquake (Wellington Region Emergency Management Office, n.d.). Those who answered "No" were directed to the end of the survey. Some researchers argue that information seeking is not a good proxy for preparation because people can be motivated to avoid risk information yet still prepare (Kahlor, Olson, Markman, \& Wang, 2018) and risk information can lower concern about hazards (Ballantyne, Paton, Johnston, Kozuch, \& Daly, 2000). However, such a measure is commonly used in this way, the information offered in this study is about how to prepare rather than risk, and information has been shown to increase preparedness (Bourque, 2013; Perry \& Lindell, 2008).

Data treatment. Some items were reverse-coded so that all scale values ranged from low, indicating weak or negative responses, to high, indicating strong or positive responses. These items are indicated with asterisks in Table 4.1. As each belief is measured with two scales comprising four items each, the paired items across the two scales are multiplied to create four values per 
participant per belief (Francis et al., 2004). These values are used to create a mean belief score for each participant. Reliability analyses and $t$-tests were conducted using SPSS Version 25. Model fit and structural equation modelling was conducted using Mplus Version 8.1 (Muthén \& Muthén, 1998-2017).

\section{Procedure}

The study was delivered online using the survey platform Qualtrics between August $3^{\text {rd }}$ and August 22 $2^{\text {nd }}, 2018$. This study used a procedure identical to that in Study 1, with three important exceptions. First, this survey included two experimental conditions to test effects of referring to natural disasters versus natural hazards. Second, all participants were presented with all questions. Third, before commencing the survey, participants were asked whether they were willing to have their contact details added to a secure mailing list for potential, future studies. Completion took approximately 15 minutes and participants were thanked with the opportunity to enter a prize draw for one of three $\$ 100$ supermarket gift cards by following a link to a second Qualtrics survey to enter their details, meaning that their identifying data could not be linked to their questionnaire responses. This same link was used for those wishing to join the mailing list. This study was approved by the School of Psychology Human Ethics Committee under the delegated authority of the Victoria University of Wellington Human Ethics Committee (approved: $23^{\text {rd }}$ July 2018; reference number: 0000026244).

\section{Results}

\section{Descriptive Statistics}

Reliability. Table 4.2 presents the Cronbach's alphas for all TPB scales, split by condition. All scales demonstrated acceptable to good internal reliability, with the exception of experiential attitudes and attitude beliefs. Several of the corrected item-total correlations were low for these two scales as well. However, the alpha value would not be increased by excluding any of the individual items. These scales may therefore require future refinement but were deemed acceptable for the research purpose of Study 2. 
Table 4.2

Cronbach's alpha reliability scores for all TPB scales split by condition.

\begin{tabular}{|c|c|c|c|c|c|c|c|c|c|c|c|}
\hline & & Disaster & & & & & Hazard & & & & \\
\hline Cognition & Factor & Alpha & Item 1 & Item 2 & Item 3 & Item 4 & Alpha & Item 1 & Item 2 & Item 3 & Item 4 \\
\hline \multirow[t]{3}{*}{ Attitudes } & Experiential & .558 & .233 & .445 & .452 & .286 & .658 & .234 & .597 & .570 & .420 \\
\hline & Instrumental & .918 & .764 & .838 & .838 & .830 & .900 & .735 & .810 & .802 & .803 \\
\hline & Beliefs & .552 & .432 & .434 & .295 & .214 & .478 & .346 & .412 & .175 & .189 \\
\hline \multirow[t]{2}{*}{ Injunctive norms } & Direct & .844 & .816 & .723 & .772 & .431 & .851 & .770 & .719 & .766 & .523 \\
\hline & Beliefs & .849 & .577 & .640 & .786 & .774 & .874 & .637 & .723 & .765 & .803 \\
\hline \multirow[t]{2}{*}{ Descriptive norms } & Direct & .868 & .831 & .602 & .814 & .641 & .872 & .819 & .635 & .760 & .696 \\
\hline & Beliefs & .819 & .521 & .638 & .758 & .736 & .789 & .457 & .619 & .716 & .661 \\
\hline \multirow[t]{4}{*}{$\mathrm{PBC}$} & Self-efficacy & .782 & .694 & .668 & .438 & .566 & .784 & .631 & .620 & .512 & .610 \\
\hline & Controllability & .707 & .426 & .549 & .571 & .459 & .719 & .502 & .535 & .561 & .488 \\
\hline & Beliefs & .832 & .552 & .689 & .653 & .752 & .836 & .585 & .681 & .649 & .759 \\
\hline & Intentions & .849 & .764 & .467 & .780 & .761 & .863 & .755 & .525 & .778 & .798 \\
\hline
\end{tabular}

Note. "Item" refers to corrected item-total correlations for each question in the scale, following the order presented in Table 4.1. 
TPB scores. Table 4.3 presents the means and standard deviations for all TPB scales, split by condition as well as the range and difference from the midpoint of the scale.

Table 4.3

Descriptive statistics for all TPB scales, split by condition.

\begin{tabular}{|c|c|c|c|c|c|c|}
\hline Disaster & & & & & & \\
\hline Cognition & Factor & $N$ & Mean & $S D$ & Range & Difference from midpoint \\
\hline Attitude & Experiential & 287 & 4.00 & 1.09 & 1 to 7 & $t(286)=.06, p=.95, d=0.00$ \\
\hline & Instrumental & 288 & 6.39 & 1.08 & 1 to 7 & $t(287)=37.70, p<.001, d=2.21$ \\
\hline & Beliefs & 297 & 25.60 & 6.62 & 8 to 42.25 & $t(296)=1.57, p=.12, d=0.09$ \\
\hline Injunctive & Direct & 299 & 5.62 & 1.16 & 1 to 7 & $t(298)=24.23, p<.001, d=1.40$ \\
\hline & Beliefs & 298 & 20.83 & 10.6 & 1 to 49 & $t(297)=-6.83, p<.001, d=0.39$ \\
\hline Descriptive & Direct & 299 & 3.81 & 1.43 & 1 to 7 & $t(298)=-2.36, p<.05, d=0.13$ \\
\hline & Beliefs & 297 & 13.21 & 6.94 & 1 to 49 & $t(296)=-29.25, p<.001, d=1.70$ \\
\hline PBC & Self-efficacy & 300 & 5.42 & 1.13 & 1 to 7 & $t(299)=21.90, p<.001, d=1.26$ \\
\hline & Controllability & 300 & 5.83 & 1.05 & 1 to 7 & $t(299)=30.37, p<.001, d=1.74$ \\
\hline & Beliefs & 299 & 22.46 & 11.12 & 1 to 49 & $t(298)=-3.96, p<.001, d=0.23$ \\
\hline Intentions & & 294 & 5.29 & 1.29 & 1.50 to 7 & $t(293)=17.07, p<.001, d=1.00$ \\
\hline Hazard & & & & & & \\
\hline Attitude & Experiential & 282 & 4.02 & 1.07 & 1 to 7 & $t(281)=.30, p=.767, d=0.02$ \\
\hline & Instrumental & 284 & 6.25 & 1.12 & 1 to 7 & $t(283)=33.96, p<.001, d=2.01$ \\
\hline & Beliefs & 303 & 24.89 & 6.45 & 4.25 to 43.75 & $t(302)=-.31, p=.76, d=0.02$ \\
\hline Injunctive & Direct & 303 & 5.43 & 1.20 & 1.75 to 7 & $t(302)=20.73, p<.001, d=1.19$ \\
\hline & Beliefs & 303 & 20.52 & 11.04 & 1 to 49 & $t(302)=-7.07, p<.001, d=0.41$ \\
\hline Descriptive & Direct & 303 & 3.80 & 1.40 & 1 to 7 & $t(302)=-2.49, p<.05, d=0.14$ \\
\hline & Beliefs & 303 & 13.39 & 7.04 & 1 to 43.75 & $t(302)=-28.70, p<.001, d=1.65$ \\
\hline PBC & Self-efficacy & 303 & 5.34 & 1.15 & 1 to 7 & $t(302)=20.35, p<.001, d=1.17$ \\
\hline & Controllability & 303 & 5.85 & 1.04 & 1.75 to 7 & $t(302)=30.89, p<.001, d=1.78$ \\
\hline & Beliefs & 303 & 21.89 & 11.06 & 1 to 49 & $t(302)=-4.89, p<.001, d=0.28$ \\
\hline Intentions & & 301 & 5.20 & 1.38 & 1 to 7 & $t(300)=15.19, p<.001, d=0.87$ \\
\hline
\end{tabular}

Note. All direct measures had a possible range of 1 to 7 , with a midpoint of 4 . All indirect measures had a possible range of 1 to 49, with a midpoint of 25 . 


\section{Inferential Statistics}

Between-group comparisons. The Hazard and Disaster groups did not significantly differ on any of the demographic variables or on self-reports of past behaviour. Independent samples $t$ tests were used to test for differences between the two groups on mean scores for the TPB scales. The two groups only significantly differed on the direct measure of injunctive norm, which showed a significantly higher mean in the Disaster condition than in the Hazard condition, $t(600)=2.03$, $p<.05, d=.17$. This effect was driven by a difference between the two groups in the second item on this scale ("Most people like me approve of my preparing for a natural disaster / hazard"), with a higher mean in the Disaster condition $(M=5.43, S D=1.42)$ compared to the Hazard condition $(M=5.18, S D=1.37 ; t(598)=2.14, p<.05, d=0.18)$. While this is a weak effect, it demonstrates that participants perceived a stronger norm of approval among people like themselves for natural disaster preparation than for natural hazard preparation. This item was the only one to use similar others as a referent group, while the rest of the items referred to people who are important to the participant as the referent group. Implications of this finding are discussed later.

Except for this difference in the direct injunctive norm measure, driven by a different mean for a single item, none of the means for the TPB scales differed between the two groups. Further, a chi-squared test showed that the ratio of participants in the Disaster condition who did and did not request additional information about how to prepare for an earthquake (172 and 108, respectively) did not differ from the ratio in the Hazard condition (179 and 110, respectively; $p=$ .90).

This lack of significant differences between the Hazard and Disaster conditions suggests that, on the whole, people have similar attitudes, perceptions of norms, perceptions of control, and intentions to prepare for natural disasters and natural hazards. Although this comparison of means is informative, given the structural nature of the theory being tested, it was also pertinent to examine the associations between the variables in the TPB model between the two samples.

Confirmatory factor analysis. The first step in the process of latent path comparison is to establish adequately fitting baseline models. An advantage of this process is that this first step requires confirmatory factor analysis (CFA) of the individual scales, which helps to identify any issues with the items designed in this study, and a CFA of the overall TPB model, which demonstrates whether the theorized paths are relevant within this sample and behavioural context. 
For these analyses, the two conditions are treated separately as invariance has not yet been established.

Disaster condition construct CFAs. Table 4.4 presents the results for individual CFAs on the TPB constructs, including the two-factor, two-level distinction, within the Disaster condition. Adequate construct fit followed the guidelines suggested by Vandenberg and Lance (2000), where the model chi-squared statistic divided by the degrees of freedom should give a ratio below three, CFI should be equal to or greater than .90, and RMSEA and SRMR should be less than .08. Several constructs required a single modification, allowing two items in the scale to correlate, in order to achieve adequate model fit looking holistically across fit indices. These modifications are presented in Table 4.4. Only the two direct measures of attitudes did not achieve acceptable model fit after a first modification according to one or two of the fit indices. In neither case would a further modification have meaningfully improved model fit. In line with the lower reliabilities for these scales, this finding of less-ideal model fit for the attitude factors further suggests that refinement to these measures could be made.

Disaster condition baseline model CFA. Table 4.5 presents the fit indices for the disaster baseline model, which indicates how well the data fit with an imposed TPB model. 
Table 4.4

Results of Confirmatory Factor Analyses for individual TPB constructs within the Disaster condition.

\begin{tabular}{|c|c|c|c|c|c|c|c|c|}
\hline Cognition & Factor & $\chi^{2}$ & $\mathrm{df}$ & $p$ value & Ratio & $\mathrm{CFI}$ & RMSEA & SRMR \\
\hline \multirow[t]{6}{*}{ Attitude } & Experiential & 33.60 & 2 & .000 & 16.80 & .893 & .231 & .083 \\
\hline & Item 1 with 4 & 4.28 & 1 & .039 & 4.28 & .989 & .105 & .032 \\
\hline & Instrumental & 32.22 & 2 & .000 & 16.11 & .966 & .228 & .023 \\
\hline & Item 2 on 4 & 3.60 & 1 & .058 & 3.60 & .997 & .094 & .009 \\
\hline & Beliefs & 88.58 & 2 & .000 & 44.29 & .565 & .380 & .133 \\
\hline & Item 1 with 2 & 0.10 & 1 & .747 & 0.10 & 1.00 & .000 & .003 \\
\hline \multirow[t]{11}{*}{ Norms } & Injunctive & 14.08 & 2 & .001 & 7.04 & .980 & .142 & .025 \\
\hline & Item 4 on 1 & 0.00 & 1 & .977 & 0.00 & 1.00 & .000 & .000 \\
\hline & Injunctive beliefs & 15.31 & 2 & .001 & 7.66 & .977 & .149 & .023 \\
\hline & Item 1 on 4 & 0.95 & 1 & .331 & 0.95 & 1.00 & .000 & .007 \\
\hline & Descriptive & 1.41 & 2 & .494 & 0.71 & 1.00 & .000 & .008 \\
\hline & Descriptive beliefs & 0.26 & 2 & .877 & 0.13 & 1.00 & .000 & .003 \\
\hline & Self-efficacy & 4.60 & 2 & .100 & 4.30 & .993 & .066 & .016 \\
\hline & Controllability & 16.38 & 2 & .000 & 8.19 & .945 & .155 & .048 \\
\hline & Item 1 with 4 & 0.09 & 1 & .759 & 0.09 & 1.00 & .000 & .002 \\
\hline & Beliefs & 29.92 & 2 & .000 & 14.96 & .945 & .216 & .047 \\
\hline & Item 4 on 2 & 2.77 & 1 & .096 & 2.77 & .996 & .077 & .009 \\
\hline Intentions & & 0.08 & 2 & .963 & 0.034 & 1.00 & .000 & .002 \\
\hline
\end{tabular}


Table 4.5

Baseline model fit for the full TPB in the Disaster condition.

\begin{tabular}{|c|c|c|c|c|c|c|c|}
\hline Model & $\chi^{2}$ & df & $p$ value & Ratio & RMSEA & $\mathrm{CFI}$ & SRMR \\
\hline Baseline model & 1686.51 & 853 & .000 & 1.98 & .058 & .862 & .098 \\
\hline 1 st modification & 1721.68 & 852 & .000 & 2.02 & .058 & .858 & .096 \\
\hline \multicolumn{8}{|c|}{ Descriptive norm on intentions } \\
\hline 2nd modification & 1652.42 & 851 & .000 & 1.94 & .056 & .869 & .095 \\
\hline \multicolumn{8}{|c|}{ Attitude beliefs item 3 with item 4} \\
\hline 3rd modification & 1627.55 & 850 & .000 & 1.92 & .055 & .873 & .089 \\
\hline \multicolumn{8}{|c|}{ Descriptive norm with intentions } \\
\hline 4th modification & 1560.87 & 849 & .000 & 1.84 & .053 & .884 & .085 \\
\hline \multicolumn{8}{|c|}{ Descriptive norm (mean) with item 1} \\
\hline 5th modification & 1490.22 & 848 & .000 & 1.76 & .050 & .895 & .081 \\
\hline \multicolumn{8}{|c|}{ Descriptive norm by beliefs item 1} \\
\hline 6th modification & 1442.98 & 847 & .000 & 1.70 & .048 & .903 & .080 \\
\hline
\end{tabular}


As with the individual construct CFAs, the model was modified until it achieved adequate fit. For these analyses, this meant meeting the threshold for RMSEA, CFI, and SRMR, as authors reporting baseline model fit typically do not need chi-square results to show good fit (e.g., Breitsohl, 2018) given the sensitivity of chi-square to sample size. The baseline model of the TPB in the Disaster condition required six modifications to reach adequate model fit across the other three indices, presented in Table 4.5.

Hazard condition construct CFAs. Table 4.6 presents the results for individual CFAs on the TPB constructs, including the two-factor, two-level distinction, within the Hazard condition. As with the Disaster condition, several constructs required a single modification to the model in order to achieve acceptable model fit. These modifications are presented in Table 4.6. Fewer constructs required such modification in the Hazard condition compared to the Disaster condition, suggesting that within this Hazard condition the data fit the theoretical constructs better.

Hazard condition baseline model CFA. Table 4.7 presents the fit indices for the Hazard condition baseline model. As with the disaster baseline model, the Hazard condition model was modified until it achieved acceptable model fit across the three indices of RMSEA, CFI, and SRMR. The baseline model for the Hazard condition required five modifications which are presented in Table 4.7. Similar to the findings of fewer individual construct modifications, the baseline model for the Hazard condition required one less modification than the model for the Disaster condition, suggesting that the structure of the data more closely fit with the imposed theoretical structure. However, this fit is still not particularly strong. 
Table 4.6

Results of Confirmatory Factor Analyses for individual TPB constructs within the Hazard condition.

\begin{tabular}{|c|c|c|c|c|c|c|c|c|}
\hline Cognition & Factor & $\chi^{2}$ & $\mathrm{df}$ & $p$ value & Ratio & CFI & RMSEA & SRMR \\
\hline \multirow[t]{5}{*}{ Attitude } & Experiential & 4.21 & 2 & .122 & 2.11 & .991 & .061 & .027 \\
\hline & Instrumental & 85.50 & 2 & .000 & 42.75 & .897 & .379 & .045 \\
\hline & Item 1 on 2 & 3.55 & 1 & .059 & 3.55 & .997 & .094 & .007 \\
\hline & Beliefs & 65.50 & 2 & .000 & 32.75 & .629 & .324 & .117 \\
\hline & Item 1 with 2 & 1.39 & 1 & .239 & 1.39 & .998 & .036 & .020 \\
\hline \multirow[t]{10}{*}{ Norms } & Injunctive & 1.59 & 2 & .452 & 0.80 & 1.00 & .000 & .008 \\
\hline & Injunctive beliefs & 0.18 & 2 & .912 & 0.09 & 1.00 & .000 & .003 \\
\hline & Descriptive & 3.42 & 2 & .181 & 1.71 & .998 & .048 & .011 \\
\hline & Descriptive beliefs & 1.88 & 2 & .391 & 0.94 & 1.00 & .000 & .012 \\
\hline & Self-efficacy & 14.78 & 2 & .001 & 7.39 & .963 & .145 & .033 \\
\hline & Item 2 on 4 & 0.57 & 1 & .449 & 0.57 & 1.00 & .000 & .006 \\
\hline & Controllability & 24.76 & 2 & .000 & 12.38 & .917 & .194 & .054 \\
\hline & Item 3 on 2 & 0.35 & 1 & .557 & 0.35 & 1.00 & .000 & .004 \\
\hline & Beliefs & 47.81 & 2 & .000 & 23.91 & .917 & .275 & .061 \\
\hline & Item 2 on 4 & 1.72 & 1 & .189 & 1.72 & .999 & .049 & .007 \\
\hline Intentions & & 5.90 & 2 & .052 & 2.95 & .994 & .080 & .017 \\
\hline
\end{tabular}


Table 4.7

Baseline model fit for the full TPB in the Hazard condition.

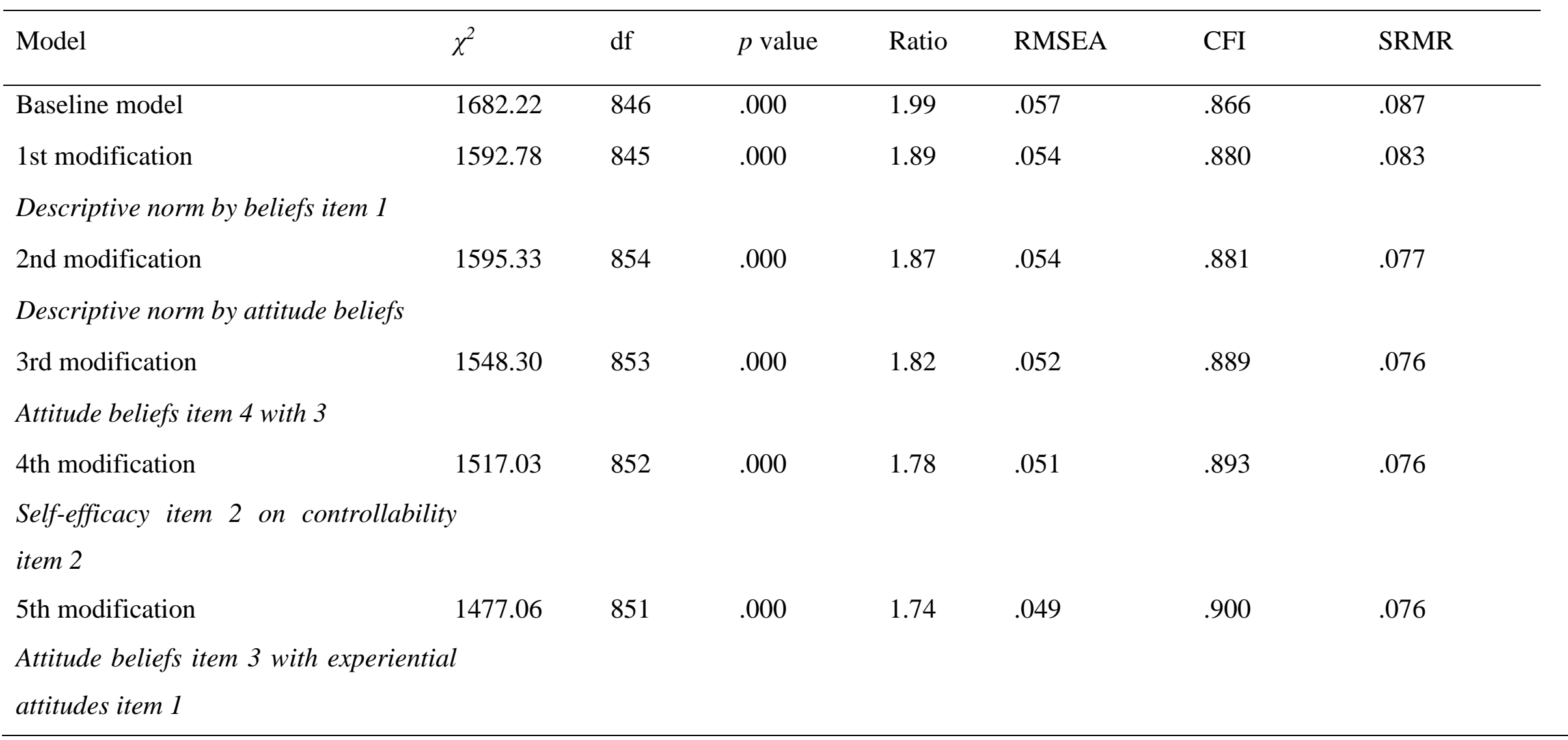


Configural invariance. The next step in comparing structural equation models between groups is to test configural invariance; that is, if the underlying structure of the models vary significantly or not across groups (e.g., Milfont \& Fischer, 2010). After establishing configural invariance, the next steps are to demonstrate metric and scalar invariance, which test whether factor loadings and intercepts vary between the two groups. However, given that the items differed in wording between the two groups, the means and intercepts are not directly comparable in this way, so these forms of invariance were not tested (Gregorich, 2007).

The full TPB model for the Disaster condition (see Figure 4.2), including the belief constructs and cognition subcomponents, was compared to the same model for the Hazard condition (Figure 4.3) to establish if the fit of the data to the imposed model varied between the two groups. As with the baseline model CFAs, the model chi-squared statistic is typically reported but does not impact whether configural invariance is said to be demonstrated or not (e.g., Breitsohl, 2018). Our two models, therefore, demonstrated adequate model fit ( $\chi^{2}$ (1763) $=3003.194, p<.001 ; \mathrm{RMSEA}=.048, \mathrm{CFI}=.900, \mathrm{SRMR}=.081)$, suggesting that there is little variance in the overall structure between the groups. Metric and scalar invariance could not be established, and therefore it is inappropriate to run a full multigroup SEM, which demonstrates change in fit between a model where factor loadings and intercepts are constrained to be equal between the two groups and a model where both groups are allowed to vary freely. However, the finding of configural invariance supports a non-statistical comparison of models for the two groups in a simple multigroup SEM where the same model is tested for in two groups simultaneously. This means that the resulting model outputs for the two groups can be compared visually for differences in the significance of paths and amount of variance in the dependent variable explained. Figure 4.2 presents the SEM for the Disaster condition and Figure 4.3 presents the SEM for the Hazard condition, using the two-level, two-factor TPB. 


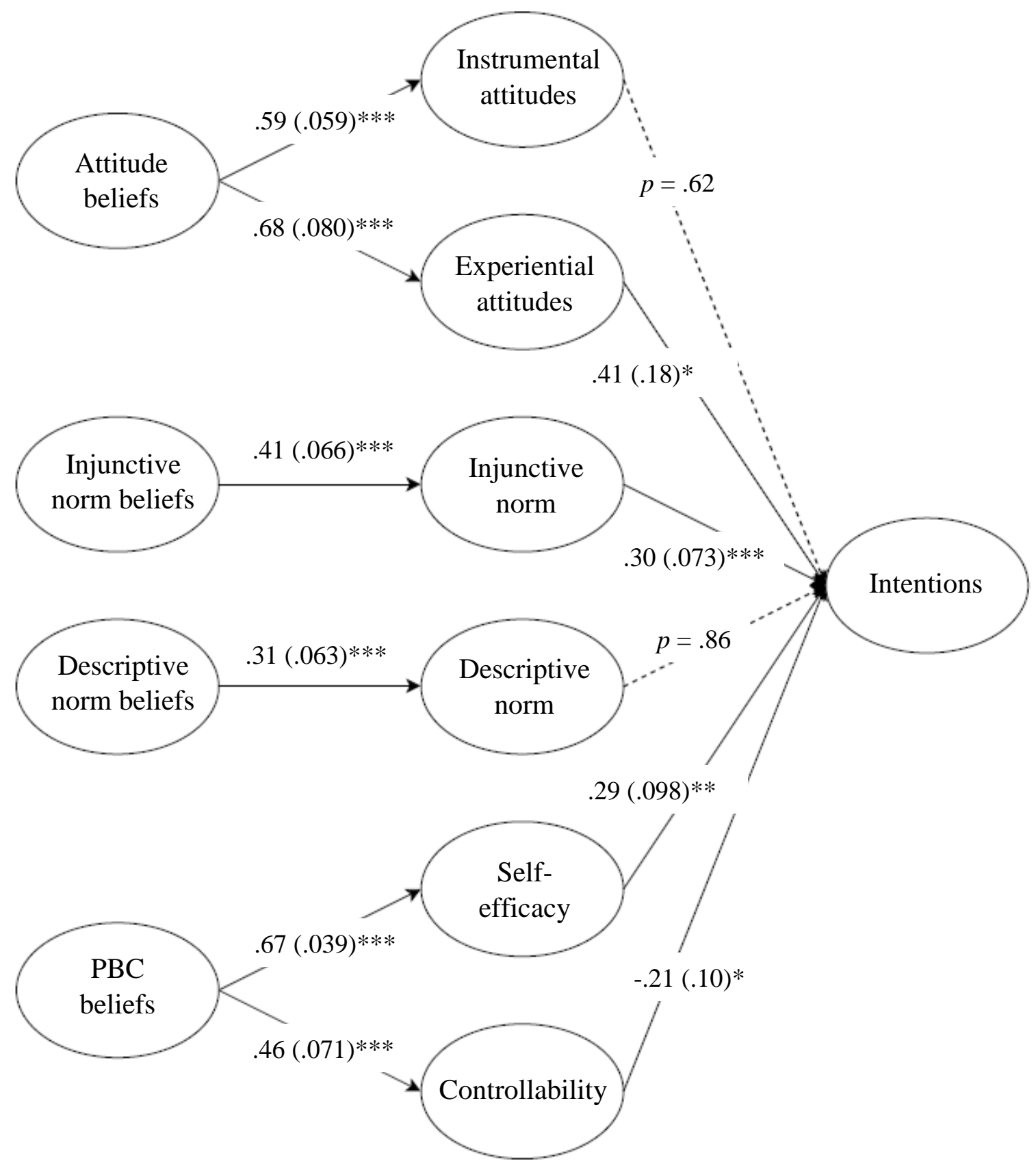

Figure 4.2. Structural equation model for the Disaster condition. Dotted line indicates a nonsignificant path. For these paths, the associated numbers are the $p$ values. For significant paths, the numbers represent the standardized estimates, with the standard errors in parentheses. $R^{2}$ for intentions $=.426$. Adjusted $R^{2}$ for intentions $=.414^{6} . * p<.05, * * p<.01, * * * p<.001$.

\footnotetext{
${ }^{6}$ Mplus does not calculate adjusted $R^{2}$ values, so the following formula was used: AdjRSQ = RSQ ((1 - RSQ) * numIVs)/(n - numIVs -1).
} 


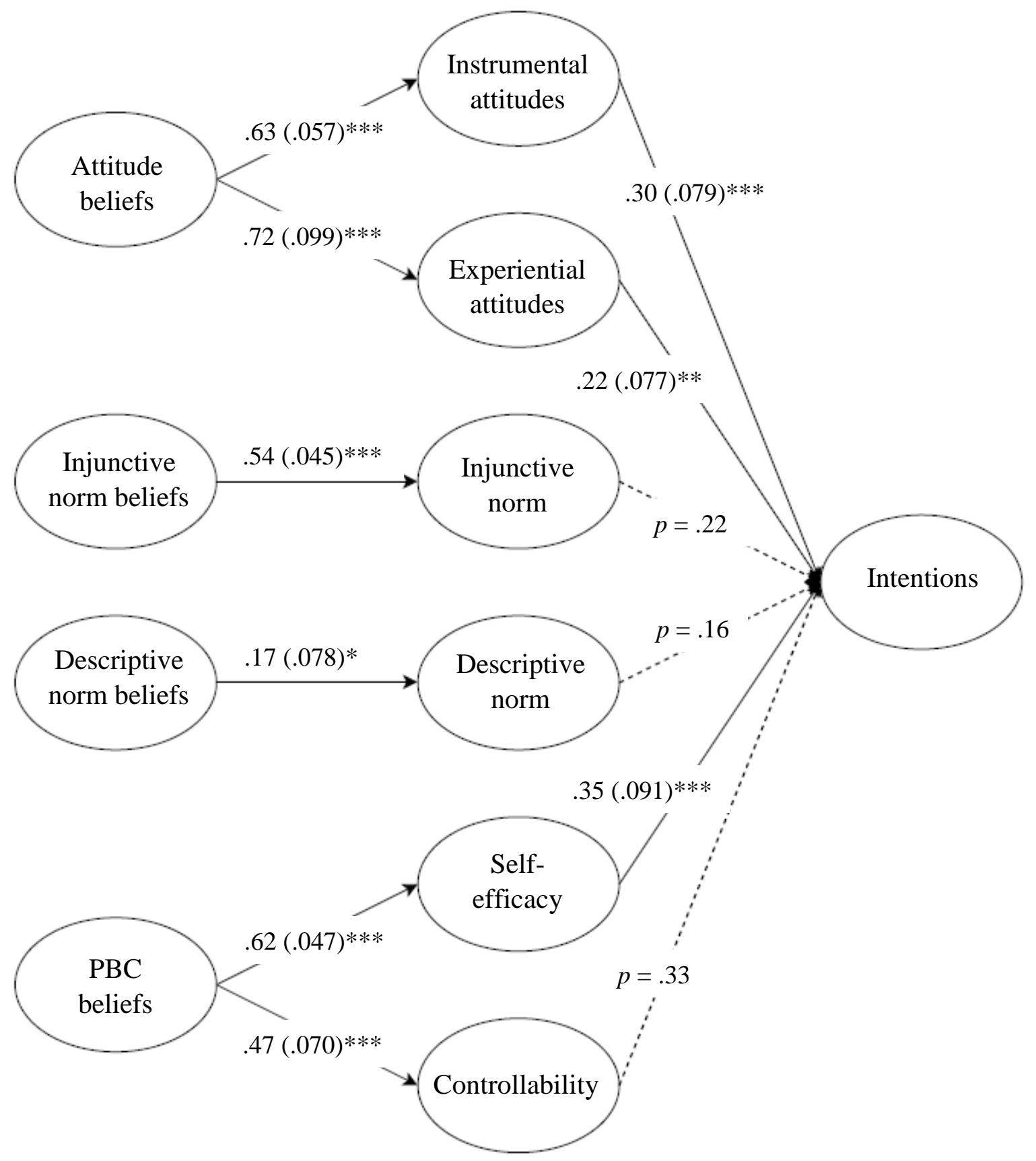

Figure 4.3. Structural equation model for the Hazard condition Dotted line indicates a nonsignificant path. For these paths, the associated numbers are the $p$ values. For significant paths, the numbers represent the standardized estimates, with the standard errors in parentheses. $R^{2}$ for intentions $=.484$. Adjusted $R^{2}$ for intentions $=.474 . * p<.05, * * p<.01, * * * p<.001$.

For both models, the belief constructs are significantly and strongly associated with their respective direct constructs; the weakest path $(\beta=.17)$ was for descriptive norm beliefs 
in the Hazard condition. These cognitions were only regressed on their relevant belief construct (i.e., attitude beliefs were only tested for associations with experiential attitudes and instrumental attitudes). The path between descriptive norm beliefs and descriptive norms was the weakest for both groups, although it was weakest in the Hazard condition as noted above. Not all direct TPB constructs contributed to the explanation of intentions and the significant paths varied between the two groups.

In the Disaster condition, experiential attitudes, injunctive norms, and self-efficacy were positively associated with intentions, while controllability was negatively associated. These findings further demonstrate the importance of the two-factor TPB, as each factor within each construct demonstrated a different association with intentions (i.e., significant and nonsignificant, or positive and negative). Overall, the model explained $42.6 \%$ of the variance in intentions, in line with typical findings using this theory (e.g., Armitage \& Conner, 2001). In contrast to the Disaster model, in the Hazard model both attitude factors were positively associated with intentions, neither of the norm factors were significantly associated, and selfefficacy was significantly associated while controllability was not. Further, the Hazard model explained $48.4 \%$ of the variance, more than did the Disaster model. These meaningful differences between the two models suggest that, while participants did not appear to differ on the constructs themselves in terms of mean scores as reported above, the way that these constructs were associated with intentions did differ. Given that the purpose of the TPB is to predict behaviour via intentions, the larger portion of the variance in intentions explained by the Hazard model is a particularly important finding. Implications of this finding, and the differences in paths, are discussed after the preliminary test for association between intentions and future behaviour is examined.

Predicting behaviour. While I did not have a comprehensive measure of subsequent behaviour to test the predictive capability of intentions, I included a proxy measure: information-seeking (Bourque, 2013). This variable was dichotomous, as participants either requested or did not request to receive an earthquake-planning guide to increase their household preparedness. Logistic regression was used to test the association between intentions and behaviour. In the Disaster condition, this test was non-significant ( $p=.181)$, suggesting that those with stronger intentions to prepare for a natural disaster were not more likely to request the earthquake-planning guide. In contrast, the odds ratio for the Hazard condition was significant, $\mathrm{OR}=.809(S E=.078), p<.05$, suggesting that those with stronger intentions to prepare for natural hazards were more likely to request the planning guide. The lack of significant chi-square cross-tabulation results comparing the ratio of those who did and did not 
request the guide between groups (presented earlier) suggests that this difference in significance of odds ratio is not due to a different pattern of responding to the behavioural item: rather that there is a difference in the association with intentions.

To clarify this difference, I conducted independent samples $t$-tests for each condition, using responses on the behavioural item as the grouping variable. In the Disaster condition, the mean intention scores did not significantly differ between those who did $(M=5.18, S D=1.29)$ and did not request the guide $(M=5.36, S D=1.28 ; t(272)=1.18, p=.240)$. In contrast, the mean intentions scores were higher in the subset of participants who did request the guide $(M$ $=5.35, S D=1.23)$ compared to those who $\operatorname{did} \operatorname{not}(M=4.92, S D=1.54 ; t(190.75)=2.42, p<$ $.05, d=0.35$ ). This finding further supports the inference that intentions are significantly associated with behaviour in the Hazard condition, but not the Disaster condition.

Exploratory analyses tested the direct effect of PBC on behaviour. Controllability and self-efficacy were included in a logistic regression predicting behaviour along with intentions, which is the method suggested by Ajzen (2011a) for testing the effect of PBC on behaviour. In the Disaster condition, neither controllability $(p=.12)$ nor self-efficacy $(p=.53)$ were significantly associated with requesting the guide. In the Hazard condition, controllability was again non-significant $(p=.89)$. The association between self-efficacy and behaviour was marginally significant, $\beta=.067(S E=.49), p=.06$, although there was no significant difference in self-efficacy between those who requested the guide $(M=5.42, S D=1.09)$ and those who $\operatorname{did} \operatorname{not}(M=5.41, S D=1.15 ; t(278)=.11, p=.91)$.

\section{Discussion}

The current study built on Study 1 by testing in a more thorough manner the applicability of the TPB to the context of household preparation for natural disasters. As part of this more thorough test, the questionnaire was extended to include both the two-level and the two-factor developments of the theory. The literature has proposed both extensions for many years (Ajzen, 2002), yet studies including either beliefs or all theorized subcomponents are rare (Conner, Hugh-Jones, \& Berg, 2011; Hardeman et al., 2002). Given that not all of the cognition components have been explored in natural hazard research, and that the belief components are argued to be a better way to effect behaviour change using the TPB than targeting the cognition components (Darker, French, Longdon, Morris, \& Eves, 2007), these inclusions seemed pertinent to developing a complete TPB model in this context.

Further, this study included a test of differences between two often-used terms in this field: natural disaster and natural hazard. Particularly given the growing argument among 
academics to stop using the term "natural disaster" (see e.g., Chmutina \& von Meding, 2019), evidence for any differences on either beliefs and cognitions or associations between these constructs will contribute meaningfully to the practical field as well as to research in the area.

\section{The Two-Level Extension to the TPB}

For both conditions, all belief measures were positively and significantly associated with their corresponding cognitions with paths ranging from .17 to .72 across both conditions. These findings support the suggestion that the TPB constructs can be predicted using indirect measures. In line with the suggestions of multiple authors (e.g., Ajzen, 2006a; Darker et al., 2007), targeting beliefs rather than cognitions may offer another avenue for effecting behaviour change. Establishing the associations between the beliefs and cognitions included in the theory is therefore an important step in identifying possible intervention strategies to ultimately increase behaviour.

\section{The Two-Factor Extension to the TPB}

This study also provides evidence for treating each cognition component as having two discrete factors, a practice which is inconsistently applied throughout the literature. The distinction between descriptive and injunctive norms has been formalized within much of the literature for over a decade, but is still seldom applied, with many recent studies continuing to use subjective norms (e.g., Sun et al., 2015; Zhu, Zhang, Ling, \& Wan 2017). The current study provides empirical support for the distinction between these two types of norms, which has been repeatedly shown outside of the TPB literature (e.g., Park \& Smith, 2007, Vinnell et al., 2018). In the natural hazard model, injunctive norms were significantly and positively associated with intentions, whereas descriptive norms were not. This finding may be due to the cognitive process involved with considering injunctive norms being more aligned with the deliberative process inherent within the TPB. The inconsistency in previous findings of a normintentions association within TPB studies could therefore be due, in part, to mixed usage of descriptive and injunctive norms, with the latter but not the former significant within the model. Some authors have also suggested that weaknesses in the norm-intention relationship is due to intentions being more dependent on personal factors (i.e., attitudes and PBC) than on social factors (i.e., norms; Topa \& Moriano, 2010). While previous research which has applied the TPB to natural hazard preparation has found significant associations between norms and intentions, those studies used subjective norms (Bates, Quick, \& Kloss, 2009; Morrison, Lawrence, \& Oehmen, 2014; Najafi et al., 2017) which are conceptually similar to injunctive norms as used here (White et al., 2009). 
Similarly, this study supports the differentiation of experiential and instrumental attitudes. In the Disaster condition, experiential attitudes were positively associated with intentions to prepare but instrumental attitudes were not, while both types of attitudes were significantly associated with intentions in the Hazard condition. Factors similar to instrumental attitudes (outcome expectancy, response efficacy, and fatalism) have repeatedly been shown to relate to preparedness (Becker et al., 2015; Lindell \& Perry, 2000; McClure, Allen, \& Walkey, 2001). The finding that instrumental attitudes are relevant for intentions to prepare for natural hazards, but not natural disasters, provides novel and valuable information about both the role of this variable in preparation and the effect that behavioural wording can have. Further, few if any studies have clearly demonstrated the importance of experiential attitudes to intentions to prepare. The significance of this factor here therefore presents another important contribution of this study. It is likely that neither of the above findings would have been demonstrated had attitudes been treated as a single construct, rather than distinguishing instrumental and experiential types.

Finally, splitting PBC into controllability and self-efficacy also provided more informative knowledge than would have testing PBC as a single construct. Previous research using the TPB in the area of natural hazards has found positive associations between PBC and intentions to prepare (Bates et al., 2009; Morrison et al., 2014; Najafi et al., 2017). However, these studies operationalized PBC as a single factor similar to self-efficacy, as is common in TPB research (Ajzen, 2011a). The findings in this study using both self-efficacy and controllability reflect those of the attitude constructs. Much previous research has demonstrated the importance of self-efficacy for preparation (Becker et al., 2015; Lindell \& Whitney, 2000), which is supported by the findings of this study. However, the role of controllability in preparation has not been thoroughly tested. The lack of association between controllability and intentions to prepare for natural hazards in comparison to the significant, negative association with intentions to prepare for natural disasters would likely not have been found without including both this subfactor and self-efficacy. This distinction, therefore, should be applied in TPB research but could still be clarified further. For example, Becker et al. (2015) suggests that within disaster research, outcome expectancy is strongly linked with self-efficacy; potential overlap in these two constructs within TPB models could be explored.

\section{Natural Disasters vs. Natural Hazards}

One aim of including the experimental manipulation of terms used in the survey was to test whether participants held more positive beliefs, cognitions, and intentions when presented with questions referring to natural hazard preparation or natural disaster preparation. Broadly, 
the results show that this framing made no difference in the extent to which people held positive beliefs, cognitions, and intentions. Further, the ratio of participants who did to those who did not request the earthquake planning guide did not significantly differ between the two conditions. For only one of the mean comparisons did the conditions differ: the direct measure of injunctive norms. Further analyses identified that this mean difference was due to a single item, which was the only scale item to refer to "people like me", rather than important others. Participants saw people similar to themselves as more approving of natural disaster preparation than natural hazard preparation but did not see this difference for important others. This finding suggests that participants perceive people who are important to them differently than people who are like them, highlighting a potential issue with the use of inconsistent measures within the TPB literature.

While the manipulation in terminology did not meaningfully impact the strength of beliefs or cognitions, this study showed that the relationships between these constructs differ depending on whether participants were asked about natural disasters or natural hazards. While overall both hazards and disaster framing models explained intentions to prepare at approximately the expected level for a TPB study (e.g., Armitage \& Conner, 2001), the natural hazard model explained more of the variance in intentions than did the natural disaster model (48.4\% versus $42.6 \%$, respectively). This difference suggests that the TPB better explains the formation of intentions to prepare at the household level for natural hazards compared to natural disasters.

Comparing the associations within the structural equation models between the two conditions provides further support for these differences. In the Disaster condition, instrumental attitudes and descriptive norms were not significantly associated with intentions, and controllability was significantly negatively associated. In comparison, in the Hazard condition instrumental attitudes were significantly associated with intentions, and controllability was not significant. As with the Disaster model, there was no significant association between descriptive norms and intentions. However, there was also no significant association between injunctive norms and intentions. Half of the paths between the constructs and intentions, therefore, differed between the two framing conditions. Further evidence for this difference is needed to establish if one of those frames is preferable over the other in terms of creating behaviour change. For example, the finding that instrumental attitudes related positively to intentions only within the natural Hazard condition could be because the term "hazard" emphasizes elements of risk which can be prevented or mitigated. 
Although the Disaster model had more statistically significant paths than the Hazard model (four versus three), the Hazard model explained more of the variance in intentions to prepare. Further, preparation intentions were not significantly associated with requesting the earthquake planning guide in the Disaster condition, but were associated in the Hazard condition, such that those with stronger intentions were more likely to request the guide. Together these findings suggest that preparation intentions and behaviour for natural hazards may be easier to predict and therefore change than preparation for a natural disaster. As the purpose of this research is to identify a number of preparation-related thoughts which can be targeted in an education campaign, it is more beneficial to propose a smaller number of possible factors which explain intentions and behaviour than a larger number of possible factors which explain less of the variance in intentions and do not demonstrate an association with behaviour. Practically, then, proceeding with the term "natural hazard" is logical.

The selection of the term "natural hazard" also aligns with current debate and understanding in the field (see e.g., Chmutina \& von Meding, 2019) and addresses the problems inherent in the term "natural disaster" as explained in Chapter 1. "Natural hazard" does not imply that damage or injury resulting from an event is attributed solely to natural processes, which is the main argument to move away from the term "natural disaster" (e.g., Blanchard, 2018). Rather, the term acknowledges that a natural process is a necessary part of the causal chain leading to a disaster but leaves room in that chain for other factors such as human behaviour. Such a view is important as attributions of damage to human actions can influence preparation-related beliefs (McClure et al., 2001; McClure, Sutton, \& Wilson, 2007). It is possible that there was no association between intentions to prepare for a natural disaster and behaviour because the term induces fatalism; disasters are events with severe negative outcomes and therefore "preparing for disaster" implies that a severely negative event is inevitable. Natural hazards, however, only have the potential to cause severe negative outcomes which potentiality leaves room for more optimism about the impacts of preparing. This suggestion is supported by the findings of this study; instrumental attitudes did not relate to intentions to prepare for natural disasters but were significantly and positively associated with intentions to prepare for natural hazards.

Further, the term "natural hazard" avoids the issue of misconception inherent in solely referring to "disasters" as the common understanding of the term "disaster" is shifting to include minor misfortunes (Furedi, 2007). Retaining the "natural" qualifier increases the likelihood that audiences will understand the term as referring to hazards such as earthquakes. Currently, little evidence supports either the argument for or against the term "natural disaster". 
This study therefore provides important preliminary evidence for the current global argument away from using the term "natural disaster".

\section{Study Strengths}

The main strength of this study is its application of the complete TPB, including both the two-level and two-factor developments. The findings here particularly support the importance of distinguishing each of the three cognition components (attitudes, norms, and PBC) into their two respective subfactors (instrumental and experiential attitudes, injunctive and descriptive norms, and self-efficacy and controllability). While not all research applying the TPB has the goal of creating behaviour change, the research which does often fails to include the belief measures. As these beliefs are argued to be the causal antecedents of attitudes, norms, and PBC (Ajzen, 2011a; Darker et al., 2007; Hardeman et al., 2002), understanding the strength of these beliefs and the extent of their influence on the congruent cognition is important for selecting intervention strategies. Similarly, several authors (e.g., Armitage \& Conner, 2001) have criticized the lack of consistency around the TPB measures used in the literature, arguing that this contributes to the conflicting findings. While refinements to the measures used here can be made, this study represents one of the most thorough tests of the theory by applying the full model, excluding only a true measure of subsequent behaviour.

The second main strength of this study is the experimental component. While much previous literature has demonstrated the importance of carefully considering wording when testing risk-related perceptions and behavioural intentions (e.g., Flusberg, Matlock, \& Thibodeau, 2017; McClure et al., 2007; McClure et al., 2001), little research has shown such a meaningful impact of a very specific wording change. This finding therefore has clear practical implications for how risks associated with natural causes are communicated.

Further, it is possible that some of the inconsistency found within the TPB literature is due to differences in wording of behaviours across studies. While the TPB has been applied to many behaviours in many domains and requires adaptation for each new behaviour, the finding here of a significant intention-behaviour association for one phrasing but not for another suggests a need for the development of a single, uniform measure for each of these behaviours which can be used consistently by different researchers. For example, a number of studies which apply the TPB to predict healthy eating behaviours have expressed that behaviour in a multitude of different ways which can lead to differences in findings such as proportion of variance in intentions explained (e.g., McEachan, Conner, Taylor, \& Lawton, 2011). This difference in wording could then lead to differences in findings within the same behaviour or class of behaviour. As far as I am aware, this possibility has not been thoroughly explored, and 
this study represents one of the first, if not the first, studies to empirically demonstrate the importance of wording of the target behaviour.

Third, this study follows the recommendations of multiple authors (e.g., Ajzen, 2006a; Ejeta, Ardalan, \& Paton, 2015) to analyse data from TPB studies using structural equation modelling, which allows for paths to be tested simultaneously. This method of analysis also allowed for more thorough testing of model fit for the individual constructs and the overall model. While in some cases this fit was only adequate, the results provide stronger evidence that the model is suitable for testing household preparation than would be found using regressions alone.

\section{Limitations}

Although the method used in this study has several strengths, there are also several limitations. As observed above, some of the individual constructs demonstrated less-than-ideal reliability and fit, suggesting that improvements can be made. This is however not unexpected, given that the measures used here have been developed and used for the first time. In particular, the attitude components need some refinement. Experiential attitudes and the indirect measure of attitudes demonstrated the lowest reliability of all tested measures. This could be due to the belief measures not capturing the most salient beliefs of the sample, or the need to differentiate the two types of attitudes at the belief level.

While not strictly a weakness, given the number of highly regarded studies in the field which employ the same design, the lack of a true behaviour measure should be addressed in order to test the predictive capability of intentions over time. Further, although the experimental manipulation provided valuable information both theoretically and practically, the fact that the individual items were worded differently between conditions limited the ability to directly compare the two models as would be preferred.

Limitations of the sample. Internet recruitment offers several benefits over other methods such as cost and time efficiency (Teo, 2013) as well as high response rates (Lonsdale, Hodge, \& Rose, 2006). However, they tend to lead to less representative samples and increase the likelihood of "bogus" respondents (Kennedy et al., 2020). Previous research in Wellington using the same recruitment method found that participants in an online survey were significantly younger than those who completed a paper-based survey using an intercept method (Vinnell, 2016), and as with this study, the sample was disproportionately female. Given the findings that age is significantly associated with both survival preparation behaviour and intentions, and previous research demonstrating differences in risk perception and related 
behaviour between genders (e.g., Finucane, Slovic, Mertz, Flynn, \& Satterfield, 2000), it is important to replicate these results using a more representative sample.

\section{Improvements for the Following Study}

Both the findings and limitations of this study provide multiple directions for future research. Recommendations for these future directions which could not be addressed in this research are presented in the General Discussion (Chapter 7). However, a number of alterations to the method were made for Study 3. Firstly, the distinctions between experiential and instrumental attitudes, as well as self-efficacy and controllability, were extended to the belief level. For example, models should allow for experiential attitudes to be explained by specific experiential attitude beliefs rather than general attitude beliefs. Given that the norms component has already been developed in this way, this step is logical and now empirically supported.

Second, as this research has the goal of suggesting thoughts and beliefs relevant to natural hazard preparation that can be targeted in a future intervention, the capability of intentions to predict behaviour over time needs to be established. Doing so requires testing intentions at one time point and then measuring behaviour at a subsequent time. Although intentions typically explain less than a third of the variance in behaviour (e.g., Armitage \& Conner, 2001), this construct remains the best predictor available within the literature.

Finally, given the aim of this research to contribute to public education campaigns, the associations found in this study need to be tested and established in a sample which is more representative of the general Wellington population. The findings of Study 1 suggest that demographic factors have a limited role in preparedness behaviour; however, replicating the findings of Study 2 in a more representative sample will lend weight to the assertion that the findings can be generalized to some other contexts, in New Zealand and internationally, with similar but not identical cultural profiles. Random sampling using publicly available lists of residents such as the electoral roll can reduce the demographic biases which tend to be inherent in the method used in this study: particularly, lower average age of the sample and considerable gender bias.

\section{Conclusion}

Overall, Study 2 achieved its main aim of obtaining empirical support for further application of the TPB within the context of natural hazard preparation. Several issues within current applications of the TPB, such as inconsistent inclusion of the two-factor and two-level distinctions, are addressed, while other issues such as the importance of consistency in the 
Chapter 4: Study 2

wording of target behaviours are identified. The next step is to test whether the intentions to prepare for natural hazards predict behaviour over time. 


\section{Chapter 5: Study 3}

\section{Introduction}

\section{Overview}

Study 3 extends the previous studies by testing the relationship between intentions to prepare and preparation behaviour over time. Based on the finding in Study 2 that intentions related to the proxy behavioural measure of information-seeking, an examination of a longitudinal relationship between these variables is warranted. Moreover, using the evidence in Study 2 suggesting that it is better to refer to natural hazards than natural disasters in the context of preparation, all participants in Study 3 will be asked about preparing for natural hazards. Beliefs, cognitions, and intentions related to natural hazard preparation will be measured at Time 1, and behaviour will be measured one month later at Time 2. Behavioural measures will include the same information-seeking item used in Study 2, as well as a Likerttype scale assessing whether participants are more prepared in general and a self-report measure of specific preparation actions carried out in the interval between Time 1 and Time 2 .

Some previous TPB intervention studies demonstrate increases in behaviour at followups within the first month but no further changes at later follow-ups (e.g., Giles et al., 2014; Joffe, Perez-Fuentes, Potts, \& Rossetto, 2016), suggesting that most impacts from such interventions occur within a month. Further, studies with longer time intervals (i.e., more than five weeks) tend to demonstrate weaker correlations between intentions and behaviour (Ajzen, 2011b). Study 3 therefore included a follow-up one month after the initial surveys to increase the likelihood of identifying effects of intentions on behaviour. Most preparation behaviours included in the list of possible actions presented to participants at Time 2 can be completed within one month. Further, unlike many other behaviours, there remains a small but unpredictable chance that a significant natural hazard event will occur in the time between the initial survey and the behavioural follow-up. While such incidences produce valuable data (Vinnell et al., 2019), such an occurrence would undermine the purpose of this study. In fact, had this study used a three month follow up as used in other studies of natural hazard preparation such as that by McClure et al. (2015), the Whakaari/White Island volcanic eruption in New Zealand, which claimed 21 lives and dominated national news headlines, would have occurred between the Time 1 and Time 2 surveys. Study 3 also uses random sampling from the electoral roll to recruit participants so as to increase the likelihood of identifying beliefs which are relevant for the majority of the population, regardless of personal experience or ethnicity. 
Doing so will strengthen the assertion that these beliefs are also likely to be important in other, similar contexts, including in New Zealand and internationally.

Finally, Study 3 explores a further and final refinement to the Theory of Planned Behaviour. Based on the evidence found in Study 2 and in previous research (reviewed in Chapter 2) that experiential attitudes are distinct from instrumental attitudes and that selfefficacy is distinct from controllability, the same distinction will be tested at the belief level of the model. That is, this study will test a model of the TPB where experiential attitudes are preceded by beliefs specific to experiential attitudes, rather than specific beliefs to attitudes generally, for example. The same test will be made for instrumental attitudes, self-efficacy, and controllability.

\section{Hypotheses}

Based on the results of Study 2, I expected that all belief measures would be significantly associated with cognitions. Further, as found in Study 2 for the "natural hazard" model, I expected that experiential attitudes, instrumental attitudes, and self-efficacy would be positively associated with intentions to prepare for natural hazards, whereas descriptive norms, injunctive norms, and controllability would not be associated. Further, I expected that intentions to prepare would positively predict preparation behaviour for all three measures conducted one month after the initial survey with a weak to moderate effect consistent with previous findings from the TPB literature (e.g., Armitage \& Conner, 2001).

Two other exploratory analyses were included. First, the proposed direct effect of PBC (self-efficacy and controllability) on behaviour was tested. Finally, I expected to find that the specified belief measures would lead to increased model fit and demonstrate stronger associations with their concordant cognitive components than the general belief measures. That is, experiential attitude beliefs would be more strongly associated with experiential attitudes than general attitude beliefs, and so on. This study, including the above hypotheses and planned exploratory analyses, was pre-registered on the Open Science Framework: https://osf.io/j2gs7/

\section{Method}

\section{Design}

Study 3 used a longitudinal design to examine whether intentions to prepare for natural hazards would predict behaviour over time. This involved testing all components of the TPB including beliefs, cognitions, and intentions at Time 1 and then testing behaviour one month later at Time 2. Three measures assessed behaviour: a single item about preparation in general over the last month, the list of items used to assess past behaviour (see Study 1 for details), and 
whether participants requested or declined an earthquake-planning guide (see Study 2 for details).

\section{Participants}

Study 3 recruited participants from the same target population as Studies 1 and 2 but used a random representative sampling method. Participants were randomly selected from a list combining the electoral rolls of the Wellington, Hutt City, Upper Hutt, and Porirua City Councils. All New Zealand citizens and residents over the age of 18 (i.e., all those eligible to vote in New Zealand elections) are required to be registered on the electoral roll. As this roll is publicly available, individuals can request to have their information removed for privacy reasons, but these exclusions should not meaningfully impact the representativeness of the electoral roll.

From this roll, 1,500 people were selected to receive an invitation to participate in the survey. This number was calculated to achieve a probability sample of approximately 383 participants at Time 1 (using the equation provided by Dillman, Smyth, \& Christian, 2014, p. 78), assuming a low response rate. A recent study of natural hazard preparedness in Wellington using a postal recruitment method achieved a response rate of approximately one-third (Doyle et al., 2018). Although a sample of this size with complete data at both time points would be preferable, assuming moderate attrition between the two time points would mean an initial recruitment size beyond the time, financial, and resource capabilities of this $\mathrm{PhD}$ project. The planned sample size of approximately 400 participants at Time 1 was expected to result in a sample size comprising data at both time points of at least 100-200 participants (e.g., Satherley et al., 2015). This is considered a minimum for structural equation modelling analyses by conventional standards (Boomsma, 1985) as well as more recent, rigorous standards (Wolf, Harrington, Clark, \& Miller, 2013).

The 1,500 selected participants were then sent a recruitment letter through the post. This letter gave a brief description of the research and provided a link for participants to access the online survey (see Appendix 5.A). The letters were posted on Tuesday the $8^{\text {th }}$ of October 2019 , with responses starting from the $11^{\text {th }}$. The final response was recorded on the $7^{\text {th }}$ of November 2019. The 87 participants who agreed to participate in the Time 2 survey were invited by email to participate in the Time 2 survey approximately one month after they started the Time 1 survey.

Time 1 sample. One hundred and fifty-three participants commenced the Time 1 survey for an approximate response rate of $10 \%$. This is much lower than the anticipated response rate of approximately one-third based on similar methods used in previous research 
with the same population (Doyle et al., 2018). Therefore, the statistical approach presented here differs from that planned in the pre-registration. The rationale for these changes is presented before the respective analyses. The information sheet and full survey for Time 1 are presented in Appendices 5.B and 5.C, respectively.

Of the 153 participants who started the survey, two participants did not answer any questions beyond the first intentions scale; therefore, their data could not be used for any analyses and were removed from the dataset. This is a much lower drop-out rate (approximately $1.3 \%$ ) than in Study 2 (36\%). All participants consented, were over the age of 18, lived in the urban Wellington region, and understood the presented definition of natural hazards so no exclusions were made for these factors. The final sample comprised 151 participants.

Demographics. Participants' ages ranged from 18 to 86 with a mean of 51.26 ( $S D=$ 16.13). This mean age is much higher than that found in the previous studies (Study $1=30.44$; Study $2=32.89$ ) and is actually further from the median age of 37.2 years established in the 2018 Census for the Wellington Region. This is likely because the Census considers all citizens, including those below the age of 18 who are not on the electoral roll. While the mean age of this sample does not align with the median age of all Wellington residents, it likely better reflects the median age of residents who are 18 years or older.

Of the 144 participants who reported their gender, 50.7\% identified as women and $49.3 \%$ identified as men. This distribution is much closer to the population of Wellington, where $51.2 \%$ are women and $48.8 \%$ men, than the samples in Study 1 (87.7\% women) and Study 2 (86.0\% women). However, the sample comprises a disproportionately large number of Pākehā (European descent: $81.9 \%$ ). Comparisons to Census data for ethnicity is not possible as the Census allowed participants to indicate more than one ethnic identities; however, Māori are particularly poorly represented here $(2.1 \%)$ compared to Study $2(7.9 \%)$. A total of $4.2 \%$ identified as Asian, 2.1\% as Pasifika, 6.9\% as European, and $0.7 \%$ (one participant) as African. No participants reported being North American or Latin American. With the exception of the proportion of participants identifying as Asian, which was 3.8\% in Study 2, all other minority ethnicities were less represented in this sample compared to that of Study 2. Implications of these increases and decreases in representativeness between recruitment methods are discussed later, including considerations for potential intervention audiences. Finally, 5.6\% had not completed secondary school, $19.4 \%$ had a secondary school qualification, $22.9 \%$ had a polytechnic or trade certificate, $25.0 \%$ had an undergraduate degree, and $29.1 \%$ had a postgraduate degree (including Masters and Doctorate). 
Time 2 sample. Sixty-three participants started the Time 2 survey for a response rate of approximately $74 \%$. Two participants did not provide their email address to link their responses to their Time 1 data so were excluded. All remaining 61 participants responded to at least one behavioural measure and consented to participate in this second survey. The mean age of this sample, 52.37 years $(S D=16.12)$, did not significantly differ from the mean age of the Time 1 sample, $p=.66$. Further, 30 identified as male and 31 as female, which again did not differ from the ratio in the Time 1 sample, $p=.84$. The ratio of participants' ethnicity identifications and education level could be not compared between the two samples as most cell sizes within the chi-square test would be too small. However, again the majority (86.9\%) identified as Pākehā and a small minority had not completed secondary school (4.9\%). The information sheet and full survey for Time 2 are presented in Appendices 5.D and 5.E, respectively.

\section{Materials}

The Study 2 survey was re-used in this study (see Appendix 5.B and 5.C). As described in the previous chapter, two scales demonstrated poor reliability. In Study 2, the experiential attitudes scale only achieved a Cronbach's alpha of $\alpha=.556$ in the Disaster condition. Because the alpha for this scale was acceptable, $\alpha=.658$, in the Hazard condition which is used in this study no refinements were made to the scale. However, the attitude belief scale demonstrated poor reliability in both the Disaster condition $(\alpha=.552)$ and the Hazard condition $(\alpha=.478)$. To address this, the Study 3 survey also included four additional questions to test whether discrete instrumental and experiential attitude beliefs demonstrate better reliability and explain their relevant cognition components (instrumental and experiential attitudes) better than a general attitude belief measure. Two questions were added to both the behavioural belief strength scale and the outcome evaluation scale. The two new behavioural belief questions read "Preparing for a natural hazard can be a fun experience" and "Preparing for a natural hazard can be a rewarding experience". Participants indicated their agreement with these statements from "Strongly disagree" (1) to "Strongly agree" (7). The two new outcome evaluation questions read "Doing something fun is:" and "Doing something rewarding is:" to which participants responded on a scale from "Extremely bad" (1) to "Extremely good" (7). One action, "Obtain a fire extinguisher for my household" was added to the list of preparation actions. The item assessing action around insurance was changed to "Make sure that my house is insured for damage caused by natural hazards" to better reflect how natural hazard insurance works in New Zealand. Cronbach's alpha was calculated using SPSS Version 25. All other analyses were run in Mplus Version 8.1 (Muthén \& Muthén, 1998-2017). 
Behaviour. Three different measures in the Time 2 survey assessed preparation (See Appendix 5.D and 5.E). The same dichotomous information-seeking measure as used in Study 2 (whether participants did or did not request the earthquake planning guide) was also included here. Further, a single question asked participants, "In the past month, how much preparation have you done for a natural hazard event?", to which they responded on a 7-point Likert-type scale from 1 (None) to 7 (A lot). Finally, the same measure of past behaviour used in Study 2, which presented a list of actions that participants could report undertaking, was used here but with the specific time frame of the past month. This list included the same 18 items as used previously but also included one extra item, "Obtain a fire extinguisher for my household". These three measures provide a single-item dichotomous outcome, a single-item continuous outcome, and a multi-item continuous outcome.

Reliability. The natural hazard experience scale demonstrated a Cronbach's $\alpha$ of .55, lower than the Study 2 finding of .64 but similar to that of Study 1 (.57). Corrected item-total correlations ranged from .23 to .36 , and the reliability of the scale could not be increased by removing any of the items, indicating that no single item contributed to the low alpha score.

All direct TPB scales demonstrated adequate to good reliability: intentions (.87), instrumental attitudes (.95), experiential attitudes (.69), perceived injunctive norms (.83), descriptive norms (.89), self-efficacy (.79), and controllability (.67). Injunctive norm beliefs (.87) and descriptive norm beliefs (.86) also both demonstrated good reliability, as did PBC beliefs both when including all four items (.82) and when splitting into controllability beliefs (.70) and self-efficacy beliefs (.89). These latter two are Spearman-Brown coefficients which are a more appropriate test of reliability for two-item scales than Cronbach's $\alpha$ (Eisinga, Grotenhuis, \& Pelzer, 2013). However, the general attitude belief measure (.55) as well as both instrumental attitude beliefs (.30) and experiential attitude beliefs (.20) demonstrated poor reliability based on the threshold stipulated in the pre-registration. The analyses showed that removing either item 5 (expensive) or item 6 (complicated) increased the reliability of the general attitude belief measure to .73 or .64 , respectively. Further, the reliability of the instrumental attitude belief measure was increased to .69 with the "expensive" item deleted, while the experiential attitudes belief measure was increased to .76 with the "complicated" item was deleted. Therefore, the following analyses do not include either the "expensive" or "complicated" attitude belief items.

Individual confirmatory factor analyses identified how well the data fit the imposed structure for each TPB construct (Table 5.1). Only four constructs required a single modification to achieve adequate fit. 
Table 5.1

Confirmatory factor analysis fit criteria results for individual TPB constructs.

\begin{tabular}{lllllllll}
\hline Cognition & Factor & $\chi^{2}$ & df & $p$ value & Ratio & CFI & RMSEA & SRMR \\
\hline Attitudes & Instrumental & 3.37 & 2 & .186 & 1.69 & .986 & .068 & .013 \\
& Experiential & 5.36 & 2 & .069 & 2.68 & .963 & .106 & .042 \\
& Beliefs & 10.32 & 2 & .006 & 5.16 & .949 & .166 & .034 \\
& Item 4 with 3 & 0.02 & 1 & .894 & 0.00 & 1.00 & .000 & .001 \\
\multirow{3}{*}{ Norms } & Injunctive & 5.12 & 2 & .075 & 2.56 & .969 & .104 & .031 \\
& Descriptive & 0.49 & 2 & .781 & 0.25 & 1.00 & .000 & .007 \\
& Injunctive beliefs & 12.55 & 2 & .002 & 6.28 & .932 & .189 & .042 \\
& Item 2 with 1 & 0.02 & 1 & .886 & 0.00 & 1.00 & .000 & .001 \\
& Descriptive beliefs & 5.80 & 2 & .055 & 2.90 & .964 & .114 & .045 \\
PBC & Self-efficacy & 9.05 & 3 & .029 & 4.53 & .934 & .117 & .074 \\
& Controllability & 27.86 & 3 & .000 & 9.29 & .737 & .238 & .160 \\
& Item 3 with 2 & 5.79 & 2 & .059 & 2.90 & .961 & .112 & .070 \\
& PBC beliefs & 19.02 & 2 & .000 & 9.51 & .852 & .242 & .067 \\
& Item 3 with 1 & 3.04 & 1 & .081 & 3.04 & .982 & .119 & .015 \\
& & 3.96 & 2 & .138 & 1.98 & .983 & .081 & .026 \\
\hline
\end{tabular}




\section{Results}

\section{Descriptive Statistics}

Table 5.2 presents the means for each of the TPB constructs at Time 1. Means and standard deviations are presented for the general attitude and PBC belief scales (i.e., without the two-factor split) as well as for specific experiential and instrumental attitude beliefs and specific self-efficacy and controllability beliefs.

Table 5.2

Descriptive statistics for all TPB scales.

\begin{tabular}{|c|c|c|c|c|c|}
\hline Cognition & Factor & $N$ & Mean & $S D$ & Range \\
\hline \multirow[t]{6}{*}{ Attitude } & Experiential & 148 & 4.23 & 1.11 & 1 to 7 \\
\hline & Instrumental & 148 & 6.44 & 1.01 & 1.25 to 7 \\
\hline & Beliefs & & & & \\
\hline & General & 148 & 26.33 & 5.26 & 14 to 42 \\
\hline & Experiential & 148 & 23.46 & 6.73 & 8.67 to 39.67 \\
\hline & Instrumental & 148 & 29.31 & 6.67 & 10 to 49 \\
\hline \multirow[t]{5}{*}{ Norms } & Injunctive & 148 & 5.44 & 1.09 & 1.50 to 7 \\
\hline & Descriptive & 148 & 3.95 & 1.36 & 1 to 7 \\
\hline & Beliefs & & & & \\
\hline & Injunctive & 147 & 18.58 & 10.40 & 1.50 to 49 \\
\hline & Descriptive & 146 & 14.49 & 6.26 & 4 to 33.25 \\
\hline \multirow[t]{6}{*}{ PBC } & Self-efficacy & 146 & 5.67 & .99 & 3 to 7 \\
\hline & Controllability & 146 & 6.05 & .88 & 3.50 to 7 \\
\hline & Beliefs & & & & \\
\hline & General & 145 & 14.75 & 6.55 & 1 to 32.20 \\
\hline & Self-efficacy & 145 & 10.96 & 5.09 & 1 to 21 \\
\hline & Controllability & 145 & 20.43 & 10.98 & 1 to 49 \\
\hline Intentions & & 139 & 5.26 & 1.44 & 1 to 7 \\
\hline
\end{tabular}

Note. All direct measures had a possible range of 1 to 7 , with a midpoint of 4 . All belief measures had a possible range of 1 to 49 , with a midpoint of 25 . within the model. 
Correlations. Correlations between intentions and each of the cognition components were calculated in Mplus to control for the error in the observed items (see Table 5.3). As expected, intentions correlated positively with all cognition components, with correlations ranging from .25 to .40 . Most components were correlated with each other, with the exception of descriptive norms which only correlated significantly with instrumental attitudes and injunctive norms.

Table 5.3

Correlations between intentions and TPB cognition components.

\begin{tabular}{|c|c|c|c|c|c|c|c|}
\hline & Intentions & Inst Att & Exp Att & Inj Norm & Des Norm & SE & Control \\
\hline Intentions & - & $.39 * * *$ & $.40 * * *$ & $.26 *$ & $.29 * *$ & $.38 * * *$ & $.25 *$ \\
\hline Inst attitudes & & - & $.52 * * *$ & $.36 * * *$ & $.17 *$ & $.28 * *$ & $.35 * * *$ \\
\hline Exp attitudes & & & - & $.28 * *$ & $.12 n s$ & $.38 * * *$ & $.39 * * *$ \\
\hline Injunctive norm & & & & - & $.42 * * *$ & $.33 * *$ & $.28 *$ \\
\hline Descriptive norm & & & & & - & $.16 n s$ & $-.01 n s$ \\
\hline Self-efficacy & & & & & & - & $1.02 * * *$ \\
\hline Controllability & & & & & & & - \\
\hline
\end{tabular}

Note. Inst $=$ Instrumental, Exp = Experiential, Inj = Injunctive, Des = Descriptive, SE = Selfefficacy, Control $=$ Controllability. $*=p<.05, * *=p<.01, * * *=p<.001, n s=p>$ or $=.05$

\section{Model Fit}

The lower-than-expected sample size did not provide enough power to test a full latent structural equation model which includes all TPB constructs as well as behaviour; therefore, simpler structural equation models explaining intentions to prepare without behaviour variables were tested. In these models, each of the scale items were treated as observed variables loading on latent constructs. Intentions to prepare and $\mathrm{PBC}$ were then tested as predictors of preparation behaviour in separate analyses. To explore the two-factor distinction at the belief level, four different models were tested for fit: one with the general attitude and PBC beliefs as used in Study 2, one with only attitude beliefs differentiated, one with only PBC beliefs differentiated, and one with both beliefs differentiated. Table 5.4 presents the results of confirmatory factor analyses testing the fit of an imposed TPB structure with all observed items used to explain 
their relevant construct (excluding the two attitude belief items described in the above Reliability section).

Table 5.4

Fit criteria results for TPB models including and excluding two-factor belief distinctions.

\begin{tabular}{lccccccc}
\hline Model & \multirow{2}{*}{$\chi^{2}$} & df & $p$ value & Ratio & CFI & RMSEA & SRMR \\
\hline Beliefs not split & 1376.08 & 845 & .000 & 1.63 & .851 & .065 & .077 \\
Attitude beliefs split & 1488.29 & 837 & .000 & 1.78 & .829 & .070 & .101 \\
PBC beliefs split & 1518.90 & 837 & .000 & 1.82 & .809 & .073 & .099 \\
Both beliefs split & 1579.12 & 828 & .000 & 1.91 & .789 & .078 & .120 \\
\hline
\end{tabular}

Model fit decreased as more differentiations were added. It is possible that this loss in fit is due to a decrease in power as more constructs were added to the model. The model with both attitude and PBC beliefs split demonstrated acceptable fit on the chi-squared statistic and reasonable fit on the RMSEA statistic (Vandenberg \& Lance, 2000). The CFI statistic is lower than the typical threshold of .90 ; however, this measure penalizes for sample size so lower values are expected for this data and not indicative of poor fit. Therefore, both the model with beliefs not split, as per Study 2, and the model with both beliefs split will be estimated using structural equation modelling to test if differentiating the specific attitude and PBC beliefs changes the paths 


\section{Structural Equation Modelling}

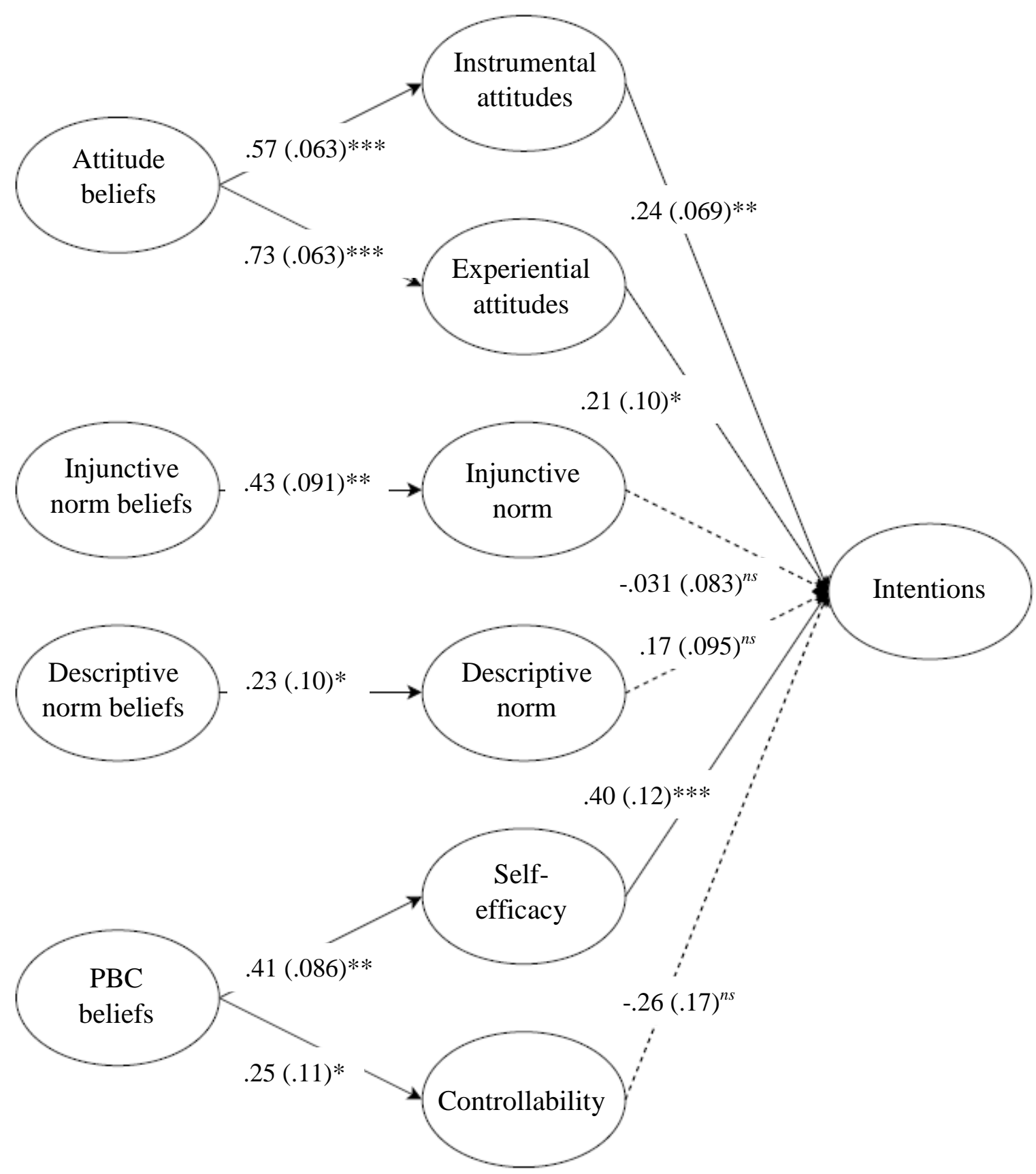

Figure 5.1. Structural equation model with attitude and PBC belief components not split. Adjusted $R^{2}$ for intentions $=.36 . p<.01{ }^{*}=p<.05, * *=p<.01, * * *=p<.001,{ }^{\mathrm{ns}}=p>$ or $=$ .05 . 
Figure 5.1 presents the structural equation model with attitude and PBC beliefs not split. This model explained approximately $36 \%$ of the variance in intentions to prepare (based on the adjusted $R^{2}$ value), close to the average percentage of variance in intentions found in metaanalyses and literature reviews (e.g., Armitage \& Conner, 2001). As expected, all belief constructs significantly associated with their related cognitions. Further, instrumental attitudes, experiential attitudes, and self-efficacy were significantly associated with intentions to prepare, consistent with the hypothesis in the pre-registration and findings for the "Hazard" condition model in Study 2. Wald tests comparing the strength of each of the significant paths between instrumental attitudes, experiential attitudes, and self-efficacy and intentions demonstrated no significant differences ( $p$ values ranged from .14 to .58).

Figure 5.2 presents the results of structural equation modelling with all belief factors differentiated. As with the previous model, all belief constructs were significantly associated with their related cognitions. Neither experiential attitude beliefs nor instrumental attitude beliefs demonstrated a noticeably different association than did the general attitude beliefs construct in the previous model. However, both the controllability beliefs and self-efficacy beliefs construct demonstrated stronger associations with their related cognitions than did the general PBC belief construct in the previous model. Implications of these findings and suggestions for future research are presented in the following Discussion section. 

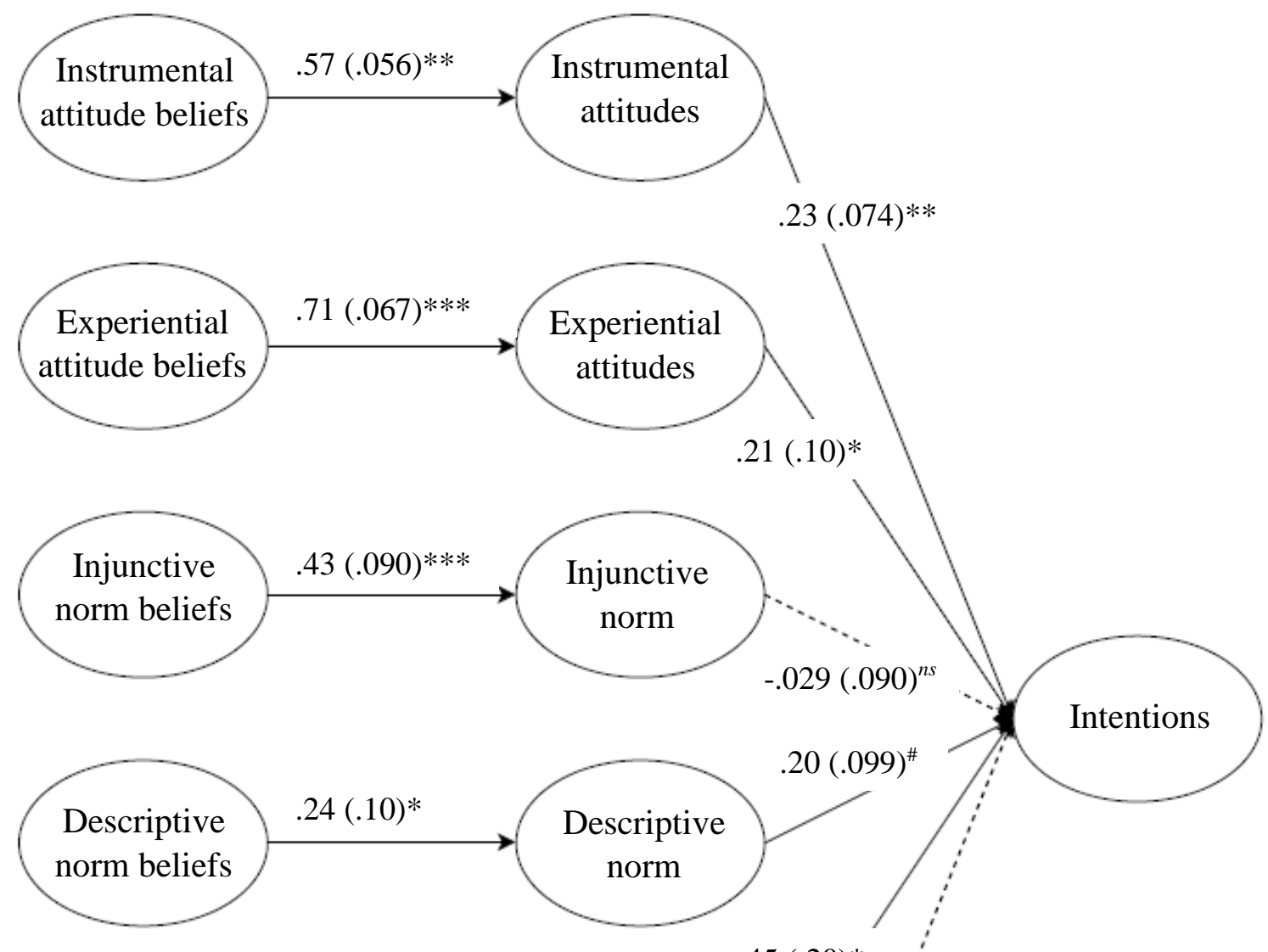

$.45(.20)^{*}$

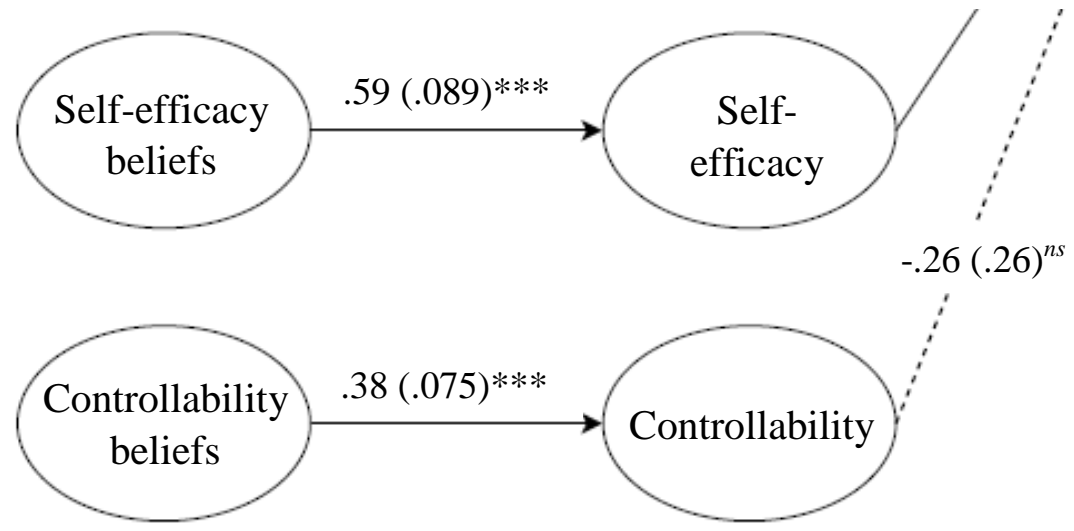

Figure 5.2. Structural equation model with attitude and PBC belief components split. Adjusted $R^{2}$ for intentions $=.28 . p<.01 . *=p<.05,{ }^{*} *=p<.01, * * *=p<.001,{ }^{n}{ }^{n}=p>.05,{ }^{*}=p=$ .05 . 
In the model with the belief constructs differentiated, instrumental attitudes, experiential attitudes, and self-efficacy were also significantly associated with intentions to prepare, with similar strength paths to the previous model (see Figure 5.2). In addition, descriptive norms were also significantly associated with intentions to prepare in the model where the belief measures are differentiated, while the amount of variance in intentions explained was lower: approximately $28 \%$ based on the adjusted $R^{2}$ value. Differences between the two models and implications for the structuring of belief components are discussed below.

\section{Predicting Behaviour}

To recap, three different behaviour measures were used; the same dichotomous information-seeking measure as used in Study 2 (whether participants did or did not request the earthquake planning guide), a single-item continuous measure using a 7-point Likert-type scale asking participants how much preparation they have made over the past month, and participants' self-reports of specific preparation behaviours between Time 1 and Time 2, with the total number of actions representing the variable value.

Requesting the earthquake-planning guide. Forty-three participants requested the earthquake planning guide and 18 declined. Two logistic regression analyses tested whether the Time 1 data predicted if participants requested an earthquake planning guide at Time 2. The first test included only intentions, while the second included the two PBC factors, selfefficacy and controllability, to examine the inconsistent direct effect of PBC on behaviour with intention controlled for (for similar methods, see Chow \& Chen, 2009 or de Vries, Dijkstra, \& Kuhlman, 1988). This method is more common (Ajzen, 2011a) and recommended by Ajzen (2002), rather than testing for an interaction of PBC with intentions as PBC is proposed to have a unique effect on behaviour. In contrast with Study 2, intentions to prepare alone did not significantly predict requests for the earthquake guide, $p=.83$. When self-efficacy and controllability were included in the model, intentions again did not significantly predict behaviour, $p=.90$, nor did controllability, $p=.96$. However, self-efficacy was a significant predictor, $\mathrm{OR}=.002,(S E=.046), p<.001$, such that the odds of requesting the guide decreased when participants had higher self-efficacy, consistent with Study 2. This finding supports the inference in the previous study that those who see themselves as more capable of preparing for natural hazards see less need for additional information or preparing tools such as a planning guide.

Self-reported general preparation. Two linear regressions tested whether Time 1 data predicted how much preparation participants reported undertaking at Time 2 since Time 1, on a 7-point scale from "None" to "A lot" $(M=2.03, S D=1.54)$. As above, the first test included 
only intentions while the second test also included self-efficacy and controllability. In the first regression, intentions positively and significantly predicted behaviour, $\beta=.25(S E=.10), p<$ .05 , demonstrating that those participants who had stronger intentions to prepare at Time 1 reported having done more preparation at Time 2 than those who had weaker intentions. However, when self-efficacy and controllability were included in the regression along with intentions, no factors significantly predicted behaviour ( $p=.90, .93$, and .49 , respectively).

Given the consistent lack of association between controllability and either intentions or behaviour in this and previous studies (with only one exception in Study 2, where controllability was negatively associated with intentions to prepare for natural disasters), a third regression tested only intentions and self-efficacy as predictors of behaviour ${ }^{7}$. In this regression, self-efficacy again was not significantly associated with preparation, $p=.22$. However, intentions significantly predicted preparation, $\beta=.22(S E=.096), p<.05$. This exploratory analysis suggests that conceptual overlap of intentions with the controllability variable suppressed the association between intentions and behaviour.

Self-reported specific preparation. As above, two linear regressions tested whether Time 1 data predicted the number of specific preparation actions reported by participants at Time 2, which ranged from zero to 15 out of a possible 19 actions $(M=2.76, S D=3.21)$. As with the previous regression on the general preparation measure, intentions to prepare alone significantly and positively predicted the number of preparation actions undertaken, $\beta=.20$ $(S E=.088), p<.05$. However, and again similar to the above analysis, when self-efficacy and controllability were included in the regression along with intentions, no factors significantly predicted behaviour, $p=.96, .96$, and .92 , respectively. The same rationale as presented above justified a third linear regression including only intentions and self-efficacy as predictors of behaviour. As for the single-item measure of behaviour, self-efficacy was again nonsignificant, $p=.96$, but intentions significantly predicted behaviour, $\beta=.20(S E=.090), p<$ .05 .

\section{Identifying Beliefs}

As the reason for including beliefs in the TPB is typically to identify precise potential targets for interventions (Ajzen, 2006a), final regressions examined the direct association between belief scale items and their related cognition for the three significant explanatory components (instrumental attitudes, experiential attitudes, and self-efficacy) to identify the

\footnotetext{
${ }^{7}$ This test was not run for the behaviour of requesting an earthquake-planning guide as intentions alone did not predict behaviour and therefore controllability could not have a suppressive effect.
} 
most important beliefs within each construct to target. Both instrumental attitude beliefs were significantly associated with instrumental attitudes: the belief that preparing will result in getting through a natural hazard event better, $\beta=.38(S E=.094), p<.001$, and that preparing is a life-saving action, $\beta=.25$ ( $S E=.068), p<.001$. A follow-up Wald test comparing the strength of these paths was non-significant, $p=.22$, suggesting that the two beliefs are relatively equally important predictors of instrumental attitudes.

Similarly, both experiential attitude beliefs were significantly associated with experiential attitudes: preparing can be a fun experience, $\beta=.43(S E=.095), p<.001$, and preparing can be a rewarding experience, $\beta=.31,(S E=.10), p<.01$. Again, a follow-up Wald test comparing the strength of the paths was non-significant, $p=.41$, suggesting that both beliefs are relatively equally important. Only participants' belief that they will be able to put in the effort to prepare was significantly associated with self-efficacy, $\beta=.34$ ( $S E=.13), p<$ .05 . Participants' belief that they will think about preparing was not significantly associated with self-efficacy, $p=.88$.

\section{Discussion}

\section{Chapter Summary}

This study extended Study 2 in two main ways. First, participant recruitment used representative sampling with postal addresses randomly selected from Wellington electoral rolls. This method aimed to achieve a more representative sample than that used in Studies 1 and 2, which demonstrated a strong gender bias in particular. Although this demographic factor did not significantly relate to intentions to prepare in either Study 1 or Study 2, a more representative sample is desirable given that this research aims to inform broad, non-targeted public education campaigns. While the method used in Study 3 did result in a balanced gender ratio similar to the actual ratio in Wellington's population, the mean age of participants was considerably higher than the median population age according to Census data for Wellington (although this is likely due to the Census including those under the age of 18). Further, minority groups, in particular Māori, are less represented in this data in comparison to Study 1 and 2.

The second extension was the inclusion of a follow-up survey containing both objective and self-reported behavioural measures, completed by a subsection of participants who completed the first survey. This longitudinal method allowed for the testing of intentions to prepare as a predictor of subsequent preparation behaviour and therefore supports that increasing intentions to prepare will have the intended effect of increasing preparation behaviour. Overall, the results demonstrate the expected association between intentions to prepare and behaviour, when behaviour was measured with a continuous rather than a 
dichotomous variable (Ajzen, 2011a), although intentions did not significantly predict whether participants requested an earthquake planning guide (the objective measure) as was found in Study 2. This effect may not have been found in this study due to either the longer time frame between the two measures or the smaller sample size.

\section{Discussion of Results}

Intentions. As predicted, instrumental attitudes, experiential attitudes, and self-efficacy were significantly and positively associated with intentions to prepare for natural hazards in both the model without the belief factor divided (as used in Study 2) and in the model with these factors differentiated, explaining approximately $36 \%$ and $28 \%$ of the variance, respectively. Although the latter model explained less of the variance in intentions, a fourth factor, descriptive norms, also demonstrated a significant association with intentions.

The amount of variance explained in these two models is within the range typical for TPB studies (e.g., Armitage \& Conner, 2001), though is towards the low end of expected effect sizes. While adjusted $R^{2}$ values are usually lower than non-adjusted values, it is likely that the lower variance explained here than in most previous research is a result of using structural equation modelling which controls for errors associated with all items, which is not the case in the typical regression approaches used in many TPB studies. Overall, however, this study replicates the findings of Study 2 that instrumental attitudes, experiential attitudes, and selfefficacy meaningfully relate to intentions to prepare for natural hazards and implies that these factors have relatively equal impacts; future research should confirm this inference with a larger data set.

Beliefs. This study tested a further and final refinement to the TPB model by introducing the two-factor attitudes and PBC distinction at the belief level. Across both the model where these belief factors were not split and the model including the distinction, all belief components were positively and significantly associated with their relevant cognition. Differentiating instrumental and experiential attitude beliefs did not result in stronger associations with the related direct attitude factors than did modelling general attitude beliefs. However, both self-efficacy beliefs and control beliefs demonstrated stronger associations with the related control factors than did general PBC beliefs. Meaningful differences between the two models, including the number of significant paths and the $R^{2}$ values, suggests that further, more rigorous testing of this theoretical distinction would be useful. Using this distinction will help to identify which specific beliefs contribute to the development of intentions to prepare. In relation to the primary aim of suggesting factors for a behaviour change campaign to target, five beliefs were found to be important: the belief that preparing will (1) get the individual 
through a hazard event better, (2) is a life-saving action, (3) can be fun, (4) can be rewarding, and (5) is an effort of which they are capable. Applications for these important, novel findings will be presented in detail in Chapter 7 (General Discussion).

Behaviour. The primary contribution of this study to the thesis is to establish that intentions to prepare for natural hazards statistically predict subsequent behaviour. In contrast with the cross-sectional results of Study 2, intentions at Time 1 did not predict whether participants requested an earthquake planning guide at Time 2. Consistent with Study 2, however, self-efficacy did predict this behaviour in Study 3, with those scoring higher being less likely to request the guide. This finding is unsurprising in that those who see themselves as more capable of preparing for natural hazards would logically be less likely to gather information on how to undertake this behaviour. The lack of association between information seeking and intentions to prepare suggests that the behaviour of information-seeking is perceived differently than the behaviour of actually undertaking preparation actions (Paton, Smith, \& Johnston, 2005; Paton, Johnston, Smith, \& Millar, 2001).

Another explanation for the lack of association between intentions and informationseeking is that the intention-behaviour association is less likely to be significant when the measures used are not aligned as is the case here (Ajzen, 2011a). When behaviour was measured as continuous, intentions to prepare positively and significantly predicted preparation behaviour, with associations that were weak to moderate in strength. Intentions, therefore, are important to encourage in terms of increasing preparation for natural hazards. However, the well-documented intention-behaviour gap (Sniehotta, Scholz, \& Schwarzer, 2005) suggests that a second method for increasing behaviour specifically targeted at this problem may be beneficial, as across all studies intentions to prepare are at a moderate level.

Several suggestions have been made within the literature to increase the translation of intentions into behaviour, including goal setting and implementation intentions (Ajzen, 2006a; Ajzen, 2001a; Hardeman et al., 2002; Gollwitzer, 1999; Riebl et al., 2015; Webb \& Sheeran, 2006). These aligned concepts can be applied together (Hardeman et al., 2002) where participants break down a particular broad task such as preparing for natural hazards into smaller tasks (goal setting; Locke, Shaw, Saari, \& Latham, 1981) and decide how, when, and (if relevant) where they will undertake those smaller tasks (implementation intentions; Gollwitzer, 1999). Implementation intentions are commonly used in terms of associating a particular behaviour with an environmental cue; for example, the intention to implement "drop, cover, and hold" when an earthquake occurs. Associating preparation behaviour with an 
earthquake, however, is likely less beneficial as once the cue (i.e., the earthquake) has occurred, the behaviour will not be effective (i.e., reducing negative impacts of the earthquake).

Goal setting has been used to increase preparation for earthquakes by Wellington businesses (McClure, Spittal, Fischer, \& Charleson, 2015) and implementation intentions have been used successfully to supplement the TPB in behaviour change interventions as a way of bridging the intention-behaviour gap. For example, implementation intentions have fully mediated the effect of intentions on physical activity in the study by Sniehotta and colleagues (2005) and increased the effectiveness of an intervention to reduce snacking behaviour (Karimi-Shahanjarini, Rashidian, Omidvar, \& Majdzadeh, 2013).

As well as increasing behaviour, interventions using action planning (similar to implementation intentions) can also increase self-efficacy (Williams \& French, 2011). This concept of action planning has been found to interact with coping planning (Arau'jo-Soares, McIntyre, \& Sniehotta, 2009). Coping planning entails imagining potential future barriers to undertaking a behaviour and, importantly, deciding on ways to deal with those barriers (Sniehotta, 2009), which aligns closely with self-efficacy (Luszczynska, Schwarzer, Lippke, \& Mazurkiewicz, 2011). Luszczynska et al. (2011) found that planning was more effective for participants with higher self-efficacy, suggesting that while an intervention targeting selfefficacy should have a direct effect on behaviour, an intervention which also includes implementation intentions and coping planning would be more effective.

\section{Implications}

Theory of Planned Behaviour. This study supports the suggestions of previous studies that the two-factor distinction in the cognition components of the TPB should be applied to all components: attitudes, norms, and PBC. Further, the differences between the models where this two-factor distinction is and is not included at the belief level supports the view that this distinction should be made, though perhaps with a more rigorous method than was implemented in this research. Rather than being added as an amendment once the belief measures have been developed, this distinction should be made when the belief measures are developed. Although this research provides a set of measures which can be easily adapted to different behaviours, different salient attitude and control beliefs for each new behaviour should be ascertained at the outset and used to adapt the items employed here.

In addition, comparisons of the different behaviour regressions suggest that there may be some overlap in the items for intentions and controllability, although the two variables are only weakly correlated. Ajzen (2011a) argued that the distinction of the PBC variable into controllability and self-efficacy may only be important for some behaviours. It is possible that 
in the context of natural hazard preparation, rather than not applying this distinction, only selfefficacy needs to be included in the model as controllability does not appear relevant. As well as accounting for differences between specific behaviours within a single category (e.g., information seeking as a preparation behaviour) it may be important to consider the different behavioural context. People typically perceive household preparation as their responsibility (Arlikatti, Lindell, \& Prater, 2007) which may limit the impact of controllability.

Natural hazard preparation campaigns. While the concepts of outcome expectancy and fatalism (similar to instrumental attitudes; see Study 4) are well supported as relevant to natural hazard preparation (Johnston et al., 2013; Lindell \& Perry, 2000), this study offers specific aspects of these concepts that could be targeted. For example, it is useful to know that communicating preparing as beneficial, without having to specify particular benefits, is likely to be effective, and that specifically communicating that these actions can be life-saving is likely to increase behaviour. Given that survival actions are undertaken at a higher rate than mitigation actions (see Study 1), this latter finding suggests that emphasizing how steps such as strengthening the foundations of a house saves the lives of its occupants (as well as reducing damage) could be an effective way to encourage such behaviour.

In contrast with instrumental attitudes, previous research in the natural hazard field has not tested experiential attitudes as a predictor of preparation. While several programmes locally and internationally have aimed to make both understanding risk and preparation actions engaging for children using games (e.g., Dreaver, 2018), little research has tested the same for adults. Finally, despite the finding in Study 1 that "hadn't thought about it" was the most common reason for not undertaking 11 out of the presented 18 actions, participants' belief that they will think about preparing was not significantly associated with self-efficacy, whereas the belief that they will be able to put in the necessary effort was. It is possible that participants think about preparing generally, rather than specifically as was assessed in Study 1 and McClure et al. (2015), and that it is lack of willingness to put in the effort that inhibits them acting rather than not thinking about it.

\section{Limitations}

Sample size. Contrary to expectations based on previous similar research, only a relatively small sample was obtained for this study. This difference is possibly due to the new method used here of providing an online link for participation rather than a paper survey and a return envelope, although no rural areas were sampled, and internet access is nearly universal across New Zealand (InternetNZ, 2017). 
Due to this smaller than expected sample, the data collected was underpowered to test behaviour as part of the full model. Testing the intention-behaviour association separately did not allow for a test of the proportion of variance explained in behaviour as planned in the preregistration. This low response rate also increases the likelihood of a self-selection bias, as possibly only participants who had either strongly positive or strongly negative views of preparing were motivated to take part in the study. This bias would likely extend to the subset of participants who also completed the Time 2 survey. Such a bias also ought to be considered when planning any research using the same recruitment method.

Demographics. This study used postal recruitment because the electoral roll is the best way to select a random sample from the whole population, and the only contact information available on this roll is street addresses. The rationale for this method was to obtain a more representative sample as those in the previous studies demonstrated sampling biases, particularly regarding gender. The sample in Study 3 had a gender ratio accurately reflecting the Wellington population, but the average age of participants was further than Study 1 and Study 2 from the median age for the Wellington Region (although this median age is based on the total population, including those under the age of 18). Further, minority ethnicity groups, with the exception of participants who identified as Asian, were less represented than in Study 2. Some studies might deliberately aim for better representation of gender or ethnicity distribution, in which case the difference in demographics between the two recruitment processes would suggest how to recruit participants. However, the overall purpose of this research is to inform a non-targeted intervention to be run in the future and the findings show only weak effects for a small number of demographic variables. This suggests that for the purposes of this research, online recruitment such as that used in Studies 1 and 2 produced a higher quantity of equally, if not more, representative data for less time and financial investment than did the postal method used in Study 3.

While Study 1 suggested that ethnicity is not associated with preparation behaviour, given Wellington's multicultural population it is important to identify beliefs which are relevant to preparation across different ethnic groups, so that any intervention based on those beliefs is likely to be at least partially effective regardless of the cultural makeup of the audience, particularly in New Zealand. Similarly, direct experiences of natural hazard impacts are likely to vary somewhat within the Wellington Region; for example, those who live in the CBD and were impacted by cordons and witnesses building damage following the 2016 Kaikōura earthquake potentially recall this event differently than those living in the lower Hutt Valley. Those residents experienced strong shaking but little damage in the earthquake, and 
the next day experienced extensive flooding due to heavy rain, similar to that which led to one fatality in the area the year before. The consistency between the findings of Study 2 and Study 3 with their different recruitment methods strengthens the assertion that the factors identified are likely to be broadly relevant within populations comprising people of different ethnicities and levels and types of experience.

\section{Strengths}

Study 3 has several strengths in statistical, theoretical, and practical respects. First, the replication of Study 2 findings in this sample with a more balanced gender ratio suggests that impacts of this demographic bias are negligible. Second, and as noted in the Study 2 discussion, the use of structural equation modelling to test the how the TPB cognitions explain the variance in intentions allows for more confidence in the findings than if regression models had been used (Ajzen, 2011a; Topa \& Moriano, 2010), although this test would ideally have included behaviour had the data been sufficiently powered.

Third, inconsistencies in how behaviour is measured, both within and across TPB studies, likely contributes to conflicting findings. For example, the association between intentions and behaviour has been found to be both weaker (Webb \& Sheeran, 2006) and stronger (Armitage \& Conner, 2001) when the behaviour measure is self-report. Though Ajzen (2011a) argues that the intentions and behaviour measures should be compatible within studies, this recommendation is not applied in all TPB studies. The inclusion of three different behaviour measures, then, presents a significant strength of this study. The lack of association between intentions and requesting the planning guide is consistent with the claims of Ajzen (2011a) as intentions are general and continuous while the behaviour is specific and dichotomous, although this association was significant in Study 2. Given the much smaller sample in this study compared to Study 2 and the longer delay between when participants reported intentions and the time that they could request the guide (Ajzen, 2011b), it is possible that there may be an association, but it is not strong enough to be detected after a month or with a small sample.

Although the multi-item behaviour measure is self-report, it assesses objective actions rather than a holistic judgment as is required in the single-item behaviour measure. Consistent with findings from Armitage and Conner's (2001) meta-analysis that self-report measures demonstrate stronger associations than objective measures, intentions predicted the single-item behaviour measure better than the multi-item behaviour measure. It is possible, however, that this small difference in paths is due to the single-item behaviour measure being more similar to the intentions measure (i.e., response on a 7-point scale, referring to the behaviour generally). 
While these two potential explanations cannot be tested here, the differences in associations found in this study support the careful evaluation and future exploration of the role of behaviour measure construction.

Finally, this study tests the full TPB model, including behaviour, all proposed subcomponents, the level of antecedent beliefs, and the two-factor distinction at that belief level. In addition, although the small dataset did not provide sufficient power to test this full model with all variables included at once, this study demonstrates that it is feasible to present all necessary items in a single study. Although only a small proportion of those invited started the survey, the vast majority of those who started completed it. Using the full model in this way allows for the identification of which attitudes, norms, and control perceptions are relevant to intentions and behaviour, and goes beyond testing broad concepts such as outcome expectancy and selfefficacy to include specific relevant beliefs which can be more easily and directly targeted in public education campaigns. Previous research using the TPB suggests that behaviour change interventions to target specific beliefs are more effective than interventions to target the cognitions broadly (e.g., Ajzen, 2011a; Darker et al., 2007; Elliott \& Armitage, 2009; Hardeman et al., 2002). The findings here therefore present potential ways to increase the effectiveness of public education campaigns and interventions to increase natural hazard preparation.

\section{Future Research}

Specific future ways to apply the findings of this research in interventions and education campaigns are presented more thoroughly in the General Discussion, which considers the findings from this study in context with the findings of the other studies. The results of the key test of this study, confirming that intentions to prepare for natural hazards do predict behaviour but that the effect is only moderate in strength, identifies that behaviour change tools such as goal setting and implementation intentions may be necessary to increase the translation of intentions into behaviour (e.g., Fennis, Adriaanse, Stoebe, \& Pol, 2011). These tools have already been used to an extent in the earthquake planning guide offered in this study and Study 2, produced and distributed by the Wellington Region Emergency Management Office.

The inclusion of these concepts could be made more extensive and structured in line with the relevant theories and findings. Another way to improve such campaigns is to pair a planning document with evidence-informed messaging. For example, to increase uptake of resources, agencies can convey that even those who already have some knowledge about why and how to prepare can benefit from more information, being careful not to imply that existing knowledge or efforts are futile. One way to do this could be to highlight novel information 
included in, or benefits of, such resources; someone might know what actions they can take to prepare their household, but information on how to prioritize those actions, as is included in the earthquake planning guide, could benefit them.

\section{Conclusion}

Study 3 achieved its main aims of replicating the TPB components which associate with intentions to prepare for natural hazards identified in Study 2 and demonstrating that those intentions predict subsequent preparation behaviour. Results of the final refinement to the TPB model included in this study, introducing the two-factor distinctions at the belief level, support further testing and future application of this refinement. Study 3 presents five specific, targetable beliefs which are theoretically and statistically associated with natural hazard preparation. The next step, to test an intervention targeting these beliefs, or to test adaptations to an existing intervention, is beyond the scope of this thesis. However, rather than creating an entirely new intervention, it is logical to next test whether an existing intervention impacts the factors identified in this study and so could be adapted to increase its effectiveness. One such intervention, the ShakeOut earthquake drill, is introduced and evaluated in the next chapter. New Zealand's National Emergency Management Agency worked with researchers from a number of institutions to evaluate the success of the 2018 drill; this existing evaluation programme provided an opportunity to test the usefulness of the TPB measures and framework developed so far in this thesis to evaluate an intervention. Running and testing an intervention based on the findings presented so far in thesis is left for future research. 


\section{Chapter 6: Study 4}

\section{Introduction}

\section{Overview}

Several natural hazard preparation campaigns within New Zealand as well as globally could be assessed for their efficacy using theory-informed evaluations which would produce evidence to improve the intervention. Doing so would help to prevent the redundancy of designing an entirely new intervention (e.g., Joffe, Perez-Fuentes, Potts, \& Rossetto, 2016) and would leverage the existing efforts of government organizations to increase preparedness. One such campaign is the ShakeOut earthquake drill mentioned in Chapter 1 (Perry et al., 2008). This study will examine the effects of the 2018 ShakeOut drill carried out in New Zealand on targeted and peripheral behaviours as well as on related cognitions and Theory of Planned Behaviour components. While it would have preferable within this thesis to develop and test an intervention specifically designed in line with the findings of the previous studies, such work was infeasible given the time and resource constraints. However, coincidental work to evaluate the 2018 ShakeOut drill, which sees high participation rates and has been repeatedly implemented in New Zealand and overseas, provided an opportunity to test the usefulness of the TPB measures and framework to evaluate the impacts of an earthquake preparedness campaign with relatively little time or resource cost.

Flying objects and people falling during shaking are among the leading causes of injury during earthquakes in New Zealand (Johnston et al., 2014), rather than the more highly perceived risk of building collapse ${ }^{8}$ (Johnson, Johnston, Ronan, \& Peace, 2014). Despite this risk, evidence such as CCTV footage from the 2011 Christchurch earthquake suggests that few people take protective actions during shaking (Lambie et al., 2017). While this behaviour may not reflect a lack of knowledge of the correct actions, the adoption of those actions could be increased through practice such as behavioural drills (Ronan et al., 2008).

Fire-related injuries and deaths have decreased dramatically since the United States adopted fire drills in schools in the 1960s (Johnson et al., 2014), leading the way for drills and education programmes for other hazards. Such drills and education programmes focused on

\footnotetext{
${ }^{8}$ Johnson et al.'s (2014) study tested risk perception in the United States, which has similar building codes to New Zealand. There are many developing countries where building codes are not sufficient to prevent widespread collapse, such as was seen in the 2010 Haiti earthquake where hundreds of thousands of buildings collapsed. There are therefore contexts different to the one under study here in which building collapse is an objectively high risk and a more significant cause of death and injury.
} 
earthquakes can lower children's fear while also increasing risk perception (Ronan et al., 2008). Limited evidence with other populations demonstrates the efficacy of drills to encourage actions to reduce risk (e.g., Witvorapong, Muttarak, \& Pothsiri, 2015) and improve response knowledge (e.g., Perry, 2004), as well as potentially increase the use of protective actions by addressing biases such as fatalism and beliefs that such actions will be beneficial (e.g., Ronan et al., 2008).

\section{The ShakeOut Earthquake Drill}

The state of California, in an area of the United States with many active faults, introduced the ShakeOut drill in 2008 (Perry et al., 2008). The drill included a hypothetical earthquake scenario useful for emergency management planning to demonstrate potential impacts as a means of increasing household preparation, and as a context for the earthquake drill itself. This first drill involved over five million people registering to practice "drop, cover, and hold on" as the recommended protective actions to take during earthquake shaking. The drill is now run yearly in many areas of the United States (Adams, Karlin, Eisenmann, Blakely, \& Glik, 2017), as well as in other earthquake-prone countries such as New Zealand. The 2009 ShakeOut Drill on the West Coast of New Zealand's South Island was the first such drill run in a country other than the United States (Orchiston, Manuel, Coomer, Becker, \& Johnston, 2013). The drill has since been run in New Zealand in 2012, 2015, 2018, and 2019. A specific time and date are decided for each drill and at that time, several radio stations broadcast a cue for the drill to begin. Those participating then practise "drop, cover, and hold". The National Emergency Management Agency also encourages the practice of other actions which should be undertaken following a large earthquake, including family reunification at schools and tsunami hīkoi (walk) for those in coastal areas. Finally, when participants register through the ShakeOut website (shakeout.govt.nz), they have access to information including why "drop, cover, and hold" is encouraged and ways to get more prepared.

Despite the broad implementation of drills such as ShakeOut, empirical evaluations are sparse (see Becker, 2009; Dufty, 2009; Johnson et al., 2014; Ramirez, Kubicek, Peek-Asa, \& Wong, 2009; Tipler, Tarrant, Johnston, \& Tuffin, 2016), and tend to focus on observations of logistic elements such as participation rates (e.g., Johnson et al., 2014; Orchiston et al., 2013). Research in the United States reports some improvement in knowledge after the ShakeOut drill, including correct protective actions, but highlighted that many children could not translate those actions to situations other than the one practised in the drill, such as what to do at home rather than the classroom in which they practised (Johnson et al., 2014). A qualitative interview study conducted in the United States suggests that the 2008 drill had a positive impact on 
general preparedness for earthquakes (Becker, 2009). In New Zealand, studies showed that the 2009 ShakeOut drill increased the behaviour of making a household plan (Orchiston et al., 2013) and the 2012 drill increased general preparation (Tipler et al., 2016), though again both studies focused on children and their families.

Little research has examined cognitive factors that might be affected by participation in the ShakeOut drill or considered adult samples. One exception is a United States study which used a general-sample, quantitative survey to identify impacts of the ShakeOut drill on social and cognitive factors including outcome efficacy, self-efficacy, and personal responsibility (Adams et al., 2017). This research found that participants who also engaged in other community or online activities aligned with the drill showed better preparedness knowledge and self- and outcome-efficacy.

Previous work in New Zealand has demonstrated that people who participated in the 2012 or 2015 ShakeOut drill demonstrated better knowledge of the correct protective actions to take during earthquake shaking both when inside and when outside, although knowledge of actions to take when outside overall was low (Vinnell, Wallis, Becker, \& Johnston, 2020). This study also demonstrated that people who participated drill had undertaken more additional preparation actions, in line with the types of actions examined in Studies 1, 2, and 3, than those who had not participated. While the main focus of the drill is on teaching "Drop, cover, and hold", the National Emergency Management Agency also leverages the considerable engagement with the drill to encourage other preparation actions. Further, the study by Vinnell et al. (2020) tested a limited number of cognitive factors, including fatalism and optimism biases, demonstrating some significant differences between participants and non-participants. This study aims to replicate the findings of Vinnell et al. as well as to test a larger number of cognitive factors using the theoretical framework of the Theory of Planned Behaviour to better identify potential peripheral effects of the drill.

Our understanding of the impacts of the ShakeOut drill in New Zealand, and ways to improve it, would be helped by a similar quantitative study of the general population examining impacts on cognitions, other preparation behaviours, and actual use of the taught protective actions (Becker, 2009). The present study therefore tests whether those who participated in the 2018 New Zealand ShakeOut drill score higher across the TPB components (intentions, experiential attitudes, instrumental attitudes, injunctive norms, descriptive norms, selfefficacy, and controllability), as well as general earthquake preparation, knowledge of the protective actions "drop, cover, and hold", and the use of those actions during actual earthquake shaking. This study differs from the previous studies as it examines participation in the 
ShakeOut drill throughout New Zealand, rather than focusing on Wellington. While factors such as levels of experience, cultural makeup, and exposure to previous public education campaigns and interventions do vary across New Zealand, consistency across the findings of Studies 2 and 3, as well as limited effects of demographic variables in existing literature, suggest that such variations are unlikely to meaningfully impact the findings. Further, similar to the purpose of the previous studies to identify beliefs which are broadly relevant across contexts, it is useful to examine the ShakeOut drill at the national level at which it is run.

\section{Hypotheses}

Consistent with the literature presented above, and a previous study on which this study is based (Vinnell et al., 2020), I made the following predictions. Compared to individuals who did not participated in the ShakeOut drill, those who participated in the drill would:

1. Have better knowledge of the correct actions to take during an earthquake;

2. Be more likely to have used the correct actions during an actual earthquake since the time of the drill;

3. Have undertaken more actions to prepare for earthquakes since the time of the drill;

4. Demonstrate weaker biases (i.e., lower fatalism and unrealistic optimism); and

5. Have higher scores for each of the TPB components (attitudes, norms, PBC, and intentions).

Beyond these hypotheses that focused on the impact of the drill, I also used the opportunity to examine the TPB model. Results for both the Treatment and Control groups were expected to align with those found previously in Study 2, with experiential attitudes, instrumental attitudes, and self-efficacy positively associated with intentions to prepare for earthquakes and norms and controllability either weakly or not significantly associated with intentions. This study was pre-registered on the Open Science Framework: https://osf.io/xwzey/

\section{Method}

\section{Design}

Study 4 used a quasi-experimental, between-groups design to examine whether people who had participated in the 2018 New Zealand ShakeOut drill demonstrate better knowledge, behaviour, and beliefs regarding earthquake preparation than those who did not participate. Those who did participate are designated the Treatment group while those who did not participate are termed the Control group.

This study was conducted 11 months after the date of the 2018 ShakeOut drill. This allowed for the testing of long-term effects as well as the assessment of whether participants 
had undertaken protective actions in a real earthquake since the time of the drill. Such large time delays have been used but are rare in TPB intervention evaluations (Armitage, Reid, \& Spencer, 2013). However, it is important to establish whether practising a behaviour during a drill actually increases that behaviour in the appropriate circumstances. Given the unpredictability of earthquakes, leaving a considerable window between the drill and the evaluation increases the likelihood that most participants will have experienced at least one instance of earthquake shaking.

\section{Participants}

Study 4 sampled participants from across New Zealand to align with the goals of the National Emergency Management Agency to assess the effectiveness of the drill for the entire country, since earthquakes pose a risk to all of New Zealand. Recruitment of participants in the Treatment and Control groups was conducted online using distinct strategies. Participants in the Treatment group were recruited from a registry of ShakeOut drill participants. When registering for the drill through the ShakeOut website (https://www.civildefence.govt.New Zealand/get-ready/new-zealand-shakeout-2019/), those registering are given the option to provide an email address to be contacted about the drill in the future. Only email addresses given for this purpose were used to recruit participants. Approximately 8,500 participants provided addresses. Of those, approximately 2,000 addresses belonging to schools were removed as a separate survey had already been sent to those participants. The remaining addresses were then sorted into a random order and the first 5,000 were sent a recruitment email following the process used in previous evaluations. Further, given that equal sample sizes are desirable, inviting only 5,000 of the 6,500 registered was expected to return enough participants so that this sample could be matched to the size of the non-drill sample. Sending invites to all available addresses would have resulted in more data than is practically useful. As it is important for social science research to limit the work we impose on participants (Lingler, Schmidt, Gentry, $\mathrm{Hu}, \&$ Terhorst, 2015), inviting those 1,500 people would have resulted in significant time investments from participants for no research benefit.

In order to limit the potential confound that those who participate in an earthquake drill are logically more aware of and interested in earthquakes and hazards generally than those who do not, the Control group participants were deliberately recruited through connections to earthquakes and hazards. Participants who provided their email addresses to participate in a future similar study when they completed the Study 2 survey were emailed the current survey $(N=146)$. Finally, the survey link was shared by a number of hazard-related organizations through their social media channels (e.g., regional emergency management groups). 
Nine hundred and ninety-two individuals across both groups clicked on the survey link. Of those, 162 did not proceed to participate. A further seven participants did not answer any questions beyond Question 7 (asking if they participated in the drill). These 169 participants were removed from the dataset. No participants indicated on the demographic questions that they were under the age of 18. Three participants were not in New Zealand during the drill and so were excluded from the data set and all analyses. This left 580 participants who had undertaken the ShakeOut drill and 240 that did not, making a total sample of 820 participants. In order to have roughly equal sample sizes for comparative analyses, a sub-sample of 240 was randomly selected from the drill participant group to match the non-drill participant group. Participants in these Treatment and Control groups did not significantly differ on any of the demographic variables, so the following demographics are for the combined sampled of 480 participants.

Demographics. Across the combined sample, participants' ages ranged from 19 to 89, with a mean of 51.87 ( $S D=15.03 ; 117$ participants did not provide their age), higher than the mean age in Study 1 and Study 2. This study had the same gender bias as the previous two studies with 304 women (74.9\% of those who provided their gender), 99 men (24.4\%), and 3 participants who identified as gender diverse $(0.7 \%) ; 74$ participants did not provide their gender. As with the previous studies in this research, the majority $(76.8 \%$ of those who responded) most strongly-identified as New Zealand European/Pākehā, with only 4.9\% identifying as Māori, $6.9 \%$ as European, 3.0\% as Asian, 2.7\% as North American, $0.7 \%$ as Pasifika, $0.7 \%$ as African, and $0.2 \%$ as South/Latin American. Of the 405 participants who reported their highest level of education, $4.0 \%$ had not completed secondary school, $173 \%$ had a secondary school qualification, $22.7 \%$ had a polytechnic or trade certificate, $29.6 \%$ had an undergraduate degree, $14.3 \%$ had a postgraduate degree, $11.1 \%$ had a Master's degree, and $1.0 \%$ had a Doctorate.

\section{Procedure}

The survey was delivered online using the Qualtrics platform between September $11^{\text {th }}$ and October $11^{\text {th }}, 2019$. Completion took approximately 15 minutes and was entirely voluntary. This study is part of a larger project which has been evaluated by ethics peer review through Massey University and judged to be low risk, consistent with the procedure for ethical approval of studies using human subjects required by that University. The full survey is presented in Appendix 6.

\section{Materials}


Participants were presented with several questions to identify their level of participation in the ShakeOut drill and knowledge of correct actions to undertake during shaking both inside and outside. In both instances, the correct action is to "drop, cover, and hold", moving no more than a few steps to avoid particularly dangerous hazards such as windows if inside or powerlines if outside. These questions about what to do inside and outside during shaking were open response so that participants could report what they believe to be the correct actions. Information about how these data were coded is presented in the Data Treatment section below.

Further, participants reported whether they had used "drop, cover, and hold" during a real earthquake as well as whether they had undertaken peripheral behaviours (e.g., creating an emergency plan). Participants were also asked how often they "think about earthquake issues and problems" in their community and how often they "talk about earthquake problems and issues with others" in their community, to which they could respond "Once a week or more", “A few times a month", “Once a month", "Rarely", or "Never".

Participants then responded to a number of scale-based items to assess cognitive biases and to measure the antecedents of intentions to prepare as defined in the TPB. The TPB components (instrumental and experiential attitudes, injunctive and descriptive norms, selfefficacy and controllability, and intentions) were measured using the same scales as in Study 3 except that they asked about preparing for earthquakes, rather than natural hazards, to align with the aim of the ShakeOut drill. The belief measures were omitted to limit the length of the survey and therefore increase completion rates. The scales used for key biases are presented below. Finally, participants answered demographic questions including age and gender. These questions were primarily for the purpose of ensuring there were no differences between the two comparison groups beyond those which could feasibly have been affected by the drill. Researchers and practitioners in the field $(N=6)$ were given the survey to provide any feedback on errors in the online survey construction or ways to improve the clarity of the questions.

Fatalism. Fatalism was measured with five items using a 5-point Likert response scale. The first item, taken from previous unpublished studies (Vinnell et al., 2020), asked participants to indicate their agreement, between "Strongly agree" (1) and "Strongly disagree" (5), to the statement: "Earthquakes are too destructive to bother preparing for". Four further items from McClure and Velluppillai (2013) used the same response scale for the following statements: "Earthquakes are going to cause widespread loss of life and property whether we prepare for them or not"; "If people make preparations for earthquakes they are almost certain not to work"; "There is nothing people can do about earthquakes, so there is no point trying to 
prepare for that emergency"; and "The way I look at it, nothing is going to help if there were an earthquake".

Unrealistic optimism. Unrealistic optimism was measured with six items, following the design of Spittal, McClure, Siegert, and Walkey (2005). The first two items related to preparation and asked: "How prepared do you think you are for a major earthquake?" and "Think of an acquaintance (someone you know only slightly) who lives in New Zealand. How prepared do you think they are for a major earthquake?". Participants responded to these two items on a 7-point Likert-type scale ranging from "Not prepared" (1) to "Very well prepared" (7). The other four items used the same stem which reads: "If a major earthquake were to occur in the Wellington region, how likely do you think it is that it would cause:" followed by: "harm to you?"; "damage to your property?"; "harm to the acquaintance you thought of when answering the earlier question?" and "damage to the property of the acquaintance you thought of?". These items also used a 7-point Likert type response scale, ranging from "Very unlikely" (1) to "Very likely" (7). Details of how these responses will be used is given in the Data Treatment section below.

\section{Data Treatment}

Some reverse-coding was conducted to ensure that all scale values range from low, indicating weak or negative responses, to high, indicating strong or positive responses. Responses to the questions about actions to take during shaking were coded by the lead researcher as correct or incorrect based on the protocol established and used in a previous study (Vinnell et al., 2020). Level of preparation was transformed into a single score for each participant which represents the number of reported actions out of the 31 given, in line with the procedure used in Studies 1, 2, and 3. Data from the fatalism scale and all TPB measures were used to calculate a mean score for each participant, provided they answered at least half of the items in the particular scale. A mean score for unrealistic optimism was calculated by subtracting the value given in relation to an acquaintance from that given in relation to themselves for preparation and the opposite for harm and damage. These three values were then averaged to calculate a mean score for each participant (Spittal et al., 2005). Missing data for these scales ranged from 5.5\% to $10.1 \%$. SPSS Version 25 was used for chi-squared cross tabulations and $t$ tests. Confirmatory factor analyses and path analyses were conducted in Mplus Version 8.1 (Muthén \& Muthén, 1998-2017). 


\section{Results}

\section{Hypothesis 1}

Hypothesis 1 predicted that those who participated in the ShakeOut drill would be more likely than those who did not participate to know the correct actions to take during earthquake shaking both when inside and when outside. This hypothesis was tested using chi-squared cross tabulations, comparing the ratio of correct to incorrect answers between the Treatment and Control groups. Those in the Treatment group were more likely to give a correct answer as to the actions to take when indoors during shaking (64.4\%) than to give an incorrect answer $(35.6 \%)$. As predicted, this ratio differed significantly from the ratio of correct $(42.0 \%)$ to incorrect $(58.0 \%)$ answers in the Control group, $\chi^{2}(1)=24.12, p<.001$, Cramer's $V=.23, p<$ .001 . Also as predicted, the same pattern was found for knowledge of actions to take if outside, although correct responses were lower overall. In the Treatment group, $48.5 \%$ of participants gave a correct answer, while $51.5 \%$ gave an incorrect answer. This ratio differed significantly from the ratio of correct $(24.7 \%)$ to incorrect $(75.3 \%)$ answers in the Control group, $\chi^{2}(1)=$ 29.02, $p<.001, V=.25, p<.001$.

Overall, these findings demonstrate that the ShakeOut earthquake drill achieves its main purpose of teaching what actions to take during shaking (i.e., "drop, cover, and hold"). While there is room for improvement, more participants knew to "drop, cover, and hold" outside during shaking than in previous evaluations of previous ShakeOut drills conducted in New Zealand (2012 and 2015; Vinnell et al., 2020).

\section{Hypothesis 2}

While it is important for people to know the correct protective actions to take during earthquake shaking as this is necessary for this behaviour to be performed, it is also important to evaluate whether this knowledge translates to actual performance of the behaviour during real earthquakes. Earthquake shaking is an unsettling experience and often strong emotions such as fear and panic can lead to people not responding in the ways that they know they should (Lambie et al., 2017). As with Hypothesis 1, a chi-squared cross tabulation tested whether the use of "drop, cover, and hold" in a real earthquake differed between the Treatment and Control groups. Participants could report using "drop, cover, and hold in all earthquakes, no earthquakes, or some earthquakes. Those participants who had not felt an earthquake since the time of the drill, and therefore could not either undertake or not undertake "drop, cover, and hold", were excluded from this analysis.

As predicted, those in the Treatment group were more likely to have employed "drop, cover, and hold" in all earthquakes $(51.1 \%)$ than to have either employed it in some quakes but 
not others $(22.3 \%)$ or to have not employed it during shaking (26.6\%). This ratio differed significantly from that in the Control group, where the majority (66.4\%) had not used "drop, cover, and hold" in a real earthquake compared to $16.8 \%$ who had employed it in all or some earthquakes they had felt, $\chi^{2}(2)=37.17, p<.001, V=.30, p<.001$. These results support Hypothesis 2 and indicate that participating in the ShakeOut drill not only improves knowledge of the correct protective actions but also that those who know these actions are more likely to implement them during earthquakes. This translation from knowledge gained in the ShakeOut drill to correct behaviour during actual earthquakes is critical for supporting the use of ShakeOut and has not been established previously.

\section{Hypothesis 3}

Hypothesis 3 predicted that participants in the Treatment group would carry out more preparation actions following the drill than the Control group. However, the number of actions taken by those in the Treatment group $(M=1.73, S D=2.94)$ and the Control group $(M=1.77$, $S D=3.45)$ since the time of the drill did not differ significantly, $t(350)=.12, p=.908$. This non-significant finding may be explained by the fact that the Treatment group reported having already done significantly more of the actions before the time of the drill $(M=15.51, S D=$ 8.35) than the Control group $(M=13.11, S D=7.94 ; t(424)=3.04, p=.002, d=0.30)$. Therefore, the fraction of actions participants took out of the actions they had not already performed was calculated and compared, with scores ranging between 0 and 1 . The average fraction of possible actions taken did not significantly differ between the Treatment $(M=.098$, $S D=.16)$ and Control groups $(M=.084, S D=.15 ; t(345)=.89, p=.376)$.

Interestingly, participants in the Treatment group reported higher levels of general earthquake preparedness $(M=4.63, S D=1.32)$ than those in the Control group $(M=3.99, S D$ $=1.42 ; t(455)=5.03, p<.001, d=0.47)$. It is therefore possible that practising "drop, cover, and hold" makes people feel more prepared for earthquakes, even if they have not carried out any further preparations. This suggestion is supported by the Treatment group reporting that the ShakeOut was more influential on their preparedness $(M=3.56, S D=.96)$ than those in the Control group $(M=3.12, S D=1.10 ; t(338)=4.02, p<.001, d=0.44)^{9}$. In comparison, the two groups gave similar mean ratings of other potential sources of influence on their preparedness (an experienced earthquake, $p=.474$; an earthquake in which someone the participant knew

\footnotetext{
${ }^{9}$ This comparison includes only those participants in the Control group who knew about the ShakeOut drill at the time of the drill occuring $(n=192)$.
} 
was involved, $p=.344$; and an earthquake which the participant observed in the media, $p=$ $.131)$.

\section{Hypothesis 4}

Hypothesis 4 predicted that those who participated in the ShakeOut drill would demonstrate lower fatalism and unrealistic optimism biases. These biases are typically seen as barriers to preparing (McClure, Allen, \& Walkey, 2001; Spittal et al., 2005). This hypothesis was tested using independent samples $t$-tests, comparing mean fatalism and unrealistic optimism scores between the Treatment and Control groups.

The mean score for fatalism in the Treatment group $(M=4.22, S D=.54)$ differed significantly from the mean score in the Control group $(M=4.06, S D=.63 ; t(455)=2.89, p=$ $.004, d=0.27$ ), where a higher mean is "more positive" and indicates a weaker bias. Therefore, and as predicted, those in the Treatment group exhibited a lower fatalism bias than those in the Control groups. The mean score for unrealistic optimism was calculated by averaging the result of subtracting the participants' perception of an acquaintance's preparedness from their own (as optimism bias should lead to them seeing themselves as more prepared) and the result of subtracting participants' perceptions of their own likelihood of harm and damage from the likelihood of harm and damage to an acquaintance. The scores for this mean could therefore range from -7 to +7 . In contrast to the fatalism finding, those in the Treatment group demonstrated a stronger unrealistic optimism bias $(M=.44, S D=.82)$ compared to those in the Control group $(M=.29, S D=.76 ; t(454)=2.08, p<.038, d=0.20)$, where higher means indicate a stronger bias. The means for both groups suggests that overall participants had low optimism biases, although scores differed significantly from the scale midpoint of 0 for both those who participated in the drill, $t(230)=8.15, p<.001, d=.54$, and those who did not participate, $t(224)=5.64, p<.001, d=0.38$.

The Treatment group perceived themselves as more prepared than an acquaintance $(M$ $=1.23, S D=1.61)$ than did the Control group $(M=.79, S D=1.59 ; t(453)=2.96, p=.003, d$ $=0.28$ ). The two groups did not differ significantly on harm bias, $p=.48$, or damage bias, $p=$ .76. This difference in perceived preparedness could be due to a lack of bias in the Treatment group, as this group has participated in the ShakeOut drill which aims to prepare them for an earthquake whereas the Control group did not. Overall, therefore, unrealistic optimism does not seem apparent within either of the groups for harm or damage bias, and the difference in preparedness perceptions likely reflects reality rather than a bias. 


\section{Hypothesis 5}

More importantly to the overall goal of this research, Hypothesis 5 predicted that participants in the Treatment group would demonstrate higher (i.e., more positive) scores for each of the TPB components (experiential and instrumental attitudes, injunctive and descriptive perceived norms, controllability and self-efficacy, and intentions to prepare) compared to those in the Control group. This hypothesis was tested using independent samples $t$-tests, comparing mean TPB scores between the Treatment and Control groups. This hypothesis was also tested with a more stringent analysis using structural equation modelling (SEM) to compare latent mean scores using Mplus. To do so, measurement invariance was first established between the two groups for each of the individual constructs (see Byrne, 2012; Gregorich, 2007; Fischer \& Karl, 2019; Milfont \& Fischer, 2010; Vandenberg \& Lance, 2000). First, configural invariance was established. Configural invariance refers to whether the underlying structure of the models vary significantly from each other. If the structures vary, then it is not appropriate to compare them directly using a multigroup SEM. Second, metric invariance was established. Metric invariance testing checks if the factor loadings are equivalent between the two groups; that is, whether the relationship between the observed variables and the latent construct are similar in direction (i.e., positive or negative) and strength between the two groups. The establishment of metric invariance allows for the comparison of factor invariances between groups but not a comparison of factor means. Doing so required establishing scalar invariance by testing whether the intercepts differed between the two groups, considering not just the direction and strength of the factor loadings but the actual response values given to the items.

Results of the configural, metric, and scalar invariance testing is presented in Table 6.1. The chi-squared statistic was divided by the degrees of freedom to give a ratio. Following the guidelines of Vandenberg and Lance (2000) invariance was established at each level (configural, metric, and scalar) if the chi-squared ratio was below 3, the Comparative Fit Index (CFI) was above .90, and the Root Mean Square Error of Approximation (RMSEA) and the Standardized Root Mean Squared Residual (SRMR) were equal to or below .08. There were three instances when model fit was deemed poor or marginal (i.e., poor fit according to at least two of three indicators or very poor fit according to one indicator and where the Mplus program could suggest a modification to improve model fit): metric invariance in experiential attitudes, configural invariance in controllability, and configural invariance in intentions. Two items within each of these scales were allowed to correlate with each other based on the modification indices (see Table 6.1 for details). Overall, both groups demonstrated equivalent structures, 
Chapter 6: Study 4

factor loadings, and responding patterns, supporting the appropriateness of comparing latent means (Vandenberg \& Lance, 2000). 
Table 6.1

Results of measurement invariance testing between the Treatment and Control conditions.

\begin{tabular}{|c|c|c|c|c|c|c|c|c|c|}
\hline Cognition & Factor & Invariance & $\chi^{2}$ & $\mathrm{df}$ & $p$ value & Ratio & $\mathrm{CFI}$ & RMSEA & SRMR \\
\hline \multirow[t]{7}{*}{ Attitudes } & \multirow[t]{3}{*}{ Instrumental } & Configural & 7.73 & 4 & .102 & 1.93 & .974 & .065 & .023 \\
\hline & & Metric & 8.80 & 7 & .287 & 1.26 & .988 & .034 & .091 \\
\hline & & Scalar & 12.03 & 10 & .283 & 1.20 & .986 & .030 & .098 \\
\hline & Experiential & Configural & 27.23 & 8 & .001 & 3.40 & .909 & .104 & .072 \\
\hline & \multirow{3}{*}{ Item 4 with 1} & Control group & 14.81 & 7 & .039 & 2.16 & .963 & .071 & .057 \\
\hline & & Metric & 15.30 & 6 & .018 & 2.55 & .956 & .084 & .056 \\
\hline & & Scalar & 24.84 & 9 & .003 & 2.76 & .925 & .089 & .064 \\
\hline \multirow[t]{6}{*}{ Norms } & \multirow[t]{3}{*}{ Injunctive } & Configural & 2.85 & 4 & .583 & 0.71 & 1.00 & .000 & .009 \\
\hline & & Metric & 3.64 & 7 & .820 & 0.52 & 1.00 & .000 & .031 \\
\hline & & Scalar & 11.07 & 10 & .352 & 1.11 & 1.00 & .022 & .051 \\
\hline & \multirow[t]{3}{*}{ Descriptive } & Configural & 8.77 & 4 & .067 & 2.19 & .991 & .073 & .014 \\
\hline & & Metric & 11.86 & 7 & .105 & 1.69 & .991 & .056 & .035 \\
\hline & & Scalar & 14.97 & 10 & .133 & 1.50 & .991 & .047 & .040 \\
\hline \multirow[t]{7}{*}{ PBC } & \multirow[t]{3}{*}{ Self-efficacy } & Configural & 9.28 & 4 & .055 & 2.32 & .971 & .078 & .030 \\
\hline & & Metric & 12.95 & 7 & .073 & 1.85 & .967 & .062 & .057 \\
\hline & & Scalar & 18.85 & 10 & .042 & 1.89 & .951 & .064 & .056 \\
\hline & Controllability & Configural & 16.29 & 4 & .003 & 4.07 & .922 & .119 & .046 \\
\hline & \multirow[t]{3}{*}{ Item 3 with 2} & Control group & 2.66 & 3 & .447 & 0.89 & 1.00 & .000 & .020 \\
\hline & & Metric & 16.17 & 6 & .013 & 2.70 & .935 & .088 & .081 \\
\hline & & Scalar & 23.69 & 9 & .005 & 2.63 & .906 & .086 & .110 \\
\hline \multirow[t]{4}{*}{ Intentions } & & Configural & 13.90 & 4 & .008 & 3.48 & .974 & .106 & .020 \\
\hline & \multirow[t]{3}{*}{ Item 3 with 2} & Treatment group & 4.94 & 2 & .085 & 2.47 & .992 & .081 & .011 \\
\hline & & Metric & 9.44 & 5 & .093 & 1.89 & .988 & .063 & .064 \\
\hline & & Scalar & 14.59 & 8 & .068 & 1.82 & .982 & .061 & .052 \\
\hline
\end{tabular}


Table 6.2

Differences in mean scores for TPB components between those who did ("Treatment") and those who did not ("Control") participate in the ShakeOut earthquake drill.

\begin{tabular}{|c|c|c|c|c|c|c|c|}
\hline & & Treatment & & Control & & & \\
\hline Cognition & Factor & Mean & $S D$ & Mean & $S D$ & Difference between means & Latent means comparison \\
\hline \multirow[t]{2}{*}{ Attitude } & Experiential & 4.44 & 1.04 & 4.14 & .97 & $t(434)=3.12, p=.002, d=.30$ & $.31(.13), p=.022$ \\
\hline & Instrumental & 6.61 & .77 & 6.40 & .92 & $t(411.29)=2.69, p=.008, d=.27$ & $.24(.11), p=.032$ \\
\hline \multirow[t]{2}{*}{ Norms } & Injunctive & 5.52 & 1.19 & 5.20 & 1.20 & $t(441)=2.86, p=.004, d=.27$ & $.32(.11), p=.003$ \\
\hline & Descriptive & 4.54 & 1.38 & 3.99 & 1.27 & $t(440.65)=4.34, p<.001, d=.41$ & $.40(.10), p<.001$ \\
\hline \multirow[t]{2}{*}{ PBC } & Self-efficacy & 5.87 & .86 & 5.48 & 1.05 & $t(435)=4.28, p<.001, d=.41$ & $.49(.14), p<.001$ \\
\hline & Controllability & 6.15 & .87 & 5.96 & 1.01 & $t(434)=2.11, p=.036, d=.20$ & $.25(.14), p=.084$ \\
\hline Intentions & & 5.75 & 1.23 & 5.29 & 1.27 & $t(443)=3.93, p<.001, d=.37$ & $.42(.11), p<.001$ \\
\hline
\end{tabular}


The results of the mean comparisons are presented together in Table 6.2. The latent mean comparisons largely agreed with the independent samples $t$-tests: the Treatment group demonstrated higher instrumental attitudes, experiential attitudes, injunctive norms, descriptive norms, self-efficacy, and intentions to prepare than the Control group. This finding implies that those who participated in the ShakeOut drill have more positive attitudes, perceptions of norms, perceptions of control, and intentions to prepare than those who did not participate in the ShakeOut drill. However, the comparison for controllability was non-significant. This suggests that perceptions of control over preparing is not a factor in why people do or do not participate in the drill.

Hypothesis 5 is therefore largely supported. The finding of measurement invariance across the two groups for the TPB variables suggests that the differences in means are not a result of overarching differences in the pattern of responding, rather that the groups hold different attitudes and perceptions and that this distinction is likely due to participation in the ShakeOut drill. Alternatives to this explanation, including the potential confound of selfselection bias, is elaborated in the Discussion section.

\section{Exploratory Analyses}

Several analyses were planned to test for effects which are logical or informative, but for which not enough research currently exists to make predictions. This included comparing TPB models between the Treatment and Control groups, which was planned as a multi-group SEM. However, fewer participants than anticipated completed the survey (particularly those who had not participated in the drill), meaning that the samples are not large enough for full multi-group SEM, including all observed variables, which requires considerable power (Breitsohl, 2018). Therefore, the latent means calculated for the comparisons above were saved in Mplus and entered as observed variables for each of the TPB factors, as doing so reduced the issue of limited power for multi-group SEM and invariance findings provided evidence of the psychometric properties of the scales for both groups. Unlike Study 2, because the items used for the Treatment and Control groups were identical, full measurement invariance was established (see above). The models for the two groups could therefore be compared statistically, as described below, rather than visually as was necessary in Study 2.

Multi-group SEM. The structure of the paths between the TPB cognition components and intentions to prepare was compared between the Treatment and Control groups. At the first step, the paths in each group were allowed to vary freely to establish a baseline. With variance freely estimated, the model fit the data well, $\chi^{2}(12)=134.35, p<.001$, RMSEA $=.00, \mathrm{CFI}=$ $1.00, \mathrm{SRMR}=.00$. At the second step, paths were fixed across both the Control and Treatment 
groups to test whether the TPB variables explain intentions to prepare in the same way in both groups. This constrained model had good fit to the data, $\chi^{2}(6)=7.27, p=.30$, RMSEA $=.031$, $[.00-.098], p=.60, \mathrm{CFI}=.99, \mathrm{SRMR}=.038$. However, the change in CFI of -.01 is at the recommended threshold for rejecting the assumption of invariance between the two models (Cheung \& Lau, 2011; Putnick \& Bornstein, 2016), while the change in both RMSEA and SRMR surpass the threshold for change of -.015 (Putnick \& Bornstein, 2016). While each of these different fit statistics have different sensitivities (Fischer \& Karl, 2019), changes across all three demonstrating significant loss of fit suggests that the models for the two groups should not be assumed to be the equivalent. Therefore, the estimated paths for the Treatment group and the Control group are presented below (Table 6.3) using the results from the unconstrained model, when neither were fixed.

Table 6.3

Results of structural equation modelling of associations between TPB components and intention for those who did ("Treatment") and those who did not ("Control") participate in the ShakeOut earthquake drill.

\begin{tabular}{|c|c|c|c|c|c|c|c|c|c|c|c|}
\hline \multirow{3}{*}{$\begin{array}{l}\text { Cognition } \\
\text { Attitude }\end{array}$} & \multirow{3}{*}{$\begin{array}{l}\text { Factor } \\
\text { Instrumental }\end{array}$} & \multicolumn{6}{|c|}{ Treatment } & \multicolumn{4}{|c|}{ Control } \\
\hline & & \multirow{2}{*}{$\begin{array}{c}\beta \\
.18\end{array}$} & \multirow{2}{*}{$\begin{array}{l}\mathrm{SE} \\
.093\end{array}$} & \multicolumn{2}{|c|}{$\mathrm{CI}$} & \multirow{2}{*}{$\begin{array}{c}p \\
.047\end{array}$} & \multirow{2}{*}{$\begin{array}{c}\beta \\
.40\end{array}$} & \multirow{2}{*}{$\begin{array}{c}\text { SE } \\
.067\end{array}$} & \multicolumn{2}{|c|}{$\mathrm{CI}$} & \multirow{2}{*}{$\begin{array}{c}p \\
.00\end{array}$} \\
\hline & & & & .002 & .366 & & & & .267 & .529 & \\
\hline & Experiential & .045 & .072 & -.096 & .186 & .53 & .11 & .056 & .002 & .222 & .043 \\
\hline \multirow[t]{2}{*}{ Norms } & Injunctive & .28 & .092 & .095 & .455 & .003 & .20 & .071 & .056 & .334 & .006 \\
\hline & Descriptive & .11 & .070 & -.023 & .251 & .10 & .050 & .064 & -.075 & .175 & .44 \\
\hline \multirow[t]{2}{*}{ PBC } & Self-efficacy & .12 & .075 & -.028 & .266 & .11 & .22 & .096 & .034 & .410 & .020 \\
\hline & Controllability & .11 & .088 & -.067 & .279 & .23 & -.065 & .083 & -.228 & .098 & .44 \\
\hline Model $R^{2}$ & & .33 & .067 & & & .00 & .43 & .060 & & & .00 \\
\hline
\end{tabular}

Note. $\mathrm{SE}=$ Standard error. $\mathrm{CI}=$ Confidence interval. Left column is low end and right column is high end of the $95 \% \mathrm{CI}$. 
Both models significantly explained variance in intentions, $p<.001$. The model for the Treatment group explained approximately $31 \%$ of the variance in intentions, based on the adjusted $R^{2}$ value, while the model for the Treatment group explained $41 \%$ of the variance. Further, several paths within the models differed in terms of their significance; namely, the paths between experiential attitudes and self-efficacy and intentions were significant in the Control group but not significant in the Treatment group. Further, the estimate for the path between instrumental attitudes and intentions appears stronger in the Control group than the Treatment group, while the injunctive norm path appears weaker. To explore these differences, Wald tests compared a path in one model to its equivalent in the other model. Despite the apparent differences, none of the Wald tests were significant ( $p$ values ranged from .12 to .66), suggesting that overall intentions to prepare are explained similarly between those in the Treatment group and the Control group. It could be that each of the differences between individual paths in each model are too small to reach statistical significance in the Wald tests but that the overall models are different when considering all of those small path differences. A further possibility is that the sample size is too small for the Wald test to be accurate (Ohtani, 1984; von Davier, 2003). These findings therefore suggest that there may be differences in how the TPB components explain the intentions to prepare of those who have and those who have not participated in the ShakeOut drill but that further research to establish the existence and clarify the importance of any differences is advisable. Regardless, the findings provide additional support for the usefulness of the TPB in explaining intentions to carry out household preparation for earthquakes.

Critical awareness, information seeking, and correlation between fatalism and instrumental attitudes. The frequency with which participants report that they think and talk about earthquake problems and issues within their community was also compared between groups. These items approximate measures of critical awareness (Paton, Anderson, Becker, \& Peterson, 2015) and were correlated significantly and positively with a strong effect, $r=.645$, $p<.001^{10}$, so were averaged to create a single critical awareness mean score. This mean was then compared between the two groups using an independent samples $t$-test. In this test, a lower mean indicates higher critical awareness. Those in the Treatment group showed significantly more critical awareness $(M=3.12, S D=.92)$ than those in the Control group $(M=3.39, S D=$ $.99 ; t(465)=3.08, p=.002, d=.29)$, indicating that those who participated in the ShakeOut

\footnotetext{
${ }^{10}$ This correlation is for the combined sample; the correlation for the Treatment group, $r=.63, p<$ .001 , did not significantly differ from the correlation for the Control group, $r=.65, p<.001, z=.37, p$ $=.71$.
} 
drill thought and talked about earthquake problems faced by their community more than those who did not participate in the ShakeOut drill.

Participants were also asked if they had sought information about earthquakes or preparing for emergencies since the ShakeOut drill. While the key aim of the drill is to teach the "drop, cover, and hold" action, effort is also made to encourage participants to increase their awareness and knowledge of their natural hazard risks and what they can do in response to those risks. A chi-squared cross tabulation demonstrated that the ratio of participants in the Treatment group who had sought information (63.8\%) compared to those who had not $(36.2 \%)$ significantly differed from the ratio of participants in the Control group who had (36.8\%) and had not $(63.2 \%)$ sought information, $\chi^{2}(1)=33.76, p<.001$.

Finally, fatalism scores were correlated with instrumental attitudes. Instrumental attitudes are not typically studied in the disaster field but are closely approximated by outcome expectancy and response-efficacy which are used interchangeably in the field (Becker et al., 2015; Johnston et al., 2013). Similarly, these concepts of outcome expectancy and responseefficacy have been equated with fatalism (Becker et al., 2015; Johnston et al., 2013). Fatalism is an important and often-studied predictor of lack of preparation actions (e.g., McClure et al., 2001; Paton, Kershoult, \& Skinner, 2017), so evidence of strong overlap with instrumental attitudes would provide further support for the application of the TPB to natural hazard research. These two concepts are significantly and positively correlated in this study, and the strength of the association is moderate, $r=.375, p<.001^{11}$. This finding suggests that there is some similarity in the latent constructs being measured here by the instrumental attitudes and the fatalism scales, but that there is sufficient difference between the two constructs for them to continue to be considered separately. It is possible that this lack of overlap in these constructs is because questions measuring fatalism are more targeted towards the nature of the hazard (e.g., "Earthquakes are too destructive to bother preparing for") whereas the questions measuring instrumental attitudes directly refers to a behaviour (preparing; e.g., "I think that preparing for earthquakes is useless/useful").

\section{Discussion}

One of the leading causes of injury from earthquakes (in countries with strong building codes such as New Zealand) is people being struck by falling objects or being thrown off their feet (Johnston et al., 2014). Teaching simple protective actions to take during earthquake

\footnotetext{
${ }^{11}$ This correlation is for the combined sample; the correlation for the Treatment group, $r=.36, p<$ .001 , did not significantly differ from the correlation for the Control group, $r=.37, p<.001, z=.12, p$ $=.91$.
} 
shaking can therefore reduce the number of injuries caused by earthquakes (Johnston et al., 2014). Several authors have called for better evaluation of hazard education campaigns (e.g., Becker, 2009; Dufty, 2009) including earthquake and tsunami drills (e.g., Johnston et al., 2016). This study provides evidence that the ShakeOut drill has positive effects for both its primary goal of teaching protective actions to take during shaking and its secondary goal of improving preparation-related thoughts and biases. However, this evaluation did not find that the 2018 ShakeOut drill increased participants subsequent preparedness beyond the use of the "drop, cover, and hold" action. This section briefly summarizes the main results of Study 4, discusses implications for these findings for both future ShakeOut drills as well as other public education campaigns, and then discusses several important limitations of this study. Little research has evaluated the ShakeOut drill to this extent, increasing the importance and usefulness of the present findings.

\section{Summary of Results}

Hypotheses 1 and 2, relating to the primary goal of the ShakeOut drill, were both supported. Those who participated in the drill were more likely than those who did not to know the correct protective actions to take during shaking both inside and outside and to have used those protective actions during real earthquake shaking. Even in the Treatment group, however, just under half of the participants knew what to do if they are outside during earthquake shaking. This finding shows that the drill is effective but could be improved

One key reason for the long delay between the drill and the evaluation survey was to increase the feasibility of the latter analysis. While teaching "drop, cover, and hold" is the primary goal of ShakeOut, it is crucial to know whether the way in which the drill teaches these actions is effective at increasing actual use rather than assuming that it does so (Johnston et al., 2011). Because earthquakes are unpredictable, leaving a larger window of time greatly increases the likelihood that participants will have experienced shaking in which to use "drop, cover, and hold", allowing for the crucial comparison of the proportion of people who have employed these actions between the Treatment and Control groups. The evidence found here supporting the assumption that the drill does increase the use of "drop, cover, and hold" in earthquakes is crucial for justifying the continued use of the drill; this benefit therefore outweighs the cost of using such a long delay, which is discussed in the following limitations section. Future studies could evaluate impacts closer to the time of drill, potentially as well as at a later time such as the one used here to provide further support for long term effects.

Hypothesis 3 concerning differences in preparation actions between the two groups was not supported. While participants in the Treatment group self-reported higher general 
preparedness than did the Control group, when asked about specific actions taken since the time of the drill there was no difference between the two groups, even when accounting for the number of past actions. Importantly, the number of actions already taken by participants before the time of the drill differed significantly between the Treatment and Control group. This difference suggests that people who are more prepared might also be more willing to engage in the ShakeOut drill, leading to a possible self-selection bias. The implications of this bias, and ways to limit it in future, are discussed in the following limitations section.

Although not formalized as hypotheses, other specific behaviour-related variables were measured separately and compared between groups. Those who had participated in the drill reported higher critical awareness than those in the Control group, indicating that they more frequently think about the challenges posed by earthquakes and talk about those challenges with their community. Further, those who had participated in the drill were more likely to have sought extra information about preparing for earthquakes than those who did not participate in the drill. This finding suggests that even if the drill does not motivate extra preparation actions, it might encourage participants to seek out information on the risks they face and what they can do in response to those risks. Both risk awareness and knowledge of preparation behaviours are necessary for preparation to occur (e.g., Ballantyne, Paton, Johnston, Kozuch, \& Daly, 2000), so this finding about information seeking suggests another benefit of the ShakeOut drill.

Hypothesis 4, concerning impacts of participating in the ShakeOut drill on cognitive biases, was partly supported. Participants in the Treatment group demonstrated weaker fatalism biases than did those in the Control group, suggesting that participating in an earthquake drill might make preparing for earthquakes seem more feasible. Those who had participated in the ShakeOut drill demonstrated higher unrealistic optimism than those who had not participated, although this was driven by participants feeling they were more prepared than an average acquaintance. This difference likely does not reflect a bias, however, as learning "drop, cover, and hold" is an important step to increase preparedness so those who have learned and practised these actions (i.e., the Treatment group) might be expected to feel more prepared than those who have not learned and practised these actions (i.e., the Control group).

Finally, and more importantly for the theoretical goals of this research, Hypothesis 5 concerning differences in mean scores on the TPB components was also largely supported. The Treatment group scored higher on six out of seven of the measured components of the TPB: experiential and instrumental attitudes, injunctive and descriptive perceived social norms, selfefficacy, and intentions to prepare. These findings show that those who participated in the ShakeOut drill see preparing for earthquakes as a more positive experience and more 
beneficial, that others similar to them approve of preparing and have prepared, and that they are more capable of preparing than those who did not participate in the drill. Because the study did not include random assignment, it is not possible to conclude based on the data if these differences are due to impacts of the drill itself or differences in the type of people who do versus do not participate, although the establishment of measurement invariance between the two groups provides support for the suggestion that the differences reflect impacts of the drill rather than group differences. Future research could test changes in TPB components as a result of participating in the drill using either a pre-test post-test design or random assignment (or, ideally, both).

As well as suggesting that participating in the ShakeOut drill can improve several important cognitive factors relating to preparation behaviour, these findings show the usefulness of the TPB as a tool to evaluate the effects of a behaviour change intervention. Further, there were several differences in which of the TPB components were associated with intentions between the two groups, and a larger amount of variance was explained in the model for the Control group, which included more significant associations. However, the two models imply that targeting instrumental attitudes and injunctive norms is most appropriate as these were the only two components which significantly associated with intentions to prepare for both the Treatment and Control groups. Targeting these two factors could help to increase people's intentions to prepare which is likely in turn increase their actual preparation. Of these two (instrumental attitudes and injunctive norms), injunctive norms present a better target as the lower mean score offers more opportunity for such norms to increase (McKenzie-Mohr, 2011).

\section{Implications and Applications}

While the ShakeOut earthquake drill has been evaluated before, both internationally and within New Zealand (e.g., Johnson et al., 2014; Johnston et al., 2011; Orchiston et al., 2013), these evaluations tend to focus on logistics of running the drill such as how many people participate. Some of these studies have also evaluated whether earthquake drills increase knowledge of protective actions (Johnson et al., 2014), along with other important factors such as response capacity and negative emotional responses (Johnston et al., 2011). While some of these previous studies show that earthquake drills increase preparation actions, these typically assume that participants who are observed to undertake the correct behaviour during the drill have correct knowledge and understanding of those behaviours (Johnson et al., 2014). Further, many previous studies focus on the drill in schools which can target parents and households through children's homework (Dufty, 2009; Tipler et al., 2016) or individual communities 
(Witvorapong et al., 2015). The current findings demonstrate that the New Zealand ShakeOut drill could be made more effective at encouraging other earthquake and tsunami preparation actions among the general public.

The positive findings regarding differences in biases as well as TPB factors show ways in which the drill could be improved to increase both its impact on these factors and on preparation behaviour. For example, the finding that perceived injunctive norms are positively associated with intentions to prepare for earthquakes suggests that messaging could be included to emphasize that preparation is approved of within New Zealand. Already, the ShakeOut campaign has used several high-profile figures as support, including film-makers Sir Peter Jackson and Sir Richard Taylor and Prime Minister Jacinda Ardern. A simple yet potentially effective change would be to ask these influential figures to also "endorse" preparing for earthquakes and tsunami.

This study supports the capability of the TPB to inform a public education campaign. Different factors were found to associate with intentions than were found in the previous studies in this thesis, showing that the theory can identify specific factors which are more relevant in different behaviour contexts. Given that public education campaigns and behaviour change interventions typically have logistic restrictions around time, effort, and cost, identifying one or two factors around which to tailor the intervention provides a similar benefit to identifying multiple factors as it is likely that only one or two would be selected for targeting anyway (McKenzie-Mohr, 2011). Further, the similarity in which TPB factors were associated with intentions to prepare, particularly in the control group, to the findings of Study 2 and Study 3 suggests that the findings in the earlier studies, among residents of the Wellington Region and referring to natural hazards, are generalizable to New Zealand more broadly and different target hazards. This similarity provides further support that the factors identified in this thesis as relevant to preparation are likely to be relevant in other, similar contexts internationally (e.g., countries with similar placement on cultural dimensions such as individualism and where the importance of factors such as self-efficacy have already been established).

\section{Limitations and Future Directions}

Notwithstanding the novel contributions of this study, there are limitations worth noting. First, there are several instances where participants' responses may be skewed by selfserving biases where they respond to questions in such a way as to make themselves appear "better" to the researcher (Fisher, 1993). For example, it is possible that some participants reported that they used "drop, cover, and hold" in a real earthquake when they in fact did not because believe the researcher will judge them less favourably. There is, however, little reason 
to expect that this bias would affect the groups differently, so comparisons are still meaningful. Further, although self-reports of behaviour are limited in this way, they are often the most feasible, if not the only possible, way to measure behaviour. Given the unpredictability of earthquakes, it would be impossible to observe participants' actual behaviour during real shaking even if resources were available for such intensive data collection. Previous research has used CCTV footage (e.g., Lambie et al., 2017), but this does not easily allow for establishing whether the individuals in the footage had participated in the drill. Self-reports are therefore commonly used; their inherent limitations are acknowledged but not considered as sufficient to undermine the findings.

Second, a delay of approximately 11 months between the drill and the evaluation survey creates the possibility that participants will not accurately recall behaviours such as the actions that they had done before the drill. The finding of significant differences between those who did and did not participate in the drill suggests that the positive effects of participation persist for a relatively long time. The ShakeOut drill is currently conducted yearly in New Zealand; the results found here suggest that running the drill every year does have benefits for those who participate which last for the entire time between drills. However, it is possible that knowledge of correct actions was higher shortly after the drill and had decreased by the time of this evaluation. Future research could test effects over a shorter time as well as at a similar delay of approximately a year to identify whether the impacts of the drill lessen over time and therefore whether the drill should be run more frequently.

Further, as mentioned above, such a delay is necessary for perhaps the most important test within this evaluation. The drill assumes that those who know and practise "drop, cover, and hold" in a non-earthquake situation will employ these actions during an actual earthquake. Support for this assumption as found here, therefore, is crucial for supporting the continued running of the ShakeOut drill. More research around understanding whether people are able to use protective actions in situations other than the one in which they practised them is important to identify whether the drill needs to be changed to include more information on the "why" of the actions so that people can alter them if necessary to fit their situation (Johnston et al., 2016).

Finally, and perhaps most critically, the possibility of an overall self-selection bias cannot be ignored. It is possible that the differences in biases and TPB factors could be because those who participated in the ShakeOut drill participated because they hold different beliefs, attitudes, perceptions of norms, and perceptions of self-efficacy. Evidence for this selfselection bias is demonstrated in the higher number of preparation actions done before the time of the drill by those in the Treatment group compared to the Control group. However, although 
this difference is statistically significant, it is not large. Further, the participants did not differ on any demographic factors which can be associated with earthquake-related beliefs and behaviour such as age and gender (e.g., Becker et al., 2015).

Although random assignment is uncommon in natural hazard behaviour intervention studies (Bradley, McFarland, \& Clarke, 2014 found only one out of 27 studies), steps were taken to reduce the possibility of self-selection. Those participants who were recruited through social media were targeted via groups related to earthquake communication or emergency management, so that anyone who found the link via those channels did so because they were following those groups and therefore are already engaged with earthquake and hazard preparedness. Regardless, the majority of the Control group came from the 5,000 people who were invited to participate because they had registered on the ShakeOut website as intending to participate ( $80 \%$ of the Control group knew about the drill at the time it occurred, and $70 \%$ had registered to participate). Given this sampling strategy there should be few, if any, confounding differences between the samples that existed prior to their participation.

Assuming that there were existing differences which led to the significant findings above, however, leads to similar conclusions about ways to improve the drill. Those who registered but did not participate would have had to engage with the campaign to some extent, so they received similar information as did those who participated. Assuming all else is the same, and that the findings above do not represent effects of the drill, those findings would then imply that those who have weaker biases and more positive attitudes, norm perceptions, self-efficacy perceptions, and intentions to prepare were more likely to continue to proceed to participate in the drill. Targeting these factors in the communication before the drill could therefore increase the number of people who participate, particularly from among those registered but also within the public who have not registered. Interpreting the results in this way, assuming that the group differences are a result of self-selection bias, therefore leads to similar implications for changes to the ShakeOut campaign.

Further, this evaluation was undertaken as part of a wider and ongoing collaboration with the National Emergency Management Agency to evaluate the New Zealand ShakeOut drill. This survey was based on surveys from 2012 and 2015 and was required to follow the methodology used previously as closely as possible, which included the recruitment strategy and the delay between drill and evaluation. Alternatives, such as random representative sampling and pre- and post-test designs were beyond what was possible with the time and resources available within the project and would have been difficult to justify without preliminary evidence that such methods would reveal useful information. The above findings 
provide that preliminary evidence and demonstrate that more rigorous methodologies would be valuable to more clearly and confidently understand the effects of the ShakeOut drill. This improvement to the survey strategy is a key direction for future evaluations of the ShakeOut drill as well as for other evaluations of public education campaigns.

\section{Conclusion}

This study demonstrates that those who participated in the ShakeOut drill had more correct knowledge of protective actions and were more likely to have taken those actions in a real earthquake than those who had not participated in the drill. Further, and more importantly for this thesis, ShakeOut participants scored higher across all TPB components (except controllability), showing more positive attitudes, perceptions of norms, self-efficacy, and intentions to prepare than those who did not participate in the drill. There are limitations in the methodology of the study, but these findings support the continued running of the ShakeOut drill both in New Zealand and internationally while also identifying ways in which the drill can improved. Further, this study supports the application of the TPB as a tool to evaluate the impacts of interventions aiming to change behaviour relating to natural hazards 


\section{Chapter 7: General Discussion}

\section{Chapter Overview}

This chapter presents a summary and discussion of the research presented in this thesis. First, I summarize the context and aims of the research as well as the rationale, method, findings, and implications of each study. Second, I discuss implications of this research for the Theory of Planned Behaviour, both within and beyond the natural hazard context. Finally, I present potential applications of the key findings for interventions to increase preparation for natural hazards.

\section{Thesis Overview}

Context. The impacts of disasters, already affecting tens of millions of people and costing half a trillion dollars each year (Wallemacq \& House, 2018), are steadily rising as climate change increases the frequency and severity of weather-related events (Tippett, 2018) and population growth leads to more people exposed to natural hazards (Paton \& Buergelt, 2019). New Zealand is at risk from several types of natural hazards including earthquakes, tsunami, volcanic eruption, and high impact weather (Khan, Crozier, \& Kennedy, 2012). Even with these risks, however, most New Zealanders are not as prepared as they could or should be (Colmar-Brunton, 2018; Johnston et al., 2013), as is the case in several comparable international contexts (e.g., the United States: Bourque, 2013; Australia: Paton, 2019). Despite findings that every US\$1 of investment in resilience to such natural hazards has a return of up to US\$4 following an impactful event, most investment and research worldwide is focused on response and recovery, rather than reducing the risk from these hazards and increasing people's ability to cope with their impacts (Kellet \& Caravani, 2013).

Preparation for natural hazards takes many forms and can be conducted at many levels. At the national level, the New Zealand government has recently toughened legislation which requires the strengthening of earthquake-prone buildings (Building (Earthquake-prone Buildings) Amendment Bill, 2013). At the regional level, the Wellington City Council is investing millions of dollars to increase the ability of key infrastructure networks such as water and electricity to withstand the impacts of a large earthquake (Wellington City Council, 2017). Another important way to ensure individuals survive the initial impacts and reduce resulting disruption is to encourage preparation actions at the household level. Actions such as storing water and checking house foundations have long been recommended by organizations 
including New Zealand's National Emergency Management Agency, yet preparedness is increasing only slowly, if at all (Bourque, 2013; Johnston et al., 2013). Social science has examined the problem of why people do not prepare for such events for decades, both locally (Johnston et al., 2020), nationally (Becker, Paton, \& Johnston, 2015) and internationally (Lindell \& Perry, 2000; Solberg, Rossetto, \& Joffe, 2010). This thesis contributed novel information to the growing literature by identifying psychological constructs which influence the likelihood of people preparing for natural hazards and specific beliefs which lead to those constructs.

Aims. This thesis had two main aims. First, it aimed to identify the thoughts and beliefs which people hold about preparing for natural hazards that predict their behaviour. This information can then be used to design and improve public education campaigns with the purpose of encouraging preparation for natural hazards at the individual level. This aim included identifying new factors not established in the literature as well as identifying relevant beliefs at a more specific level than in most studies.

Second, this research tested statistical and methodological refinements to the central theoretical framework that was tested: the Theory of Planned Behaviour (TPB: Ajzen \& Madden, 1986). Since the development of this theory, several inconsistencies in how it is applied, measured, and analysed have emerged in the literature. This thesis addressed many of the more widely demonstrated and discussed inconsistencies, including the number of items used and the construction of measures (Armitage \& Conner, 2001), the inclusion of subfactors within the key predictors of attitudes (Darker, French, Longdon, Morris, \& Eves, 2007), norms (White et al., 2009) and behavioural control (Ajzen, 2002), the inclusion of indirect belief measures (Hardeman et al., 2002), and the compatibility of measures of intentions and behaviour (Ajzen, 2011a). As well as being one of the few applications of the TPB to natural hazard preparation and providing important insights for how the TPB is applied moving forward, the tests of this theory provide an empirical framework for evaluating the impacts of campaigns to increase preparation for natural hazards.

Attitudes. The first factor of the TPB theorized to explain behavioural intentions considers attitudes about the behaviour. Much past research in the natural hazard domain has explored attitudes about the risk when understanding motivations to prepare (e.g., risk perception); however, it is also important to consider attitudes about the behaviour of preparing, which is the intent of the attitudes component in the TPB (Lindell, Arlikatti, \& Prater, 2009). As with most of the TPB literature (Darker, French, Longdon, Morris, \& Eves, 2007), natural hazard research which does consider attitudes about behaviour tend to focus on instrumental 
attitudes (i.e., whether the behaviour will have the intended, positive outcome, also termed outcome expectancy and response-efficacy in the hazard literature; Becker et al., 2015; Bourque et al., 2012; Lindell \& Perry, 2000; Paton, 2019), neglecting experiential attitudes (i.e., whether the experience of carrying out the behaviour will be positive or negative). This focus on instrumental attitudes in previous research might reflect perceptions that these attitudes are more straightforward to shift than experiential attitudes. It is also possible that the existing bias towards exploring beliefs about the risk, rather than beliefs about the behaviour of preparing, has contributed to a focus on instrumental attitudes; while these do relate to perceptions of the behaviour, they pair logically with communications of potential impacts. That is, moving from communicating that a hazard might lead to certain outcomes to including information about how preparation will reduce those outcomes might be seen as a more natural progression than moving to include information about how preparing can be a positive experience.

Social norms. Social norms refer to guides around behaviour shared by a social group, which are not formalized as laws and are more context-dependent than traditions (Rimal \& Lapinski, 2015). There are two key dimensions to social norms; the first is the level at which they exist. Collective or actual norms refer to the objective existence of norms within a social group, whereas perceived norms refer to the perceptions that individual members of that social group have of the collective norms. These perceptions can be inaccurate, such that communicating collective norms are useful for changing behaviour in situations where collective norms are positive, whereas understanding perceived norms is useful for predicting behaviour. As such, this thesis focused on assessing perceptions of norms in line with TPB literature.

The second dimension of social norms to consider is the type of information to which they relate; descriptive norms refer to the prevalence of a behaviour within a social group, whereas injunctive norms refer to the approval of a behaviour (Rimal \& Lapinski, 2015). The original conceptualization of the TPB included subjective norms, which refer to general perceptions of pressure from other members of a social group to act in a certain way. Subjective norms have been implicitly and explicitly equated with injunctive norms for decades (e.g., Ajzen, 2002; White et al., 2009). The injunctive/descriptive distinction has been clearly advocated for in the norms literature generally (e.g., Cialdini, Reno, \& Kallgren, 1990; Park \& Smith, 2007; Vinnell et al., 2018); similar support for this distinction exists in the TPB literature (e.g., Ajzen, 2002; Ajzen, 2007), although is inconsistently advocated (e.g., Ajzen, 2013) and infrequently applied (e.g., Elliott \& Armitage, 2009; Farrow, Grolleau, \& Ibanez, 
2017; White et al., 2009). Given that descriptive and injunctive norms have been shown to target different motivational mechanisms (Hamann, Reese, Seewald, \& Loeschinger, 2015), the lack of use of this distinction has likely contributed to the weak and inconsistent findings for the norms component of the TPB.

Perceived behavioural control. The final factor theorized in the TPB to explain intentions is perceived behavioural control; as with attitudes and norms, this component has two proposed subcomponents, although the distinction here is the least developed of the twofactor distinctions within the theory. Firstly, self-efficacy refers to an individual's perceptions of their own capability to carry out a behaviour, considering factors such as difficulty, to form an opinion about whether they could engage in the behaviour. In contrast, controllability refers to an individual's perception of the behaviour as being volitional; that is, whether they can choose to engage, or not, in the behaviour. The distinction in the PBC component is the least clear of the TPB components; while experiential attitudes are not commonly studied they are conceptually clear from instrumental attitudes and the importance of distinguishing descriptive and injunctive norms is well supported in the literature. It is possible that inconsistent findings in the PBC component, and the lack of effects for controllability in this research, is due to conceptual overlap between controllability and self-efficacy. For example, if participants interpret questions on these measures as asking whether they "can" or are "able to" prepare, such an interpretation has both a permissive sense reflecting controllability (i.e., they are allowed to do it) and a capability sense reflecting self-efficacy (i.e., they are capable of doing it).

Study overview. The four empirical studies presented in this thesis include data from 2,298 participants, all recruited from the urban Wellington region (see Figure 1.1, page 21) and New Zealand (in Study 4). Study 1 comprised a preliminary test of the applicability of the theory to the specific context of natural disaster preparation. Study 2 built on the evidence from Study 1 that the theory is relevant to natural hazard preparation in New Zealand and tested all theorized components of the TPB (see Figure 7.1), including a proxy measure of subsequent preparation behaviour. Study 3 extended Study 2 by including a one-month follow-up to test whether intentions to prepare for natural hazard predicts preparation behaviour over time. Finally, Study 4 used a quasi-experimental design and partial TPB model (intentions and cognitions) to retrospectively evaluate the impacts of a previously-run common earthquake preparedness drill, ShakeOut, on cognitions related to preparation as well as knowledge and use of protective actions to take during shaking. 


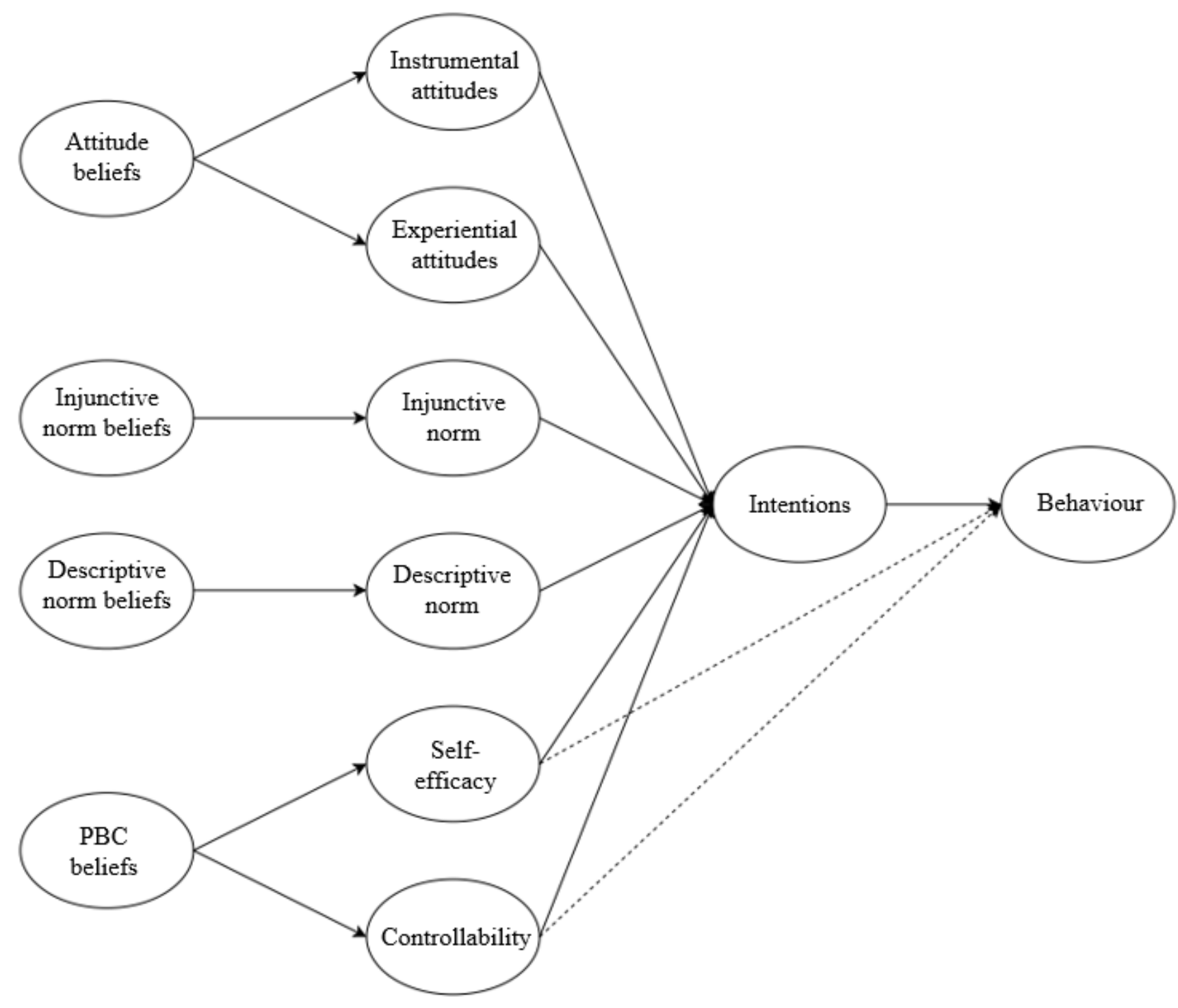

Figure 7.1. The Theory of Planned Behaviour with the cognition components divided into their theorized factors.

\section{Study 1 Summary and Implications}

Previous research demonstrates low rates of hazard preparation within Wellington (Johnston et al., 2013; Khan et al., 2012; McClure, Spittal, Fischer, \& Charleson, 2015), and recent public opinion surveys also report low preparedness across New Zealand (ColmarBrunton, 2018). The first objective of Study 1 was to confirm that preparation levels are still low enough to warrant efforts to increase them (McKenzie-Mohr, 2011), as well as to 
reproduce McClure et al.'s (2015) findings that the main barrier for most preparation actions is not thinking about it, followed by not seeing it as a priority. Finally, given the limited number of applications of the TPB to natural hazards (Najafi, Ardalan, Akbarisari, Noorbala, \& Elmi., 2017), this study used established TPB measures (Ajzen \& Madden, 1986) to demonstrate that this theoretical model can identify significant associations between cognitions and intentions in the context of Wellington residents' natural disaster preparation (this target behaviour was rephrased as "natural hazard" after Study 2).

Of the 18 preparation actions listed, participants had performed on average only five of them with a single action (storing water) performed by a majority of participants (54.8\%). Also consistent with previous research, participants had performed more survival actions (i.e., actions to help them survive after an event such as storing food) than mitigation actions (i.e., actions to reduce damage and injury during an event, such as securing tall furniture; Russell, Goltz, \& Bourque, 1995). They also gave either "hadn't thought about it" or "haven't got around to it" as the main reason for not completing most actions. Descriptive norms and perceived behavioural control were significantly and positively associated with past preparation, and attitudes were significantly associated with intentions to prepare in the future. Descriptive norms have been found to lower the likelihood of people preparing in New Zealand contexts (Becker, Paton, Johnston, \& Ronan, 2014); the positive association here might reflect a perceptual bias in that those who have prepared are more likely to think that others have also prepared. However, because this study is correlational, the direction of any effects cannot be determined.

The self-efficacy component of PBC has long been shown to positively relate to preparation (e.g., Levac, Toal-Sullivan, \& O’Sullivan, 2012; Lindell \& Perry, 2000). Some evidence demonstrates that people who feel that they have more control over their future have prepared more for earthquakes (Rustemli \& Karanci, 1999), but the effect of perceived control over preparing for natural hazards specifically has not been established. Similarly, the positive association found in this research between attitudes about preparing and intentions to prepare aligns with much previous research showing the importance of outcome expectancy and fatalism (similar to instrumental attitudes) to preparation (Becker et al., 2015; Bourque et al., 2012; Lindell \& Perry, 2000; Paton, 2019). As with the association between perceived behavioural control and preparation, this association between attitudes and intentions to prepare supplied the basis for Study 2 to further explore the role of the subfactors (self-efficacy and controllability; instrumental and experiential attitudes). 
The finding that preparation is low overall and that participants reported that cognitive barriers (i.e., more than practical barriers such as cost) are inhibiting behaviour supported further investigation into beliefs around preparing. It is important to note that this study tested self-reported perceived barriers and therefore assumes accurate self-awareness of participants. It is likely that the barriers examined in Study 1 are not discrete and that cost, for example, does play a role in inhibiting more preparation actions than identified. Low levels of preparation provide room for meaningful increases in behaviour (McKenzie-Mohr, 2011), while the clarification of barriers which are related to personal beliefs (rather than barriers which are affected to a lesser extent by beliefs, such as cost; Ajzen, 2011a) provided a basis for Studies 2 and 3 to identify those most impactful beliefs. Further, the significant associations of all tested TPB factors (excepting injunctive norms) with either past preparation or intentions to prepare supported the goal of testing a more thorough implementation of the model.

Study-specific limitations. As Study 1 used traditional TPB measures, the methodology did not address the common inconsistencies found in much of the literature described earlier. Because this study represented a preliminary test of applicability, rather than a test of the theory from which to draw strong inferences, this limitation is not problematic for the specific contributions of this study. However, this issue did clearly require addressing in the subsequent studies. Moreover, the Study 1 sample included a disproportionate number of female participants. This sampling limitation also occurred in Studies 2 and 4, so it will be discussed in more detail in the following Limitations section which discusses limitations common across all four studies.

\section{Study 2 Summary and Implications}

Study 2 built on Study 1 by testing intentions to prepare for natural hazards in the full TPB model; this included both the two-level distinction between direct and indirect measures by measuring belief constructs and the two-factor distinction by including the distinctions between instrumental and experiential attitudes, injunctive and descriptive norms, and selfefficacy and controllability. Further, to test for preliminary evidence that intentions to prepare relate to behaviour, Study 2 included an objective behaviour measure at the end of the survey. The measures in this study incorporated recommendations from three TPB questionnaire construction guides (Ajzen, 2002; Ajzen, 2013; Francis et al., 2004), so that all factors were measured with four items, with minimal changes to the wording to fit the current behavioural context.

All belief factors were significantly associated with the related cognitions, while most of those cognitions were associated with intentions to prepare for either natural hazards or 
natural disasters. The positive associations of instrumental attitudes and self-efficacy with intentions replicate previous findings, as explained above in the Study 1 summary. However, Study 2 also adds to the knowledge of both Study 1 and previous evidence by demonstrating the importance of experiential attitudes and perceived injunctive norms to intentions to prepare. Injunctive norms have been shown previously to increase support for earthquake-related legislation (Vinnell, Milfont, \& McClure, 2018) but have not been clearly shown to relate to intentions to prepare. Further, few, if any, studies have demonstrated that more positive experiential attitudes relate to stronger intentions to prepare. Both findings in the present research offer new and useful insights to consider when understanding and increasing preparation behaviour.

Descriptive norms in Study 2 did not demonstrate a significant association with intentions to prepare; this contrasts with previous research which suggests a negative relationship between descriptive norms and preparation (Becker, Paton, Johnston, \& Ronan, 2014) and other studies which have demonstrated positive effects on preparation (Bourque, 2013; Ozaki \& Nakayachi, 2015). The mean score for descriptive norms $(M=3.81)$ did differ statistically from the scale midpoint (4), though the difference is small ( $p<.05, d=0.13)$. It is possible that the direction of the relationship between descriptive norms and preparation depends on whether people perceive a minority norm (i.e., most other people are not preparing; Becker et al., 2014) or a majority norm (Ozaki \& Nakayachi, 2015). The non-significant association here could be because the norm perceived by the participants of the study is relatively neutral; neither held by a small enough minority to have a negative effect nor a large enough majority to have a positive effect. This finding therefore demonstrates the importance of understanding the research context when deciding if or how to explore the role of social norms in hazard preparation. In social groups where preparing is common, this can be emphasized to increase behaviour. In social groups where preparing is not common, interventions can aim to shift the norm upwards to a point where it has a positive impact. One possible way to do so is presented later in this discussion.

Second, Study 2 included an experimental manipulation. There is a developing argument within the research in this domain to stop classifying disasters as "natural" (e.g., Chmutina \& von Meding, 2019). Despite evidence from other domains such as climate change that small alterations in wording can have significant impacts on intentions to act (e.g., climate change versus global warming: Benjamin, Por, \& Budescu, 2017; war versus race against climate change: Flusberg, Matlock, \& Thibodeau, 2017), few studies have examined the suggested benefit of dropping "natural" so that people attribute the impacts of natural hazard 
events to human causes (e.g., McClure, Sutton, \& Sibley, 2007), or using the term natural hazard rather than natural disaster. Study 2 tested whether thoughts, beliefs, intentions, and behaviour related to preparation differed depending on whether participants were asked to consider "natural disasters" or "natural hazards". A randomly assigned half of the participants received a survey which exclusively referred to "natural disasters" with the other half receiving a version that was identical except for the substitution of the term "natural hazard".

The results showed that the TPB model in the "natural hazard" group differed meaningfully from the model in the "natural disaster" group. Experiential attitudes, injunctive norms, and self-efficacy were positively associated, and controllability negatively associated with intentions to prepare for natural disasters, explaining approximately $41 \%$ of the variance. In contrast, instrumental attitudes, experiential attitudes, and self-efficacy were positively associated with intentions to prepare for natural hazards, explaining approximately $47 \%$ of the variance. Furthermore, intentions to prepare for natural hazards was significantly and positively associated with behaviour (requesting an earthquake-planning guide), whereas intentions to prepare for natural disasters was not. More research is needed to fully understand the impacts of variations in the terms used when communicating with the public. However, based on the greater variance explained in intentions to prepare and the significant association with behaviour in the "natural hazard" group compared to the "natural disaster" group, as well as the logic of the argument against using the latter term (Chmutina \& von Meding, 2019; Kelman, 2018), these findings from Study 2 provide important preliminary support both for further empirical exploration as well as the shift away from the term "natural disaster". They also informed the decision to use "natural hazard" in the subsequent study.

Study-specific limitations. Study 2 had two main limitations, beyond the demographic bias mentioned above and discussed later. First, several scales demonstrated average to poor reliability, in particular attitude beliefs. While the reliability of these scales was acceptable, there is a need for more rigor at the belief-elicitation stage. However, this step is often skipped entirely by researchers developing TPB measures (Darker et al., 2007) so that the small-sample $(N=15)$ method used here is still an improvement on much of the literature.

Second, although many TPB studies do not include a measure of behaviour at all, the concurrent behaviour measure used in this study does not allow for inferences as strong as the behavioural data gathered after the initial TPB assessment (Armitage, Reid, \& Spencer, 2013). The use of information seeking as a preparation behaviour has mixed support within the literature. Kahlor, Olson, Markman, and Wang (2018) suggest that information seeking is not a good proxy for behaviour, while receiving information about a natural hazard risk has been 
shown to lower concern about that risk (Ballantyne, Paton, Johnston, Kozuch, \& Daly, 2000). However, the information offered to participants in this research focuses on actions to mitigate the well-known risk of earthquakes, which likely would not lead to a reduction in concern like that found by Ballantyne et al. (2000). Although Paton, Smith, and Johnston (2005) demonstrated that intentions to seek information did not predict preparation action, risk information has been shown to positively influence preparedness (Bourque, 2013; Perry \& Lindell, 2008) and information-seeking has been treated as a preparation action (Paton, 2019).

Paton et al. (2005) possibly did not find that intentions to seek information predicted preparation because they asked about four well-known actions (securing cabinet doors, securing tall furniture and heavy items, making an emergency plan, and making an emergency kit) for which participants likely did not need more information. It is logical that those participants who did request the planning guide will benefit from better knowledge of actions they can take to prepare themselves, given that participants often responded that they had not thought about undertaking a particular action in Study 1. This finding also suggests that intentions to carry out different types of preparedness, in this case seeking information compared to mitigation and survival actions, reflect differences in the beliefs that lead people to engage in those various types of behaviours.

Further, Paton et al. (2005) tested whether intentions to carry out one specific action, information-seeking, predicted four different specific preparation actions, none of which were seeking information. The lack of association between intentions to seek information and the specific preparation actions could therefore also be due to incompatibility between the measures which can lead to non-significant effects (Ajzen, 2011a). Study 3 used intentions to prepare generally to predict one specific behaviour; this is likely better than using intentions to undertake one specific type of preparation to predict different specific behaviours (Darker et al., 2007). However, the limitation of the behaviour measure used in Study 2, as well as the low reliability of attitude beliefs and distinct gender bias, were addressed in Study 3.

\section{Study 3 Summary and Implications}

Study 3 built on the previous studies in three main ways. First, this study used random representative sampling with the aim of recruiting participants with less demographic bias and who better reflect the urban Wellington population than Studies 1 and 2. While the gender ratio in the Study 3 sample did closely align with the population ratio, participants were both older than the population median and most minority ethnicities were less represented than in Studies 1 and 2. The weight of past evidence suggests that these demographic variables affect risk perception (Lindell \& Perry, 2000) but have limited effects on preparation (e.g., Becker et al., 
2015; Bourque, 2013 Solberg et al., 2010). Therefore, while a fully representative sample would have been preferred, any demographic biases in this research are likely to have negligible impacts on the overall results.

Study 3 also included a one-month follow-up survey which measured preparation behaviour using retrospective self-reports to test how well intentions to prepare predict preparation. While the recruitment method resulted in a smaller sample size than anticipatedso that the planned analysis, including beliefs, cognitions, intentions, and behaviour in a single model, could not be run - intentions predicted both participants' single-item self-report of their preparation over the month between the two study time points as well as the number of specific actions they reported taking, consistent with expectations based on most TPB research (e.g., Hardeman et al., 2002) and hazard-related research (e.g., Paton et al., 2005). In contrast with Study 2, only self-efficacy predicted whether participants would request the earthquakeplanning guide, with the odds of this behaviour decreasing with higher self-efficacy. This difference in the ability of intentions to predict preparation compared to information seeking matches previous findings that the two types of intentions are formed differently; for example, self-efficacy has been found to associate with intentions to seek information (although in that study the relationship was positive) but not with intentions to prepare (Paton et al., 2005).

Finally, the attitude belief measure was extended to allow for a testing of the instrumental and experiential distinction at this level. While this allowed refinements to the measures so that both were within acceptable ranges of reliability, this distinction did not increase the strength of the association between the attitude beliefs and the related attitude cognition. However, both self-efficacy beliefs and controllability beliefs demonstrated stronger associations with the related cognitions than did general PBC beliefs. These findings support further exploration of the importance of this two-factor distinction at the belief level.

As well as fulfilling one aim of this thesis (i.e., the development and testing of a TPB questionnaire), Study 3 also fulfils the second primary aim of identifying cognitions and specific beliefs about natural hazard preparation which relate to subsequent behaviour and can be targeted in interventions. Consistent with Study 2, experiential attitudes, instrumental attitudes, and self-efficacy were significantly and positively associated with intentions to prepare. While previous research applying the TPB to natural hazards has demonstrated positive associations of attitudes, norms, and $\mathrm{PBC}$ with intentions to prepare (Bates, Quick, \& Kloss, 2009; Morrison, Lawrence, \& Oehmen, 2014; Najafi et al., 2017), these studies did not apply the two-factor or two-level distinction as were used here. The test of more specific factors 
in this research therefore represents an improvement on those previous studies and offers more useable understanding.

There is little to no previous evidence demonstrating the importance of experiential attitudes or the beliefs that preparing can be fun and rewarding for natural hazard preparation, so these findings in particular offer considerable value. Much previous research has shown that self-efficacy and variables similar to instrumental attitudes (i.e., outcome expectancy, response-efficacy, and fatalism; Becker et al., 2015) are important for understanding why people do and do not prepare for natural hazards (e.g., Lindell \& Whitney, 2000; Paton et al., 2005; Solberg et al., 2010). However, by including belief measures, Study 3 was able to demonstrate specific contributions to the development of instrumental attitudes and selfefficacy.

The significant belief that preparing is a life-saving action is consistent with the finding by Najafi, Khankeh, Elmi, and Pourvakhshoori (2018), though Study 3 adds to this finding by providing statistical evidence that this belief is related to behaviour via instrumental attitudes and intentions, although these relationships were tested separately rather than in a single model due to lack of power. Further, the identification of the important beliefs that preparing will help people "get through a natural hazard event better" and that they can make the effort to prepare represent novel findings in this area. As far as I am aware, no study has both identified such specific relevant beliefs and statistically demonstrated their association with behaviour. Therefore, Study 3 presents a number of findings which replicate previous research as well as findings which offer important, unique contributions to our understanding of why people prepare for natural hazards.

Study-specific limitations. The main limitation specific to Study 3 is the small sample size. While the Time 1 sample $(N=151)$ included enough data for structural equation modelling, the lower power at Time $2(N=63)$ did not allow for testing of the full TPB model simultaneously as planned. Splitting the analyses into explaining intentions and predicting behaviour separately weakens the confidence in the inferences drawn from the two separate explanatory and predictive models. However, given that the findings align with the findings of Study 2, these inferences are still strong enough to be informative for researchers and practitioners undertaking behaviour change interventions.

\section{Study 4 Summary and Implications}

One of the primary aims of this thesis was to identify beliefs about natural hazard preparation which can be used to design public campaigns. However, these beliefs can also be applied to evaluate and adapt an existing campaign, which is more efficient than creating an 
entirely new one (Joffe, Perez-Fuentes, Potts, \& Rossetto, 2016). Further, the timeframe of this thesis was not sufficient to design, run, and evaluate a behaviour change intervention informed by Studies 1, 2, and 3. However, an existing programme of evaluation for the New Zealand ShakeOut drill allowed the opportunity to test whether the TPB measures developed in this thesis are useful for evaluating interventions.

Study 4 employed a quasi-experimental design to evaluate the ShakeOut earthquake drill, developed in the United States and run nationally in New Zealand in 2012, 2015, 2018, and 2019, as a case study. As well as testing whether participating in the ShakeOut drill achieves its primary aim of learning the protective actions "drop, cover, and hold", Study 4 evaluated the impact of drill participation on variables of the TPB (instrumental and experiential attitudes, injunctive and descriptive norms, self-efficacy and controllability, and intentions to prepare). Consistent with an evaluation of the 2012 and 2015 ShakeOut drill (Vinnell, Wallis, Becker, \& Johnston, 2020), the results of Study 4 show that those who participated in the 2018 drill had better knowledge of what to do during shaking and use of those actions during earthquakes than those who did not participate. While there is room for improvement in these outcomes, the ShakeOut drill appears effective at achieving its main aim.

Previous research has demonstrated that participating in the ShakeOut drill increases knowledge of protective actions (Johnson et al., 2014). However, it is unwise to assume that knowledge is sufficient for these actions to be employed; one study found that children often did not know how to apply "drop, cover, and hold" in situations other than the one in which they practised (Johnson et al., 2014). Study 4 contributes new evidence that those who have participated in the drill are also more likely to undertake those protective actions in actual earthquake shaking. Given the high proportion of injuries during earthquake shaking which can be prevented with these actions (Johnston et al., 2014), the results of Study 4 support the continued application of the ShakeOut drill and suggest ways that the drill can be improved.

Further, those who participated in the drill had higher critical awareness, were more likely to have sought additional information on earthquake preparation, demonstrated less fatalism bias, and showed notably higher scores on intentions to prepare and five of the TPB cognition components than those who did not participate (only controllability was no higher in the Treatment group than the Control group). Past evaluations have demonstrated benefits of the ShakeOut drill for earthquake preparedness (Becker, 2009; Orchiston, Manuel, Coomer, Becker, \& Johnston, 2013; Tipler, Tarrant, Johnston, \& Tuffin, 2016), outcome expectancy (similar to instrumental attitudes), and self-efficacy (Adams, Karlin, Blakely, \& Glik 2017). However, Study 4 presents a more thorough evaluation than any of the evaluations conducted 
previously, replicating findings of those evaluations as well demonstrating novel positive effects of participation on critical awareness, fatalism, experiential attitudes, and descriptive and injunctive norms. These novel findings show that the ShakeOut drill impacts a range of judgments and behaviours relating to earthquake preparedness. It is logical, therefore, to continue implementing this drill but to adapt the messaging involved to target the beliefs identified in Study 3.

Studies 1 to 3 used the TPB to identify key factors relevant to preparation for natural hazards which can be targeted in education campaigns, in line with recommendations for empirically-based interventions (e.g., Levac et al., 2012; Ronan et al., 2008). Study 4 demonstrated that the TPB can also be used effectively to evaluate such interventions. The TPB is most commonly used in this latter sense (i.e., to evaluate rather than inform interventions) in other domains such as health behaviour (Elliot \& Armitage, 2009; Hardeman et al., 2002). The support in this thesis for the relevance of most TPB components to natural hazard preparation, as well as the evidence in Study 4 for impacts of an existing intervention on most components of the theory, identifies the TPB as a valuable evaluation tool for hazard researchers and practitioners.

Study-specific limitations. Study 4 had two limitations distinct from the other studies. First, the long delay between participating in the drill and the evaluation likely lowered the accuracy of reporting of behaviours undertaken at the time of the drill. However, most of the factors of interest involved judgments either at the time of the evaluation or required participants to report behaviour undertaken during the interim. The former responses cannot logically be impacted by the time delay while there is little reason to expect a meaningful impact on the latter responses. Further, this time delay was necessary to ensure that a sufficient number of participants $(n=213)$ had felt an earthquake in which to use the "drop, cover, and hold" response. One of the main contributions of this study is the finding that knowledge of the recommended protective actions largely translates to use of those actions, which helps to outweigh the potential confounds introduced by the long delay. Among those who participated in the 2018 New Zealand ShakeOut drill, the percentage of people who knew what actions to use $(64 \%)$ was similar to the percentage of people who have used "drop, cover, and hold" during all $(51 \%)$ or some $(22 \%)$ of the earthquakes they have felt. This similarity provides preliminary but crucial evidence for the assumption that people with correct knowledge are more likely to actually apply that knowledge.

Second, this interpretation assumes that the differences between the Treatment and Control groups are the result of impacts of participating in the drill. It is also possible that 
differences in the type of people who do and do not participate in the drill contributed to the group effects. However, participants in the two groups did not differ significantly on any of the demographic variables measured. Even accepting the assumption that pre-existing differences are the main cause of the group effects, the findings still provide ways in which the campaign messaging can be tailored to increase the likelihood of people participating. For example, those who participated in the ShakeOut drill reported more positive instrumental attitudes than those who did not. If we assume that those people participated because they have more positive attitudes than those who did not participate, then messaging to encourage people to sign up can emphasize that practising "drop, cover, and hold" helps people to undertake the actions during actual shaking (as found in Study 4) and therefore can help prevent them from being injured during earthquakes. Given that instrumental attitudes were significantly related to intentions to prepare among the Treatment group, such messaging is likely to be beneficial even for those who participate in the drill.

\section{Limitations of the Research Across the Studies}

Beyond the study specific limitations discussed above, the present research has other important limitations across all studies. In particular, there are limitations regarding sample demographics, generalizability, and reliance on self-report measures. Explanations and considerations of the impacts of these limitations are presented below.

Sample demographics. Across all studies, one consistent limitation is the lack of representativeness in the samples. Study 3 used random sampling from the electoral roll to address the gender bias in the previous two studies. While the ratio of men to women in Study 3 was virtually identical to the ratio in the Wellington region, participants were on average older than the median age, and all but one minority group were less represented than in the other studies. However, the significant cognitions identified in Study 3, with an ideal gender balance but poorer age and ethnicity representation, were the same as those identified in Study 2 , with a worse gender balance but better age and ethnicity representation. While it would be preferable to identify these thoughts and beliefs with a single representative sample, the similarity of results across Studies 2 and 3 suggests that these thoughts and beliefs are consistent across different demographic groups. The balance of previous research indicates the overall lack of influence of demographics such as age, gender, and ethnicity on natural hazard preparation (Becker et al., 2015; Bourque, 2013; Lindell \& Perry, 2000). As well as implying that the beliefs identified in Study 3 are relevant for urban Wellington, the negligible role of demographic factors suggests that the findings here may apply to other populations nationally and internationally. 
Generalizability. Despite the limited influence of demographic factors, though, it is important to consider specific differences when applying research findings in different contexts. The most salient natural hazard in Wellington is that of earthquakes (e.g., Khan et al., 2012). As such, the preparation actions reported in this thesis are mostly geared towards earthquake preparedness with some, but not full, cross-hazard application. It is possible that people in other areas of New Zealand (or the world) with a different salient natural hazard hold different beliefs about the preparation actions recommended to deal with that hazard. For example, people living in Auckland, New Zealand's largest city, may view their most salient natural hazard risk of volcanic eruptions differently than how residents of Wellington view earthquake risk and preparation. Volcanoes are also sudden onset, in comparison to hazards such as sea-level rise, but can give enough warning to evacuate before any major impacts. Further, notable eruption events in New Zealand are relatively few (particularly compared to earthquakes) and those which exist in living memory (e.g., 2019 Whakaari/White Island, 1995 and 1953 Ruapehu events) were not located in Auckland, whereas Wellington has recently experienced impacts from earthquakes (e.g., 2016 Kaikōura and 2013 Cook Strait events). Work using the Community Engagement Theory has demonstrated that intentions to prepare and outcome expectancy have substantially similar associations with preparedness for tsunami, earthquake, wildfire, and pandemic hazards (Paton, 2013), suggesting that the pattern of findings in this research could be expected in other hazard contexts.

Similarly, people are more likely to engage in preparation for earthquakes if that action has other benefits or applications, such as an emergency torch also being useful for power cuts (Lindell, Arlikatti, \& Prater, 2009). Whether a recommended preparation action, for whatever hazard, has other benefits may therefore influence uptake of that action. Both the rates of preparation and influences on intentions to prepare found here, for mostly earthquake-related actions, may therefore differ depending on the hazard context.

Further, not all findings may generalize to international contexts. Communities whose governments have less legislation and invest less in hazard resilience may have different beliefs regarding preparing; for example, developing countries typically suffer more than six times as many deaths per disaster compared to high-income countries and are seeing a more rapid increase in the frequency of building collapse (Lindell, 2013).

Further, evidence demonstrates that TPB variables may differ across cultures (e.g., Fischer, Karl, \& Fischer, 2019; Walker, Courneya, \& Deng, 2006) as well as differences in how risk is perceived and acted upon (Glik, Eisenman, Zhou, Tseng, \& Asch, 2014). Researchers and practitioners intending to apply the findings of this research would do well to 
consider the level of overlap between the context under study here and the context in which the findings are applied. Further, interventions should pilot test for effectiveness, regardless of empirical support for the relevance of targeted beliefs (McKenzie-Mohr, 2011). Ostensibly, these pilot tests serve to support the behaviour change mechanism used in the intervention, but they can also be used to ensure that the beliefs chosen to target are indeed relevant for the particular population.

Self-report measures. Finally, this research uses self-report measures (although it does also use the behaviour measure of requesting an earthquake-planning guide). This is common in many areas of social psychology including TPB research (Hardeman et al., 2002) as selfreports are more easily obtained than objective measures (Ajzen, 2002). However, the research here does not require participants to recall any information from more than a month ago (excluding some questions in Study 4) so memory is unlikely to be an issue. The main relevant limitation of self-report used in this research therefore is the potential response bias. Even in confidential surveys, people produce socially desirable responses where they give the answer which they feel would be seen as "correct" by their peers (Fisher, 1993; van de Mortel, 2008). Measures to control for social desirability bias in survey responding do exist but including these would have added more questions to already long surveys, and the impact of social desirability responding may be weaker than is often assumed (Milfont, 2009). Further, although the accuracy of self-report measures varies between behavioural contexts (Ajzen \& Fishbein, 2004), such measures can be acceptably reliable, especially if they use more than a single item as was the case in this research (Ajzen, 2002).

Quantitative methods. Historically, psychological research has been dominated by quantitative methods (Gelo, Braakman, \& Benetka (2008). There are benefits to quantitative methods; surveys are quicker and less resource intensive than common qualitative methods such as interviews and focus groups, while the ability to use measures consistently across studies makes results more easily comparable. Quantitative methods are suited for theory testing, examining predicted relationships rather than forming a deep understanding of phenomena, which is more aligned with the aims of this thesis. Larger samples which better represent populations than qualitative methods (Sale, Lohfeld, \& Brazil, (2002) also aligns better with the aims of this thesis to inform broad campaigns, rather than targeting communities or specific sections of the population. Because researcher and participant are independent, quantitative methods allow for the study of factors without influencing them; however, quantitative methods lessen the potential of meaningful interpretation, whereas qualitative methods provide richer detail of the properties of a variable (Gelo et al., 2008). Qualitative 
methods are therefore valuable for gaining a deeper understanding of behaviour but due to the more intensive methods required, they tend to produce findings which are less generalizable. Mixed methods approaches are increasingly being used to leverage the benefits and address the limitations of both qualitative and quantitative methods (Gelo et al., 2008). However, the two methods cannot be combined for cross-validation (Sale et al., 2002). Rather, "good" mixed methods research requires a thorough understanding of the fundamental differences in approach and philosophies between quantitative and qualitative (Landrum \& Garza, 2015). Quantitative methods were used in this thesis as they were sufficient to address the aims of the research. However, qualitative and mixed methods approaches offer several possibilities for future research to expand the findings of this thesis. Such potential avenues are presented in the following sections, in particular those discussing framing effects, barriers to preparing, and social norms.

\section{Theoretical Implications}

The Theory of Planned Behaviour has been used extensively over the decades since its creation to explain and predict a wide range of behaviours (for meta-analytical reviews, see Armitage \& Conner, 2001; Guo, Wang, Liao, \& Huang, 2016; McDermott et al., 2015; McEachan et al., 2011; Riebl et al., 2015; Rivis, Sheeran, \& Armitage, 2009; Topa \& Moriano, 2010; Tyson, Covey, \& Rosenthal, 2014). Over this time, several extensions and refinements to the theory have been proposed but inconsistently applied. One of the two primary aims of this thesis was to test the full TPB model to make recommendations about whether and how these refinements should be applied. Unique theoretical contributions of each study have been briefly discussed in the above specific summary sections. The following sections summarize these contributions and make recommendations for future TPB research and applications.

The two-factor distinction. The first extension introduced the "two-factor" distinction for all TPB components (Conner, Hugh-Jones, \& Berg, 2011). This extension separated the attitudes component into instrumental and experiential attitudes, the norms component into injunctive and descriptive norms, and the perceived behavioural control component into controllability and self-efficacy (Ajzen, 2002). Few studies have applied this two-factor distinction to all of these cognition components (Conner et al., 2011), and within these studies there are inconsistencies as to which two factors are included. For example, Conner et al. (2011) split attitudes into instrumental and affective whereas Ajzen (2006b) suggests splitting attitudes into instrumental and experiential. Overall, the results presented in this thesis support extending the single factor constructs to include subfactors as this increases the specificity and therefore the usefulness of identified predictors. 
In Studies 2 and 3, both instrumental attitudes and experiential attitudes uniquely explained variation in intentions to prepare. These findings contribute important understanding of which types of attitudes are important for natural hazard preparation. Previous TPB research typically defaults to only using instrumental attitudes (Darker et al., 2007) so that the importance of experiential attitudes to other behaviours may not have been established because it has not been tested, as was the case for natural hazard preparation. Previous research on attitudes towards preparation actions (as opposed to attitudes towards hazards) tends to focus on the outcomes of those actions (i.e., instrumental attitudes; Lindell et al., 2009). Hence, including the two-factor distinction for attitudes in the present research enabled it to identify experiential attitudes as relevant to natural hazard preparation, as well as to replicate previous findings about the importance of instrumental attitudes to (Becker et al., 2015; Bourque et al., 2012; Lindell \& Perry, 2000; Paton et al., 2010; Terpstra \& Lindell, 2012). This contribution would not have been possible without deliberately testing for both types of attitudes.

The distinction between injunctive and descriptive norms is well established in the norms literature (Cialdini, Reno, \& Kallgren, 1990; Park \& Smith, 2007), including the area of natural hazards (Vinnell et al., 2018). The findings in this thesis support the continued application of this distinction, both when considering preparation for natural hazards and when applying the TPB to other behaviours, as there were meaningful inconsistencies in whether each of the two paths were statistically significant across the models. Overall, the results show that injunctive norms are slightly more reliably related to intentions to prepare for natural hazards than descriptive norms but neither demonstrated conclusive relationships. As with attitudes, these variations in the importance of descriptive versus injunctive norms would not have been identified had the original factor of subjective norms been tested.

Finally, the distinction between the two PBC factors is shown to be valuable. As expected, self-efficacy demonstrated significant, positive associations with intentions to prepare (Becker et al., 2015; Lindell \& Whitney, 2000; Solberg et al., 2010) as well as predicting information seeking in Studies 2 and 3 (although this association was negative, which was unexpected but explainable). Consistent with Ajzen's (2011b) suggestion that both self-efficacy and controllability may be relevant only for some behaviours, the implication of this research that overall controllability has a minimal role in intentions to prepare for natural hazards would not have been identified without making the subcomponent distinction. Prior to this thesis, little evidence from previous research showed whether controllability played a role in preparation for natural hazards. If this non-significant role of controllability, explicitly distinct from self-efficacy, is demonstrated in subsequent research, it may be advisable to drop 
controllability and examine only self-efficacy. Without supporting evidence, however, including the two-factor distinction for PBC is recommended, as doing so provides more specific and therefore applicable information for efforts to change behaviour.

Beyond systematically applying the two-factor distinction for the key TPB constructs assessing cognitions, the research in this thesis also advanced previous research by examining possible distinctions at the belief level. The clearly established distinction between injunctive and descriptive norms, while still not consistently applied in the TPB literature, has led to the development of specific measures for descriptive and injunctive norm beliefs. Study 3 extended the two-factor distinction to the other beliefs, modelling separate experiential and instrumental attitude beliefs as well as separate self-efficacy and controllability beliefs. Although the model fit did not improve, the separate PBC beliefs were more strongly associated with their relevant cognitions than were general PBC beliefs. The same was not found for the separate attitude beliefs; the decrease in the number of items and weaknesses in belief-elicitation potentially contributed to this lack of improvement. The stronger paths when PBC beliefs were distinguished support the extension of the two-factor distinction to the belief level as well. As far as I am aware, no previous study has differentiated the two factors of attitudes and PBC at the belief level in this way. Ajzen recommends using the TPB to identify beliefs to target in an intervention (Ajzen, 2011a; Hardeman et al., 2002), as changing beliefs is likely to be more effective than trying to change cognitions (Darker et al., 2007; McMillan et al., 2009). Given this proposal, evidence that these constructs can be made more specific, and therefore informative, by testing subfactors improves the ability of the TPB to inform effective interventions.

As well as informing interventions, Study 4 tested the utility of the TPB to evaluate the ShakeOut earthquake drill, an intervention to increase preparedness for earthquakes. Although previous studies have used the TPB as a framework to evaluate behaviour change interventions (Hardeman et al., 2002; Tyson et al., 2014), few of those studies have considered the two-factor distinction or natural hazard preparation. Study 4 demonstrates that the distinction between instrumental and experiential attitudes, injunctive and descriptive norms, and self-efficacy and controllability increases the usefulness of information attained in such evaluations. It also shows that the TPB can be used to evaluate the impacts of interventions to encourage preparation for natural hazards.

In summary, the present research supports the two-factor TPB model, with attitudes divided into instrumental and experiential, norms into injunctive and descriptive, and PBC into controllability and self-efficacy. To date, studies applying the theory have not applied these 
distinctions consistently, which likely contributes to the conflicting effects found in metaanalyses (e.g., Armitage \& Conner, 2001). Research which has aimed to follow the two-factor TPB includes different distinctions than recommended by other authors (e.g., adding affective rather than experiential attitudes: Ajzen, 2006b; Conner et al., 2011). The current confusion in the field is understandable given that the different behaviours to which the theory can be applied limits the possibility of having a single uniform questionnaire. Further, the way in which the theory's creator explains the constructs differ. In a 2002 questionnaire construction guide, Ajzen presents all three two-factor distinctions applied in this study. However, these distinctions are not mentioned in a 2011 book chapter or included in a 2013 example questionnaire (Ajzen, 2002; Ajzen, 2011a; Ajzen, 2013). The inconsistent inclusion of belief items between studies could also result from changing recommendations. In 2002, Ajzen stated that beliefs are indirect measures of the same latent construct as the direct cognition measures, whereas in 2011 he stated that beliefs lead to and predict those cognition measures (Ajzen, 2002; Ajzen, 2011a).

This thesis adds substantial support to the existing evidence in favour of the argument within the TPB literature that the cognition components should be treated as including two factors. Further, the findings show that those factors should be instrumental and experiential attitudes, injunctive and descriptive norms, and self-efficacy and controllability. The evidence here for extending this two-factor distinction to the belief level is preliminary but sufficient to recommend further exploration, particularly given the importance of beliefs in behaviour change efforts.

Measurement and modelling. Several inconsistencies in how the TPB is measured and modelled have been suggested as explanations for inconsistent results within the literature (Armitage \& Conner, 2001). As well as not including the belief constructs (Hardeman et al., 2002), studies often create their own measurement items rather than following a tested and widely used scale. Such a scale is difficult to create given that items need to be adapted for each behavioural context. However, a set of stem items that can be easily adapted would help to reduce issues in measurement. This thesis used items mainly from three questionnaire development guides (Ajzen, 2002; Ajzen, 2013; Francis et al., 2004). I do not suggest that items ought to be drawn from several sources; rather, several guides were necessary to obtain items for all constructs as no single guide included both beliefs and all two-factor distinctions. The items developed in this thesis therefore represent a rare, if not unique, example of a questionnaire measuring all proposed constructs with a set of tested items that could be easily 
adapted to other research domains. Such a questionnaire should help to reduce the inconsistencies within TPB research.

Further, many studies use regression analysis to model the TPB (Topa \& Moriano, 2010), while others use structural equation modelling. This latter analysis is recommended by Ajzen (2011a) as it allows for all paths to be tested simultaneously and deals better with error and variances in the data (see also Topa \& Moriano, 2010). While the small sample size in Study 3 did not allow for all paths to be tested in a single model, the continued use of structural equation modelling is recommended where the methods available to researchers allow for the recruitment of enough participants. These methods are not necessarily restricted by resources. Study 2 and Study 4 both required far less time and money than did Study 3 but returned much larger samples.

Finally, not all TPB studies test for a direct effect of PBC on behaviour, and those which do demonstrate inconsistent findings (Armitage \& Conner, 2001). Self-efficacy significantly and negatively predicted whether participants requested an earthquake planning guide in Study 2 and Study 3, supporting the exploration of this direct effect in the context of natural hazard preparation. Until a particular behaviour has been consistently demonstrated as not directly predicted by either or both factors of PBC, this association should be tested. The mixed findings for direct effects of self-efficacy on the different behaviour measures in Study 2 and Study 3 further suggest that the specific behaviour under examination could influence whether the direct effect exists or not.

This research tested the direct additive effect of PBC as recommended by Ajzen (2011a) and used in previous studies (Chow \& Chen, 2009; de Vries, Djikstra, \& Kuhlman, 1988). Ajzen argues that this direct design is more appropriate than a moderation design testing for an interaction between PBC and intention in predicting behaviour, and that this direct design provides a good account of the data (Ajzen, 1991). Of the minority of studies which test for an interaction, less than half show statistically significant results (Armitage \& Conner, 2001).

Behaviour measurement. Another potential explanation for the conflicting direct effects of PBC, and other TPB constructs overall, is the difference in behavioural measures. While self-efficacy predicted the specific, dichotomous behaviour of requesting an earthquake planning guide, it did not predict the measures of overall preparation. The evidence from these three different behaviour measures in Study 3 supports the recommendation that measures to assess intentions (and PBC) should be "compatible" (i.e., constructs refer to the same elements as each other) with the measures to assess behaviour (Ajzen, 2011a). Further, intentions to prepare for natural hazards predicted information seeking whereas intentions to prepare for 
natural disasters did not. Regardless of the positive or negative role of information seeking in increasing preparedness (see discussion above), the findings of Study 2, where the intentionbehaviour association differed depending on a specific wording change, demonstrates the importance of carefully considering how behaviour measures are constructed and from which previous research supporting evidence should be drawn.

Meta-analyses of TPB research which demonstrate some inconsistencies in how well intentions predict behaviour typically look at classes of behaviour, such as healthy eating (McDermott et al., 2015). However, studies included in such meta-analyses refer directly to "healthy eating" (Chan \& Tsang, 2011), or to specific actions such as "eat some fruit" (Hewitt \& Stephens, 2007), or to avoiding unhealthy food by following a "low fat diet" (Armitage \& Conner, 1999). While eating fruit and avoiding high-fat foods may share intended health outcomes and could be clustered within the same category of healthy eating, they are not the same behaviour. Such examples are particularly problematic as deliberately undertaking a behaviour and deliberately not undertaking a behaviour are cognitively dissimilar (Paton et al., 2005; Richetin, Conner, \& Perugini, 2011). To illustrate, McEachan et al. (2011) found that the TPB predicted physical activity better than it did diet behaviours; even though both share the same goal of improving health, they are different behaviours. The framing effects in Study 2 demonstrate that it is likely inappropriate to closely compare studies which, while ostensibly studying the same behaviour, either phrase that behaviour differently or in fact assess different actions. Future TPB studies could give more weight to previous findings from studies with identical wording of the target behaviour and could pilot test different wordings to identify potential framing effects.

Summary. This research offers several important contributions to the application of the Theory of Planned Behaviour. First, the findings support the inclusion of both the twofactor distinction (treating both theorized factors of each cognition component as distinct) and the two-level distinction (including indirect belief measures), including their use in evaluations of interventions both within and beyond the domain of natural hazard preparation. Second, this thesis adds to the growing recommendation for the use of structural equation modelling to test TPB models and provides an easily adapted questionnaire so that future studies can be as consistent as possible in terms of number and wording of scale items. Finally, this study contributes clarity to several issues around behaviour measurement and prediction. The findings of Studies 2 and 3 support the suggestion of a direct effect of self-efficacy on behaviour but qualified by a contextual caveat (e.g., relevance of self-efficacy to the particular behaviour). Study 2 also demonstrates meaningful impacts on TPB model results from small 
wording changes, suggesting that caution is necessary when comparing or reporting evidence from studies which phrase behavioural measures differently.

This focus on addressing inconsistencies in how the TPB is applied and measured is intended to help explain and unravel the confusion in previous findings but also to limit confusion in future research. Given the vast number of studies which have the same ultimate goal (e.g., finding ways to make people healthier: McDermott et al., 2015; McEachan et al., 2011), increasing the similarities between these studies in terms of the items used and the analyses conducted will allow for more meaningful comparisons of results. This in turn will allow for more confidence in supporting evidence for designing behaviour change interventions as well as more momentum in improving the theory beyond focusing on conceptual and methodological issues such as the ones addressed here.

\section{Natural Hazard Preparation}

The second main aim of the present research was to identify ways in which people can be encouraged to prepare for both direct impacts of natural hazards (i.e., mitigation actions) and resulting challenges during response and recovery (i.e., survival actions). Although survival actions are more commonly undertaken than mitigation actions (Russell et al., 1995; Spittal, McClure, Seigert, \& Walkey, 2008; Study 1 of this thesis), both types of preparation are still infrequent in many risk-prone areas including Wellington (Johnston et al., 2013; Study 1 of this thesis), New Zealand (Colmar-Brunton, 2018), Australia (Paton, 2019), and the United States (Bourque, 2013). The beliefs about preparing for natural hazards identified in this thesis are intended to inform the development of broad public campaigns, such as the annual ShakeOut drill, but can also be applied to targeted community interventions. Such an aim aligns with common recommendations for interventions to be theory-based (Ajzen, 2012; Hardeman et al., 2002; Joffe et al., 2016; Ronan et al., 2008; Tsorbatzoudis, 2005) and informed by recent and relevant understanding of why people do and do not prepare for the impacts of natural hazards (Ajzen, 2011a; Najafi et al., 2017).

The following discussion will briefly consider the implications of the framing effects found in Study 2, the barriers to behaviour identified in Study 1, and the components of the TPB not consistently associated with intentions to prepare in Study 2 and Study 3: controllability, perceived injunctive norms, and descriptive norms. Finally, I will discuss potential applications for the three factors significantly associated with intentions: instrumental attitudes, self-efficacy, and experiential attitudes.

Framing effects: Natural hazard versus natural disaster. The focus of this research is on preparation actions; that is, those taken before an event occurs. Message framing in other 
contexts, such as media reporting of actual events, can also be explored. It is important to refer to past natural hazard events that exceeded local capacities in a way that reflects this extensive impact so that such risks are taken seriously. Currently, the term disaster is used for this purpose, but this term does not imply the same meaning to the general public as the meaning which is inferred by researchers (Furedi, 2007). Further research could explore public interpretation of the term disaster used in this context, to ensure that media reports and government messaging, for example, are leading to the intended associations and understanding. That research could also explore differences between other common terms, such as emergency (used prominently by local, regional, and national government in New Zealand and internationally), in relation to preparedness. The other main consideration is whether it is better to refer to a specific hazard, such as an earthquake, or hazards broadly. The former has the benefit of being able to target both beliefs and preparation actions specific to that hazard but has the potential drawback of reducing awareness of hazards as often being cascading or compounding. That is, an earthquake can cascade and trigger a tsunami, landslides, and fires. Impacts of tsunami can be compounded by sea-level rise, landslides by heavy rain, and fires by drought or high wind. Communicating solely about earthquake potential may lead to inferences that this is the only hazard for which people need to prepare.

The test of framing effects in this thesis is preliminary, given the lack of similar research existing in the literature. While experimental surveys such as that used in Study 2 are useful for identifying framing effects (e.g., McClure, Allen, \& Walkey, 2001; McClure, Sutton, \& Sibley, 2007; McClure \& Velluppillai, 2013; Vinnell et al., 2018), qualitative methods could be used to complement such research. Once framing effects have been established in large samples, methods such as focus groups or interviews can use a small number of participants to gain a deeper understanding of how terms are perceived differently to explain the mechanisms of those framing effects.

Barriers to preparing. Study 1 replicated the findings of McClure et al. (2015) that people in Wellington are largely not preparing for natural hazards because they are either not thinking about it or they do not prioritize preparation. Several of the behaviours about which participants were asked have only recently started to be promoted, such as backing up important documents to cloud storage. These newer recommendations should continue so that people are more aware of a wider range of actions that they can take. However, many people are still not undertaking well-known preparation actions such as storing food and water or making an emergency plan. Study 1 showed that many people are not making these preparations mainly because they "haven't got around to it" or have not thought about taking a particular action, 
rather than based on factors such as low risk perception. This finding is useful for those attempting to encourage preparation by reducing the number of potential barriers they could target in an intervention. However, identifying why people do get around to preparing contributes more specific and therefore applicable information. This was the aim of Studies 2 and 3, and the cognitions and beliefs identified as significantly associated with intentions represent factors which lead to procrastination (i.e., not getting around to it). However, future work could employ qualitative methods to either complement the findings presented here or as part of a wider mixed methods approach. Given that participants in this research do largely view preparing as important and useful, it would be beneficial to gain a more thorough understanding of why those participants are not preparing using methods such as semistructured interviews which balance allowing participants to reflect at length on their own behaviour but with guidance from the researcher to ensure meaningful, subconscious influences are not missed.

While two of the cognitions found here to explain intentions to prepare (instrumental attitudes and self-efficacy) have been well-established in previous natural hazard research, the present research went further by identifying specific beliefs which lead to these attitudes and perceptions. Few studies have established such specific influences on behaviour so demonstrating the role of these specific beliefs represents an important and novel contribution of this research. Further, this research is among the first to empirically establish experiential attitudes as another determinant of people's intentions to prepare for natural hazards. This finding therefore also represents a novel and valuable contribution to the literature.

\section{Components not associated with intentions.}

Controllability. Controllability refers to perceptions of volition over the behaviour; that is, whether people believe they are able to choose to carry out (or not carry out) the behaviour. Ajzen (2011b) suggested that controllability and self-efficacy are both relevant only for some behaviours. The findings in this thesis suggest that controllability is not particularly relevant for natural hazard preparation. Limited research suggests that a sense of personal control over preparing is related to preparation (Becker et al., 2017). Some previous research has demonstrated that perceptions of personal responsibility to prepare increased household preparation (Arlikatti, Lindell, \& Prater, 2007; Becker et al., 2015) while perceptions that others, such as governments, are responsible inhibited household preparation (Flynn, Slovic, Mertz, \& Carlisle, 1999; Paton, Smith, \& Johnston, 2000). It is possible that the conflicting findings on responsibility reflect the different levels of preparation (e.g., government preparing infrastructure such as water network and individuals preparing their homes) identified in 
Chapter 1. Getting one's household ready might be more often seen as a personal responsibility, while people tend to hold government more responsible for larger-scale work such as strengthening vulnerable buildings and infrastructure (Flynn et al., 1999). While responsibility does not directly equate with controllability, the above research suggests that controllability perhaps was not relevant to participants' intentions in this study because those participants see household preparation as their responsibility and therefore by necessity under their control.

Social norms. Social norms refer to whether members of a social group engage in (descriptive norm) and approve of (injunctive norm) a particular behaviour (Cialdini, 2007). Previous research has repeatedly shown that these two types of norms are distinct (Hamann, Reese, Seewald, \& Loeschinger, 2015; Park \& Smith, 2007; Vinnell et al., 2018). Descriptive norms were positively and significantly associated with past behaviour in Study 1 and with intentions to prepare in Study 3 when the belief components were divided (see Figure 5.2) whereas injunctive norms were positively and significantly associated with intentions to prepare for natural disasters in Study 2 and intentions to prepare for earthquakes in Study 4. These findings suggest that both types of social norms do not have consistent roles regarding preparation for natural hazards.

Participants perceived positive injunctive norms among their social group, consistent with previous research demonstrating that Wellingtonians and people from other areas of New Zealand approve of preparing and think that most others also approve (Becker et al., 2014). However, the current number of people who are well prepared is low, which can lead to the belief that preparing would incur social judgments of being "over the top", "paranoid", or "crazy" (Becker et al., 2014; Cialdini, 2007). It is possible that the limited relevance of descriptive norms in the present research emerges from the accurate perception that preparation behaviour is low, while the relevance of injunctive norms may be limited by their conflict with descriptive norms. This suggestion is consistent with previous research demonstrating that the two types of norms have a greater influence on behaviour when they are congruent (Hamann, Reese, Seewald, \& Loeschinger, 2015; Smith et al., 2012; Vinnell et al., 2018). Norms function by providing cues on what behaviours are likely to be beneficial (Goldstein, Griskevicius, \& Cialdini, 2007) and allow individuals to avoid social punishment (Hamann et al., 2015).

Typically, to engage in a behaviour, people need to perceive that a majority of others similar to them are engaging in the behaviour and approve of it. However, minority norms presented as trending or dynamic norms have been used successfully to influence behaviour (Mortensen et al., 2017; Sparkman \& Walton, 2017). Trending norms convey that while a behaviour is currently undertaken by a minority of people this rate is increasing while dynamic 
norms convey that behavioural rates are shifting, so that they can also convey decreases in negative behaviours (e.g., meat consumption: Sparkman \& Walton, 2017); people are more likely to conform with a behaviour that is increasing in prevalence as they anticipate that behaviour becoming the norm (Mortensen et al., 2017). However, previous research does not show that preparation for natural hazards is increasing in Wellington or New Zealand at a sufficient rate to constitute a trending norm (Colmar-Brunton, 2018; Johnston et al., 2013). The communication of norms should be based on data rather than fabricated (Demarque, Charalambides, Hilton, \& Waroquier, 2015) and it is possible that people given information about a descriptive norm, even a trending one, which does not reflect reality will recognize the information as false and disregard it. Any efforts to change perceptions of social norms around natural hazard preparation should therefore do so carefully and empirically test whether those efforts are likely to meaningfully impact behaviour.

Norm effects might also be influenced by contextual factors. A recent meta-analysis of five earlier meta-analyses of norm effects on intentions and behaviour found that those effects differed based on country-level societal and cultural variables as well as between behavioural contexts (Fischer, Karl, \& Fischer, 2019). For example, this meta-analysis found that norm effects were weaker in countries that are more economically developed and more individualistic (such as New Zealand; Brougham \& Haar, 2013) than those which are less economically developed and more collectivistic. Such findings might suggest that comparing the norm effects found in this research to studies conducted in other countries is problematic; however, even if the strength of norm effects might vary across contexts, having accumulative applications of the TPB to preparation for natural hazards would clarify the importance (or not) of norms in this behavioural context.

A further potential explanation for the inconsistent effects of norm perceptions, both in this research and in the TPB literature broadly (Ajzen, 2011b), is the common argument that norms influence more automatic processes rather than more deliberative processes such as intention formation and subsequent behavioural decisions (Kredenster, Fabrigar, Smith, \& Fulton, 2012; Mortensen et al., 2017). Ajzen argues that norms can influence less deliberative actions at a level close to or below conscious awareness, and indeed people tend to underestimate the influence which norms have on their behaviour (Nolan et al., 2008). However, descriptive norms also tend to have stronger effects in new or ambiguous situations (Goldstein et al., 2007). Given the high levels of earthquake risk perception among people in Wellington (Johnston et al., 2013), it is likely that participants had information to draw on for their intention and behaviour decisions in this research other than their perceptions of social 
norms. This assumption implies that targeting social norms to increase natural hazard preparation may not be particularly beneficial. However, the findings discussed above, both from this thesis and previous research, suggest that norms may have a positive impact on preparation if the descriptive norm is brought into alignment with the injunctive norm. An example is convincing people that enough others are actually preparing that if they were to do the same they would not be perceived as "over the top" or abnormal.

Because of the complexity of these concepts, including specific considerations of context, norms should not be ruled out as useful intervention targets solely based on the lack of firm evidence in this research that they inform intentions to prepare. Such interventions should instead undertake thorough groundwork and theoretical development (Solberg et al., 2010). Overall, then, the recommendation of this thesis is that the role of social norms in preparation should not be ignored but that care is needed both in how they are examined and in how they are manipulated. Future research could employ qualitative or mixed methods approaches to ensure that the way in which norms are conceptualized and measured by the researcher corresponds with how participants understand and respond to social norm influences. It is possible that social norms play a meaningful role in the development of intentions to prepare but that the operationalization of norms and quantitative methods such as those used in this study are not sufficient for identifying such influences.

\section{Components associated with intentions.}

Instrumental attitudes. Instrumental attitudes refer to thoughts about the effect of a behaviour in terms of achieving the aim of that behaviour. This concept has not often been directly examined in the natural hazard literature but has clear conceptual overlap with two well-studied factors: outcome expectancy and response-efficacy (Becker et al., 2015). Many studies have identified these factors as important to disaster preparation (Becker et al., 2015; Hall \& Slothower, 2009; Johnston et al., 2013; Lindell \& Perry, 2000; Lindell \& Prater, 2000). Fatalism, the conceptual opposite, is also a key bias that inhibits preparation (e.g., McClure, Allen, \& Walkey, 2001; Paton et al., 2010; Terpstra \& Lindell, 2012). However, few studies have explored specific beliefs which precede these attitudes. Lindell, Arlikatti, and Prater (2009) examined attributes of specific actions, such as how maintaining a radio would protect "persons" or "property". In contrast, Study 3 demonstrated that those who had more positive instrumental attitudes believed that preparing (rather than a specific action) would both increase their chances of surviving a natural hazard event and that preparing would help them to generally get through the event better. 
These findings are encouraging for campaigns that focus on increasing either a number of specific actions or preparation generally as they show that people do not need to know the unique benefits and outcomes of specific actions. Instead, people are likely to prepare more if they are assured that preparing generally will be of benefit to them. Because this thesis focuses on general preparation at the household level, it did not include beliefs about specific behaviours. It is therefore important to note that researchers or practitioners aiming to encourage a single, specific action should still communicate the specific outcomes and benefits of that action. As informing such a specific application was not an aim of this thesis, that task is left to future research.

Instrumental attitudes can be targeted in two main ways: increasing perceptions of positive outcomes, or reducing perceptions of negative outcomes (Becker et al., 2015). The latter is particularly important for campaigns and interventions which include information about risks and impact; this information should focus on distinct, realistic damage which emphasizes the causal role of human actions (McClure et al., 2001; McClure et al., 2007; McClure \& Velluppillai, 2013) and should be paired with advice on what actions can be taken to improve outcomes (Becker et al., 2015). Tools such as testimonies from people who have benefited from their preparation can increase instrumental attitudes, particularly if those people are well-regarded by the target audience (Ajzen, 2011b).

Beliefs about the consequences of a behaviour can also be changed by providing information about the outcome, encouraging self-monitoring, and providing feedback (Michie, Johnston, Francis, Hardeman, \& Eccles, 2008). These tools are fairly vague and do not necessarily scale well to a public campaign but offer several possibilities for ways to apply the findings here to design new and adapt existing messaging. For example, the New Zealand Earthquake Commission website recommends a number of mitigation actions to take around the house. For each of these actions, the website comments on the benefits for reducing harm, damage, and inconvenience in a natural hazard event. The findings from Study 3 suggest that such messaging could be used for other types of preparation actions as well as in public campaigns which aim to encourage preparation without specifying particular behaviours.

Self-efficacy. Self-efficacy refers to whether people think they are capable of enacting a particular behaviour. This concept as used in this thesis has been studied extensively in the natural hazard domain (Becker et al., 2015; Johnston et al., 2013; Levac et al., 2012; Lindell \& Whitney, 2000; Paton, Millar, \& Johnston, 2001; Rowney, Farvid, \& Sibley, 2014). However, as with instrumental attitudes, this thesis extends these previous findings by identifying which specific beliefs inform self-efficacy. In contrast to findings from McClure et al. (2015) and 
Study 1 of this thesis, Study 3 found that participants' beliefs that they will think about preparing was not significantly associated with self-efficacy. However, in line with the other common barrier of simply not getting around to preparing, participants who believed more strongly that they will put in the effort to prepare had stronger intentions to do so than those who had a weaker belief in their ability to put in the effort. This finding suggests simple additions to future public campaigns or similarly simple changes to existing ones. Rather than (or as well as) emphasizing the low financial and time investment required for most recommended preparation actions, messaging can emphasize that these actions entail little effort. The National Emergency Management Agency in New Zealand recently ran a campaign emphasizing the low investment necessary for preparation (WREMOnz, n.d.). A number of posters presented the simple message that planning for an emergency is comparable to planning for common events such as a road trip, a festival, or a "Netflix binge" (Figure 7.1). 


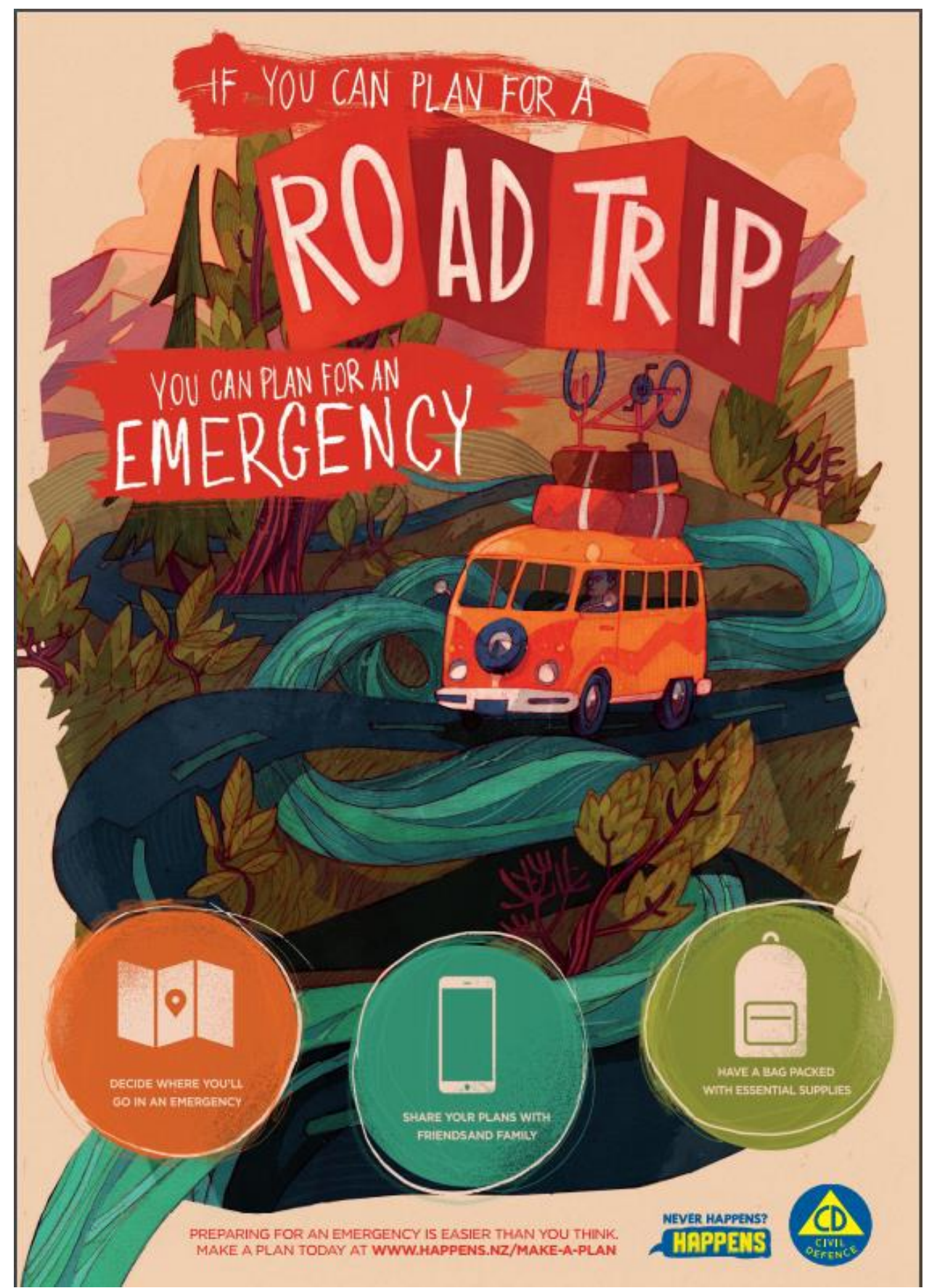

Figure 7.2. A poster as part of the National Emergency Management Agency's "Never Happens? Happens" campaign, comparing planning for an emergency to planning for another common behaviour, taking a road trip. Source: Wellington Region Emergency Management Office (WREMOnz) Facebook page. Retrieved from:

www.facebook.com/WREMOnz/photos/a.152527934776514/1805477409481550 
Such communications could also emphasize that many preparation actions require little effort and could go beyond the planning focus. Personalizing these advertisements to a specific demographic by targeting behaviours including road trips and watching Netflix as comparisons is an effective way to increase self-efficacy for that particular demographic (see e.g., Becker et al., 2015), but this strategy limits the audience which is likely to be impacted. Future campaigns could aim to make similar comparisons but without intentionally targeting a specific demographic or specific behaviour.

Several techniques have been used to increase people's beliefs about their capability to carry out a behaviour, including grading tasks, improving decision-making and coping skills, and social encouragement (Michie et al., 2008), which could be extended to increasing selfefficacy generally as well as specifically targeting the belief that preparing is too effortful. Finding a simple message which leads to an increase in coping skills is unlikely. However, existing campaigns demonstrate an awareness of the benefit of these techniques. For example, the earthquake planning guide used as a behaviour measure in Studies 2 and 3 splits preparation actions into different time frames: learning about hazard risks can be done "today", securing heavy objects "this weekend", and trying camping at home "next weekend". These tasks are roughly in order of effort so that people are encouraged to undertake easier actions first (i.e., grading tasks; Michie et al., 2008). Emphasizing the low amount of effort required for some of these actions is more likely to lead to the desired behaviour, which in turn has been shown to increase self-efficacy and therefore encourage further behaviour (Bandura, 1989; Rowney et al., 2014). Importantly, future campaigns should also include theoretically informed evaluations; no evaluation of this earthquake planning guide could be found within the public domain. Even when such campaigns are evaluated, the focus is typically on engagement rather than impacts on behaviour (Fraustino \& Ma, 2015).

Experiential attitudes. Experiential attitudes refer to people's judgments about the experience of enacting a particular behaviour. The present research demonstrated that experiential attitudes are significantly associated with intentions to prepare for a natural hazard, and specifically that both beliefs about the experience being fun and the experience being rewarding are relevant. To my knowledge, no previous study has shown that experiential attitudes relate to adults' preparation of their households for natural hazards. The programmes which do attempt to make learning about risk and preparation fun and rewarding tend to target children, rather than focusing on making conversations around risk and the task of preparing more appealing for adults (Bradley, McFarland, \& Clarke, 2014). For example, two research 
programmes in New Zealand test the use of toys $\left(\operatorname{Lego}^{\odot}{ }^{\text {bricks})}\right.$ and video games $\left(\right.$ Minecraft $^{\odot}$ ) to educate children on natural hazard risks and mitigation strategies (Dreaver, 2018). Limited research has used games to increase earthquake preparedness knowledge and self-efficacy among other populations, such as university students in the United States (Tanes \& Cho, 2013). While previous research shows that targeting children in efforts to increase both risk perception and preparation can spill over to influence the knowledge and behaviours of their parents (e.g., Ronan et al., 2008), more research is needed to identify ways to directly target adults' perceptions of natural hazard preparation as fun and rewarding.

One potential way to increase the perception that preparing is fun is to include humour in public messaging. Despite an increase in the inclusion of humour and pop-culture references in public campaigns to increase hazard preparation however, there is little evidence so far that such messages increase behaviour (Fraustino \& Ma, 2015). One campaign by the United States Centers for Disease Control and Prevention found lower intentions to prepare among those who received a message which related being prepared for disasters to being prepared for a zombie apocalypse compared to a group which received traditional messaging (Fraustino \& Ma, 2015). This campaign focused on risk and behaviour education, relying on humour to indirectly encourage uptake of those behaviours. It is possible that such an application of humour to the communication of the actual process of preparing, rather than risk, would increase experiential attitudes and therefore behaviour.

Few TPB studies have included experiential attitudes, and the majority of studies which report interventions apply the TPB as an evaluation tool rather than to inform the intervention itself (Hardeman et al., 2002). There is therefore scarce research on which to draw for potential ways to target this type of attitude towards preparation for natural hazards. Study 4 demonstrated more positive experiential attitudes among those who had participated in the 2018 ShakeOut drill than those who did not. This suggests that requiring actual action (practising "drop, cover, and hold") might make an intervention more fun. It is also possible that participants who have been able to undertake "drop, cover, and hold" in a real earthquake see their participation in the drill as more rewarding. This potential effect does overlap with instrumental attitudes but suggests that emphasizing how people benefit from preparing could make their attitudes to that behaviour more positive.

Summary. Despite decades of research to understand why people do and do not prepare for natural hazards (e.g., Lindell \& Perry, 2000; Solberg et al., 2010), there are still gaps in the literature for new and more specific factors which can be engaged with public campaigns. This research suggests that perceptions of personal control over preparing are not particularly 
relevant to intentions and that research to understand and influence social norms should do so carefully. While instrumental attitudes and self-efficacy have long been known to influence preparation (Becker et al., 2015; Lindell \& Perry, 2000), this thesis identifies novel specific beliefs which lead to these attitudes and perceptions. Logically, it is easier to convince people that preparing requires little effort than it is to convince people that they are capable of preparing as this latter task includes addressing other barriers such as time and cost (which did not relate to intentions to prepare in this research). Further, this research identifies the new factor of experiential attitudes as relevant to intentions to prepare. Knowing that people are more likely to prepare if they think the experience will be fun and rewarding offers many new opportunities for interventions.

Many interventions to change behaviour are currently not reported thoroughly in scientific papers so they cannot be effectively replicated (Joffe et al., 2016; Michie, Ashford, Sniehotta, Dombrowski, Bishop, \& French, 2011). Further, intervention studies in the area of natural hazards typically focus on risk communication and on evaluating small scale interventions such as workshops rather than public campaigns (Bradley et al., 2014). Although risk perception is necessary for preparation behaviour, it is not in itself a sufficient motivator (Bourque, 2013). Given the dearth of studies thoroughly reporting successful behaviour change interventions, let alone effects of public campaigns on preparation behaviour, there are few examples in the literature of ways in which to target the key factors identified in this thesis. Researchers and practitioners designing behaviour change campaigns ought to pilot test whatever tools they develop (McKenzie-Mohr, 2011). As the main aim of this thesis was to identify specific beliefs which relate to preparation, the task of identifying or developing and then testing ways to target these factors is left to future research. However, the clear establishment in this thesis that experiential attitudes relate to preparation, as well the identification of specific instrumental attitude and self-efficacy beliefs, represents novel and valuable knowledge to improve interventions to increase preparation for natural hazards. 


\section{Conclusion}

Natural hazards lead to billions of dollars of damage and harm millions of people around the world each year, with these impacts consistently increasing. New Zealand, like many other countries, is vulnerable to many natural hazards including earthquakes, tsunami, volcanic eruption, and high impact weather. While in some countries, government investment is helping to increase the resilience of our communities through efforts such as strengthening infrastructure, individuals need to be prepared to survive and respond to the impacts of hazard events. Understanding which cognitions and beliefs lead people to prepare their households for natural hazards is therefore an important endeavour to ensure better outcomes. A growing volume of research has explored these beliefs in Wellington (Johnston et al., 2020), New Zealand (Becker et al., 2015), and internationally (e.g., Solberg et al., 2010). While many of these previous studies have clearly established the role of factors such as risk perception and previous experience of hazard events, research identifying factors which can be more effectively targeted will help to improve the impact of public education campaigns.

Identifying such factors was one of the primary aims of this thesis. The findings show that natural hazard preparation is informed by the beliefs that people can make the effort to prepare, that preparation will help them survive and get through a hazard event better, and that preparing can be fun and rewarding. These findings offer several specific targets for efforts to encourage personal preparation. While a number of campaigns within New Zealand suggest some pre-existing awareness of the importance of these beliefs among emergency management practitioners, the specificity and empirical support offered by this research is valuable to improve the effectiveness of those campaigns.

This thesis achieved its primary aim of identifying beliefs important for natural hazard preparation using the framework of the Theory of Planned Behaviour (TPB). This theory is well-established within the field of social science, particularly the psychology of behaviour change. The research in this thesis suggests that the limited application of the TPB theory in the natural hazard domain is not a reflection of limited usefulness of the theory; both the variables found to explain intentions and those which did not offer important and novel insights as to why some people prepare while others do not.

As well as supporting the application of the TPB to predict natural hazard preparation, this thesis sought to address key inconsistencies within the TPB literature. The research presented here supports the inclusion of the two-factor distinction, the belief components, and careful consideration of the wording of the target behaviour. This research also offers a developed and tested scale which can be easily adapted for other behavioural contexts. The 
findings presented in this thesis can be used to make the methods used in TPB research more consistent which is likely to generate findings that can be compared and applied with greater confidence. This strategy will improve the effectiveness of interventions informed by the TPB.

In conclusion, this thesis makes meaningful contributions in terms of theoretical implications for research using the TPB as well as valuable knowledge to help people both in New Zealand and globally to get more prepared for the impacts of natural hazards. 


\section{References}

Adams, R. M., Karlin, B., Eisenman, D. P., Blakley, J., \& Glik, D. (2017). Who participates in the Great ShakeOut? Why audience segmentation is the future of disaster preparedness campaigns. International Journal of Environmental Research and Public Health, 14, pp.13. doi: 10.3390/ijerph14111407

Ajzen, I. (1991). The Theory of Planned Behavior. Organizational Behavior and Human Decision Processes, 50, 179-211. doi: 10.1016/0749-5978(91)90020-T

Ajzen, I. (2002). Perceived behavioral control, self-efficacy, locus of control, and the Theory of Planned Behavior. Journal of Applied Social Psychology, 32, 665-683. doi: 10.1111/j.1559-1816.2002.tb00236.x

Ajzen, I. (2006a). Behavioral interventions based on the Theory of Planned Behaviour [PDF file]. Retrieved from http://people.umass.edu/aizen/pdf/tpb.intervention.pdf

Ajzen, I. (2006b). Constructing a Theory of Planned Behavior questionnaire [PDF file]. Retrieved from https://people.umass.edu/aizen/pdf/tpb.measurement.pdf

Ajzen, I. (2011a). Behavioral interventions: Design and evaluation guided by the Theory of Planned Behavior. In M. M. Mark, S. I. Donaldson, \& B. Campbell (Eds.), Social psychology and evaluation (pp. 72-101). New York, US: Guilford Press.

Ajzen, I. (2011b). The Theory of Planned Behaviour: Reactions and reflections. Psychology \& Health, 26, 1113-1127. doi: 10.1080/08870446.2011.613995

Ajzen, I. (2012). The Theory of Planned Behavior. In P. A. M. Lange, A. W. Kruglanski, \& E. T. Higgins (Eds.), Handbook of theories of social psychology (Vol. 1, pp. 438-459). London, UK: Sage.

Ajzen, I. (2013). Constructing a Theory of Planned Behavior questionnaire [PDF file]. Retrieved from: http://www.midss.org/sites/default/files/tpb.construction.pdf

Ajzen, I., \& Fishbein, M. (2004). Questions raised by a reasoned action approach: Comment on Ogden (2003). Health Psychology, 23, 431-434. doi: 10.1037/0278-6133.23.4.431

Ajzen, I., \& Madden, T. J. (1986). Prediction of goal-directed behavior: Attitudes, intentions, and perceived behavioral control. Journal of Experimental Social Psychology, 22, 453 474. doi: 10.1016/0022-1031(86)90045-4

Alexander, D., Gaillard, J.C., Kelman, I., Marincioni, F., Penning-Rowsell, E., van Niekerk, D., \& Vinnell, L. J. (2020). Academic publishing in disaster risk reduction: past, present, and future. Disasters. Advance online publication. doi: 10.1111/disa.12432 
Arau'jo-Soares, V., McIntyre, T., \& Sniehotta, F.F. (2009). Predicting changes in physical activity amongst adolescents: The role of self-efficacy, intention, action planning and coping planning. Health Education Research, 24, 128-139. doi: 10.1093/her/cyn005

Arlikatti, S., Lindell, M. K., \& Prater, C. S. (2007). Perceived stake-holder role relationships and adoption of seismic hazard adjustments. International Journal of Mass Emergencies and Disasters, 25(3), 218-256.

Armitage, C. J., \& Conner, M. (1999). Distinguishing perceptions of control from self-efficacy: Predicting consumption of a low fat diet using the theory of planned behaviour. Journal of Applied Social Psychology, 29, 72-90. doi: 10.1111/j.1559-1816.1999.tb01375.x

Armitage, C. J., \& Conner, M. (2001). Efficacy of the Theory of Planned Behaviour: A metaanalytic review. British Journal of Social Psychology, 40, 471-499.

Armitage, C. J., Reid, J. C., \& Spencer, C. P. (2013). Changes in cognition and behaviour: a causal analysis of single-occupancy car use in a rural community. Transportmetrica A: Transport Science, 9, 1-10. doi: 10.1080/18128602.2010.509706

Åstrøm, A. N., \& Okullo, I. (2004). Temporal stability of the Theory of Planned Behaviour: A prospective analysis of sugar consumption among Ugandan adolescents. Community Dentistry and Oral Epidemiology, 32, 426-434. doi: 10.1111/j.16000528.2004.00186.x

Ballantyne, M., Paton, D., Johnston, D., Kozuch, M., \& Daly, M. (2000). Information on volcanic and earthquake hazards: The impact on awareness and preparation. Institute of Geological and Nuclear Sciences Report, 2000(2). 45 p.

Bandura, A. (1986). Social foundations of thought and action: A social cognitive theory. Englewood Cliffs, NJ: Prentice-Hall, Inc.

Bandura, A. (1989). Social Cognitive Theory. In R. Vasta (Ed.), Annals of child development. Vol. 6. Six theories of child development (pp. 1-60). Greenwich, CT: JAI Press.

Bates, B. R., Quick, B. L., \& Kloss, A. A. (2009). Antecedents of intention to help mitigate wildfire: Implications for campaigns promoting wildfire mitigation to the general public in the wildland-urban interface. Safety Science, 47, 374-381. doi: 10.1016/j.ssci.2008.06.002

Beatson, R., \& McLennan, J. (2011). What applied social psychology theories might contribute to community bushfire safety research after Victoria's "Black Saturday". Australian Psychologist, 46, 171-182. doi: 10.1111/j.1742-9544.2011.00041.x

Becker, J. (2009). Observations from the Great Southern California Earthquake ShakeOut. GNS Science Report, 2009(31). 24 p. 
Becker, J. S., Paton, D., Johnston, D. M. (2015). Communication of risk: A community resilience perspective. GNS Science Report 2015/66. 30 p.

Becker, J., Paton, D., Johnston, D., \& Ronan, K. (2014). Societal influences on earthquake information meaning-making and household preparedness. International Journal of Mass Emergencies and Disasters, 32(2), 317-352.

Becker, J. S., Paton, D., Johnston, D. M., Ronan, K. R., \& McClure, J. (2017). The role of prior experience in informing and motivating earthquake preparedness. International Journal of Disaster Risk Reduction, 22, 179-193. doi: 10.1016/j.ijdrr.2017.03.006

Benjamin, D., Por, H., \& Budescu, D. (2017). Climate change versus global warming: Who is susceptible to the framing of climate change? Environment and Behavior, 49, 745-770. doi: 10.1177/0013916516664382

Blake, D., Marlowe, J., \& Johnston, D.M. (2017). Get prepared: Discourse for the privileged? International Journal of Disaster Risk Reduction, 25, 283-288. doi: 10.1016/j.ijdrr.2017.09.012

Blake, D., Tipler, K., Garden, E., Johnston, D.M., \& Becker, J.S. (2018). Wellington household preparedness survey. GNS Science report 2018/23, 109 p. doi: 10.21420/G2GH10

Blanchard, K. (2018, November 16). \#NoNaturalDisasters - Changing the discourse of disaster reporting. Retrieved from www.preventionweb.net/experts/oped/view/61996

Block, L. G., \& Keller, P. A. (1995). When to accentuate the negative: The effects of perceived efficacy and message framing on intentions to perform a health-related behaviour. Journal of Marketing Research, 32(2), 192-203.

Boomsma, A. (1985). Nonconvergence, improper solutions, and starting values in LISREL maximum likelihood estimation. Psychometrika, 50, 229-242. doi: 10.1007/BF02294248

Bourque, L. (2013). Household preparedness and mitigation. International Journal of Mass Emergencies and Disasters, 31(3), 360-372.

Bourque, L. B., Regan, R., Kelley, M. M., Wood, M. M., Kano, M., \& Mileti, D. S. (2012). An examination of the effect of perceived risk on preparedness behavior. Environment and Behavior, 45(5), 615-649. doi: 10.1177/0013916512437596

Bradley, D. T., McFarland, M., \& Clarke, M. (2014). The effectiveness of disaster risk communication: A systematic review of intervention studies. PLoS Currents Disasters, 6, 22p. doi: 10.1371/currents.dis.349062e0db1048bb9fc3a3fa67d8a4f8 
Breitsohl, H. (2018). Beyond ANOVA: An introduction to Structural Equation Models for experimental designs. Organizational Research Methods, 22(3), 649-677. doi: $10.1177 / 1094428118754988$

Brenkert-Smith, H., Champ, P. A., \& Flores, N. (2012). Trying not to get burned: Understanding homeowners' wildfire risk-mitigation behaviors. Environmental Management, 50, 1139-1151. doi: 10.1007/s00267-012-9949-8

Brougham, D., \& Haar, J. M. (2013). Collectivism, cultural identity and employee mental health: A study of New Zealand Māori. Social Indicators Research, 114(3), 1143-1160. doi: 10.1007/s11205-012-0194-6

Brown, C., McDonald, G., Uma, S. R., Smith, N., Sadashiva, V., Buxton, R., ... Daly, M. (2019). From physical disruption to community impact: modelling a Wellington Fault earthquake. Australasian Journal of Disaster and Trauma Studies, 23(2), 65-76.

Brun, W. (1992). Cognitive components in risk perception: Natural versus manmade risks. Journal of Behavioral Decision Making, 5, 117-132. doi: 10.1002/bdm.3960050204

Brundl, M., Romang, H. E., Bischof, N., \& Rheinberger, C. M. (2009). The risk concept and its application in natural hazard risk management in Switzerland. Natural Hazards and Earth System Sciences, 9, 801-813. doi: 10.5914/nhess-9-801-2009

Bubeck, P., Botzen, W. J. W., Laudan, J., Aerts, J. C. J. H., \& Thieken, A. H. (2017). Insights into flood-coping appraisals of Protection Motivation Theory: Empirical evidence from Germany and France. Risk Analysis, 38(6), 1239-1275. doi: 10.1111/risa.12938

Building (Earthquake-prone Buildings) Amendment Bill 2013. Retrieved from www.legislation.govt.nz/bill/government/2013/0182/latest/DLM5616102.html

Byrne, B. M. (2012). Structural equation modelling with Mplus. New York, US: Routledge.

Chan, K., \& Tsang, L. (2011). Promote healthy eating among adolescents: a Hong Kong study. Journal of Consumer Marketing, 28, 354-362. doi: 10.1108/07363761111150008

Chen, Y., \& Hong, Y. (2015). Different ways to resolve discrepancy between descriptive and injunctive norms across cultures. Journal of Cross-Cultural Psychology, 46(10), 13161319. doi: 10.1177/0022022115600265

Cheung, G. W., \& Lau, R. S. (2011). A direct comparison approach for testing measurement invariance. Organizational Research Methods, 15(2), 167-198. doi: $10.1177 / 1094428111421987$

Chmutina, K., \& von Meding, J. (2019). A dilemma of language: "Natural disasters" in academic literature. International Journal of Disaster Risk Science, 10, 283-292. doi: 10.1007/s13753-019-00232-2 
Chow, W. S., \& Chen, Y. (2009). Intended belief and actual behavior in green computing in Hong Kong. Journal of Computer Information Systems, 50(2), 136-141. doi: $10.1080 / 08874417.2009 .11645392$

Cialdini, R. B. (2007), Descriptive social norms as underappreciated sources of social control. Psychometrika, 72(2), 263-268.

Cialdini, R. B., Reno, R. R., \& Kallgren, C. A. (1990). A focus theory of normative conduct: Recycling the concept of norms to reduce littering in public places. Journal of Personality and Social Psychology, 58 (6), 1015-1026.

Colmar Brunton Ltd. (2018). Campaign Monitoring Research 2018. National Emergency Management Agency: Wellington, New Zealand.

Conner, M., Hugh-Jones, S., \& Berg, C. (2011). Using the two-factor Theory of Planned Behaviour to predict adolescent breakfast choices. Educational \& Child Psychology, 28(4), 37-50. doi: 10.1186\%2Fs12966-015-0324-Z

Coomer, M. A., Tarrant, R. A. C., Hughes, M. E., \& Johnson, V. (2012). An earthquake emergency response an evacuation exercise in a New Zealand school: A 2011 case study report. GNS Science report 2012/03, 16 p.

Cooper-Cabell, N. (2013). Mind the gap: Post earthquake community wellbeing? Aotearoa New Zealand Social Work, 25(2), 27-34.

Crawford, M. H., Saunders, W. S. A., Doyle, E. E. H., Leonard, G. S., \& Johnston, D. M. (2019). The low-likelihood challenge: Risk perception and the use of risk modelling for destructive tsunami policy development in New Zealand local government. Australasian Journal of Disaster and Trauma Studies, 23(1), 3-20.

Daly, M. (2018, February 21). Former cyclone Gita crosses New Zealand, leaving destruction in its wake. Stuff. Retrieved from www.stuff.co.nz

Darker, C. D., French, D. P., Longdon, S., Morris, K., \& Eves, F. F. (2007). Are beliefs elicited biased by question order? A Theory of Planned Behaviour belief elicitation study about walking in the UK general population. British Journal of Health Psychology, 12, 93110. doi: $10.1348 / 135910706 \times 100458$

Davison, C. (1934). The Hawke's Bay earthquake of February 3, 1931. Nature, 133, 841-842. doi: $10.1038 / 133841 \mathrm{a} 0$

Demarque, C., Charalambides, L., Hilton, D. J., \& Waroquier, L. (2015). Nudging sustainable consumption: The use of descriptive norms to promote a minority behavior in a realistic online shopping environment. Journal of Environmental Psychology, 43, 166-174. doi: 10.1016/j.jenvp.2015.06.008 
de Vries, H., Djikstra, M., \& Kuhlman, P. (1988). Self-efficacy: the third factor besides attitude and subjective norm as a predictor of behavioural intentions. Health Education Research, 3(3), 273-282. doi: 10.1093/her/3.3.273

Dillman, D. A., Smyth, J. D., \& Christian, L. M. (2014). Internet, phone, mail, and mixed mode surveys: The tailored design method. Hoboken, NJ: John Wiley \& Sons.

Doyle, E. H., McClure, J., Potter, S. H., Becker, J. S., Johnston, D. M., Lindell, M. K., ... Coomer, M. A. (2018). Motivations to prepare after the 2013 Cook Strait earthquake, N.Z. International Journal of Disaster Risk Reduction, 31, 637-649. doi: 10.1016/j.ijdrr.2018.07.008

Doyle, E. E. H., Lambie, E., Orchiston, C., Becker, J. S., McLaren, L., Johnston, D., \& Leonard, G. (2020). Citizen science as a catalyst for community resilience building: A two-phase tsunami case study. Australasian Journal of Disaster and Trauma Studies, 24, 23-49.

Dreaver, C. (2018, August 5). In case of emergency: Children map community using Lego and Minecraft. Radio New Zealand. Retrieved from rnz.co.nz

Dufty, N. (2009). Natural hazards education in Australian schools: How can we make it more effective? The Australian Journal of Emergency Management, 24(2), 13-16.

Duval, T. S., \& Mulilis, J. (1999). A Person-relative-to-Event (PrE) approach to negative threat appeals and earthquake preparedness: A field study. Journal of Applied Social Psychology, 29, 495-516. doi: 10.1111/j.1559-1816.1999.tb01398.x

Eisinga, R., Grotenhuis, Mt., \& Pelzer, B. (2013). The reliability of a two-item scale: Pearson, Cronbach, or Spearman-Brown? International Journal of Public Health, 58, 637-642. doi: 10.1007/s00038-012-0416-3

Ejeta, L. T., Ardalan, A., \& Paton, D. (2015). Application of behavioral theories to disaster and emergency health preparedness: A systematic review. PLOS Currents Disasters. doi: 10.1371/currents.dis.31a8995ced321301466db400f1357829.

Elliott, M. A., \& Armitage, C. J. (2009). Promoting drivers' compliance with speed limits: Testing an intervention based on the Theory of Planned Behaviour. British Journal of Psychology, 100, 111-132. doi: 10.1348/000712608X318626

Elms, D. (2015). Improving community resilience to natural events. Civil Engineering and Environmental Systems, 32(1-2), 77-89. doi: 10.1080/10286608.2015.1011626

Farrow, K., Grolleau, G., \& Ibanez, L. (2017). Social norms and pro-environmental behavior: a review of the evidence. Ecological Economics, 140, 1-13. doi: Organizational Behavior and Human Decision Processes, 127, 59-69. 10.1016/j.ecolecon.2017.04.017 
Fennis, B. M., Adriaanse, M. A., Stroebe, W., \& Pol, B. (2011). Bridging the intentionbehavior gap: Inducing implementation intentions through persuasive appeals. Journal of Consumer Psychology, 21, 302-311. doi: 10.1016/j.jcps.2010.12.003

Fife-Schaw, C., Sheeran, P., \& Norman, P. (2007). Simulating behaviour change interventions based on the theory of planned behaviour: Impacts on intention and action. British Journal of Social Psychology, 46, 43-68. doi 10.1348/014466605X85906

Finnis, K. K. (2007). From science to practice: Community-based public education initiatives. Wellington, New Zealand: Joint Centre for Disaster Research.

Finucane, M. L., Slovic, P., Mertz, C. K., Flynn, J., \& Satterfield, T. A. (2000). Gender, race and perceived risk: the 'white male' effect. Health, Risk \& Society, 2(2), 159-172.

Fischer, R., \& Karl, J. A. (2019). A primer to (cross-cultural) multi-group invariance testing possibilities in R. Frontiers in Psychology, 18 p. doi: 10.3389/fpsyg.2019.01507

Fischer, R., Karl, J. A., Fischer, M. V. (2019). Norms across cultures: A cross-cultural metaanalysis of norms effects in the Theory of Planned Behavior. Journal of Cross-Cultural Psychology, 50, 1112-1126. doi: 10.1177/0022022119846409

Fishbein, M., \& Ajzen, I. (1975). Belief, attitude, intention and behavior: An introduction to theory and research. Reading, MA: Addison-Wesley

Fisher, R. J. (1993). Social desirability bias and the validity of indirect questioning. Journal of Consumer Research, 20(2), 303-315. doi: 10.1086/209351

Flusberg, S. J., Matlock, T., \& Thibodeau, P. H. (2017). Metaphors for the war (or race) against climate change. Environmental Communication, 11, 769-783. doi: 10.1080/17524034. 2017.1289111

Flynn, J., Slovic, P., Mertz, C. K., \& Carlisle, C. (1999). Public support for earthquake risk mitigation in Portland, Oregon. Risk Analysis, 19(2), 205-216.

Francis, J. J., Eccles, M. P., Johnston, M., Walker, A., Grimshaw, J., Foy, R., ... Bonetti, D. (2004). Constructing questionnaires based on the Theory of Planned Behaviour: A manual for health services researchers. Centre for Health Services Research: Newcastle upon Tyne, UK.

Fraser, S.A., Doyle, E.E.H., Wright, K.C., Potter, S.H., McClure, J., Johnston, D.M., ... Johal S. (2016) Tsunami response behaviour during and following two local-source earthquakes in Wellington, New Zealand. International Journal of Disaster Risk Reduction, 16, 123-133. doi: 10.1016/j.ijdrr.2016.02.008 
Fraustino, J. D., \& Ma, L. (2015). CDC's use of social media and humor in a risk campaign "Preparedness 101: Zombie Apocalypse. Journal of Applied Communication Research, 43(2), 222-241. doi: 10.1080/00909882.2015.1019544

Furedi, F. (2007). The changing meaning of disaster. Area, 39, 482-489. doi: 10.1111/j.1475 4762.2007.00764.x

Gampell, A. V., Gaillard, J. C., Parsons, M., \& Le Dé, L. (2020). Exploring the use of the Quake Safe House video game to foster disaster and disaster risk reduction awareness in museum visitors. International Journal of Disaster Risk Reduction, 49, 1-10. doi: 10.1016/j.ijdrr.2020.101670

Gelo, O., Braakmann, D., \& Benetka, G. (2008). Quantitative and qualitative research: Beyond the debate. Integrative Psychological and Behavioral Science, 42(3), 266-290. doi: 10.1007/s12124-008-9078-3

George, D. (2017, March 24). Major earthquake could split Wellington region into 'seven islands'. Stuff. Retrieved from www.stuff.co.nz

George, D. (2018, December 14). Saving Wellington: Civil Defence lays out plan to get capital back on its feet after major earthquake. Stuff. Retrieved from www.stuff.co.nz

Giles, M., McClenahan, C., Armour, C., Millar, S., Rae, G., Mallett, J., \& Stewart-Knox, B. (2014). Evaluation of a Theory of Planned Behaviour-based breastfeeding intervention in Northern Irish schools using a randomized cluster design. British Journal of Health Psychology, 19, 16-35. doi: 10.1111/bjhp.12024

Glik, D. C., Eisenman, D. P., Zhou, Q., Tseng, C-H., \& Asch, S. M. (2014). Using the Precaution Adoption Process model to describe a disaster preparedness intervention among low-income Latinos. Health Education Research, 29(2), 272-283. doi: 10.1093/her/cyt109

Goldstein, N. J., Griskevicius, V., \& Cialdini, R. B. (2007). Invoking social norms: A social psychology perspective on improving hotels' linen-re-use programs. Cornell Hotel and Restaurant Administration Quarterly, 48(2), 145-150.

Gollwitzer, P. M. (1999). Implementation intentions: Strong effects of simple plans. American Psychologist, 54, 493-503. doi: 10.1037/0003-066X.54.7.493

Gowan, M.E., Kirk, R.C., \& Sloan, J.A. (2014). Building resiliency: A cross-sectional study examining relationships among health-related quality of life, well-being, and disaster preparedness. Health and Quality of Life Outcome, 12(85), 1-17. doi: 10.1186/1477$7525-12-85$ 
Gregorich, S. E. (2007). Do self-report instruments allow meaningful comparisons across diverse population groups? Testing measurement invariance using the confirmatory factor analysis framework. Medical Care, 44, 78-94. doi: 10.1097/01.mlr.0000245454. $12228.8 \mathrm{f}$

Griffin, R. J., Dunwoody, S., \& Neuwirth, K. (1999). Proposed model of the relationship of risk information seeking and processing to the development of preventive behaviors. Environmental Research, 80, 230-245. doi: 10.1006/enrs.1998.3940

Guo, R., Berkshire, S. D., Fulton, L. V., \& Hermanson, P. M. (2017). Predicting intention to use evidence-based management among U.S. healthcare administrators: Application of the Theory of Planned Behavior and structural equation modelling. International Journal of Healthcare Management, 8 p. doi: 10.1080/20479700.2017.1336856

Guo, J. L., Wang, R. N., Liao, J. Y., \& Huang, C. M. (2016). Efficacy of the Theory of Planned Behavior in predicting breastfeeding: Meta-analysis and structural equation modeling. Applied Nursing Research, 29, 37-42. doi: 10.1016/j.apnr.215.03.016

Hall, T. E., \& Slothower, M. (2009). Cognitive factors affecting homeowners' reactions to defensible space in the Oregon coast range. Society and Natural Resources, 22, 95-110. doi: 10.1080/08941920802392187

Hallegatte, S., Rentschler, J., \& Rozenberg, J. (2019). Lifelines: The resilience infrastructure opportunity. Washington, DC: World Bank Group.

Hamann, K. R. S., Reese, G., Seewald. D., \& Loeschinger, D. C. (2015). Affixing the theory of normative conduct (to your mailbox): Injunctive and descriptive norms as predictors of anti-ads sticker use. Journal of Environmental Psychology, 44, 1-9. doi: 10.1016/j.envp.2015.08.003

Hardeman, W., Johnston, M., Johnston, D., Bonetti, D., Wareham, N., \& Kinmonth, A. L. (2002). Application of the Theory of Planned Behaviour in behaviour change interventions: A systematic review. Psychology \& Health, 17, 123-158. doi: $10.1080 / 08870440290013644 a$

Heath, R. L., Lee, J., Palenchar, M. J., \& Lemon, L. L. (2018). Risk communication emergency response preparedness: Contextual assessment of the Protective Action Decision Model. Risk Analysis, 38, 333-344. doi: 10.1111/risa.12845

Heinrichs, N., Rapee, R. M., Alden, L. A., Bogels, S., Hofmann, S. G., Oh, K. J., \& Sakano, Y. (2006). Cultural differences in perceived social norms and social anxiety. Behaviour Research and Therapy, 44, 1187-1197. doi: 10.1016/j.brat.2005.09.006 
Heller, K., Alexander, D. B., Gatz, M., Knight, B. G., \& Rose, T. (2006). Social and personal factors as predictors of earthquake preparation: The role of support provision, network discussion, negative affect, age, and education. Journal of Applied Social Psychology, 35(2), 399-422. doi: 10.1111/j.1559-1816.2005.tb02127.x

Henrich, L., McClure, J., \& Crozier, M. (2015). Effects of risk framing on earthquake risk perception: Life-time frequencies enhance recognition of the risk. International Journal of Disaster Risk Reduction, 13, 145-150. doi: 10.1016/j.ijdrr.2015.05.003

Henrich, L., McClure, J., \& Doyle, E. E. H. (2018). Perceptions of risk characteristics of earthquakes compared to other hazards and their impact on risk tolerance. Disasters, 42, 761-781. doi: 10.1111/disa. 12284

Hewitt, A. M., \& Stephens, C. (2007). Healthy eating among 10 - 13-year-old New Zealand children: Understanding choice using the Theory of Planned Behaviour and the role of parental influence. Psychology, Health \& Medicine, 12, 526-535. doi: $10.1080 / 13548500601164396$

Hobfoll, S. E., Stevens, N. R., \& Zalta, A. K. (2015). Expanding the science of resilience: Conserving resources in the aid of adaptation. Psychological Inquiry: An International Journal for the Advancement of Psychological Theory, 26(2), 174-180. doi: 10.1080/1047840X.2015.1002377

Hofstede Insights (n.d.). Country comparison. Accessed $13^{\text {th }}$ July 2020 at www.hofstedeinsights.com/country-comparison

Horspool, N., Cousins, W. J., \& Power, W. L. (2015). Review of tsunami risk facing New Zealand: A 2015 update. GNS Science Consultancy Report 2015(38). 52 p.

Houts, P. S., Lindell, M. K., Hu, T. W., Cleary, P. D., Tokuhata, G., \& Flynn, C. B. (1984). Protective Action Decision Model applied to evacuation during the Three Mile Island crisis. International Journal of Mass Emergencies and Disasters, 2, 27-39.

id. (n.d.). Wellington City ethnic groups. Accessed $13^{\text {th }}$ July 2020 at https://profile.idnz.co.nz/wellington/ethnic-group

InternetNZ. (2017). State of the Internet 2017 [PDF file]. Retrieved from https://internetnz.nz/state-internet-report-2017

Jang, L-J., Wang, J.J., Paton, D., \& Ning-Yu, T. (2016). Cross-cultural comparisons between the earthquake preparedness models of Taiwan and New Zealand. Disasters, 40, 327-345. 
Joffe, H., Perez-Fuentes, G., Potts, H. W.W., \& Rossetto, T. (2016). How to increase earthquake and home fire preparedness: the fix-it intervention. Natural Hazards, 84, 1943-1965. doi: 10.1007/s11069-016-2528-1

Johnson, V. A., Johnston, D. M., Ronan, K. R., \& Peace, R. (2014). Evaluating children's learning of adaptive response capacities from ShakeOut, an earthquake and tsunami drill in two Washington State school districts. Homeland Security \& Emergency Management, 11, 347-373. doi: 10.1515/jhsem-2014-0012

Johnston, D., Becker, J., McClure, J., Paton, D., McBride, S., Wright, K., ... Hughes, M. (2013). Community understanding of, and preparedness for, earthquake and tsunami risk in Wellington, New Zealand. In H. Joffe, T. Rossetto \& J. Adams (Eds.), Cities at risk: Living with perils in the $21^{\text {st }}$ century (pp. 131-148). Springer. doi: 10.1007/97894-007-6184-1_8

Johnston, D.M., Standring, S., Ronan, K., Lindell, M., Wilson, T., Cousins, W.J., ... Bissell, R. (2014). The 2010/2011 Canterbury earthquakes: context and cause of injury. Natural Hazards, 73(2), 627-637. doi: 10.1007/s11069-014-1094-7

Johnston, D., Tarrant, R., Tipler, K., Coomer, M., Pedersen, S., \& Garside, R. (2011). Preparing schools for future earthquakes in New Zealand: Lessons from an evaluation of a Wellington school exercise. Australian Journal of Emergency Management, 26(1), 2430.

Johnston, D., Tarrant, R., Tipler, K., Lambie, E., Crawford, M., Johnson, V., ... Ronan, K. (2016). Towards tsunami-safer schools in the Wellington region of New Zealand: Evaluating drills and awareness programs. Australian Journal of Emergency Management, 31(3), 59-66.

Johnston, D.M., Vinnell, L.J., Wallis, A., Coomer, M.A., McClure, J., Becker, J.S., Gunnell, S. (2020). A bibliography of social research on the earthquake risk in Wellington, Aotearoa New Zealand: 1848 to 2019. Disaster Research Science Report, 2020/02, Wellington (New Zealand): Massey University. 49 p.

Kahlor, L. A. (2010). PRISM: A planned risk information seeking model. Health Communication, 25, 345-356. doi:10.1080/10410231003775172

Kahlor, L. A., Olson, H. C., Markman, A. B., \& Wang, W. (2018). Avoiding trouble: Exploring environmental risk information avoidance intentions. Environment and Behavior, 52(2), 187-28. doi: 10.1177/0013916518799149 
Karimi-Shahanjarini, A., Rashidian, A., Omidvar, N., \& Majdzadeh, R. (2013). Assessing and comparing the short-term effects of TPB only and TPB plus implementation intentions interventions on snacking behavior in Iranian adolescent girls: A cluster randomized trial. American Journal of Health Promotion, 27(3), 152-161. doi: 10.4278/ajhp.110311-QUAN-113

Kaye, N. (2016). \$2.5m public education investment to improve Kiwi's preparedness for emergencies. Retrieved from www.beehive.govt.nz/release/25m-public-educationinvestment-improve-kiwis\%E2\%80\%99-preparedness-emergencies

Kellet, J., \& Caravani, A. (2013). Financing disaster risk reduction: A 20 year story of international aid. Overseas Development Institute and Global Facility for Disaster Reduction and Recovery: London, UK.

Kelman, I. (2018). Lost for words amongst disaster risk science vocabulary? International Journal for Disaster Risk Science, 9, 281-291. doi: 10.1007/s13753-018-0188-3

Kennedy, C., Hatley, N., Lau, A., Mercer, A., Keeter, S., Ferno, J., \& Asare-Marfo, D. (2020). Assessing the risks to online polls from bogus respondents. Pew Research Center.

Khan, S., Crozier, M.J., \& Kennedy, D. (2012). Influences of place characteristics on hazards, perception and response: A case study of the hazardscape of the Wellington Region, New Zealand. Natural Hazards, 62(2), 501-529. doi:10.1007/s11069-012-0091-y

King, D. N. T., Goff, J., \& Skipper, A. (2007). Māori environmental knowledge and natural hazards in Aotearoa-New Zealand. Journal of the Royal Society of New Zealand, 37(2), 59-73. doi: 10.1080/03014220709510536

Kos, P. (2010). Earthquake risk assessment - Wellington Region. Great Wellington Regional Council. http://www.gw.govt.nz/assets/councilreports/Report_PDFs/2010_567_1_ Report.pdf

Kothe, E. J., Ling, M., North, M., Klas, A., Mullan, B. A., \& Novoradovskaya, L. (2019). Protection Motivation Theory and pro-environmental behaviour: A systematic mapping review. Australian Journal of Psychology, 71, 411-432. doi: 10.1111/ajpy.12271

Kredenster, M. S., Fabrigar, L. R., Smith, S. M., \& Fulton, K. (2012). Following what people think we should do versus what people actually do: Elaboration as a moderator of the impact of descriptive and injunctive norms. Social Psychological and Personality Science, 3(3), 341-347. doi: 10.1177/1948550611420481 
Kwok, A.H., Becker, J., Paton, D., Doyle, E.H., \& Johnston, D. (2019). Stakeholders' perspectives of social capital in informing the development of neighbourhood-based disaster resilience measurements. Journal of Applied Social Science, 13, 26-57. doi: $10.1177 / 1936724419827987$

Lake, B., Milfont, T. L., \& Gavin, M. (2011). The relative influence of psycho-social factors on urban edible gardening. New Zealand Journal of Psychology, 40(3), 50-59.

Lambie, E.S., Wilson, T.M., Brogt, E., Johnston, D.M., Ardagh, M., Deely, J., ... FeldmannJensen, S. (2017). Closed Circuit Television (CCTV) earthquake behaviour coding methodology: Analysis of Christchurch Public Hospital video data from the 22 February Christchurch earthquake event. Natural Hazards, 86(3), 1175-1192. doi: $10.1007 / \mathrm{s} 11069-016-2735-9$

Landrum, B., \& Garza, G. (2015). Mending fences: Defining the domains and approaches of quantitative and qualitative research. Qualitative Psychology, 2(2), 199-209. doi: 10.1037/qup0000030

Levac, J., Toal-Sullivan, D., \& O’Sullivan, T. L. (2012). Household emergency preparedness: A literature review. Journal of Community Health, 37, 725-733. doi: 10.1007/s10900011-9488-X

Lindell, M. K. (2013). Disaster studies. Current Sociology Review, 61, 797-825. doi: $10.1177 / 0011392113484456$

Lindell, M. K., Arlikatti, S., \& Prater, C. S. (2009). Why people do what they do to protect against earthquake risk: Perceptions of hazard adjustment attributes. Risk Analysis, 29(8), 1072-1088. doi: 10.1111/j.1539-6924. 2009.01243.x

Lindell, M. K., \& Hwang, S. N. (2008). Households' perceived personal risk and responses in a multihazard environment. Risk Analysis, 28(2), 539-556. doi: 10.1111/j.15396924.2008.01032.x

Lindell, M. K., \& Perry, R. W. (2000). Household adjustment to earthquake hazard: A review of research. Environment and Behavior, 32, 590-630. doi: 10.1177/001391600219726 21

Lindell, M. K., \& Perry, R. W. (2012). The Protective Action Decision Model: Theoretical modifications and additional evidence. Risk Analysis, 32, 616-632. doi: 10.1111/j.15396924.2011.01647.x

Lindell, M. K., \& Whitney, D. J. (2000). Correlates of seismic hazard adjustment adoption. Risk Analysis, 20, 13-25. doi: 10.1111/0272-4332.00002 
Lingler, J. H., Schmidt, K., Gentry, A., Hu, L., \& Terhorst, L. (2015). Perceived Research Burden Assessment (PeRBA): Instrument development and psychometric evaluation. Journal of Empirical Research on Human Research Ethics, 9(4), 46-49. doi: $10.1177 / 1556264614545037$

Liu, Y., Ouyang, Z., \& Cheng, P. (2019). Predicting consumers' adoption of electric vehicles during the city smog crisis: An application of the Protective Action Decision Model. Journal of Environmental Psychology, 64, 30-38. doi: 10.1016/j.envp.2019.04.013

Locke, E. A., Shaw, K. N., Saari, L. M., \& Latham, G. P. (1981). Goal setting and task performance: 1969-1980. Psychological Bulletin, 90, 125-152. doi: 10.1037/00332909.90.1.125

Lonsdale, C., Hodge, K., \& Rose, E. A. (2006). Pixels vs. paper: Comparing online and traditional survey methods in sport psychology. Journal of Sport and Exercise Psychology, 28, 100-108.

Luszczynska, A., Schwarzer, R., Lippke, S. \& Mazurkiewicz, M. (2011). Self-efficacy as a moderator of the planning-behaviour relationship in interventions designed to promote physical activity. Psychology \& Health, 26, 151-166. doi: 10.1080/08870446.2011.53 1571

MacDonald, E., Johnson, V., Gillies, M., \& Johnston, D. (2017). The impact of a museumbased hazard education program on students, teachers and parents. International Journal of Disaster Risk Reduction, 21, 360-366. doi: 10.1016/j.ijdrr.2017.01.010

MacQueen, K. M., McLellan, E., Metzger, D. S., Kegeles, S., Strauss, R. P., Scotti, R., ... Trotter, R. T. (2001). What is community? An evidence-based definition for participatory public health. American Journal of Public Health, 91, 1929-1938. doi: 10.2105/ajph.91.12.1929

Magill, C., \& Blong, R. (2005). Volcanic risk ranking for Auckland, New Zealand. II: Hazard consequences and risk calculation. Bulletin of Volcanology, 67, 340-349. doi: $10.1007 / \mathrm{s} 00445-004-0375-5$

Mamula-Seadon, L. \& McLean, I. (2015). Response and early recovery following 4 September 2010 and 22 February 2011 Canterbury earthquakes: Societal resilience and the role of governance. International Journal of Disaster Risk Reduction, 14, 82-95. doi: 10.1016/j.ijdrr.2015.01.005

Manning, M., \& Bettencourt, B. A. (2011). Depression and medication adherence among breast cancer survivors: Bridging the gap with the theory of planned behaviour. Psychology \& Health, 26, 1173-1187. doi: 10.1080/08870446.2010.542815 
Maynor, L., \& Arbon, P. (2015). Defining disaster: The need for harmonisation of terminology. Australasian Journal of Disaster and Trauma Studies, 19, 21-25.

McClure, J., Allen, M.W., \& Walkey, F. (2001). Countering fatalism: Causal information in news reports affects judgments about earthquake damage. Basic and Applied Social Psychology, 23, 109-121. doi: 10.1207/S15324834BASP2302_3

McClure, J., Doyle, E.E.H., \& Velluppillai, J.M. (2015). A tale of two cities: Judgments about earthquake and aftershock probabilities across time windows. International Journal of Disaster Risk Reduction, 14, 15-26. doi: 10.1016/j.ijdrr.2014.11.010

McClure, J., Ferrick, M., Henrich, L., \& Johnston, D. (2019). Risk judgments and social norms: Do they relate to preparedness after the Kaikōura earthquakes? Australasian Journal of Disaster and Trauma Studies, 23(2), 41-51.

McClure, J., Henrich, L., Johnston, D., \& Doyle, E.E.H. (2016). Are two earthquakes better than one? How earthquakes in two different regions affect risk judgments and preparation in three locations. International Journal of Disaster Risk Reduction, 16, 192-199. doi: 10.1016/j.ijdrr.2016.03.003

McClure, J., Johnston, D., Henrich, L., Milfont, T.L., \& Becker, J. (2015). When a hazard occurs where it is not expected: Risk judgments about different regions after the Christchurch earthquakes. Natural Hazards, 75(1), 635-652. doi: 10.1007/s11069-014$1338-6$

McClure, J., \& Sibley, C.G. (2011). Framing effects on disaster preparation: Is negative framing more effective? Australasian Journal of Disaster and Trauma Studies, 2011(1).

McClure, J., Spittal, M. J., Fischer, R., \& Charleson, A. (2015). Why do people take fewer damage mitigation actions than survival actions? Other factors outweigh cost. Natural Hazards Review, 16(2), 1-8. doi: 10.1061/(ASCE)NH.1527-6996.0000152

McClure, J., Sutton, R. M., \& Sibley, C. G. (2007). Listening to reporters or engineers? How instance-based messages about building design affect earthquake fatalism. Journal of Applied Social Psychology, 37, 1956-1973. doi: 10.1111/j.1559-1816.2007.00245.x

McClure, J., \& Velluppillai, J. (2013). The effects of news media reports on earthquake attributions and preventability judgments: Mixed messages about the Canterbury earthquake. Australasian Journal of Disaster and Trauma Studies, 2013(1), 27-36.

McClure, J., White, J., \& Sibley, C.G. (2009). Framing effects on preparation intentions: Distinguishing actions and outcomes. Disaster Prevention and Management, 18(2), 187-199. doi: 10.1108/09653560910953252 
McClure, J., Wills, C., Johnston, D., \& Recker, C. (2011). How the 2010 Canterbury (Darfield) earthquake affected earthquake risk perception: Comparing citizens inside and outside the earthquake region. Australasian Journal of Disaster and Trauma Studies, 2011(1), $3-10$.

McEachan, R. R. C., Conner, M., Taylor, N. J., \& Lawton, R. J. (2011). Prospective prediction of health-related behaviours with the Theory of Planned Behaviour: A meta-analysis. Health Psychology Review, 5(2), 97-144. doi: 10.1080/17437199.2010.521684

McDermott, M.S., Oliver, M., Simnadis, T., Beck, E. J., Coltman, T., Iverson, D., ... Sharma, R. (2015). The theory of planned behaviour and dietary patterns: A systematic review and meta-analysis. Preventive Medicine, 81, 150-156. doi: 10.1016/j.ypmed.2015.08. 020

McIvor, D., \& Paton, D. (2007). Preparing for natural hazards: Normative and attitudinal influences. Disaster Prevention and Management, 16, 79-88. doi: 10.1108/09653560 710729839

McKenzie-Mohr, D. (2011). Fostering sustainable behavior: An introduction to community based social marketing ( $3^{\text {rd }}$ ed.). Canada: New Society Publishers.

McMillan, B., Conner, M., Green, J., Dyson, L., Renfrew, M., \& Woolridge, M. (2009). Using an extended Theory of Planned Behaviour to inform interventions aimed at increasing breastfeeding uptake in primiparas experiencing material deprivation. British Journal of Health Psychology, 14, 379-403. doi: 10.1348/135910708X336112

McLennan, J., Marques, M., \& Every, D. (2020). Conceptualising and measuring psychological preparedness for disaster: The Psychological Preparedness for Disaster Threat Scale. Natural Hazards, 11 p. doi: 10.1007/s11069-020-03866-4

McRae, C., McClure, J., Henrich, L., Leah, C., \& Charleson, A. (2017). Reactions to earthquake hazard: Strengthening commercial buildings and voluntary earthquake safety checks on houses in Wellington, New Zealand. International Journal of Disaster Risk Reduction, 28, 465-474. doi: 10.1016/j.ijdrr.2017.12.007

McSaveney, E. (2017). Historic earthquakes: Earthquakes in Māori tradition. Retrieved from https://teara.govt.nz/en/historic-earthquakes/page-1

Michie, S., Ashford, S., Sniehotta, F. F., Dombrowski, S. U., Bishop, A., \& French, D. P. (2011). A refined taxonomy of behaviour change techniques to help people change their physical activity and healthy eating behaviours: The CALO-RE taxonomy. Psychology \& Health, 26, 1479-1498. doi: 10.1080/08870446.2010.540664 
Michie, S., Johnston, M., Francis, J., Hardeman, W., \& Eccles, M. (2008). From theory to intervention: Mapping theoretically derived behavioural determinants to behaviour change techniques. Applied Psychology: An International Review, 57, 660-680. doi: 10.1111/j.1464-0597.2008.00341.x

Milfont, T. L. (2009). The effects of social desirability on self-reported environmental attitudes and ecological behaviour. Environmentalist, 29, 263-269. doi: 10.1007/s10669-0089192-2

Milfont, T. L., \& Fischer, R. (2010). Testing measurement invariance across groups: Applications in cross-cultural research. International Journal of Psychological Research, 3, 112-131.

Ministry for Primary Industries. (2014). Government assistance for climatic events and natural disasters impacting on-farm. Retrieved from mpi.govt.nz/dmsdocument/3629

Morrison, D., Lawrence, C., \& Oehmen, R. (2014). Community level influence on individual behaviours. Bushfire CRC: Australia.

Mortensen, C. R., Neel, R., Cialdini, R. B., Jaeger, C. M., Jacobson, R. P., \& Ringel, M. M. (2017). Trending norms: A lever for encouraging behaviors performed by the minority. Social Psychological and Personality Science, 10(2), 201-210. doi: $10.1177 / 1948550617734615$

Mulilis, J.-P., \& Duval, T. S. (1995). Negative threat appeals and earthquake preparedness: A Person-Relative-to-Event model of coping with threat. Journal of Applied Social Psychology, 25, 1319-1339. doi: 10.1111/j.1559-1816.1995.tb02620.x

Mulilis, J.-P., \& Duval, T. S. (1997). The PrE model of coping and tornado preparedness: Moderating effects of responsibility. Journal of Applied Social Psychology, 27, 17501766. doi: 10.1111/j.1559-1816.1997.tb01623.x

Mulilis, J., Duval, T. S., \& Lippa, R. (1990). The effects of a large destructive local earthquake on earthquake preparedness as assessed by an Earthquake Preparedness Scale. Natural Hazards, 3, 357-371. doi: 10.1007/BF00124393

Muthén, L. K., \& Muthén, B. O. (1998-2017). Mplus User's Guide. Eighth Edition. Los Angeles, CA: Muthén \& Muthén.

Najafi, M., Ardalan, A., Akbarisari, A., Noorbala, A. A., \& Elmi, H. (2017). The theory of planned behaviour and disaster preparedness. PLoS Currents Disasters. doi: 10.1371/currents.dis.4da18e0f1479bf6c0a94b29e0dbf4a72 
Najafi, M., Khankeh, H. R., Elmi, H., \& Pourvakhshoori, N. (2018). Behavioral, normative and control beliefs about earthquake preparedness: A deductive content analysis study. PLoS Currents, 10, 17 p. doi: 10.1371/currents.dis.20fbad29d53fb164ac2699dc2736d 804

National Emergency Management Agency. (2019). National Disaster Resilience Strategy. New Zealand: New Zealand Government.

Nojang, E. N., \& Jensen, J. (2020). Conceptualizing individual and household disaster preparedness: The Perspective from Cameroon. International Journal of Disaster Risk Science, 11, 333-346. doi: 10.1007/s13753-020-00258-x

Nolan, J. M., Schultz, P. W., Cialdini, R. B., Goldstein, N. J., \& Griskevicius, V. (2008). Normative social influence is underdetected. Personality and Social Psychology Bulletin, 34(7), 913-923. doi: 10.1177/0146467208316691

Norman, P. Conner, M., \& Bell, R. (2000). The Theory of Planned Behaviour and exercise: Evidence for the moderating role of past behaviour. British Journal of Health Psychology, 5, 249-261. doi: 10.1348/135910700168892

O’Connor, F., M., Johnston, D., \& Evans, I. M. (2011). The context in which we examine disasters in New Zealand: An editorial. New Zealand Journal of Psychology, 40(4), 26.

Ohtani, K. (1984). A note of the Wald, $L R$ and $L M$ tests and misspecification. Economics Letters, 14, 31-35. doi: 10.1016/0165-1765(84)90024-7

Orchiston, C., Manuel, C., Coomer, M., Becker, J., \& Johnston, D. (2013). The 2009 New Zealand West Coast ShakeOut: Improving earthquake preparedness in a region of high seismic risk. Australasian Journal of Disaster and Trauma Studies, 2013(2), 55-61.

Ozaki, T., \& Nakayachi, K. (2015). Effects of descriptive norms and mutual relationships on precautionary behavior toward earthquakes. Japanese Journal of Social Psychology, 30(3), 175-182. doi: 10.14966/jssp.30.3_175

Parent, N., \& Fortin, F. (2000). A randomized, controlled trial of vicarious experience through peer support for male first-time cardiac surgery patients: Impact on anxiety, selfefficacy expectation, and self-reported activity. The Journal of Cardiopulmonary and Acute Care, 29, 369-400. doi: 10.1067/mhl.2000.110626.

Park, H. S., \& Smith, S. W. (2007). Distinctiveness and influence of subjective norms, personal descriptive and injunctive norms, and societal descriptive and injunctive norms on behavioural intent: A case of two behaviors critical to organ donation. Human Communication Research, 33, 194-218. 
Paton, D. (2013). Disaster resilient communities: Developing and testing an all-hazards theory. Journal of Integrated Disaster Risk Management, 3, 1-17. doi: 10.5595/idrim.2013. 0050

Paton, D. (2019). Disaster risk reduction: Psychological perspectives on preparedness. Australian Journal of Psychology, 71, 327-341. doi: 10.1111/ajpy.12237

Paton, D., Anderson, E., Becker, J., \& Peterson, J. (2015). Developing a comprehensive model of hazard preparedness: Lessons from the Christchurch earthquake. International Journal of Disaster Risk Reduction, 14(1), 37-45. doi: 10.1016/j.ijdrr.2014.11.011

Paton, D., \& Buergelt, P. (2019). Risk, transformation and adaptation: Ideas for reframing approaches to disaster risk reduction. International Journal of Environmental Research and Public Health, 16, 2594-2612. doi: 10.3390/ijerph16142594

Paton, D. \& Johnston, D. (2001). Disasters and communities: Vulnerabilities, resilience, and preparedness. Disaster Prevention and Management, 10(4), 270-277. doi: 10.1108/EUM0000000005930

Paton, D., Johnston, D., Smith, L., \& Millar, M. (2001). Responding to hazard effects: Promoting resilience and adjustment adoption. Australian Journal of Emergency Management, 16(1), 47-52.

Paton, D., Kershoult, H., \& Skinner, I. (2017) Hazard readiness and resilience. In D. Paton and D.M. Johnston (Eds). Disaster Resilience: An integrated approach $\left(2^{\text {nd }}\right.$ Ed). (pp. 236254) Springfield, IL, Charles C. Thomas.

Paton, D., Millar, M., \& Johnston, D. (2001). Community resilience to volcanic hazard consequences. Natural Hazards, 24, 157-169.

Paton, D., Okada, N., \& Sagala, S. (2013). Understanding preparedness for natural hazards: A cross cultural comparison. Journal of Integrated Disaster Risk Management, 3, 18-35.

Paton, D., Sagala, S., Okada, N., Jang, L-J., Buergelt, P., \& Gregg, C. E. (2010). Making sense of natural hazard mitigation: Personal, social and cultural influences. Environmental Hazards, 9, 183-196. doi: 10.3763/ehaz.2010.0039

Paton, D., Smith, L., \& Johnston, D. M. (2000). Volcanic hazards: Risk perception and preparedness. New Zealand Journal of Psychology, 29(2), 86-91.

Paton, D., Smith, L., \& Johnston, D. (2005). When good intentions turn bad: Promoting natural hazard preparedness. Australian Journal of Emergency Management, 20, 25-30.

Perry, R. W. (2004). Disaster exercise outcomes for professional emergency personnel and citizen volunteers. Journal of Contingencies and Crisis Management, 12, 64-75. doi: 10.1111/j.0966-0879.2004.00436.x 
Perry. R. W. (2018). Defining disaster: An evolving concept. In H. Rodriguez, W. Donner, \& J. Trainor (Eds.), Handbook of disaster research. (2 ${ }^{\text {nd }}$ ed., pp. 3-22). Switzerland: Springer. doi: 10.1007/978-3-319-63254-4_1

Perry, S., Cox, D., Jones, L., Bernknopf, R., Goltz, J., Hudnut, K., ... Wein, A. (2008). The ShakeOut earthquake scenario: A story that Southern Californians are writing. U.S. Geological Survey: Virginia, US.

Perry, R. W., \& Lindell, M. K. (2008). Volcanic risk perception and adjustment in a multihazard environment. Journal of Volcanology and Geothermal Research, 172(3-4), 170178. doi: 10.1016/j.volgeores.2007.12.006

Peters, G.-J. Y., Ruiter, R. A. C., \& Kok, G. (2012). Threatening communication: A critical reanalysis and a revised meta-analytic test of fear appeal theory. Health Psychology Review, 7(Supplement 1), 8-31. doi: 10.1080/174

Peters, R., M., \& Templin, T. N. (2010). Theory of Planned Behavior, self-care motivation, and blood pressure self-care. Research and Theory for Nursing Practice, 24(3), 172186. doi: 10.1891/1541-6577.24.3.172

Pihama, L., Southey, K., \& Tiakiwai, S. (Eds.). (2015). Kaupapa rangahau: A reader. A collection of readings from the Kaupapa Rangahau workshops series (2nd ed.). Hamilton, New Zealand: Te Kotahi Research Institute.

Potter, S. H., Becker, J. S., Johnston, D. M., \& Rossiter, K. P. (2015). An overview of the impacts of the 2010-2011 Canterbury earthquakes. International Journal of Disaster and Trauma Studies, 14, 6-14. doi: 10.1016/j.ijdrr.2015.01.014

Power, W., Wallace, L., \& Reyners, M. (2008). Tsunami hazard posed by earthquakes on the Hikurangi subduction zone interface. GNS Science Report 2008(40). 44 p.

Putnick, D. L., \& Bornstein, M. H. (2016). Measurement invariance conventions and reporting: The state of the art and future directions for psychological research. Developmental Review, 41, 71-90. doi: 10.1016/j.dr.2016.06.004

Quarantelli, E. L. (1980). The study of disaster movies: Research findings, problems and implications. University of Delaware Disaster Research Center Preliminary paper, 64, $30 \mathrm{p}$.

Ramirez, M., Kubicek, K., Peek-Asa, C., \& Wong, M. (2009). Accountability and assessment of emergency drill performance at schools. Family \& Community Health, 32, 105-114. doi: 10.1097/FCH.0b013e3181994662 
Ras, M. (2017, May 18). Natural disasters don't exist but natural hazards do. Retrieved from www.undp.org/content/undp/en/home/blog/2017/5/18/Natural-disasters-don-t-existbut-natural-hazards-do.html

Rhodes, R. E., \& Dickau, L. (2012). Experimental evidence for the intention-behavior relationship in the physical activity domain: A meta-analysis. Health Psychology, 31, 724-727. doi: 10.1037/a0027290

Richetin, J., Conner, M., \& Perugini, M. (2011). Not doing is not the opposite of doing: Implications for attitudinal models of behavioral predictions. Personality and Social Psychology Bulletin, 37, 40-54. doi: 10.1177/0146167210390522

Riebl, S. K., Estabrooks, P. A., Dunsmore, J. C., Savla, J., Frisard, M. I. Dietrick, A. M., ... Davy, B. M. (2015). A systematic literature review and meta-analysis: The theory of planned behavior's application to understand predict nutrition-related behaviors in youth. Eating Behaviors, 18, 160-178. doi: 10.1016/j.eatbeh.2015.05.016

Rimal, R. N., \& Lapinski, M. K. (2015). A re-explication of social norms, ten years later. Communication Theory, 25(4), 393-409. doi: 10.1111/comt.12080

Rivis, A., Sheeran, P., \& Armitage, C. J. (2009). Expanding the affective and normative components of the Theory of Planned Behavior: A meta-analysis of anticipated affect and moral norms. Journal of Applied Social Psychology, 39, 2985-3019. doi: 10.1111/j.1559-1816.2009.00558.x

Rochford, Jr., E. B., \& Blocker, T. J. (1991). Coping with "natural" hazards as stressors: the predictors of activism in a flood disaster. Environment and Behavior, 23(2), 171-194. doi: $10.1177 / 001391$

Ronan, K. R., Crellin, K., Johnston, D. M., Finnis, K., Paton, D., \& Becker, J. C. (2008). Promoting child and family resilience to disasters: Effects, interventions, and prevention effectiveness. Children, Youth and Environments, 18(1), 332-353.

Rowney, C., Farvid, P., \& Sibley, C. G. (2014). "I laugh and say I have 'Earthquake Brain!'”: Resident responses to the September 2010 Christchurch earthquake. New Zealand Journal of Psychology, 43(2), 4-13

Russell, L. A., Goltz, J. D., \& Bourque, L. B. (1995). Preparedness and hazard mitigation actions before and after two earthquakes. Environment and Behavior, 27, 744-770. doi: $10.1177 / 0013916595276002$

Rustemli, A. \& Karanci, N. (1999). Correlates of earthquake cognitions and preparedness behavior in a victimized population. The Journal of Social Psychology, 139, 91-101. doi: 10.1080/00224549909598364 
Sachdeva, S. (2016, November 25). Opinion: Our response to the latest earthquake is worrying - are we ready for the next 'big one'? Stuff. Retrieved from www.stuff.co.nz

Sagala, S., Okada, N., \& Paton, D. (2009). Predictors of intention to prepare for volcanic risks in Mt Merapi, Indonesia. Journal of Pacific Rim Psychology, 3, 47-54.

Sale, J. E. M., Lohfeld, L. H., Brazil, K. (2002). Revisiting the quantitative-qualitative debate: Implications for mixed-methods research. Quality \& Quantity, 36, 43-53. doi: 10.1023/A:1014301607592

Sanquini, A. M., Thapaliya, S. M., \& Wood, M. M. (2016). A communications intervention to motivate disaster risk reduction. Disaster Prevention and Management, 25, 245-259. doi: 10.1108/DPM-11-2015-0256

Satherley, N., Milojev, P., Greaves, L. M., Huang, Y., Osborne, D., Bulbulia, J., \& Sibley, C. S. (2015). Demographic and psychological predictors of panel attrition: Evidence from the New Zealand Attitudes and Values Study. PLoSONE, 10(3), e0121950. doi: 10.1371/journal.pone.0121950

Schröder, N. (2010). Framing disaster: Images of nature, media, and representational strategies in Hollywood disaster movies. In L. Volkmann, N. Grimm, I. Detmers, \& K. Thomson (Eds.), Local natures, global responsibilities (pp 289-306). Leiden, The Netherlands: Brill | Rodopi.

Shadwell, T. (2016, September 16). Wellington's Civil Defence Centres reformed into 'Community Emergency Hubs', which won't stock survival supplies. The Dominion Post. Retrieved from www.stuff.co.nz

Sharp, E. C., Pelletier, L. G., \& Lévesque, C. (2006). The double-edged sword of rewards for participation in psychology experiments. Canadian Journal of Behavioural Science, 38(3), 269-277. doi: 10.1037/cjbs2006014

Sheeran, P., Harris, P. R., \& Epton, T. (2014). Does heightening risk appraisals change people's intentions and behavior? A meta-analysis of experimental studies. Psychological Bulletin, 140(2), 511-543. doi: 10.1037/a0033065

Singer, E., \& Couper, M. P. (2008). Do incentives exert undue influence on survey participation? Experimental evidence. Journal of Empirical Research on Human Research Ethics, 3(3), 49-56. doi: 10.1525/jer.2008.3.3.49

Smith, N. (2015, May 10). More targeted approach to earthquake-prone buildings. Retrieved from http://www.beehive.govt.nz/release/more-targeted-approach-earthquake-pronebuildings 
Smith, J. R., Louis, W. R., Terry, D. J., Greenaway, K. H., Clarke, M. R., \& Cheng, X. (2012). Congruent or conflicted? The impact of injunctive and descriptive norms on environmental intentions. Journal of Environmental Psychology, 32, 353-361. doi: 10.1016/j.envp.2012.06.001

Sniehotta, F.F. (2009). Towards a theory of intentional behaviour change: Plans, planning, and self-regulation. British Journal of Health Psychology, 14, 261-273. doi: $10.1348 / 135910708 X 389042$

Sniehotta, F. F., Scholz, U., \& Schwarzer, R. (2005). Bridging the intention-behaviour gap: Planning, self-efficacy, and action control in the adoption and maintenance of physical exercise. Psychology and Health, 20, 143-160. doi: 10.1080/08870440512331317670

Solberg, C., Rossetto, T., \& Joffe, H. (2010). The social psychology of seismic hazard adjustment: re-evaluating the international literature. Natural Hazards and Earth System Sciences. 10, 1663-1677. doi: 10.5194/nhess-10-1663-2010, 2010

Sparkman, G. \& Walton, G. M. (2017). Dynamic norms promote sustainable behavior, even if it is counternormative. Psychological Science, 28, 1663-1674. doi: 10.1177/095679761 7719950

Spittal, M. J., McClure, J., Siegert, R. J., \& Walkey, F. H. (2005). Optimistic bias in relation to preparedness for earthquakes. Australasian Journal of Disaster and Trauma Studies, 2005(1). Retrieved from www.massey.ac.nz/ trauma/issues/2005-1/spittal.htm

Spittal, M. J., McClure, J., Siegert, R. J., \& Walkey, F. H. (2008). Predictors of two types of earthquake preparation: Survival activities and mitigation activities. Environmental Behaviour, 40(6), 798-817. doi: 10.1177/0013916507309864

Spittal, M. J., Walkey, F. H., McClure, J., Siegert, R. J., \& Ballantyne, K. (2006). The Earthquake Readiness Scale: The development of a valid and reliable unifactorial measure. Natural Hazards, 39, 15-29. doi: 10.1007/s11069-005-2369-9

Stats NZ. (2002). Census of population and dwellings, 2001. Retrieved from http://archive.stats.govt.nz/Census/2001-Census-data/population-and-dwellingstatistics.aspx

Stevenson, J. R., Becker, J., Cradock-Henry, N., Johal, S., Johnston, D., Orchiston, C., \& Seville, E. (2017). Economic and social reconnaissance: Kaikōura earthquake 2016. Bulletin of the New Zealand Society for Earthquake Engineering, 50, 343-351. 
Sun, G., Acheampong, R. A., Lui, H., \& Pun, V. C. (2015). Understanding walking behaviour among university students using Theory of Planned Behavior. International Journal of Environmental Research and Public Health, 12, 13794-13806. doi: 10.3390/ijerph121113794

Tanes, Z., \& Cho, H. (2013). Goal setting outcomes: Examining the role of goal interaction in influencing the experience and learning outcomes of video game play for earthquake preparedness. Computers in Human Behavior, 29, 858-869. doi: 10.1016/j.chb.2012. 11.003

Tang, J.-S., \& Feng, J.-Y. (2018). Residents' disaster preparedness after the Meinong Taiwan earthquake: A test of Protection Motivation Theory. International Journal of Environmental Research and Public Health, 15, 1-12. doi: 10.3390/ijerph15071434

Tanner Jr., J. F., Day, E., \& Crask, M. R. (1989). Protection Motivation Theory: An extension of Fear Appeals Theory in communication. Journal of Business Research, 19, 267-276. doi: 10.1016/0148-2963(89)9008-8

Teo, T. (2013). Online and paper-based survey data: Are they equivalent? British Journal of Educational Technology, 44(6), e196-198. doi: 10.1111/bjet.12074

Terpstra, T., \& Lindell, M. K. (2012). Citizens' perceptions of flood hazard adjustments: An application of the Protective Action Decision Model. Environment and Behavior, 45(8), 993-1018. doi: 10.1177/0013916512452427

Tipler, K. S., Tarrant, R. A., Johnston, D. M., \& Tuffin, K. F. (2016). New Zealand ShakeOut exercise: Lessons learned by schools. Disaster Prevention and Management, 25(4), 550-563. doi: 10.1108/DPM-01-2016-0018

Tippett, M. K. (2018). Extreme weather and climate. Climate and Atmospheric Science, 1, 12. doi: 10/1038/s41612-018-0057-1

Topa, G., \& Moriano, J. A. (2010). Theory of planned behavior and smoking: Meta-analysis and SEM model. Substance Abuse and Rehabilitation, 1, 23-33. doi: 10.2147/SAR.S15168

Tsorbatzoudis, H. (2005). Evaluation of a planned behavior theory-based intervention programme to promote healthy eating. Perceptual and Motor Skills, 101, 587-604. doi: 10.2466/pms.101.2.587-604

Tuohy, R., \& Stephens, C. (2016). Older adults' meanings of preparedness: A New Zealand perspective. Ageing and Society, 36, 613-630. doi: 10.1017/S0144686X14001408

Tversky, A., \& Kahneman, D. (1974). Judgment under uncertainty: Heuristics and biases. Science, 185(4157), 1124-1131. doi: 10.1126/science.185.4157.1124 
Tyson, M., Covey, J., \& Rosenthal, H. E. S. (2014). Theory of planned behavior interventions for reducing heterosexual risk behaviors: A meta-analysis. Health Psychology, 33, 1454-1467. doi: 10.1037/hea0000047

UNDRR. (2015). Sendai Framework for Disaster Risk Reduction. Geneva, Switzerland: United Nations.

United Nations General Assembly. (2016). Report of the open-ended intergovernmental expert working group on indicators and terminology relating to disaster risk reduction [PDF file]. Retrieved from www.preventionweb.net/publications/view/51748

Vallance, S. (2015). Disaster recovery as participation: Lessons from the Shaky Isles. Natural Hazards, 75, 1287-1301. doi: 10.1007/s11069-014-1361-7

van de Mortel, T. F. (2008). Faking it: Social desirability response bias in self-report research. Australian Journal of Advanced Nursing, 25(4), 40-48.

Vandenberg, R. J., \& Lance, C. E. (2000). A review and synthesis of the measurement invariance literature: Suggestions, practices, and recommendations for organizational research. Organizational Research Methods, 3(1), 4-70. doi: 10.1177/1094428100310 02

Vinnell, L. J. (2016). Examining the effects of message framing and social norms on judgments of earthquake legislation (Master's thesis). Retrieved from ResearchArchive. (5471)

Vinnell, L. J., McClure, J., \& Milfont, T. L. (2017). Do framing messages increase support for earthquake legislation? Disaster Prevention and Management, 26, 28-40. doi: 10.1108/ DPM-06-2016-0127

Vinnell, L. J., Milfont, T. L., \& McClure, J. (2018). Do social norms affect support for earthquake strengthening legislation? Comparing the effects of descriptive and injunctive norms. Environment and Behavior, 51, 376-400. doi: 10.1177/00139165177 52435

Vinnell, L. J., Milfont, T. L., \& McClure, J. (2019). The impact of the Kaikōura earthquake on risk-related behaviour, perceptions, and social norm messages. Australasian Journal of Disaster and Trauma Studies, 23(2), 53-64.

Vinnell, L.J., Orchiston, C., Becker, J., \& Johnston, D. (2019). Pathways to earthquake resilience: Learning from past events. Australasian Journal of Disaster and Trauma Studies, 23(2), 35-40. 
Vinnell, L. J., Wallis, A., Becker, J. S., \& Johnston, D. M. (2020). Evaluating the ShakeOut drill in Aotearoa/New Zealand: Effects on knowledge, attitudes, and behaviour. International Journal of Disaster Risk Reduction, 48, 1-9. doi: 10.1016/j.ijdrr.2020.101721

von Davier, A. A. (2003). Large sample tests for comparing regression coefficients in models with normally distributed variables. Educational Teaching Services: Princeton, NJ.

Walker, G. J., Courneya, K. S., \& Deng, J. (2006). Ethnicity, gender, and the Theory of Planned Behavior: The case of playing the lottery. Journal of Leisure Research, 38, 224-248. doi: 10.1080/00222216.2006.11950077

Wallemacq, P., \& House, R. (2018). Economic losses, poverty \& disasters: 1998-2017. United Nations Office for Disaster Risk Reduction.

Wan, C., Shen, G. Q., \& Choi, S. (2017). Experiential and instrumental attitudes: Interaction effect of attitude and subjective norm on recycling intention. Journal of Environmental Psychology, 50, 69-79. doi: 10.1016/j.jenvp.2017.02.006

Webb, T. L., \& Sheeran, P. (2006). Does changing behavioral intentions engender behaviour change? A meta-analysis of the experimental evidence. Psychological Bulletin, 132, 249-268. doi: 10.1037/0033-2909.132.2.249

Wellington City Council. (2017). Wellington Resilience Strategy [PDF file]. Retrieved from www.wellington.govt.nz/ /media/about-wellington/resilientwellington/files/strategy/resilience-strategyj001767-100-web-pdf?la=en

Wellington Region Emergency Management Office. (n.d.). Earthquake planning guide [PDF file]. Retrieved from https://wremo.nz/assets/Publications/Earthquake-PlanningGuide.pdf

Westcott, R., Ronan, K., Bambrick, H., \& Taylor, M. (2017). Expanding Protection Motivation Theory: Investigating an application to animal owners and emergency responders in bushfire emergencies. BMC Psychology, 5, 1-14. doi: 10.1186/s40359-017-0182-3

White, K. M., Smith, J. R., Terry, D. J., Greenslade, J. H., \& McKimmie, B. M. (2009). Social influence in the Theory of Planned Behaviour: The role of descriptive, injunctive, and in-group norms. British Journal of Social Psychology, 48, 135-158. doi: 10.1348/014466608X295207

Williams, S. L. \& French, D. P. (2011). What are the most effective intervention techniques for changing physical activity self-efficacy and physical activity behaviour - and are they the same? Health Education Research, 26(2). 308-322. doi: 10.1093/her/cyr005 
Wilson, T. M., \& Cole, J. W. (2007). Potential impact of ash eruptions on dairy farms from a study of the effects on a farm in eastern Bay of Plenty, New Zealand: Implications for hazard mitigation. Natural Hazards, 43, 103-128. doi: 10.1007/s11069-007-9111-8

Witvorapong, N., Muttarak, R., \& Pothisiri, W. (2015). Social participation and disaster risk reduction behaviours in tsunami prone areas. PLoSONE, 10(7): e0130862, 1-20. doi: 10.1371/journal.pone.0130862

Wolf, E. J., Harrington, K. M., Clark, S. L., \& Miller, M. W. (2013). Sample size requirements for structural equation models: an evaluation of power, bias, and solution propriety. Educational and Psychological Measurement, 76, 913-934. doi: 10.1177/001316441 3495237

Yang, D. (2013). The communication effects of audience situation and message framing on smoking cessation. International Journal of Management, Economics and Social Sciences, 2(4), 252-264.

Yang, Z. J. (2014). Predicting young adults' intentions to get the H1N1 vaccine: An integrated model. Journal of Health Communication, 20, 67-79. doi: 10.1080/10810730.2014.90 4023.

Zhu, Y., Zhang, Z., Ling, Y., \& Wan, H. (2017). Impact of intervention on breastfeeding outcomes and determinants based on Theory of Planned Behavior. Women and Birth, 30, 146-152. doi: 10.1016/j.wombi.2016.09.011 
Appendix 3.A: Study 1 information sheet

Information Sheet: Understanding why people in Wellington prepare for natural disasters Ethics Application ID number: 0000025441

What is the purpose of this research?

- This research will help us to understand why people in Wellington do and do not prepare for natural disasters, including their beliefs about whether it's a common and approved behaviour.

Who is conducting the research?

- We are a team of researchers in the School of Psychology at Victoria University of Wellington. Dr Taciano Milfont and A.Prof Ron Fischer are the primary supervisors of this project. Prof John McClure and Dr Wokje Abrahamse are secondary supervisors. This research has been approved by the School of Psychology Human Ethics Committee under delegated authority of Victoria University of Wellington's Human Ethics Committee.

What is involved if you agree to participate?

- If you agree to participate in this study, you will complete a short survey where you will respond to a range of different questions such as "Where you live, how likely is it that a natural disaster will occur in the following five years?" as well as letting us know what preparations you've made, and which ones you intend to do. - Some questions will ask you about your previous or potential future experiences of natural disasters which might cause emotional discomfort. You do not have to answer these questions if you are concerned about how they will make you feel.

- We will also ask you for some demographic information e.g., age, income, ethnicity. This is so that we can have a deeper understanding of the additional factors that may impact on natural disaster preparation.

- We anticipate that the survey will take you no more than 15 to 20 minutes to complete.

- During the research you are free to withdraw at any point before your survey has been completed.

- As a token of appreciation you will be given the opportunity to go into the draw for one of three $\$ 100$ supermarket gift cards.

Privacy and Confidentiality

- This survey is completely confidential. We want to make your responses unidentifiable so please do not enter any identifying information in the text boxes.

- We will keep your survey responses for five years after publication at which point they will then be destroyed.

- In accordance with the requirements of some scientific journals and organizations, the information from your survey that does not identify you may be shared with other competent researchers.

- An electronic version of the data will remain indefinitely in the custody of the researchers at Victoria University of Wellington.

- At the end of the survey, you will have the option to provide your email address to enter the prize draw. This information will be kept separate from the survey data so that your responses cannot be linked to you. Email addresses will be held securely on a passwordprotected database and destroyed immediately after prizes have been allocated (approximately 2 weeks after the survey closes).

What happens to the information that you provide? 
- The overall findings will form part of two PhD theses and/or be published in scientific journals.

- If you would like to know the results of this study, they will be available approximately August, 2018 through A. Prof Ronald Fischer's research lab website (mindcultureevolution.com).

If you have further questions or concerns you would like answered before taking part in the survey, you can contact the researchers at the email addresses below.

Thank you for considering participation in this research.

Amanda Wallis

Victoria University of Wellington

amanda.wallis@vuw.ac.nz

A. Prof Ronald Fischer Victoria University of Wellington ronald.fischer@vuw.ac.nz

Dr Wokje Abrahamse

Victoria University of Wellington wokje.abrahamse@vuw.ac.nz
Lauren Vinnell

Victoria University of Wellington

lauren.vinnell@vuw.ac.nz

Dr Taciano Milfont

Victoria University of Wellington

taciano.milfont@vuw.ac.nz

Prof John McClure

Victoria University of Wellington

john.mcclure@vuw.ac.nz 


\section{Appendix 3.B: Study 1 Survey}

This survey will ask a number of questions about natural disasters. For the purpose of this research, a natural disaster is an event or force of nature, such as an earthquake, flood, tsunami, landslide, wildfire, storm, and volcanic eruption, which has severely negative consequences such as causing a lot of damage, injury, and/or disruption to lifelines (e.g., roads, water, electricity, food and medical supplies).

Where you live, how likely is it that a natural disaster will occur in the next five years?

$\begin{array}{ccccc}\text { Very unlikely } & \begin{array}{c}\text { Somewhat } \\ \text { unlikely }\end{array} & \begin{array}{c}\text { Neither likely nor } \\ \text { unlikely }\end{array} & \text { Somewhat likely } & \text { Very likely } \\ \bigcirc & 0 & 0 & \bigcirc & \bigcirc\end{array}$

Suppose that a natural disaster has occurred in your area. How likely do you think it is that the following will occur?

\begin{tabular}{|c|c|c|c|c|c|}
\hline & Very unlikely & $\begin{array}{l}\text { Somewhat } \\
\text { unlikely }\end{array}$ & $\begin{array}{l}\text { Neither likely } \\
\text { nor unlikely }\end{array}$ & $\begin{array}{l}\text { Somewhat } \\
\text { likely }\end{array}$ & Very likely \\
\hline $\begin{array}{l}\text { Major damage to public } \\
\text { facilities (e.g., roads } \\
\text { and parks) in your area }\end{array}$ & 0 & $\bigcirc$ & $\bigcirc$ & 0 & 0 \\
\hline $\begin{array}{l}\text { Supplies (electricity, } \\
\text { communications, water, } \\
\text { etc.) will be interrupted }\end{array}$ & 0 & 0 & 0 & 0 & 0 \\
\hline $\begin{array}{l}\text { Major damage to your } \\
\text { home/possessions }\end{array}$ & 0 & 0 & $\bigcirc$ & 0 & $\Omega$ \\
\hline $\begin{array}{l}\text { You and/or your family } \\
\text { face a life-threatening } \\
\text { situation }\end{array}$ & 0 & 0 & 0 & 0 & $\Omega$ \\
\hline $\begin{array}{l}\text { A prolonged disruption } \\
\text { of your daily life (work } \\
\text { and other daily } \\
\text { activities) }\end{array}$ & $\Omega$ & $\Omega$ & $\Omega$ & $\Omega$ & $\cap$ \\
\hline
\end{tabular}


Have you or someone close to you suffered damage to your home or possessions in a past natural disaster?

(4) A

moderate

(1) None

2

3

amount

$\bigcirc$

O

O

O
5

O
6

(7) A lot

Have you or someone close to you been harmed in a past natural disaster?

(4)
(1) Never
2
3
Moderately
5
6
(7) Severely
$\mathrm{O}$
$\mathrm{O}$
O
O
$\bigcirc$

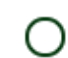

Have you experienced natural events which didn't cause you harm or damage but made you feel scared or vulnerable?

(4) A

moderate

$\begin{array}{llll}\text { (1) Never } & 2 & 3 & \text { number }\end{array}$

$\bigcirc \quad \bigcirc$

$\mathrm{O}$

$\mathrm{O}$

$\bigcirc$
(7) A lot

$\mathrm{O}$ 
Please indicate which of these preparations you or someone in your household have made for the specific purpose of a natural disaster. If you've done one of these in the past but can no longer benefit from it (e.g., stored food years ago which you've since thrown out) please respond 'No'. If you have done part of the action (e.g., purchased a torch but not a battery-powered radio) please respond 'Partly'.

\begin{tabular}{|c|c|c|c|c|c|}
\hline & Yes & No & Partly & Unsure & $\begin{array}{c}\text { Not } \\
\text { applicable }\end{array}$ \\
\hline Store water & $\bigcirc$ & $\bigcirc$ & $\bigcirc$ & $\bigcirc$ & $\bigcirc$ \\
\hline Store non-perishable food & O & O & O & O & O \\
\hline Make an emergency kit & $\bigcirc$ & $\bigcirc$ & $\bigcirc$ & $\bigcirc$ & $\bigcirc$ \\
\hline $\begin{array}{l}\text { Make an emergency plan (e.g., } \\
\text { knowing where to meet family) }\end{array}$ & $\bigcirc$ & O & $\bigcirc$ & O & O \\
\hline $\begin{array}{l}\text { Store supplies (such as plastic bags } \\
\text { and toilet paper) to use as an } \\
\text { emergency toilet }\end{array}$ & $\bigcirc$ & O & O & O & $\bigcirc$ \\
\hline \multirow[t]{2}{*}{$\begin{array}{l}\text { Purchase items to use if power is lost } \\
\text { such as a torch, radio, or gas cooker }\end{array}$} & $\bigcirc$ & $\bigcirc$ & $\bigcirc$ & $\bigcirc$ & $\bigcirc$ \\
\hline & Yes & No & Partly & Unsure & $\begin{array}{c}\text { Not } \\
\text { applicable }\end{array}$ \\
\hline Purchase a water tank & $\bigcirc$ & $\bigcirc$ & $\bigcirc$ & $\bigcirc$ & $\bigcirc$ \\
\hline $\begin{array}{l}\text { Cloud-store important documents } \\
\text { and/or photos on an internet server }\end{array}$ & $\bigcirc$ & $\bigcirc$ & $\bigcirc$ & $\bigcirc$ & $\bigcirc$ \\
\hline Purchase natural disaster insurance & $\bigcirc$ & $\bigcirc$ & $\bigcirc$ & $\bigcirc$ & $\bigcirc$ \\
\hline $\begin{array}{l}\text { Seek out information about the } \\
\text { different natural hazard risks posed to } \\
\text { my home }\end{array}$ & $\bigcirc$ & $\bigcirc$ & $\bigcirc$ & O & $\bigcirc$ \\
\hline $\begin{array}{l}\text { Have the strength of my building } \\
\text { checked (or ask landlord to do the } \\
\text { same) }\end{array}$ & $\bigcirc$ & O & $\bigcirc$ & O & $\bigcirc$ \\
\hline \multirow[t]{2}{*}{ Fasten tall furniture to the wall } & $\bigcirc$ & $\bigcirc$ & $\bigcirc$ & O & $\bigcirc$ \\
\hline & Yes & No & Partly & Unsure & $\begin{array}{c}\text { Not } \\
\text { applicable }\end{array}$ \\
\hline $\begin{array}{l}\text { Secure movable objects in my home } \\
\text { (such as computers and TVs) }\end{array}$ & $\bigcirc$ & $\bigcirc$ & $\bigcirc$ & $\bigcirc$ & $\bigcirc$ \\
\hline $\begin{array}{l}\text { Strengthen my house/its foundations } \\
\text { (or ask landlord to do the same) }\end{array}$ & 0 & O & $\bigcirc$ & $\bigcirc$ & $\bigcirc$ \\
\hline $\begin{array}{l}\text { Ensure that heavy objects are stored } \\
\text { on the floor and at the bottom of } \\
\text { cupboards }\end{array}$ & $\bigcirc$ & $\bigcirc$ & O & $\bigcirc$ & $\bigcirc$ \\
\hline $\begin{array}{l}\text { Identify people in my neighbourhood } \\
\text { who need checking up on in the event } \\
\text { of a natural disaster }\end{array}$ & $\bigcirc$ & $\bigcirc$ & $\bigcirc$ & O & $\bigcirc$ \\
\hline $\begin{array}{l}\text { Store enough emergency supplies to } \\
\text { help others not in my household }\end{array}$ & $\bigcirc$ & $\bigcirc$ & $\bigcirc$ & 0 & $\bigcirc$ \\
\hline $\begin{array}{l}\text { Volunteer my time to help my } \\
\text { community in the event of a disaster } \\
\text { e.g., Community Emergency Hubs }\end{array}$ & $\bigcirc$ & $\bigcirc$ & $\bigcirc$ & $\bigcirc$ & $\bigcirc$ \\
\hline
\end{tabular}


Are there any other actions you have taken specifically for the purpose of being more prepared for a natural disaster? Please write in the text box below:

Please indicate how strongly you agree with the following statements.

I think my family and friends should prepare for a natural disaster.

$\begin{array}{ccccccc}\begin{array}{c}\text { Strongly } \\ \text { disagree }\end{array} & \text { Disagree } & \begin{array}{c}\text { Somewhat } \\ \text { disagree }\end{array} & \begin{array}{c}\text { Neither } \\ \text { agree nor } \\ \text { disagree }\end{array} & \begin{array}{c}\text { Somewhat } \\ \text { agree }\end{array} & \begin{array}{c}\text { Agree } \\ \text { agree }\end{array} \\ 0 & 0 & 0 & 0 & 0 & 0 & \bigcirc\end{array}$

I think people in my neighbourhood should prepare for a natural disaster.

\begin{tabular}{|c|c|c|c|c|c|c|}
\hline $\begin{array}{l}\text { Strongly } \\
\text { disagree }\end{array}$ & Disagree & $\begin{array}{c}\text { Somewhat } \\
\text { disagree }\end{array}$ & $\begin{array}{l}\text { Neither } \\
\text { agree nor } \\
\text { disagree }\end{array}$ & $\begin{array}{c}\text { Somewhat } \\
\text { agree }\end{array}$ & Agree & $\begin{array}{c}\text { Strongly } \\
\text { agree }\end{array}$ \\
\hline 0 & $\bigcirc$ & 0 & $\bigcirc$ & 0 & 0 & 0 \\
\hline
\end{tabular}

I think Wellingtonians should prepare for a natural disaster.

$\begin{array}{ccccccc}\begin{array}{c}\text { Strongly } \\ \text { disagree }\end{array} & \text { Disagree } & \begin{array}{c}\text { Somewhat } \\ \text { disagree }\end{array} & \begin{array}{c}\text { Neither } \\ \text { agree nor } \\ \text { disagree }\end{array} & \begin{array}{c}\text { Somewhat } \\ \text { agree }\end{array} & \text { Agree } & \begin{array}{c}\text { Strongly } \\ \text { agree }\end{array} \\ \bigcirc & 0 & 0 & 0 & 0 & 0 & \bigcirc\end{array}$

I think New Zealanders should prepare for a natural disaster.

$\begin{array}{ccccccc}\begin{array}{c}\text { Strongly } \\ \text { disagree }\end{array} & \text { Disagree } & \begin{array}{c}\text { Somewhat } \\ \text { disagree }\end{array} & \begin{array}{c}\text { Neither } \\ \text { agree nor } \\ \text { disagree }\end{array} & \begin{array}{c}\text { Somewhat } \\ \text { agree }\end{array} & \text { Agree } & \begin{array}{c}\text { Strongly } \\ \text { agree }\end{array} \\ \bigcirc & \bigcirc & 0 & 0 & \bigcirc & \bigcirc & \bigcirc\end{array}$


Please use the slider bars to indicate roughly what percentage of people you think have 'prepared' for a natural disaster (done many of the actions given earlier in the survey) for each group.

$\begin{array}{lllllllllll}0 & 10 & 20 & 30 & 40 & 50 & 60 & 70 & 80 & 90 & 100\end{array}$

Friends and family

People in my neighbourhood

People in Wellington

People in New Zealand 
How strongly do you think your family and friends approve of other people preparing for a natural disaster?

$\begin{array}{ccccccc}\begin{array}{c}\text { Strongly } \\ \text { disapprove }\end{array} & \text { Disapprove } & \begin{array}{c}\text { Somewhat } \\ \text { disapprove }\end{array} & \begin{array}{c}\text { Neither } \\ \text { approve nor } \\ \text { disapprove }\end{array} & \begin{array}{c}\text { Somewhat } \\ \text { approve }\end{array} & \begin{array}{c}\text { Strongly } \\ \text { approve }\end{array} \\ \text { Approve } & 0 & 0 & 0 & 0 & 0 & 0\end{array}$

How strongly do you think people in your neighbourhood approve of other people preparing for a natural disaster?

$\begin{array}{ccccccc}\begin{array}{c}\text { Strongly } \\ \text { disapprove }\end{array} & \text { Disapprove } & \begin{array}{c}\text { Somewhat } \\ \text { disapprove }\end{array} & \begin{array}{c}\text { Neither } \\ \text { approve nor } \\ \text { disapprove }\end{array} & \begin{array}{c}\text { Somewhat } \\ \text { approve }\end{array} & \begin{array}{c}\text { Strongly } \\ \text { approve }\end{array} \\ \text { Ap } & 0 & 0 & 0 & 0 & 0 & 0\end{array}$

How strongly do you think Wellingtonians approve of other people preparing for a natural disaster?

$\begin{array}{ccccccc}\begin{array}{c}\text { Strongly } \\ \text { disapprove }\end{array} & \text { Disapprove } & \begin{array}{c}\text { Somewhat } \\ \text { disapprove }\end{array} & \begin{array}{c}\text { Neither } \\ \text { approve nor } \\ \text { disapprove }\end{array} & \begin{array}{c}\text { Somewhat } \\ \text { approve }\end{array} & \begin{array}{c}\text { Strongly } \\ \text { approve }\end{array} \\ 0 & 0 & 0 & 0 & 0 & 0 & 0\end{array}$

How strongly do you think New Zealanders approve of other people preparing for a natural disaster?

$\begin{array}{ccccccc}\begin{array}{c}\text { Strongly } \\ \text { disapprove }\end{array} & \text { Disapprove } & \begin{array}{c}\text { Somewhat } \\ \text { disapprove }\end{array} & \begin{array}{c}\text { Neither } \\ \text { approve nor } \\ \text { disapprove }\end{array} & \begin{array}{c}\text { Somewhat } \\ \text { approve }\end{array} & \begin{array}{c}\text { Strongly } \\ \text { approve }\end{array} \\ 0 & 0 & 0 & 0 & 0 & 0 & 0\end{array}$


Preparing for natural disasters is:

\begin{tabular}{|c|c|c|}
\hline Useless & 000000 & Useful \\
\hline Unimportant & 0 & Important \\
\hline Unecessary & & Necessary \\
\hline Easy & & Difficult \\
\hline Pointless & & Valuable \\
\hline Smart & & Stupid \\
\hline Simple & & Complicated \\
\hline Unhelpful & $00 \bigcirc \bigcirc 0$ & Helpful \\
\hline
\end{tabular}

How much control do you have over whether you prepare for a natural disaster?

(1) Very little control 2

3

0

$\mathrm{O}$

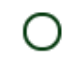

(4) A moderate amount

5

6

(7) Complete control

$\mathrm{O}$

For me to prepare for a natural disaster is...
(1) Easy
2
3
(4) Neither easy
nor difficult
5
6
(7) Difficult
○
O
O
O
O
$\bigcirc$

If I wanted to I could prepare for a natural disaster

(1) Definitely could

2

3
0
O
O

(4) Neither could nor couldn't

5

6

(7) Definitely couldn't

○ 
In this question we would like to ask two related questions. First, for the actions you HAVE done, please indicate how difficult they were, and for the actions your have NOT done, how difficult you think they would be. Please then indicate how effective you think each action is at helping you survive a natural disaster and/or reducing damage in a natural disaster.

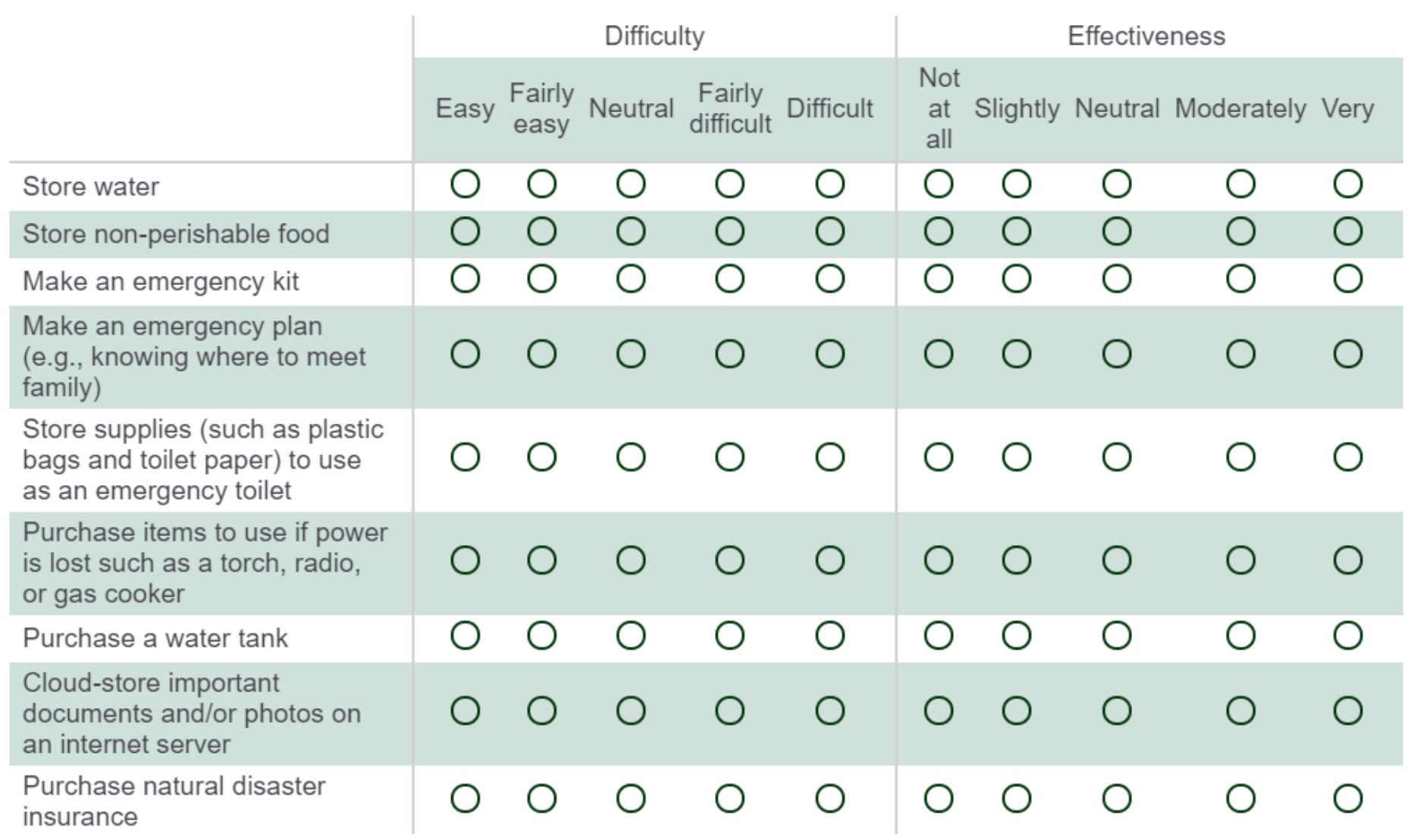


Seek out information about the different natural hazard risks posed to my home

Have the strength of my building checked (or ask landlord to do the same)

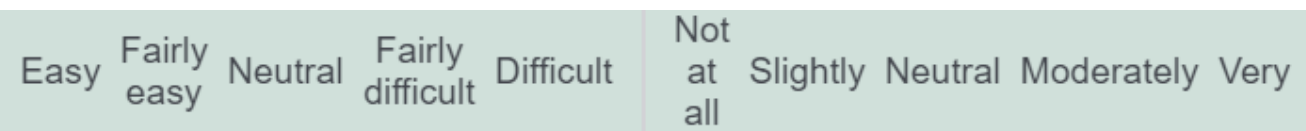
000

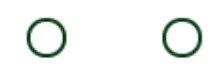
$0 \quad 0 \quad 0$
$\mathrm{O}$

$\begin{array}{llllllllll}0 & 0 & 0 & 0 & 0 & 0 & 0 & 0 & 0 & 0\end{array}$

\begin{tabular}{l|lllllllllll} 
Fasten tall furniture to the wall & $\mathrm{O}$ & $\mathrm{O}$ & $\mathrm{O}$ & $\mathrm{O}$ & $\mathrm{O}$ & $\mathrm{O}$ & $\mathrm{O}$ & $\mathrm{O}$ & $\mathrm{O}$ & $\mathrm{O}$
\end{tabular}

Secure movable objects in my home (such as computers and TVs)

Strengthen my house/its foundations (or ask landlord to do the same)

Ensure that heavy objects are stored on the floor and at the bottom of cupboards

Identify people in my neighbourhood who need checking up on in the event of $\begin{array}{llllllll}0 & 0 & 0 & 0 & 0 & 0 & 0 & 0\end{array}$ 000 a natural disaster

\begin{tabular}{|c|c|c|c|c|c|c|c|c|}
\hline $\begin{array}{l}\text { Store enough emergency } \\
\text { supplies to help others not in } \\
\text { my household }\end{array}$ & $\bigcirc \bigcirc$ & 0 & 0 & 0 & 0 & 0 & $\bigcirc$ & 0 \\
\hline $\begin{array}{l}\text { Volunteer my time to help my } \\
\text { community in the event of a } \\
\text { disaster e.g., Community } \\
\text { Emergency Hubs }\end{array}$ & $\bigcirc \bigcirc$ & 0 & $\bigcirc$ & 0 & $\bigcirc$ & $\bigcirc$ & $\bigcirc$ & $\bigcirc$ \\
\hline
\end{tabular}


How old are you?

What is your gender?
Male
$\bigcirc$ Female
Other:

What is your ethnicity? You may select multiple ethnicities if you identify with more than one
$\square$ NZ European/Pākehā
$\square$ North American
$\square$ Asian (please specify)
$\square$ South/Latin American
$\square$ Pasifika
$\square$ African (please specify)
$\square$ Māori
$\square$ Other (please specify)
European (please specify)

What is the highest level of education you have completed?
Secondary school incomplete
Postgraduate degree
NCEA, School Cert, or other secondary school qualification
Masters degree
Polytechnic or Trade Certificate
Doctorate
Undergraduate degree 
What is your annual income before tax?
$\bigcirc$ Below $\$ 20,000$
$\$ 70,000$ to $\$ 79,999$
$\$ 20,000$ to $\$ 29,999$
$\$ 80,000$ to $\$ 89,999$
$\$ 30,000$ to $\$ 39,999$
$\$ 90,000$ to $\$ 99,999$
$\$ 40,000$ to $\$ 49,999$
$\$ 100,000+$
$\$ 50,000$ to $\$ 59,999$
Prefer not to say
$\$ 60,000$ to $\$ 69,999$

Does your (collective family) income cover your household costs?
No, costs are considerably higher
$\mathrm{No}$, costs are slightly higher
Yes, income and costs are similar
$\mathrm{Y}$ Yes, with a small surplus
$\bigcirc$ Yes, comfortably

Which suburb of Wellington do you live in?

What is your current living situation?
$\bigcirc$ Parents' home
Home you own
Renting
Social housing
$\mathrm{OHostel} / \mathrm{Hall}$ s of residence
Other 

House (free-standing)
$\bigcirc$ Unit/flat (joined to another unit/flat)
Apartment in multi-level building
Moveable dwelling, for example, caravan, boat, tent etc
Other, please specify

Project title: Understanding why people in Wellington prepare for natural disasters

Thank you for participating in this research.

This study examines natural disaster preparation in Wellington. It explores the different barriers that might contribute to people not preparing, as well as other related factors such as experience and perceived risk. One goal of this survey is to understand the reasons why people do or do not prepare for natural disasters in Wellington. This will help us to understand what behaviours need targeting and how to go about targeting them.

Some participants in this survey answered questions about place attachment (emotional bond to a place), while others answered questions about the effectiveness and difficulty of preparing for a disaster. Previous research has shown that these factors, as well as social norms (what behaviours are common and desirable in a social group), contribute to disaster preparation and mitigation. The information we collect in this survey will help us to understand if we can use these factors to purposefully increase disaster preparation.

We hope that the information collected in this survey will help us to construct interventions to encourage Wellingtonians to prepare more for natural disasters. . Should these interventions be successful, they can then be applied to a wider population through advising and working with relevant organizations such as the EQC, Civil Defence, and local councils. 
Appendix 4.A: Study 2 Disaster condition information sheet

\section{Application ID Number: 000026244}

\author{
Lauren Vinnell \\ PhD Student \\ Email: lauren.vinnell@vuw. \\ ac.nz
}

\author{
A.Prof. Taciano Milfont \\ Primary Supervisor \\ Email: taciano.milfont@vuw. \\ ac.nz
}

Phone: (04) 4636398

\author{
Prof. John McClure \\ Secondary Supervisor \\ Email: john.mcclure@vuw. \\ ac.nz \\ Phone: (04) 4635233 ext \\ 6047
}

\section{What is the purpose of this research?}

- This research will help us to understand why people in Wellington do and do not prepare for natural disasters, including their beliefs about whether it's a common and approved behaviour.

\section{Who is conducting the research?}

-We are a team of researchers in the School of Psychology at Victoria University of Wellington. PhD student Lauren Vinnell is leading this project. Associate Professor Taciano Milfont and Professor John McClure are supervising. This research has been approved by the School of Psychology Human Ethics Committee under delegated authority of Victoria University of Wellington's Human Ethics Committee (application number: 000026244, convenor: Prof. Paul Jose).

\section{What is involved if you agree to participate?}

- If you agree to participate in this study, you will complete a short survey where you will respond to a range of different questions such as "Where you live, how likely is it that a natural disaster will occur in the following five years?" as well as letting us know what preparations you've made, and which ones you intend to do.

- Some questions will ask you about your previous or potential future experiences of natural disasters which might cause emotional discomfort such as "Have you or someone close to you been harmed in a past natural disaster?". You do not have to answer these questions if you are concerned about how they will make you feel.

-We will also ask you for some demographic information e.g., age, income, ethnicity. This is so that we can have a deeper understanding of the additional factors that may impact on natural disaster preparation.

-We anticipate that the survey will take you no more than 20 minutes to complete.

- During the research you are free to withdraw at any point before your survey has been completed.

- As a token of appreciation, you will be given the opportunity to go into the draw for one of three $\$ 100$ supermarket gift cards.

\section{Privacy and Confidentiality}

- This survey is completely confidential. We want to make your responses unidentifiable so please do not enter any identifying information in the text boxes.

- Digital copies of your responses will be kept for five years after the publication of this research at which point they will then be destroyed. On the first page, you can request your survey responses be destroyed as soon as the data has been extracted. 
- In accordance with the requirements of some scientific journals and organizations, information from your survey that does not identify you may be shared with other researchers.

- An electronic version of the data used for analyses will remain indefinitely in the custody of the researchers at Victoria University of Wellington. This data will not contain any identifying information.

- At the end of the survey, you will have the option to provide your email address to enter the prize draw for one of three $\$ 100$ supermarket gift cards. This information will be kept separate from the survey data so that your responses cannot be linked to you. Email addresses will be held securely on a password-protected database and destroyed immediately after prizes have been allocated (approximately 2 weeks after the survey closes). Any other identifying information will be destroyed once the dataset has been collated.

- On the first page, you will be asked to consent to participate in this study. You will also be asked if you'd like to join a mailing list to receive a follow-up survey in the unfortunate event of a natural disaster occurring in the near future, as this would give us a valuable opportunity to study the impacts of such events. If you agree to this, your contact details will be entered into a spreadsheet which will be stored indefinitely on a password-protected University computer. You can contact any of the research team at any time to have your details removed from this list if you change your mind. This information will not be included in the data and will not be shared with anyone outside of the research team.

\section{What happens to the information that you provide?}

- The overall findings will form part of a PhD thesis, and/or be published in scientific journals, presented at academic conferences, and/or grant applications.

If you would like to know the results of this study, they will be available approximately March, 2019 through A.Prof Taciano Milfont's research lab website (www.vuwenvironmentallab.wordpress.com).

Thank you for considering participation in this research. 


\section{Appendix 4.B: Study 2 Disaster condition survey}

\section{Understanding why people in Wellington prepare for natural disasters}

Thank you for considering taking part in this short survey.

The purpose of this research is to understand why people in Wellington do or do not prepare for natural disasters. You don't need to have done any preparation yourself, just read the following questions carefully and give the answer which best reflects your opinion. Please try to answer all of the questions. In order to complete this survey you must be currently living in Wellington and be 18 years of age or older.

The survey is completely confidential, so your responses cannot be identified. We anticipate that this research will take you no more than 15 to 20 minutes to complete. At the end, you'll have the chance to go into the prize draw for one of three $\$ 100$ supermarket gift cards.

If you would like to know more about the study before proceeding, please click here.

I have read the information above and hereby give my consent to participate in this research. By participating I confirm that I am over the age of 18. I understand that my responses will be kept confidential and that I can withdraw from the study at any point. All identifying information will either be destroyed or stored securely, as explained in the information sheet and in accordance with the option you choose below. Your contact details (email address) will ONLY potentially be re-used if you select the third of the following options.

Please select one of the following options:

I want my survey responses and all identifying information destroyed as soon as the data has been extracted

My survey responses can be kept for five years but I want all identifying information destroyed immediately after prizes have been allocated

My contact details can be kept on a secure mailing list so I can be contacted in future to participate in another similar study (you will be prompted to provide your email address at the end of this survey)

Please select one of the following options:

$\bigcirc$ Yes, I agree to participate

$\bigcirc$ No, I do not want to participate 
Do you currently live in the Wellington region (including Wellington City, Upper and Lower Hutt, and Porirua)?

$\begin{array}{ll}\text { Yes } & \text { No } \\ \bigcirc & \bigcirc\end{array}$

This survey will ask a number of questions about natural disasters. For the purpose of this research, a natural disaster is an event or force of nature, such as an earthquake, flood, tsunami, landslide, wildfire, storm, and volcanic eruption, which has severely negative consequences such as causing a lot of damage, injury, and/or disruption to lifelines (e.g., roads, water, electricity, food and medical supplies).

Do you understand this definition?

Some of the questions you will see in this survey may seem to be similar, but they do ask for slightly different thinking and are all important. Please read each question and the available responses carefully.

Please answer the following questions by selecting the response which best reflects your agreement with the statement.

\begin{tabular}{|c|c|c|c|c|c|c|}
\hline & $\begin{array}{l}\text { Strongly } \\
\text { disagree }\end{array}$ & 2 & 3 & 4 & 5 & $\begin{array}{c}\text { Strongly } \\
\text { agree }\end{array}$ \\
\hline I plan to prepare for a natural disaster & $\bigcirc$ & & & & & O \\
\hline I want to prepare for a natural disaster & 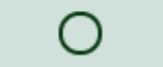 & & & & & \\
\hline I intend to prepare for a natural disaster & $\bigcirc$ & 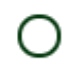 & & & & C \\
\hline I expect to prepare for a natural disaster & O & & & & & $\bigcirc$ \\
\hline
\end{tabular}


Please answer the following questions by selecting the response which best reflects your opinion about the statement.

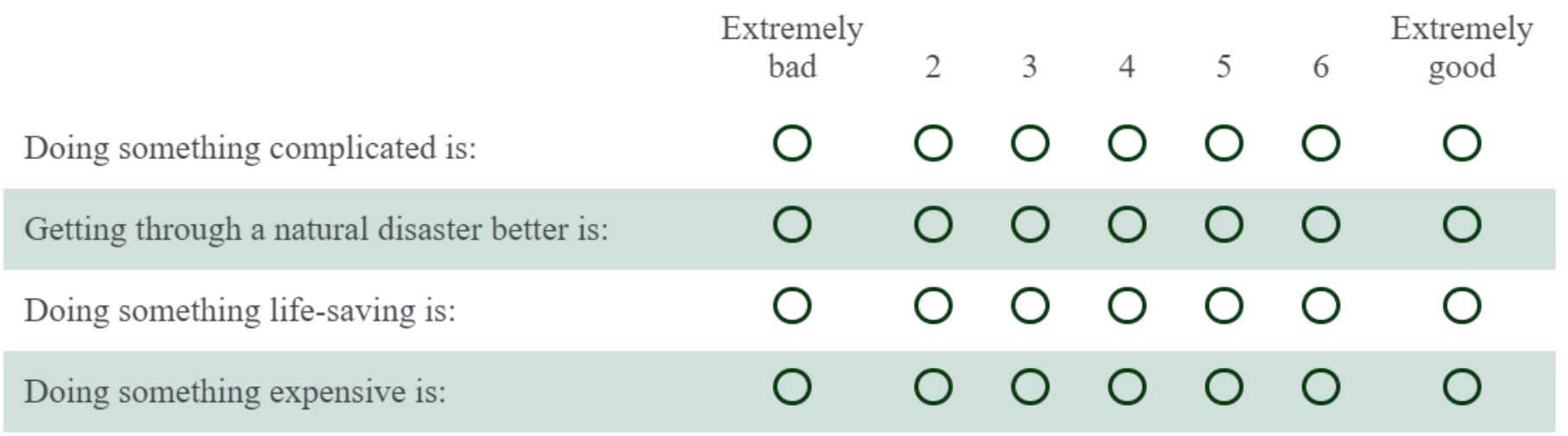

I think that preparing for natural disasters is:

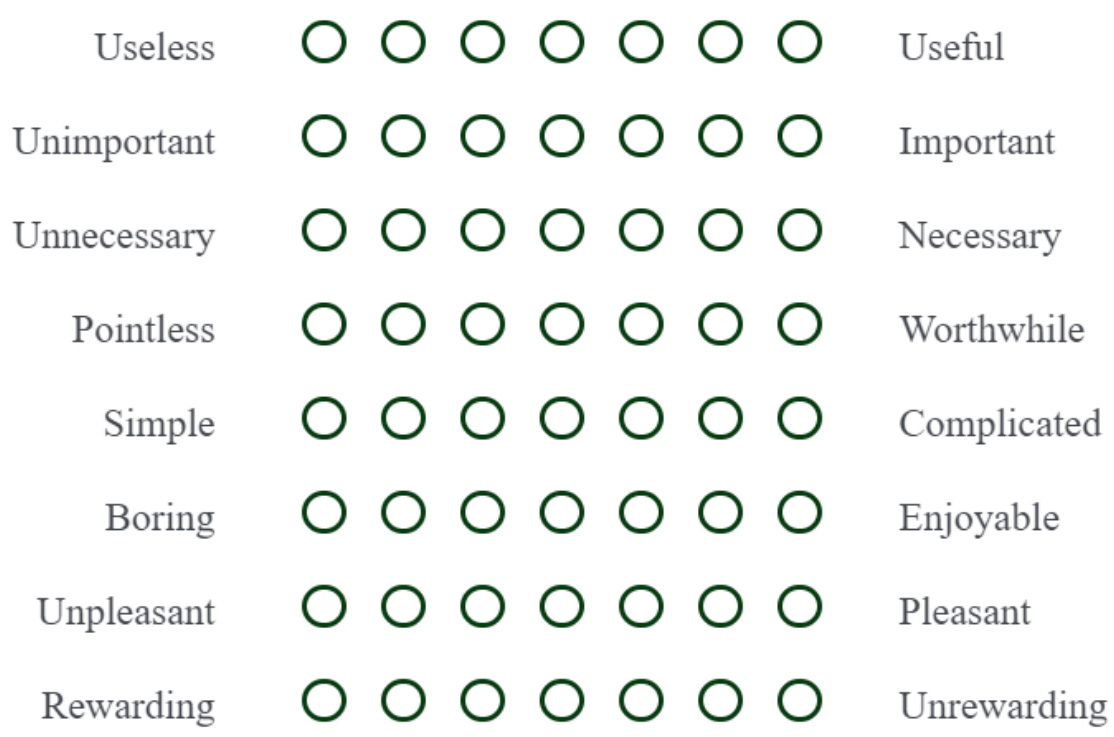


Appendices

Please answer the following questions by selecting the response which best reflects your agreement with the statement.

$$
\begin{array}{llllllll}
\begin{array}{c}
\text { Strongly } \\
\text { disagree }
\end{array} & 2 & 3 & 4 & 5 & 6 & \begin{array}{c}
\text { Strongly } \\
\text { agree }
\end{array}
\end{array}
$$

Preparing for a natural disaster will result in me $\quad \begin{array}{lllllll}0 & 0 & 0 & 0 & 0 & 0\end{array}$ and my home getting through a disaster better

It is complicated to prepare for a natural disaster $\quad \begin{array}{llllll}0 & 0 & 0 & 0\end{array}$

It is expensive to prepare for a natural disaster

$\begin{array}{lllllll}0 & 0 & 0 & 0 & 0 & 0 & 0\end{array}$

I will feel like I'm doing something life-saving if I prepare for a natural disaster

$\begin{array}{lllllll}0 & 0 & 0 & 0 & 0 & 0 & 0\end{array}$

Most people who are important to me think that prepare for a natural disaster
I should
2
3
4
5
6
I should NOT
○
O
O
O
O
O
O

Please answer the following questions by selecting the response which best reflects your agreement with the statement.

$\begin{array}{llllllll}\begin{array}{l}\text { Strongly } \\ \text { disagree }\end{array} & 2 & 3 & 4 & 5 & 6 & \begin{array}{c}\text { Strongly } \\ \text { agree }\end{array}\end{array}$

It is expected of me that I prepare for a natural disaster
$\mathrm{O}$
O

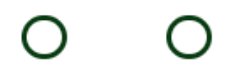
00
$\mathrm{O}$

I feel under social pressure to prepare for a natural disaster
O
O
$\bigcirc \bigcirc$
O
O
O

People who are important to me want me to prepare for a natural disaster

$\begin{array}{lllllll}0 & 0 & 0 & 0 & 0 & 0\end{array}$ 
Among the people who are important to you, how much agreement would there be that preparing for a natural disaster is a good thing to do?

No

agreement

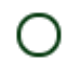

2

0
3

0
4

0
5

0
6

0
A great deal of agreement

How many of the people who are important to you do you think have prepared for a natural disaster?

$\begin{array}{ccccccc}\text { None } & 2 & 3 & 4 & 5 & 6 & \text { All } \\ 0 & 0 & 0 & \bigcirc & 0 & \bigcirc & \bigcirc\end{array}$

Please answer the following questions by selecting the response which best reflects your agreement with the statement. By 'prepared', we mean having done a number of different actions for the event of a natural disaster, not just one or two.

$\begin{array}{lllllll}\begin{array}{c}\text { Strongly } \\ \text { disagree }\end{array} & 2 & 3 & 4 & 5 & 6 & \begin{array}{c}\text { Strongly } \\ \text { agree }\end{array}\end{array}$

Most people who are important to me approve

of my preparing for a natural disaster

$\bigcirc \bigcirc \bigcirc \bigcirc \bigcirc \bigcirc$

Most people like me approve of my preparing for a natural disaster

000000

○

The people in my life whose opinions I value approve of my preparing for a natural disaster

00000000

Most people who are important to me have prepared for a natural disaster

000000

Most people like me have prepared for a natural disaster

0000000

The people in my life whose opinions I value have prepared for a natural disaster

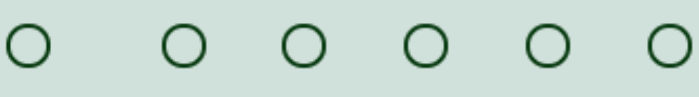


Please answer the following questions by selecting the response which best reflects your agreement with the statement.

$\begin{array}{llllllll}\begin{array}{l}\text { Strongly } \\ \text { disagree }\end{array} & 2 & 3 & 4 & 5 & 6 & \begin{array}{c}\text { Strongly } \\ \text { agree }\end{array}\end{array}$

My family and friends approve of my preparing for a natural disaster

$\bigcirc \quad \bigcirc \quad \bigcirc \quad \bigcirc \quad 0 \quad 0$

When it comes to preparing for a natural disaster, I want to do what my
○
$\bigcirc \bigcirc$
$\bigcirc$
O
O

O

neighbours think I should do

When it comes to preparing for a natural disaster, I want to do what other

Wellingtonians think I should do

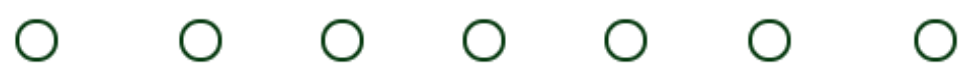

When it comes to preparing for a natural disaster, I want to do what other

New Zealanders think I should do

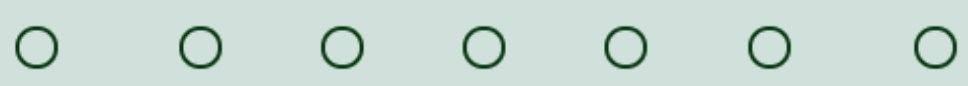

New Zealanders approve of my

preparing for a natural disaster

$\begin{array}{lllllll} & 0 & 0 & 0 & 0 & 0 & 0\end{array}$

Wellingtonians approve of my preparing for a natural disaster
○
$\bigcirc$
$\bigcirc$
$\bigcirc$
O
$\bigcirc$
O

My neighbours approve of my preparing for a natural disaster

O 0000000

When it comes to preparing for a natural disaster, I want to do what my

000000

family and friends think I should do 
Please answer the following questions by selecting the response which best reflects your opinion about the statement.

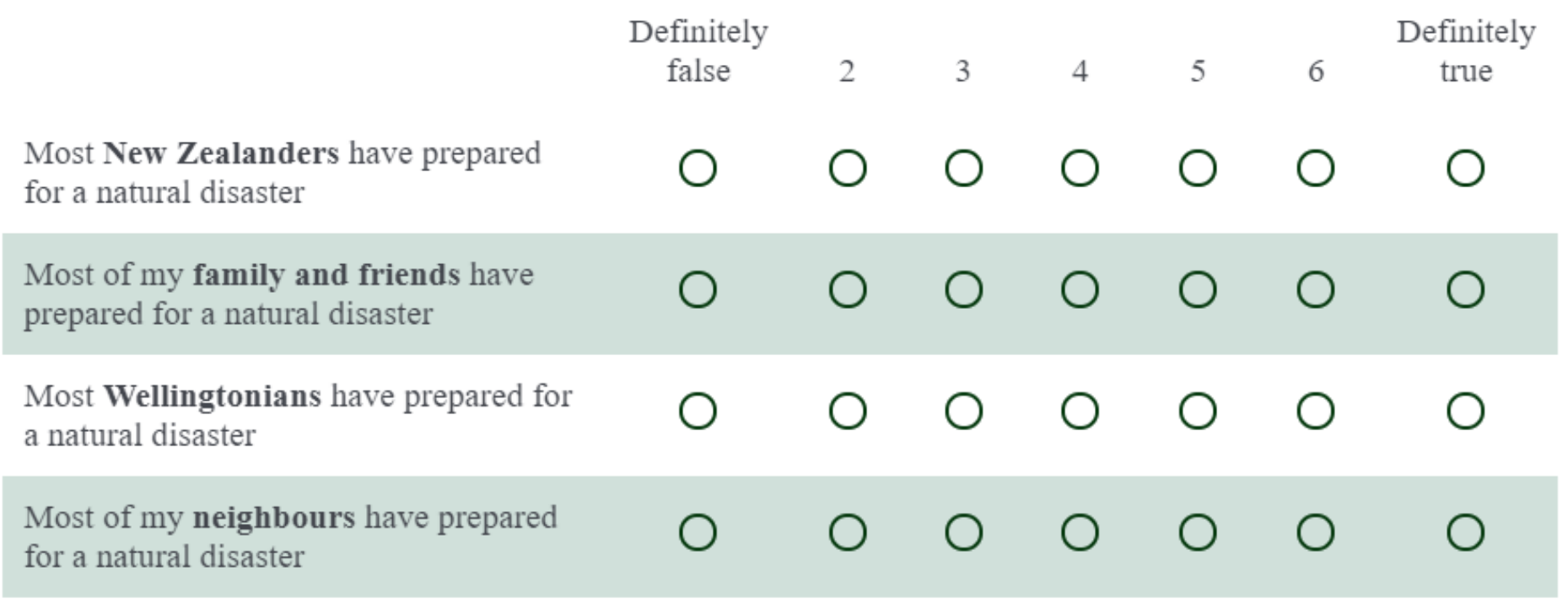

Please answer the following questions by selecting the response which best reflects your opinion about the statement.

Very

much

23

Not at

all

When it comes to preparing for a natural disaster, how much do you want to be like your neighbours?

When it comes to preparing for a natural disaster, how much do you want to be like other Wellingtonians?
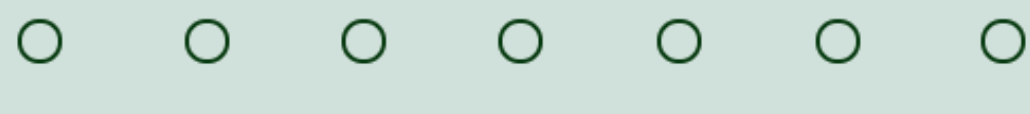

When it comes to preparing for a natural disaster, how much do you want to be like other New Zealanders?

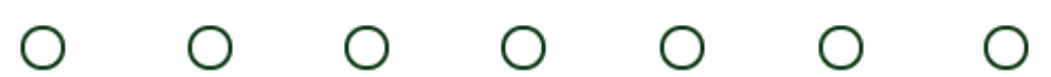

When it comes to preparing for a natural disaster, how much do you want to be like your family and friends?
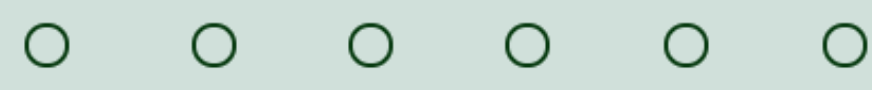
How much control do you believe you have over preparing for a natural disaster?

$\begin{array}{ccccccc}\begin{array}{c}\text { No } \\ \text { control }\end{array} & 2 & 3 & 4 & 5 & 6 & \begin{array}{r}\text { Complete } \\ \text { control }\end{array} \\ \bigcirc & \bigcirc & \bigcirc & \bigcirc & \bigcirc & \bigcirc & \bigcirc\end{array}$

Please answer the following questions by selecting the response which best reflects your agreement with the statement.

If I wanted to I could prepare for a natural
disaster

For me to prepare for a natural disaster is...

Definitely

impossible

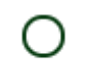

2

0
3

O
4

0
5

0
Definitely possible

0

For me to prepare for a natural disaster is...

$\begin{array}{ccccccc}\begin{array}{c}\text { Extremely } \\ \text { easy }\end{array} & 2 & 3 & 4 & 5 & 6 & \begin{array}{c}\text { Extremely } \\ \text { difficult }\end{array} \\ \bigcirc & \bigcirc & \bigcirc & \bigcirc & \bigcirc & \bigcirc & \bigcirc\end{array}$


Please answer the following questions by selecting the response which best reflects your opinion of the likelihood of the statement.

$\begin{array}{ccccccc}\begin{array}{c}\text { Extremely } \\ \text { unlikely }\end{array} & 2 & 3 & 4 & 5 & 6 & \begin{array}{c}\text { Extremely } \\ \text { likely }\end{array}\end{array}$

In the next month, I will have the money to prepare for a natural disaster

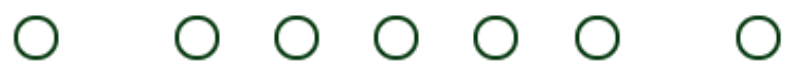

In the next month, I will make the effort to prepare for a natural disaster

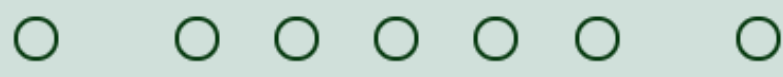

In the next month, I will have the time to prepare for a natural disaster

0000000

In the next month, I will think about preparing for a natural disaster

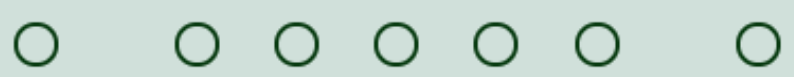

Please answer the following questions by selecting the response which best reflects your agreement with the statement.

$\begin{array}{lllllll}\begin{array}{l}\text { Strongly } \\ \text { disagree }\end{array} & 2 & 3 & 4 & 5 & 6 & \begin{array}{c}\text { Strongly } \\ \text { agree }\end{array}\end{array}$

Having enough money would make me more likely to prepare for a natural disaster

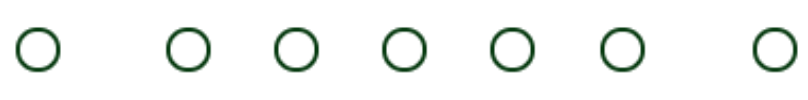

Having the time would make me more likely to prepare for a natural disaster
○
O
O
000

O

Thinking about preparing would make me more likely to prepare for a natural disaster

000000

Making the effort to prepare for a natural disaster would make me more likely to prepare

000000 
Appendices

How likely is it that a natural disaster will occur where you live in the next five years?

$\begin{array}{ccccc}\text { Very unlikely } & \text { Somewhat unlikely } & \begin{array}{c}\text { Neither likely nor } \\ \text { unlikely }\end{array} & \text { Somewhat likely } & \text { Very likely } \\ \bigcirc & \bigcirc & \bigcirc & \bigcirc & \bigcirc\end{array}$

Suppose that a natural disaster has occurred in your area. How likely do you think it is that the following will occur?

\begin{tabular}{|c|c|c|c|}
\hline Very & Somewhat & $\begin{array}{l}\text { Neither } \\
\text { likely nor }\end{array}$ & Somewhat \\
\hline
\end{tabular}

Major damage to public facilities (e.g., roads and parks) in your area
$\bigcirc$
O
O
O
O

A prolonged disruption of your daily life (work and other daily activities)

$0 \quad 0 \quad 0$

O

Supplies (electricity, communications, water, etc.) will be interrupted

$\bigcirc \quad \bigcirc \quad \bigcirc$

$\bigcirc$

You and/or your family face a lifethreatening situation

O $\mathrm{O}$

O

O

Major damage to your

home/possessions 
Have you experienced natural events which didn't cause you harm or damage but made you feel scared or vulnerable?

$\begin{array}{ccccccc}\text { None } & 2 & 3 & 4 & 5 & 6 & \text { Many } \\ \bigcirc & \bigcirc & \bigcirc & \bigcirc & \bigcirc & \bigcirc & \bigcirc\end{array}$

Have you or someone close to you been harmed in a past natural disaster?

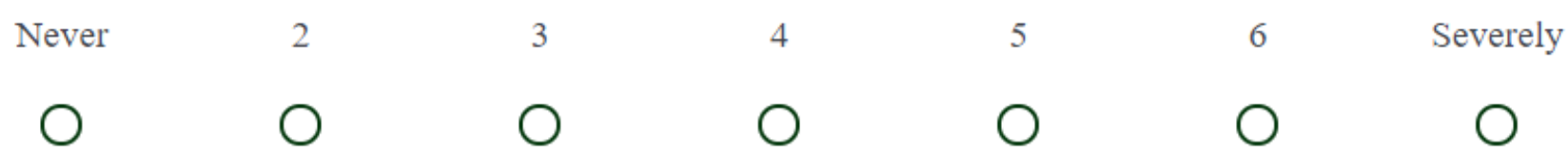

Have you or someone close to you suffered damage to your home or possessions in a past natural disaster?

$\begin{array}{ccccccc}\begin{array}{c}\text { No } \\ \text { damage }\end{array} & 2 & 3 & 4 & 5 & 6 & \begin{array}{l}\text { A lot of } \\ \text { damage }\end{array} \\ \bigcirc & \bigcirc & \bigcirc & \bigcirc & 0 & 0 & \bigcirc\end{array}$

During the past month, how much preparation have you done for a natural disaster?

$\begin{array}{rrrrrrr}\text { None } & 2 & 3 & 4 & 5 & 6 & \text { A lot } \\ & 0 & 0 & \bigcirc & 0 & \bigcirc & \bigcirc\end{array}$


Please indicate which of these preparations you or someone in your household have made for the specific purpose of a natural disaster. If you've done one of these in the past but can no longer benefit from it (e.g., stored food years ago which you've since thrown out) please respond 'No'. If you have done part of the action (e.g., purchased a torch but not a battery-powered radio) please respond 'Partly'.

\begin{tabular}{|c|c|c|c|c|c|}
\hline & Yes & No & Partly & Unsure & $\begin{array}{c}\text { Not } \\
\text { applicable }\end{array}$ \\
\hline Store water & $\cap$ & 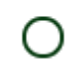 & $\bigcirc$ & $\bigcirc$ & $\bigcirc$ \\
\hline $\begin{array}{l}\text { Store enough emergency supplies to help others not } \\
\text { in my household }\end{array}$ & & & & & \\
\hline Purchase natural disaster insurance & & & $\bigcirc$ & $\bigcirc$ & ○ \\
\hline $\begin{array}{l}\text { Make an emergency plan (e.g., knowing where to } \\
\text { meet family) }\end{array}$ & & & & $\Omega$ & ) \\
\hline $\begin{array}{l}\text { Identify people in my neighbourhood who need } \\
\text { checking up on in the event of a natural disaster }\end{array}$ & & & & 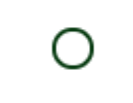 & ) \\
\hline \multirow[t]{2}{*}{ Store non-perishable food } & & & & 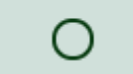 & ) \\
\hline & Yes & No & Partly & Unsure & $\begin{array}{l}\text { Not } \\
\text { applicable }\end{array}$ \\
\hline Purchase a water tank & & & D & $\bigcirc$ & D \\
\hline Fasten tall furniture to the wall & & & $\bigcirc$ & $\bigcirc$ & $\bigcirc$ \\
\hline $\begin{array}{l}\text { Strengthen my house/its foundations (or ask } \\
\text { landlord to do the same) }\end{array}$ & & & $\bigcirc$ & $\bigcirc$ & $\bigcirc$ \\
\hline $\begin{array}{l}\text { Purchase items to use if power is lost such as a } \\
\text { torch, radio, or gas cooker }\end{array}$ & & & & $\mathrm{O}$ & $\mathrm{O}$ \\
\hline $\begin{array}{l}\text { Secure movable objects in my home (such as } \\
\text { computers and TVs) }\end{array}$ & & & & & \\
\hline \multirow[t]{2}{*}{$\begin{array}{l}\text { Seek out information about the different natural } \\
\text { hazard risks posed to my home }\end{array}$} & & & & & \\
\hline & Yes & No & Partly & Unsure & $\begin{array}{l}\text { Not } \\
\text { applicable }\end{array}$ \\
\hline $\begin{array}{l}\text { Identify my local Community Emergency Hub so I } \\
\text { can volunteer in the event of a natural disaster }\end{array}$ & $\curvearrowleft$ & & $\curvearrowleft$ & $\bigcirc$ & $\bigcirc$ \\
\hline $\begin{array}{l}\text { Have the strength of my building checked (or ask } \\
\text { landlord to do the same) }\end{array}$ & 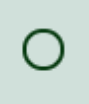 & $\cap$ & $\frown$ & 0 & 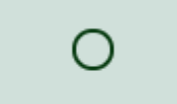 \\
\hline Make an emergency kit & & $\mathrm{O}$ & 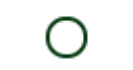 & O & $\bigcirc$ \\
\hline
\end{tabular}


Store supplies (such as plastic bags and toilet paper) to use as an emergency toilet

Ensure that heavy objects are stored on the floor and at the bottom of cupboards

Cloud-store important documents and/or photos on an internet server
$\mathrm{O} \quad \mathrm{O}$

$\mathrm{O} \quad \mathrm{O}$

$\bigcirc \quad \bigcirc$
○

○

O

0

Are there any other actions you have taken specifically for the purpose of being more prepared for a natural disaster? Please write in the text box below:

How old are you?

What is your gender?
Male
$\bigcirc$ Female
Other:

With which ethnicity do you most strongly identify?
NZ European/Pākehā
North American
Asian (please specify)
South/Latin American
Pasifika
African (please specify)
Māori
$\bigcirc$ Other (please specify)
$\bigcirc$ European (please specify) 
What is the highest level of education you have completed?

Secondary school incomplete

Postgraduate degree

NCEA, School Cert, or other secondary school qualification

Masters degree

Polytechnic or Trade Certificate

Doctorate

$\bigcirc$ Undergraduate degree

What is your annual income before tax?
$\bigcirc$ Below $\$ 20,000$
$\$ 70,000$ to $\$ 79,999$
$\$ 20,000$ to $\$ 29,999$
O $\$ 80,000$ to $\$ 89,999$
$\bigcirc 30,000$ to $\$ 39,999$
$\bigcirc \$ 90,000$ to $\$ 99,999$
$\bigcirc 40,000$ to $\$ 49,999$
$\bigcirc 100,000+$
$\bigcirc 50,000$ to $\$ 59,999$
$\bigcirc$ Prefer not to say
$\$ 60,000$ to $\$ 69,999$

Does your (collective family) income cover your household costs?
No, costs are considerably higher
$\bigcirc$ No, costs are slightly higher
$\bigcirc$ Yes, income and costs are similar
$\bigcirc$ Yes, with a small surplus
Yes, comfortably

Which suburb of Wellington do you live in? 
What is your current living situation?
$\bigcirc$ Parents' home
Home you own
Renting
Social housing
Hostel/Halls of residence
Other

Which of these options best describe your housing situation?
House (free-standing)
Unit/flat (joined to another unit/flat)
$\bigcirc$ Apartment in multi-level building
Moveable dwelling, for example, caravan, boat, tent etc
$\bigcirc$ Other, please specify 


\section{Application ID Number: 000026244}

Thank you for participating in this survey.

This study examines natural hazard preparation in Wellington. It explores the different barriers that might contribute to people not preparing, as well as other related factors such as experience of natural hazards and perceived hazard risk. One goal of this survey is to understand the reasons why people do and do not prepare for natural hazards in Wellington. This will help us to understand what thoughts need to be targeted so that we can increase preparation behavior.

We are also interested in whether people think about natural hazards and natural disasters in different ways. There are two versions of this survey, each using one of the different terms. Understanding the effect of these words, if there is an effect, can help us to make communications more effective.

This survey assessed your attitudes towards natural hazard preparation, your perception of social norms (is natural hazard preparation common and desirable in your social groups), as well as whether you think you have control over your own natural hazard preparation. The information we collect in this survey will help us to understand if we should target these factors to purposefully increase natural hazard preparation.

We hope that the information collected in this survey will help us to construct interventions to encourage Wellingtonians to prepare more for natural hazards. Should these interventions be successful, they can then be applied to a wider population through advising and working with relevant organizations such as the EQC, Civil Defence, and local councils.

This research was approved by the School of Psychology Human Ethics Committee under delegated authority of Victoria University of Wellington's Human Ethics Committee (application number: 000026244, convenor: Prof. Paul Jose).

Thank you again for participating in this research.

Lauren Vinnell

PhD Student

School of Psychology

Victoria University of Wellington 
To whom it may concern,

You have been randomly chosen to participate in a survey being conducted by a team at Victoria University of Wellington's School of Psychology. We are interested in understanding why people in Wellington do and do not prepare for natural hazards. At the bottom of this page, you will find a link which will take you to the survey.

We think this survey will take about 15 minutes to answer, and if you complete it you'll have the option to go into the draw for one of three $\$ 100$ supermarket gift cards. You'll also help us to understand how we can best encourage people in Wellington to prepare for natural hazards.

Thank you for considering participating in this research. If you have any questions, please feel free to contact one of the research team below.

If you would like to participate, please type the following into a web browser on any Internet-enabled device: https://tinyurl.com/y27munyg

Many thanks,

\section{Lauren Vinnell PhD Student}

Email:

lauren.vinnell@vuw.ac.nz

\section{A. Prof Taciano Milfont Primary Supervisor} Email:

taciano.milfont@vuw.ac.nz
Prof. John McClure Secondary Supervisor Email:

john.mcclure@vuw.ac.nz 
Appendix 5.B: Study 3 Time 1 information sheet

\section{Application ID Number: 000026244}

\author{
Lauren Vinnell \\ PhD Student \\ Email: \\ lauren.vinnell@vuw.ac.nz
}

\author{
A.Prof. Taciano Milfont \\ Primary Supervisor \\ Email: \\ taciano.milfont@vuw.ac.nz \\ Phone: (04) 4636398
}

\author{
Prof. John McClure \\ Secondary Supervisor \\ Email: \\ john.mcclure@vuw.ac.nz \\ Phone: (04) 4636047
}

\section{What is the purpose of this research?}

- This research will help us to understand why people in Wellington do and do not prepare for natural hazards, including their beliefs about whether it's a common and approved behaviour.

\section{Who is conducting the research?}

- We are a team of researchers in the School of Psychology at Victoria University of Wellington. PhD student Lauren Vinnell is leading this project. Associate Professor Taciano Milfont and Professor John McClure are supervising. This research has been approved by the School of Psychology Human Ethics Committee under delegated authority of Victoria University of Wellington's Human Ethics Committee (application number: 000026244, convenor: Prof. Paul Jose).

\section{What is involved if you agree to participate?}

- If you agree to participate in this study, you will complete a short survey where you will respond to a range of different questions such as "How much control do you believe you have over preparing for a natural hazard?" as well as letting us know what preparations you've made, and whether you intend to do more.

- Some questions will ask you about your previous or potential future experiences of natural hazards which might cause emotional discomfort such as "Have you or someone close to you been harmed in a past natural hazard?". You do not have to answer these questions if you are concerned about how they will make you feel.

-We will also ask you for some demographic information e.g., age, education, ethnicity. This is so that we can have a deeper understanding of the additional factors that may impact on natural hazard preparation.

-We anticipate that the survey will take you about 15 minutes to complete.

- During the research you are free to withdraw at any point before your survey has been completed. 
- As a token of appreciation, you will be given the opportunity to go into the draw for one of three $\$ 100$ supermarket gift cards.

\section{Privacy and Confidentiality}

- This survey is completely confidential.

- Digital copies of your responses will be kept for five years after the publication of this research at which point they will then be destroyed. On the first page, you can request your survey responses be destroyed as soon as the data has been extracted.

- In accordance with the requirements of some scientific journals and organizations, information from your survey that does not identify you may be shared with other researchers.

- An electronic version of the data used for analyses will remain indefinitely in the custody of the researchers at Victoria University of Wellington. This data will not contain any identifying information.

- At the end of the survey, you will have the option to provide your email address to enter the prize draw for one of three $\$ 100$ supermarket gift cards. This information will be kept separate from the survey data so that your responses cannot be linked to you. Email addresses will be held securely on a password-protected database and destroyed immediately after prizes have been allocated (approximately 2 months after the survey closes). Any other identifying information will be destroyed once the dataset has been collated.

- On the first page, you will be asked to consent to participate in this study. You will also be asked if you'd like to join a mailing list to receive a follow-up survey in one month's time. This survey will be much shorter, and completing it will give you a second entry into the prize draw. This follow-up survey is very important to understand why people do and do not prepare for natural hazards. If you agree to this, your contact details will be entered into a spreadsheet which will be stored indefinitely on a password-protected University computer. You can contact any of the research team at any time to have your details removed from this list if you change your mind. This information will not be included in the data and will not be shared with anyone outside of the research team.

\section{What happens to the information that you provide?}

- The overall findings will form part of a PhD thesis, and/or be published in scientific journals, presented at academic conferences, and/or grant applications.

If you would like to know the results of this study, they will be available approximately November, 2019 through A.Prof Taciano Milfont's research lab website (www.vuwenvironmentallab.wordpress.com).

\section{Thank you for considering participation in this research.}




\section{Appendix 5.C: Study 3 Time 1 survey}

\section{Understanding why people in Wellington prepare for natural hazards}

Thank you for considering taking part in this short survey.

The purpose of this research is to understand why people in Wellington do or do not prepare for natural hazards. You don't need to have done any preparation yourself, just read the following questions carefully and give the answer which best reflects your opinion. Please try to answer all of the questions. In order to complete this survey you must be currently living in Wellington and be 18 years of age or older.

The survey is completely confidential, so your responses cannot be identified. We anticipate that this research will take you about 15 minutes to complete. At the end, you'll have the chance to go into the prize draw for one of three $\$ 100$ supermarket gift cards.

As part of this research, we are also running a follow-up survey one month after we receive the responses to the first survey. This survey will be much shorter (approximately 5 minutes), and is very important to help us understand why people prepare for natural hazards. If you complete this second survey, you will receive another entry into the prize draw.

If you would like to know more about the study before proceeding, please click here.

I have read the information above and hereby give my consent to participate in this research. By participating I confirm that $I$ am over the age of 18 and live in Wellington. I understand that my responses will be kept confidential and that I can withdraw from the study at any point. I understand that some identifying information will be used to collate the data from this research, at which point it will then be destroyed unless I specify otherwise below. All identifying information will either be destroyed or stored securely, as explained in the information sheet and in accordance with the option you choose below. Your contact details (e.g., email address) will ONLY potentially be re-used beyond this study if you select the fourth of the following options. 
Please select one of the following options:

I want my survey responses and all identifying information destroyed as soon as the data has been extracted

My survey responses can be kept for five years but I want all identifying information destroyed immediately after prizes have been allocated

My contact details can be used to send me a short follow-up survey approximately one month after I send back this survey (you will be given the option to enter your email address to receive the second survey), after which I want my identifying information destroyed

My contact details can be kept on a secure mailing list so I can be contacted in future to participate in another similar study, including the one-month follow-up survey

Please select one of the following options:

Yes, I agree to participate

$\bigcirc$
No, I do not want to participate

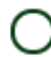

Do you currently live in the Wellington region (including Wellington City, Upper and Lower Hutt, and Porirua)?

Yes

No

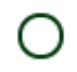

$\bigcirc$

Are you 18 years of age or older?

Yes

No

0

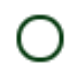


This survey will ask a number of questions about natural hazards. For the purpose of this research, a natural hazard is a process or force of nature, such as an earthquake, flood, tsunami, landslide, wildfire, storm, and volcanic eruption, which has the potential to cause negative consequences such as damage, injury, and/or disruption to lifelines (e.g., roads, water, electricity, food and medical supplies).

Do you understand this definition?

Yes

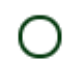

No

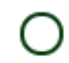

Some of the questions you will see in this survey may seem to be similar, but they do ask for slightly different thinking and are all important. Please read each question and the available responses carefully.

Please answer the following questions by selecting the response which best reflects your agreement with the statement.

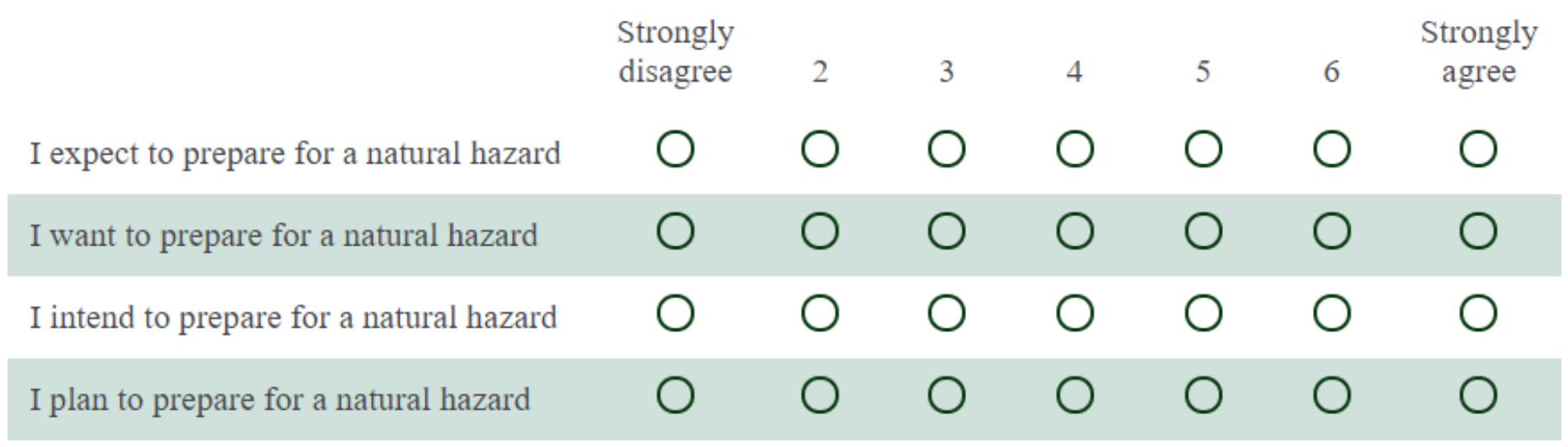


Please answer the following questions by selecting the response which best reflects your opinion about the statement.

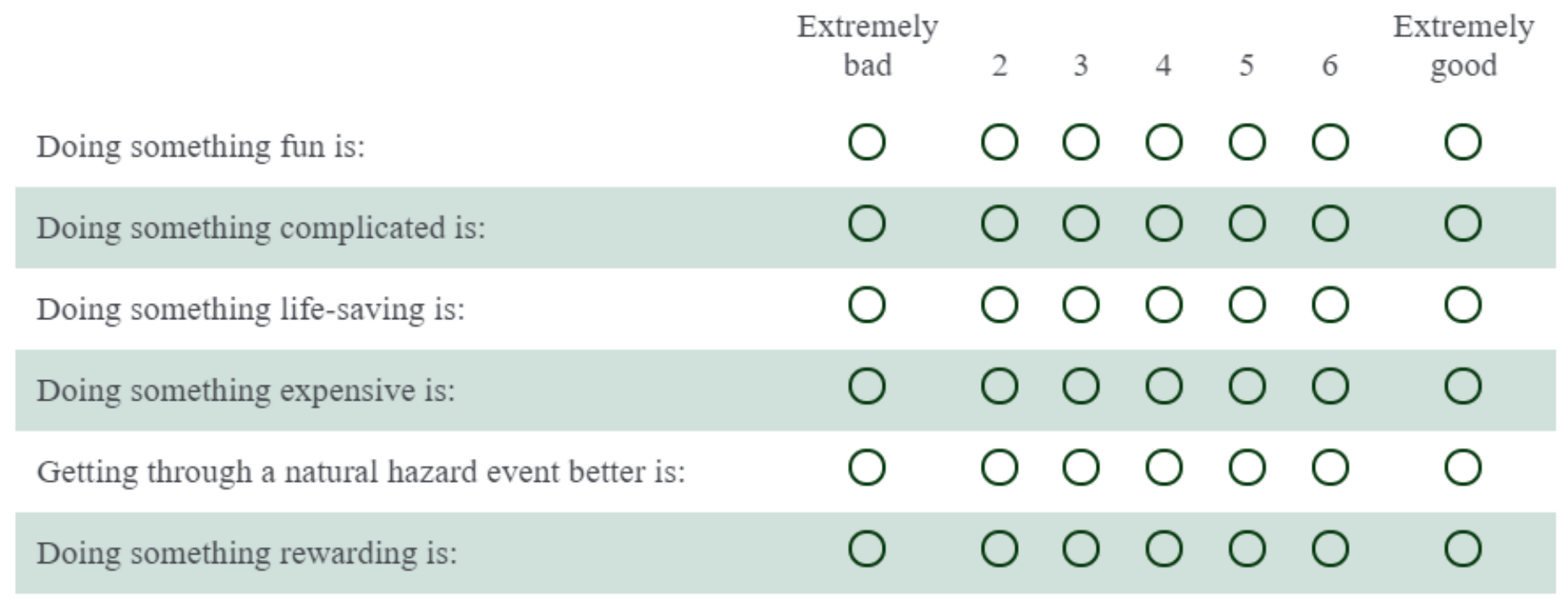

I think that preparing for natural hazards is:

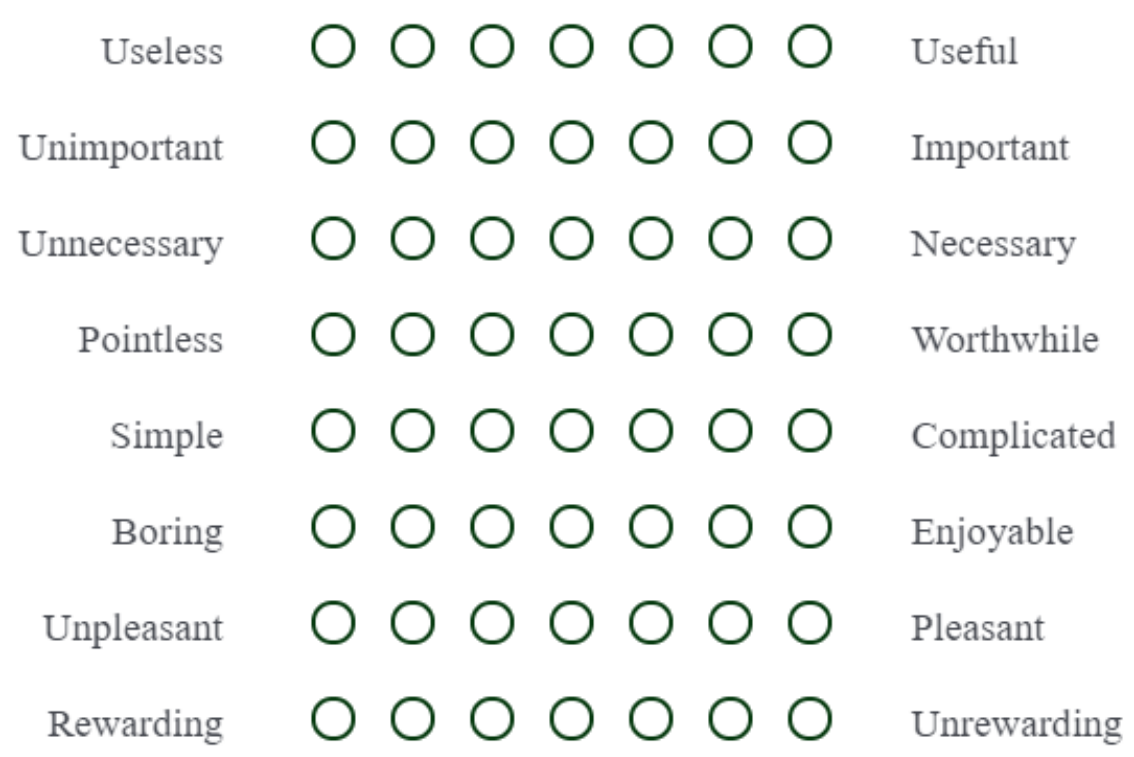


Please answer the following questions by selecting the response which best reflects your agreement with the statement.

$\begin{array}{llllllll}\begin{array}{l}\text { Strongly } \\ \text { disagree }\end{array} & 2 & 3 & 4 & 5 & 6 & \begin{array}{c}\text { Strongly } \\ \text { agree }\end{array}\end{array}$

Preparing for a natural hazard will result in me and my home getting through a hazard event better

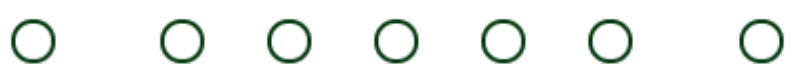

It is complicated to prepare for a natural hazard

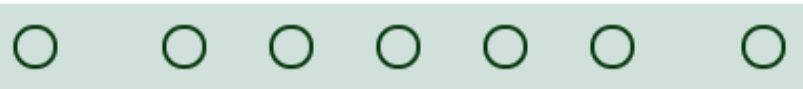

Preparing for a natural hazard can be a rewarding experience

\section{0}

Preparing for a natural hazard can be a fun experience

000000000

It is expensive to prepare for a natural hazard

00000000

I will feel like I'm doing something life-saving if I prepare for a natural hazard

000000 
Among the people who are important to you, how much agreement would there be that preparing for a natural hazard is a good thing to do?

$\begin{array}{ccccccc}\begin{array}{c}\text { No } \\ \text { agreement }\end{array} & 2 & 3 & 4 & 5 & 6 & \begin{array}{c}\text { A great deal of } \\ \text { agreement }\end{array} \\ \bigcirc & 0 & \bigcirc & \bigcirc & 0 & \bigcirc & \bigcirc\end{array}$

Please answer the following questions by selecting the response which best reflects your agreement with the statement. By 'prepared', we mean having done a number of different actions for the event of a natural hazard, not just one or two.

$\begin{array}{llllllll}\begin{array}{l}\text { Strongly } \\ \text { disagree }\end{array} & 2 & 3 & 4 & 5 & 6 & \begin{array}{c}\text { Strongly } \\ \text { agree }\end{array}\end{array}$

Most people like me have prepared for a natural hazard
$\bigcirc$
$\bigcirc$
○
$\bigcirc \bigcirc \bigcirc$
$\mathrm{O}$

Most people who are important to me have prepared for a natural hazard

\section{$0 \quad 0 \quad 0 \quad 0 \quad 0 \quad 0 \quad 0$}

Most people like me approve of my preparing for a natural hazard

0000000

The people in my life whose opinions I value have prepared for a natural hazard

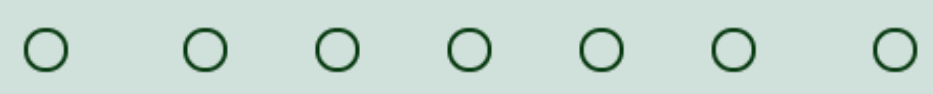

Most people who are important to me approve of my preparing for a natural hazard

$0 \quad 0 \quad 0 \quad 0 \quad 0 \quad 0$

The people in my life whose opinions I value approve of my preparing for a natural hazard

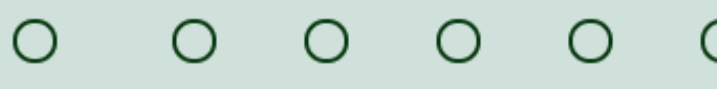

O 0

How many of the people who are important to you do you think have prepared for a natural hazard?

$\begin{array}{ccccccc}\text { None } & 2 & 3 & 4 & 5 & 6 & \text { All } \\ \bigcirc & 0 & 0 & \bigcirc & 0 & \bigcirc & \bigcirc\end{array}$


Please answer the following questions by selecting the response which best reflects your agreement with the statement.

$\begin{array}{lllllll}\begin{array}{l}\text { Strongly } \\ \text { disagree }\end{array} & 2 & 3 & 4 & 5 & 6 & \begin{array}{c}\text { Strongly } \\ \text { agree }\end{array}\end{array}$

When it comes to preparing for a natural hazard, I want to do what my neighbours think I should do

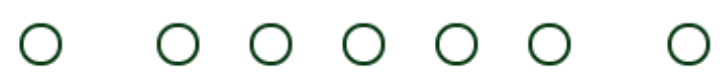

When it comes to preparing for a natural hazard, I want to do what my family and friends think I should do

\section{0}

Wellingtonians approve of my preparing for a natural hazard

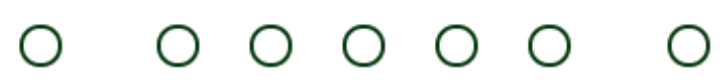

New Zealanders approve of my preparing for a natural hazard

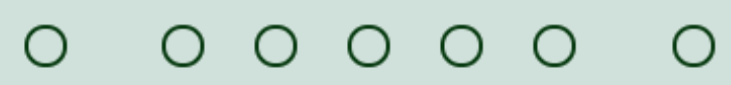

When it comes to preparing for a natural hazard, I want to do what other New Zealanders think I should do

0000000

My family and friends approve of my preparing for a natural hazard

0000000

My neighbours approve of my preparing for a natural hazard

0000000

When it comes to preparing for a natural hazard, I want to do what other Wellingtonians think I should do

0000000 
Please answer the following questions by selecting the response which best reflects your opinion about the statement.

$\begin{array}{ccccccc}\begin{array}{c}\text { Definitely } \\ \text { false }\end{array} & 2 & 3 & 4 & 5 & 6 & \begin{array}{c}\text { Definitely } \\ \text { true }\end{array}\end{array}$

Most New Zealanders have prepared for a natural hazard
$\bigcirc$
$\bigcirc \bigcirc \bigcirc$
$\mathrm{O}$

Most of my neighbours have prepared for a natural hazard

$\circ \bigcirc \bigcirc \bigcirc 00$

O

Most Wellingtonians have prepared for a natural hazard

000000

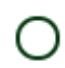

Most of my family and friends have

prepared for a natural hazard

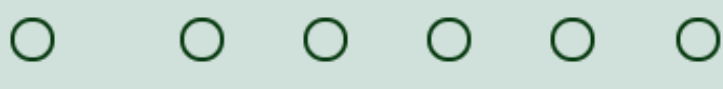

O

Please answer the following questions by selecting the response which best reflects your opinion about the statement.

$\begin{array}{lllllll}\begin{array}{l}\text { Very } \\ \text { much }\end{array} & 2 & 3 & 4 & 5 & 6 & \begin{array}{c}\text { Not at } \\ \text { all }\end{array}\end{array}$

When it comes to preparing for a natural hazard, how much do you want to be like your neighbours?

When it comes to preparing for a natural hazard, how much do you want to be like 000000

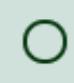
other Wellingtonians?

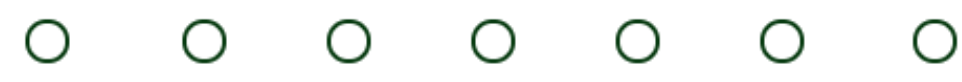

When it comes to preparing for a natural hazard, how much do you want to be like your family and friends?

$0 \quad 0 \quad 0 \quad 0 \quad 0 \quad 0$

When it comes to preparing for a natural hazard, how much do you want to be like other New Zealanders?

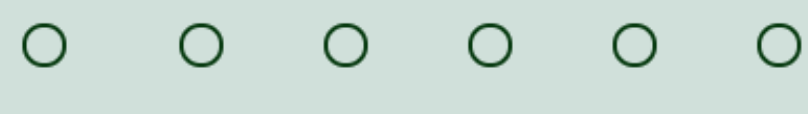


For me to prepare for a natural hazard is...

Extremely easy

O
2

O
3

O
4

O
5

6

O
Extremely difficult

Please answer the following questions by selecting the response which best reflects your agreement with the statement.

$\begin{array}{llllllll}\begin{array}{c}\text { Strongly } \\ \text { disagree }\end{array} & 2 & 3 & 4 & 5 & 6 & \begin{array}{c}\text { Strongly } \\ \text { agree }\end{array}\end{array}$

If I wanted to I could prepare for a natural hazard
$\bigcirc$
$\bigcirc \bigcirc$
0
$\mathrm{O} O$

The decision to prepare for a natural hazard is beyond my control

\section{$\begin{array}{lllllllllll}0 & 0 & 0 & 0 & 0 & 0\end{array}$}

000000

I am confident that I can prepare for a natural hazard

It is mostly up to me whether or not I prepare for a natural hazard
O
O
O

O

O

O

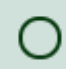

My preparing for a natural hazard is up to me

000000

For me to prepare for a natural hazard is...

Definitely

impossible

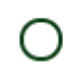

2

$\bigcirc$
3

O
4

0
Definitely possible

0

How much control do you believe you have over preparing for a natural hazard?

$\begin{array}{ccccccc}\begin{array}{c}\text { No } \\ \text { control }\end{array} & 2 & 3 & 4 & 5 & 6 & \begin{array}{c}\text { Complete } \\ \text { control }\end{array} \\ \bigcirc & 0 & 0 & 0 & 0 & 0 & \bigcirc\end{array}$


Please answer the following questions by selecting the response which best reflects your opinion of the likelihood of the statement.

$\begin{array}{cccccccc}\begin{array}{c}\text { Extremely } \\ \text { unlikely }\end{array} & 2 & 3 & 4 & 5 & 6 & \text { likely }\end{array}$

In the next month, I will have the money to prepare for a natural hazard
$\bigcirc$
O
$\bigcirc \bigcirc \bigcirc \bigcirc$
O

In the next month, I will make the effort to prepare for a natural hazard

$\bigcirc \bigcirc \bigcirc \bigcirc \bigcirc$

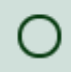

In the next month, I will have the time to

prepare for a natural hazard

000000

In the next month, I will think about

preparing for a natural hazard

$\bigcirc \quad \bigcirc \quad \bigcirc \quad \bigcirc$

$\bigcirc$

Please answer the following questions by selecting the response which best reflects your agreement with the statement.

$\begin{array}{lllllll}\begin{array}{c}\text { Strongly } \\ \text { disagree }\end{array} & 2 & 3 & 4 & 5 & 6 & \begin{array}{c}\text { Strongly } \\ \text { agree }\end{array}\end{array}$

Having the time would make me more likely to prepare for a natural hazard

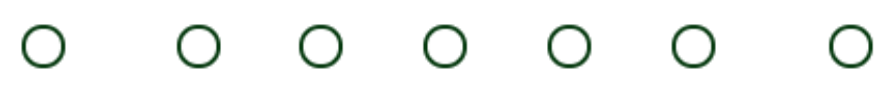

Making the effort to prepare for a natural hazard would make me more likely to prepare
○
00
000
○

Thinking about preparing would make me more likely to prepare for a natural hazard

$\bigcirc 00000$

Having enough money would make me more likely to prepare for a natural hazard
O

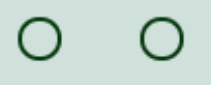
O
$\bigcirc \bigcirc$
O

During the past month, how much preparation have you done for a natural hazard event?
None
2
3
4
5
6
A lot
$\bigcirc$
$\bigcirc$
$\bigcirc$
$\mathrm{O}$
$\mathrm{O}$
○ 
Please indicate which of these preparations you or someone in your household have made for the specific purpose of a natural hazard. If you've done one of these in the past but can no longer benefit from it (e.g., stored food years ago which you've since thrown out) please respond 'No'. If you have done part of the action (e.g., purchased a torch but not a battery-powered radio) please respond 'Partly'.

Not Yes No Partly Unsure applicable

Obtain a fire extinguisher for my household

○ $\quad 0 \quad 0 \quad 0 \quad 0$

Identify people in my neighbourhood who need checking up on in a natural hazard event

$\bigcirc \quad 0 \quad 0$

Store enough emergency supplies to help others not in my household

$\bigcirc \quad \bigcirc \quad 0$

○

Make sure that my house is insured for damage caused by natural hazards

O $0 \quad 0$

O

O

Identify my local Community Emergency Hub so I can volunteer in a natural hazard event

O $\mathrm{O}$

$\mathrm{O}$

$\mathrm{O}$

Have the strength of my building checked (or ask landlord to do the same)

Make an emergency plan (e.g., knowing where to meet family)
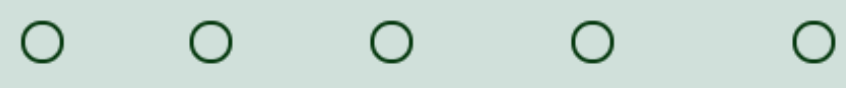

Not
Yes No Nortly applicable

Ensure that heavy objects are stored on the floor and at the bottom of cupboards

$0 \quad 0 \quad 0$

$\mathrm{O}$

Store non-perishable food

O $0 \quad 0$

O

O

Secure movable objects in my home (such as computers and TVs)

O $0 \quad 0 \quad 0 \quad 0$

Seek out information about the different natural hazard risks posed to my home

$0 \quad 0 \quad 0 \quad 0 \quad 0$

Strengthen my house/its foundations (or ask landlord to do the same)

$\begin{array}{lllll}0 & 0 & 0\end{array}$

Cloud-store important documents and/or photos on an internet server

$\begin{array}{lllll}0 & 0 & 0 & 0 & 0 \\ 0 & 0 & 0 & 0 & 0\end{array}$

Store supplies (such as plastic bags and toilet paper) to use as an emergency toilet 


\begin{tabular}{|c|c|c|c|c|c|}
\hline & Yes & No & Partly & Unsure & $\begin{array}{c}\text { Not } \\
\text { applicable }\end{array}$ \\
\hline $\begin{array}{l}\text { Purchase items to use if power is lost such as a } \\
\text { torch, radio, or gas cooker }\end{array}$ & & & & $\cap$ & \\
\hline Fasten tall furniture to the wall & & & & & $\Omega$ \\
\hline Make an emergency kit & 0 & 0 & 0 & 0 & 0 \\
\hline Store water & $\Omega$ & 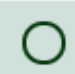 & $\cap$ & $\cap$ & 0 \\
\hline Purchase a water tank & Q & $\Omega$ & 0 & 0 & $\bigcirc$ \\
\hline
\end{tabular}

Are there any other actions you have taken specifically for the purpose of being more prepared for a natural hazard? Please write in the text box below:

Have you or someone close to you suffered damage to your home or possessions in a natural hazard event in the past?

$\begin{array}{ccccccc}\begin{array}{c}\text { No } \\ \text { damage }\end{array} & 2 & 3 & 4 & 5 & 6 & \begin{array}{c}\text { A lot of } \\ \text { damage }\end{array} \\ \bigcirc & \bigcirc & \bigcirc & \bigcirc & 0 & 0 & \bigcirc\end{array}$

Have you or someone close to you been harmed in a natural hazard event in the past?

$\begin{array}{ccccccc}\text { Never } & 2 & 3 & 4 & 5 & 6 & \text { Severely } \\ \bigcirc & \bigcirc & \bigcirc & \bigcirc & \bigcirc & \bigcirc & \bigcirc\end{array}$

Have you experienced natural events which didn't cause you harm or damage but made you feel scared or vulnerable?

$\begin{array}{rrrrrrr}\text { None } & 2 & 3 & 4 & 5 & 6 & \text { Many } \\ \bigcirc & \bigcirc & \bigcirc & \bigcirc & \bigcirc & \bigcirc & \bigcirc\end{array}$


How old are you?

What is your gender?
$\bigcirc$ Male
Female
Other:

With which ethnicity do you most strongly identify?
NZ European/Pākehā
North American
Asian (please specify)
South/Latin American
Pasifika
$\bigcirc$ African (please specify)
Māori
Other (please specify)
$\bigcirc$ European (please specify)

What is the highest level of education you have completed?
Secondary school incomplete
Postgraduate degree
NCEA, School Cert, or other secondary school qualification
Polytechnic or Trade Certificate
Doctorate
$\bigcirc$ Undergraduate degree 


\section{Application ID Number: 000026244}

Thank you for participating in this survey.

This study examines natural hazard preparation in Wellington. It explores the different barriers that might contribute to people not preparing, as well as other related factors such as experience of natural hazards. One goal of this survey is to understand the reasons why people do and do not prepare for natural hazards in Wellington. This will help us to understand what thoughts need to be targeted so that we can increase preparation behavior.

This survey assessed your attitudes towards natural hazard preparation, your perception of social norms (is natural hazard preparation common and desirable in your social groups), as well as whether you think you have control over your own natural hazard preparation. The information we collect in this survey will help us to understand if we should target these factors to purposefully increase natural hazard preparation.

We hope that the information collected in this survey will help us to construct interventions to encourage Wellingtonians to prepare more for natural hazards. Should these interventions be successful, they can then be applied to a wider population through advising and working with relevant organizations such as the EQC, Civil Defence, and local councils.

This research was approved by the School of Psychology Human Ethics Committee under delegated authority of Victoria University of Wellington's Human Ethics Committee (application number: 000026244, convenor: Prof. Paul Jose).

Thank you again for participating in this research.

Lauren Vinnell

PhD Student

School of Psychology

Victoria University of Wellington 
Appendix 5.D: Study 3 Time 2 information sheet

\section{Application ID Number: 000026244}

\author{
Lauren Vinnell \\ PhD Student \\ Email: lauren.vinnell@vuw. \\ ac.nz
}

\author{
A.Prof. Taciano Milfont \\ Primary Supervisor \\ Email: taciano.milfont@vuw. \\ ac.nz
}

Phone: (04) 4636398

\author{
Prof. John McClure \\ Secondary Supervisor \\ Email: john.mcclure@vuw. \\ ac.nz \\ Phone: (04) 4635233 ext \\ 6047
}

\section{What is the purpose of this research?}

- This research will help us to understand why people in Wellington do and do not prepare for natural hazards, including their beliefs about whether it's a common and approved behaviour.

\section{Who is conducting the research?}

-We are a team of researchers in the School of Psychology at Victoria University of Wellington. PhD student Lauren Vinnell is leading this project. Associate Professor Taciano Milfont and Professor John McClure are supervising. This research has been approved by the School of Psychology Human Ethics Committee under delegated authority of Victoria University of Wellington's Human Ethics Committee (application number: 000026244, convenor: Prof. Paul Jose).

\section{What is involved if you agree to participate?}

- If you agree to participate in this study, you will complete a short survey where you will let us know what preparations you've made.

-We anticipate that the survey will take you no more than 5 minutes to complete.

- During the research you are free to withdraw at any point before your survey has been completed.

- As a token of appreciation, you will be given the opportunity to go into the draw for one of three $\$ 100$ supermarket gift cards.

\section{Privacy and Confidentiality}

- This survey is completely confidential.

- Digital copies of your responses will be kept for five years after the publication of this research at which point they will then be destroyed. If in the previous survey you requested that your survey responses be destroyed as soon as the data has been extracted, that request will extend to this survey,

- In accordance with the requirements of some scientific journals and organizations, information from your survey that does not identify you may be shared with other researchers.

- An electronic version of the data used for analyses will remain indefinitely in the custody of the researchers at Victoria University of Wellington. This data will not contain any identifying information.

- At the beginning of the survey, you will be asked to provide your email address. This is so that we can link your responses on this survey to your responses on the previous survey. This is an important part of understanding why people prepare for natural hazards. This identifying information will not be included in the collated dataset. 
What happens to the information that you provide?

- The overall findings will form part of a PhD thesis, and/or be published in scientific journals, presented at academic conferences, and/or grant applications.

If you would like to know the results of this study, they will be available approximately November, 2019 through A.Prof Taciano Milfont's research lab website (www.vuwenvironmentallab.wordpress.com).

Thank you for considering participation in this research. 


\section{Appendix 5.E: Study 3 Time 2 survey}

\section{Understanding why people in Wellington prepare for natural hazards}

Thank you for considering taking part in this short survey, following up on the one you completed approximately a month ago.

The purpose of this research is to understand why people in Wellington do or do not prepare for natural hazards. You don't need to have done any preparation yourself, just read the following questions carefully and give the answer which best reflects your opinion. Please try to answer all of the questions. In order to complete this survey you must be currently living in Wellington and be 18 years of age or older.

The survey is completely confidential, so your responses cannot be identified. We anticipate that this research will take you no more than $\mathbf{5}$ minutes to complete. If you complete this survey, you will receive an entry into the prize draw for one of three $\$ 100$ supermarket gift cards.

If you would like to know more about the study before proceeding, please click here.

I have read the information above and hereby give my consent to participate in this research. By participating I confirm that I am over the age of 18 and live in Wellington. I understand that my responses will be kept confidential and that I can withdraw from the study at any point. I understand that some identifying information will be used to collate the data from this research, at which point it will then be removed from the data set.

Please select one of the following options:

Yes, I agree to participate

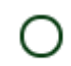

No, I do not want to participate

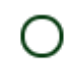


Are you 18 years of age or older?
Yes
No
$\bigcirc$
O

This survey will ask a number of questions about natural hazards. For the purpose of this research, a natural hazard is a process or force of nature, such as an earthquake, flood, tsunami, landslide, wildfire, storm, and volcanic eruption, which has the potential to cause negative consequences such as damage, injury, and/or disruption to lifelines (e.g., roads, water, electricity, food and medical supplies).

In the past month, how much preparation have you done for a natural hazard event?

$\begin{array}{ccccccc}\text { None } & 2 & 3 & 4 & 5 & 6 & \text { A lot } \\ \bigcirc & \bigcirc & \bigcirc & \bigcirc & \bigcirc & \bigcirc & \bigcirc\end{array}$


Please indicate which of these preparations you or someone in your household have made in the past month for the specific purpose of a natural hazard. If you've done one of these in the past but can no longer benefit from it (e.g., stored food years ago which you've since thrown out) please respond 'No'. If you have done part of the action (e.g., purchased a torch but not a battery-powered radio) please respond 'Partly'.

Not

Yes No Partly Unsure applicable
Purchase items to use if power is lost such
as a torch, radio, or gas cooker

Seek out information about the different natural hazard risks posed to my home

O $\quad 0 \quad 0$

$\bigcirc$

Obtain a fire extinguisher for my household $\begin{array}{llllll}0 & 0 & 0 & 0\end{array}$

Store non-perishable food

$\mathrm{O} \mathrm{O}$

$\mathrm{O}$

$\mathrm{O}$

$\mathrm{O}$

Purchase a water tank

$\mathrm{O} \bigcirc$

$\mathrm{O}$

○

○

Fasten tall furniture to the wall

$\mathrm{O} \mathrm{O}$

Have the strength of my building checked

(or ask landlord to do the same)

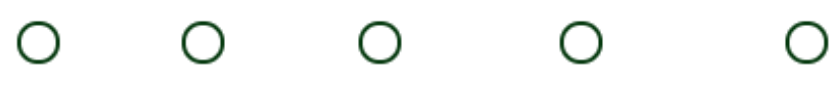

Not

Yes No Partly Unsure applicable

Make sure that my house is insured for damage caused by natural hazards

O $0 \quad 0 \quad 0 \quad 0$

Store supplies (such as plastic bags and toilet paper) to use as an emergency toilet

$0 \quad 0 \quad 0 \quad 0$

Ensure that heavy objects are stored on the floor and at the bottom of cupboards

O $0 \quad 0 \quad 0 \quad 0$

Store enough emergency supplies to help others not in my household

$\bigcirc \quad 0$

$\bigcirc \quad \mathrm{O}$

Cloud-store important documents and/or photos on an internet server

$0 \quad 0 \quad 0 \quad 0 \quad 0$

Identify people in my neighbourhood who need checking up on in a natural hazard event

$\mathrm{O}$

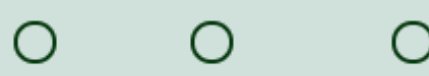

O

Secure movable objects in my home (such as computers and TVs)

$\mathrm{O}$

O

$\mathrm{O}$

$\mathrm{O}$

○ 


\begin{tabular}{|c|c|c|c|c|c|}
\hline & Yes & No & Partly & Unsure & $\begin{array}{c}\text { Not } \\
\text { applicable }\end{array}$ \\
\hline Store water & $\cap$ & $\bigcirc$ & $\bigcirc$ & $\bigcirc$ & 0 \\
\hline $\begin{array}{l}\text { Identify my local Community Emergency } \\
\text { Hub so I can volunteer in a natural hazard } \\
\text { event }\end{array}$ & & & 0 & & \\
\hline $\begin{array}{l}\text { Make an emergency plan (e.g., knowing } \\
\text { where to meet family) }\end{array}$ & 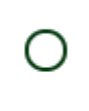 & $\Omega$ & $\Omega$ & $\Omega$ & \\
\hline $\begin{array}{l}\text { Strengthen my house/its foundations (or } \\
\text { ask landlord to do the same) }\end{array}$ & $\Omega$ & 0 & 0 & & \\
\hline Make an emergency kit & $\mathrm{O}$ & 0 & O & O & O \\
\hline
\end{tabular}

Are there any other actions you have taken in the past month specifically for the purpose of being more prepared for a natural hazard? Please write in the text box below:

Would you like to receive an Earthquake Planning Guide? (If you select 'Yes', you'll be provided a link on the next page)

Yes, I'd like an Earthquake Planning Guide

$\mathrm{O}$
No, I don't want an Earthquake Planning Guide

$\mathrm{O}$ 
In the past month, have you or someone close to you suffered damage to your home or possessions in a natural hazard event?

$\begin{array}{ccccccc}\begin{array}{c}\text { No } \\ \text { damage }\end{array} & 2 & 3 & 4 & 5 & 6 & \begin{array}{r}\text { A lot of } \\ \text { damage }\end{array} \\ \bigcirc & 0 & 0 & 0 & 0 & 0 & \bigcirc\end{array}$

In the past month, have you or someone close to you been harmed in a natural hazard event?

$\begin{array}{ccccccc}\text { Never } & 2 & 3 & 4 & 5 & 6 & \text { Severely } \\ \bigcirc & 0 & 0 & 0 & 0 & 0 & \bigcirc\end{array}$

In the past month, have you experienced natural events which didn't cause you harm or damage but made you feel scared or vulnerable?

$\begin{array}{ccccccc}\text { None } & 2 & 3 & 4 & 5 & 6 & \text { Many } \\ & 0 & 0 & 0 & 0 & 0 & \bigcirc\end{array}$




\section{Application ID Number: 000026244}

Thank you for participating in this survey.

This study examines natural hazard preparation in Wellington. It explores the different barriers that might contribute to people not preparing, as well as other related factors such as experience of natural hazards and perceived hazard risk. One goal of this survey is to understand the reasons why people do and do not prepare for natural hazards in Wellington. This will help us to understand what thoughts need to be targeted so that we can increase preparation behavior.

The first survey assessed your attitudes towards natural hazard preparation, your perception of social norms (is natural hazard preparation common and desirable in your social groups), as well as whether you think you have control over your own natural hazard preparation. According to past research, these factors predict behavioural intentions. To test whether people act on their intentions, we need to measure intentions at one time point, and then measure behaviour at a later time. By completing this second survey, you will help us to identify what might be important factors contributing to people's preparation.

We hope that the information collected in this survey will help us to construct interventions to encourage Wellingtonians to prepare more for natural hazards. Should these interventions be successful, they can then be applied to a wider population through advising and working with relevant organizations such as the EQC, Civil Defence, and local councils.

This research was approved by the School of Psychology Human Ethics Committee under delegated authority of Victoria University of Wellington's Human Ethics Committee (application number: 000026244, convenor: Prof. Paul Jose).

Thank you again for participating in this research.

Lauren Vinnell

PhD Student

School of Psychology

Victoria University of Wellington 
On 18 October, 2018, the New Zealand ShakeOut earthquake drill was held nationwide (www.shakeout.govt.nz). We are doing a post-ShakeOut follow-up survey and would like to hear about your experiences of it, so we've set up this online questionnaire that we'd like you to fill out for us. It won't take long - only about 15 minutes of your time. If you haven't heard of, or didn't participate in ShakeOut we would still be interested in your perspectives in this survey.

The findings from this survey will be used to help communities better understand and prepare for future earthquakes.

All replies will be anonymous, and we will only report on general trends. You are not asked to record your name. Filling in the survey implies that you are consenting to participate. You should be 18 years or over to participate.

We want to thank you in advance for your cooperation. Your views are very important to the success of this study, and we look forward to your response.

Regards,

Lauren

Vinnell

Victoria University of Wellington

Julia

Becker

Joint Centre for Disaster Research (Massey University, Wellington)

For further information, please contact Lauren Vinnell at lauren.vinnell@vuw.ac.nz

This project has been evaluated by peer review through Massey University, and judged to be low risk. Consequently, it has not been reviewed by one of the University's Human Ethics Committees. The researchers named above are responsible for the ethical conduct of this research.

If you have any concerns about the conduct of this research that you wish to raise with someone other than the researchers, please contact Professor Craig Johnson, Director (Research Ethics), telephone (06) 3569099 ext. 85271, or email humanethics@massey.ac.nz 
Which region of New Zealand did you live in during the New Zealand ShakeOut drill on 18 October 2018?

What should you do when you feel an earthquake and you are inside a building? (Please state briefly)

What should you do if you feel an earthquake and you are outside? (Please state briefly)

Did you know about the New Zealand ShakeOut drill when it was held on 18 October $2018 ?$

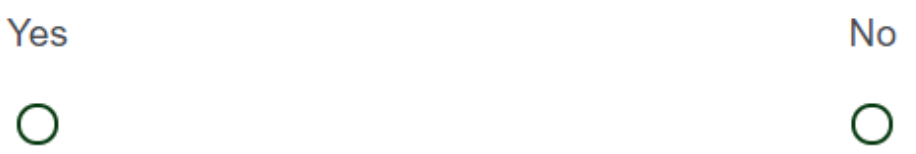

Did you participate in the New Zealand ShakeOut earthquake drill held on 18 October 2018? (Note: this could include two weeks either side of the drill date)

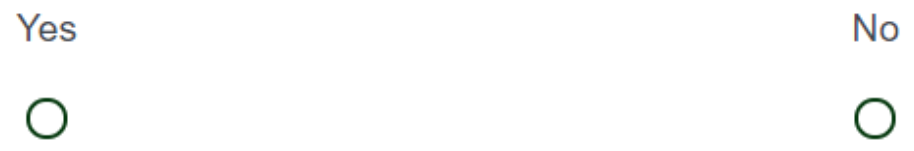


Did you drop, cover and hold for New Zealand ShakeOut 2018?

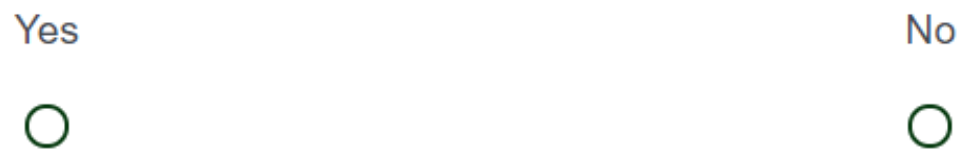

If you did not drop, cover and hold, why not? (Tick all that apply)

$\square$ Disability

Age (fragility)

Embarrassment $\square$ Did not have a place to drop, cover and hold (no table or desk available)

$\square$ Did not know to do this

Acting as observer $\square$ Intended to, but forgot

$\square$ Other (please specify)

Did you undertake any of the following activities as part of the New Zealand ShakeOut 2018 ? (Tick all that apply)

$\square$ Participated in a relevant earthquake-related meeting(s)

$\square$ Got preparedness items organised at home

Got preparedness items organised at work

$\square$ Got preparedness items organised as a community

Helped others prepare $\square$ Made an emergency plan at home (e.g., how to respond after an earthquake)

$\square$ Made an emergency plan at work (e.g., how to respond after an earthquake)

$\square$ Made an emergency plan for your community (e.g., how to respond after an earthquake)

$\square$ Carried out a tsunami practice evacuation walk (hīkoi) $\square$ Did an exercise in conjunction with the drill

$\square$ Didn't do any of the actions listed

Had already done all of the actions listed

$\square$ Other (please specify)

Do you think about how you would evacuate for a tsunami when you are not at home/work (e.g., on holiday)?

$\begin{array}{ccccc}\text { Never } & \text { Sometimes } & \text { About half the time } & \text { Most of the time } & \text { Always } \\ & 0 & O & O & \bigcirc\end{array}$


Please answer the following questions even if you hadn't heard of, or did not participate in, New Zealand ShakeOut 2018

Have you sought any information about earthquakes or preparing for emergencies since New Zealand ShakeOut 2018 (or from the date of 18 October 2018, if you had not heard of/participated in ShakeOut)?

Yes

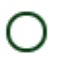

No

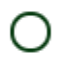

What was the information you sought about? (Tick all that apply)

$\square$ The 'science' of earthquakes

$\square$ How to specifically prepare or plan for earthquakes

$\square$ What might happen in an earthquake (i.e. the impacts of an earthquake)

$\square$ How to prepare or plan for emergencies in general

Where did you get that information from? (Tick all that apply)

\begin{tabular}{|c|c|c|c|}
\hline $\begin{array}{l}\square \text { Emergency services } \\
\text { (e.g., police, fire, } \\
\text { ambulance) }\end{array}$ & $\begin{array}{l}\square \text { GeoNet website } \\
\text { (www.geonet.org.nz) }\end{array}$ & $\begin{array}{l}\square \text { Ministry of Civil } \\
\text { Defence \& Emergency } \\
\text { Management }\end{array}$ & $\begin{array}{l}\square \text { Social media } \\
\text { (e.g., } \\
\text { Facebook, } \\
\text { Twitter) }\end{array}$ \\
\hline $\begin{array}{l}\text { EQC (including the } \\
\text { EQC website: } \\
\text { www.eqc.govt.nz) }\end{array}$ & $\square$ Insurance companies & $\begin{array}{l}\square \text { New Zealand ShakeOut } \\
\text { website } \\
\text { (www.shakeout.govt.nz) }\end{array}$ & $\begin{array}{l}\square \text { Internet (e.g., } \\
\text { Google } \\
\text { search, } \\
\text { YouTube) }\end{array}$ \\
\hline$\square$ Family & $\begin{array}{l}\square \text { Local community } \\
\text { members (e.g., } \\
\text { friends, neighbours) }\end{array}$ & $\square$ Child's school & $\square$ Workplace \\
\hline $\begin{array}{l}\square \text { Get Ready Get Thru } \\
\text { website } \\
\text { (www.getthru.govt.nz) }\end{array}$ & $\begin{array}{l}\square \text { Local council (e.g., } \\
\text { city, district, regional } \\
\text { council) }\end{array}$ & $\begin{array}{l}\square \text { Media (e.g., TV, radio, } \\
\text { newspaper) }\end{array}$ & $\begin{array}{l}\square \text { Other (please } \\
\text { specify) }\end{array}$ \\
\hline$\square$ GNS Science & $\begin{array}{l}\square \text { Local Civil Defence } \\
\text { Emergency } \\
\text { Management }\end{array}$ & & \\
\hline
\end{tabular}


Have you participated in any earthquake drills since New Zealand ShakeOut 2018 was held?

$\begin{array}{ll}\text { Yes } & \text { No } \\ \bigcirc & \bigcirc\end{array}$

Have you practised drop, cover and hold during an earthquake drill since New Zealand ShakeOut 2018?

$\begin{array}{ll}\text { Yes } & \text { No } \\ \bigcirc & \bigcirc\end{array}$

Have you undertaken the actions drop, cover and hold during a real earthquake since New Zealand ShakeOut 2018?

Yes, I did drop, cover and hold when an earthquake happened

No, I did not drop, cover and hold when an earthquake happened

Partly, I did drop, cover and hold for some earthquakes but not others

I have not felt any earthquakes since 18 October 2018

Since New Zealand ShakeOut 2018 (or October 2018):

Once a
week or
more

I think about earthquake issues and problems in my

community

I talk about earthquake problems and issues with others in my community

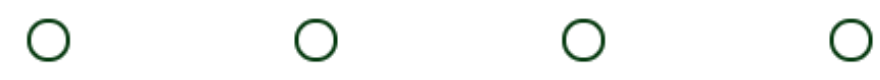

A few times

Once a

month Rarely Never 
Please describe the extent to which you agree or disagree with each of the following statements: (Tick one option on each line)

\section{Strongly agree}

Earthquakes are too destructive to bother preparing for
Neither

agree nor disagree
Strongly

Disagree disagree

\section{Earthquakes are going}

to cause widespread loss of life and property

O

$\mathrm{O} \quad \mathrm{O}$

$\bigcirc$

$\mathrm{O}$ whether we prepare for

them or not

If people make

preparations for

earthquakes they are

$\mathrm{O}$

$\bigcirc$

$\bigcirc$

O

$\bigcirc$ almost certain not to

work

There is nothing people

can do about

earthquakes, so there is

no point trying to

$\mathrm{O} \quad \mathrm{O}$

O $\mathrm{O}$

0

$\bigcirc$

prepare for that

emergency

The way I look at it,

nothing is going to help

if there was an

○ $\quad 0$

O $\quad 0$

○

$\bigcirc$

earthquake

How prepared do you think you are for a major earthquake?

$\begin{array}{ccccccc}\text { Not prepared } & 2 & 3 & \begin{array}{c}\text { Moderately } \\ \text { prepared }\end{array} & 5 & 6 & \begin{array}{r}\text { Very well } \\ \text { prepared }\end{array} \\ \bigcirc & 0 & 0 & 0 & 0 & 0 & \bigcirc\end{array}$

Think of an acquaintance (someone you know only slightly) who lives in New Zealand. How prepared do you think they are for a major earthquake?

$\begin{array}{ccccccr}\text { Not prepared } & 2 & 3 & \begin{array}{c}\text { Moderately } \\ \text { prepared }\end{array} & 5 & 6 & \begin{array}{r}\text { Very well } \\ \text { prepared }\end{array} \\ \bigcirc & 0 & 0 & \bigcirc & 0 & 0 & \bigcirc\end{array}$


If a major earthquake were to occur in your region, how likely do you think it is that it would cause:

\begin{tabular}{|c|c|c|c|c|c|c|c|}
\hline & $\begin{array}{l}\text { Very } \\
\text { unlikely }\end{array}$ & $\begin{array}{c}\text { Moderately } \\
\text { unlikely }\end{array}$ & $\begin{array}{l}\text { Slightly } \\
\text { unlikely }\end{array}$ & $\begin{array}{l}\text { Neither } \\
\text { likely } \\
\text { nor } \\
\text { unlikely }\end{array}$ & $\begin{array}{l}\text { Slightly } \\
\text { likely }\end{array}$ & $\begin{array}{l}\text { Moderately } \\
\text { likely }\end{array}$ & $\begin{array}{l}\text { Very } \\
\text { likely }\end{array}$ \\
\hline harm to you & $\bigcirc$ & $\bigcirc$ & $\bigcirc$ & $\bigcirc$ & $\mathrm{O}$ & O & $\mathrm{O}$ \\
\hline damage to your property & $\bigcirc$ & $\bigcirc$ & $\bigcirc$ & O & O & $\bigcirc$ & $\mathrm{O}$ \\
\hline $\begin{array}{l}\text { harm to the acquaintance } \\
\text { you thought of when } \\
\text { answering the previous } \\
\text { question }\end{array}$ & O & $\bigcirc$ & $\mathrm{O}$ & 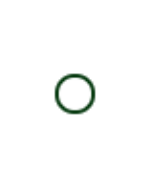 & $\mathrm{O}$ & $\bigcirc$ & 0 \\
\hline $\begin{array}{l}\text { damage to the property of } \\
\text { the acquaintance you } \\
\text { thought of }\end{array}$ & $\mathrm{O}$ & $\mathrm{O}$ & $\mathrm{O}$ & $\bigcirc$ & $\mathrm{O}$ & $\bigcirc$ & $\mathrm{O}$ \\
\hline
\end{tabular}

Some of the questions you will see in this section may seem to be similar, but they do ask for slightly different thinking and are all important. Please read each question and the available responses carefully.

Please answer the following questions by selecting the response which best reflects your agreement with the statement.

\begin{tabular}{|c|c|c|c|c|c|c|}
\hline & $\begin{array}{l}\text { Strongly } \\
\text { disagree }\end{array}$ & 2 & 3 & 4 & 5 & $\begin{array}{c}\text { Strongly } \\
\text { agree }\end{array}$ \\
\hline I intend to prepare for earthquakes & $\bigcirc$ & 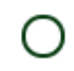 & & & & C \\
\hline I expect to prepare for earthquakes & & & & & & \\
\hline I plan to prepare for earthquakes & 0 & $\bigcirc$ & $\cap$ & $\frown$ & & $\bigcirc$ \\
\hline I want to prepare for earthquakes & $\cap$ & $\Omega$ & $\frown$ & $\cap$ & & $\mathrm{O}$ \\
\hline
\end{tabular}


I think that preparing for earthquakes is:

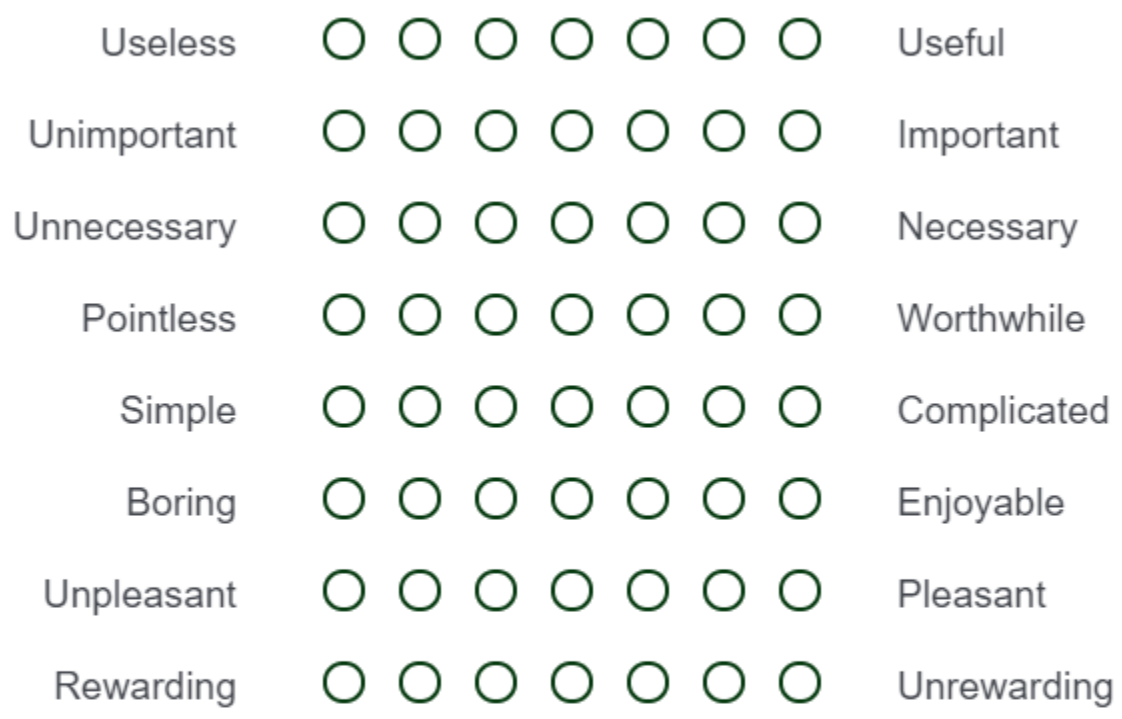

Please answer the following questions by selecting the response which best reflects your agreement with the statement. By 'prepared', we mean having done a number of different actions for the event of an earthquake, not just one or two.

$\begin{array}{llllllll}\begin{array}{c}\text { Strongly } \\ \text { disagree }\end{array} & 2 & 3 & 4 & 5 & 6 & \begin{array}{c}\text { Strongly } \\ \text { agree }\end{array}\end{array}$

The people in my life whose opinions I value approve of my preparing for
O
$\bigcirc$
$\bigcirc$
$\bigcirc \bigcirc$
$\bigcirc \bigcirc$ earthquakes

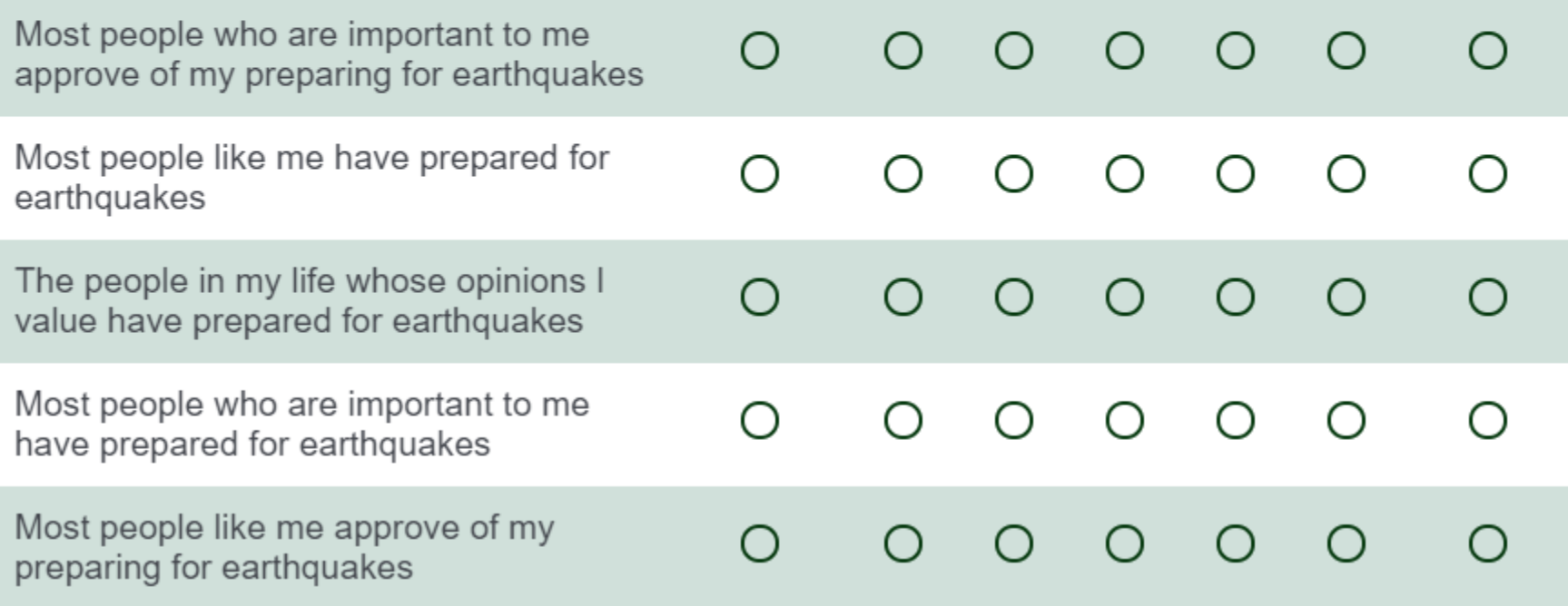


Among the people who are important to you, how much agreement would there be that preparing for earthquakes is a good thing to do?

$\begin{array}{ccccccc}\begin{array}{c}\text { No } \\ \text { agreement }\end{array} & 2 & 3 & 4 & 5 & 6 & \begin{array}{c}\text { A great deal } \\ \text { of agreement }\end{array} \\ \bigcirc & \bigcirc & \bigcirc & \bigcirc & \bigcirc & \bigcirc & \bigcirc\end{array}$

How many of the people who are important to you do you think have prepared for earthquakes?

$\begin{array}{ccccccc}\text { None } & 2 & 3 & 4 & 5 & 6 & \text { All } \\ \bigcirc & \bigcirc & \bigcirc & \bigcirc & \bigcirc & \bigcirc & \bigcirc\end{array}$

Please answer the following questions by selecting the response which best reflects your agreement with the statement.

\begin{tabular}{|c|c|c|c|c|c|c|c|}
\hline & $\begin{array}{l}\text { Strongly } \\
\text { disagree }\end{array}$ & 2 & 3 & 4 & 5 & 6 & $\begin{array}{l}\text { Strongly } \\
\text { agree }\end{array}$ \\
\hline $\begin{array}{l}\text { If I wanted to I could prepare for } \\
\text { earthquakes }\end{array}$ & 0 & O & ○ & $\bigcirc$ & 0 & 0 & 0 \\
\hline $\begin{array}{l}\text { I am confident that I can prepare for } \\
\text { earthquakes }\end{array}$ & 0 & $\bigcirc$ & $\bigcirc$ & 0 & 0 & 0 & 0 \\
\hline $\begin{array}{l}\text { My preparing for earthquakes is up to } \\
\text { me }\end{array}$ & 0 & 0 & 0 & 0 & 0 & 0 & 0 \\
\hline $\begin{array}{l}\text { It is mostly up to me whether or not I } \\
\text { prepare for earthquakes }\end{array}$ & 0 & 0 & 0 & 0 & 0 & 0 & 0 \\
\hline $\begin{array}{l}\text { The decision to prepare for } \\
\text { earthquakes is beyond my control }\end{array}$ & $\bigcirc$ & $\bigcirc$ & $\bigcirc$ & O & $\mathrm{O}$ & $\mathrm{O}$ & O \\
\hline
\end{tabular}


Appendices

For me to prepare for earthquakes is...

$\begin{array}{ccccccc}\begin{array}{c}\text { Extremely } \\ \text { easy }\end{array} & 2 & 3 & 4 & 5 & 6 & \begin{array}{c}\text { Extremely } \\ \text { difficult }\end{array} \\ \bigcirc & \bigcirc & \bigcirc & \bigcirc & \bigcirc & \bigcirc & \bigcirc\end{array}$

For me to prepare for earthquakes is...

$\begin{array}{ccccccr}\begin{array}{c}\text { Definitely } \\ \text { impossible }\end{array} & 2 & 3 & 4 & 5 & 6 & \begin{array}{r}\text { Definitely } \\ \text { possible }\end{array} \\ \bigcirc & \bigcirc & \bigcirc & \bigcirc & \bigcirc & 0 & \bigcirc\end{array}$

How much control do you believe you have over preparing for earthquakes?

$\begin{array}{ccccccc}\begin{array}{c}\text { No } \\ \text { control }\end{array} & 2 & 3 & 4 & 5 & 6 & \begin{array}{r}\text { Complete } \\ \text { control }\end{array} \\ \bigcirc & 0 & 0 & 0 & 0 & 0 & \bigcirc\end{array}$


The following are things that can be done to minimise damage and disruption if an earthquake occurs. In regard to your household, please record whether you: (Tick one option on each line)

$\begin{array}{cccc}\begin{array}{c}\text { Had done } \\ \text { this before } \\ \text { New }\end{array} & \begin{array}{c}\text { Have done } \\ \text { this for or } \\ \text { since New }\end{array} & & \\ \begin{array}{c}\text { Zealand } \\ \text { ShakeOut }\end{array} & \begin{array}{c}\text { Zealand } \\ \text { ShakeOut }\end{array} & \text { Intend to do } & \text { Will not do } \\ 2018 & 2018 & \text { this } & \text { this }\end{array}$

$\mathrm{N} / \mathrm{A}$

Considered the risk of a major earthquake when deciding to live in the house that I live
O
O
O

O

O in now

\section{Fastened tall furniture} to the wall
O
$\mathrm{O}$

○

O

Fastened my hot water cylinder

$\bigcirc \quad \bigcirc$
O

$\mathrm{O}$

\section{Either strengthened my chimney, or satisfied myself that it will probably not fall down in a major earthquake}

\section{Either strengthened} my house to increase its earthquake resistance, or satisfied myself that it will probably not fall down in a major earthquake

\section{Ensured that my roof} will probably not collapse in a major earthquake

\section{Arranged the} cupboards so that heavy objects are stored at ground level

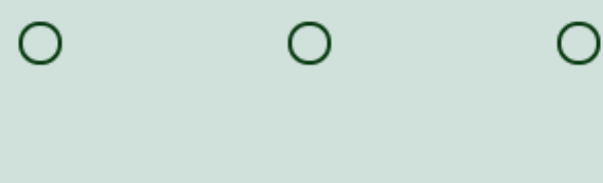

O

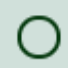

O

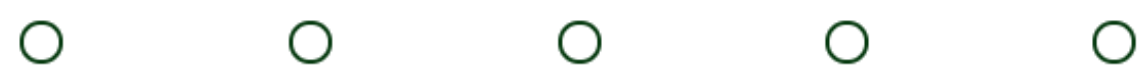


Securely fastened cupboards with

$\bigcirc \quad \bigcirc$

O

O

O latches

Ensured that objects that contain water have not been stored on top of electrical equipment (e.g. a pot

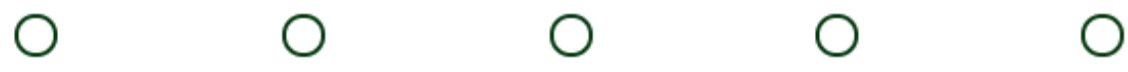
plant or fishbowl on top of the stereo/speakers)

\section{Ensured that heavy} objects are stored on

○

O

O

0 the floor

Put aside spare plastic bags and toilet paper for use as an

$\bigcirc \quad \bigcirc$

O

O emergency toilet

\section{Accumulated enough tools to make minor repairs to the house following an earthquake}

Purchased or put together a first aid kit

Checked if your neighbour is going to need help in an emergency

Made sure that my house is insured for damage caused by earthquakes

\section{Inventoried my} household contents for the purpose of making a claim following a damaging earthquake
O $\quad 0 \quad 0 \quad 0 \quad 0$

O

O

○

O
(1) 
The following are things that can be done to minimise damage and disruption if an earthquake occurs. In regard to your household, please record whether you: (Tick one option on each line)

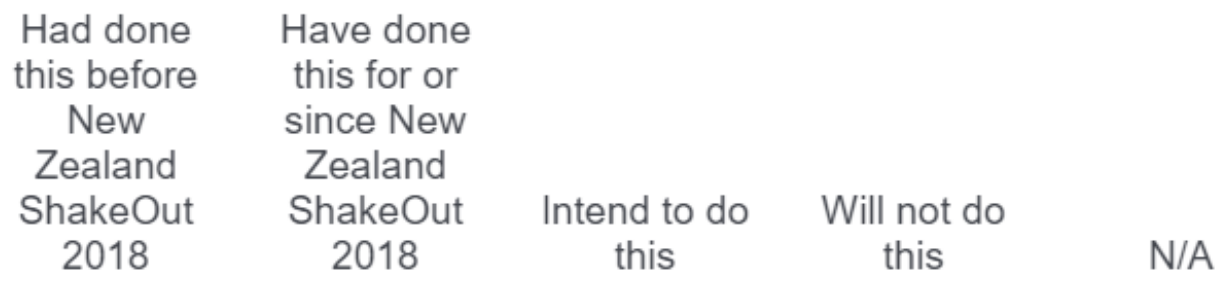

Made a supply of essential medicines for illness or allergies
O
○
O
O
$\mathrm{O}$

Obtained a working battery radio (or solar/dynamo

O $0 \quad 0$

$\bigcirc$

O

equivalent)

Obtained a working battery torch (or solar/dynamo

O $0 \quad 0$

$0 \quad 0$

$\bigcirc$ equivalent)

\section{Secured moveable objects in my home (e.g., TV, computer)}

O $\quad 0$

○

O

○

Made sure that you have access to an alternative cooking source (e.g., gas barbecue)

\section{Made a household disaster/earthquake emergency plan}

Made a household plan that covers where the family should meet if an earthquake occurs during the day

Obtained a working fire extinguisher

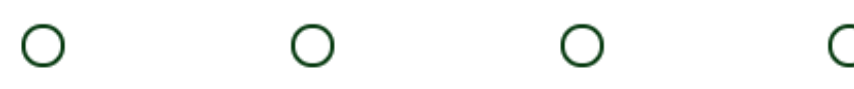

$\bigcirc \quad \bigcirc$
O

0

$\bigcirc$
0
0
0
O
$\bigcirc$
○ 
Obtained a working

fire extinguisher

Obtained spare

batteries for the appliances I might

need to use

Specifically put

together a 'get away'

kit

Checked the contents/operation of my emergency supplies at least every six months

Stored at least 3 litres of water (in plastic containers) per person, per day for 7 days $\bigcirc \quad 0$

$\bigcirc \quad \bigcirc \quad \bigcirc$
O

O

O

$\bigcirc$

$\mathrm{O}$

Stored at least 7 days supply of dehydrated

$\bigcirc \quad 0 \quad 0$ or canned food

O $\quad 0 \quad 0$

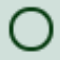

\section{Taken some}

preparedness actions

O

O

O

0

O

at work

Carried out a practice tsunami evacuation

O

O

0

O

walk (hīkoi) 
If you feel you have increased your preparedness since October 2018, please describe the extent to which you agree or disagree with each of the following statements: (Tick one option on each line)

$\begin{array}{lccccc} & \begin{array}{c}\text { Strongly } \\ \text { agree }\end{array} & \text { Agree } & \begin{array}{c}\text { Neither } \\ \text { agree nor } \\ \text { disagree }\end{array} & \text { Disagree } & \begin{array}{c}\text { Strongly } \\ \text { disagree }\end{array} \\ \begin{array}{l}\text { NZ ShakeOut influenced my } \\ \text { increase in preparedness }\end{array} & 0 & 0 & 0 & 0 & \bigcirc \\ \begin{array}{l}\text { An earthquake I experienced } \\ \text { influenced my increase in } \\ \text { preparedness }\end{array} & 0 & 0 & 0 & 0 & \bigcirc\end{array}$

An earthquake that someone

I know was involved in influenced my increase in

$\bigcirc$

$\bigcirc$

$\bigcirc$

$\bigcirc$ preparedness

An earthquake that I learned about through the media influenced my increase in

$\bigcirc$

$\bigcirc$

O

O preparedness

Another event or factor influenced my increase in preparedness (please

$\bigcirc$ specify) 
How old are you?

What is your gender?
Male
Female
Gender diverse

With which ethnicity do you most strongly identify?
O NZ European/Pākehā
North American
Asian (please specify)
South/Latin American
Pasifika
$\bigcirc$ African (please specify)
Māori
Other (please specify)
European (please specify)

What is the highest level of education you have completed?
Secondary school incomplete
Postgraduate degree
$\bigcirc$ NCEA, School Cert, or other secondary school qualification
Masters degree
Polytechnic or Trade Certificate
Doctorate
$\bigcirc$ Undergraduate degree

Thank you for completing this survey. By understanding the role of the New Zealand ShakeOut drill in assisting with response and preparedness for earthquakes, more effective earthquake education can be created. 